Characterization and Reactivity of Organic Monolayers on Gold and Platinum Surfaces

by

Wu, Chien-Ching

PHD Thesis submitted to Iowa State University

Ames Laboratory, U.S. DOE

Iowa State University

Ames, Iowa 50011

Date Transmitted: December 6, 1995

PREPARED FOR THE U.S. DEPARTMENT OF ENERGY

UNDER CONTRACT NO. W-7405-Eng-82. 


\section{DISCLAIMER}

This report was prepared as an account of work sponsored by an agency of the United States Government. Neither the United States Government nor any agency thereof, nor any of their employees, makes any warranty, express or implied, or assumes any legal liability or responsibility for the accuracy, completeness or usefulness of any information, apparatus, product, or process disclosed, or represents that its use would not infringe privately owned rights. Reference herein to any specific commercial product, process, or service by trade name, trademark, manufacturer, or otherwise, does not necessarily constitute or imply its endorsement, recommendation, or favoring by the United States Government or any agency thereof. The views and opinions of authors expressed herein do not necessarily state or reflect those of the United States Government or any agency thereof. 


\title{
Characterization and reactivity of organic monolayers on gold and platinum surfaces
}

\section{Chien-Ching Wu}

\author{
Major Professor: Dr. Marc D. Porter \\ Iowa State University
}

The focus of this study is to understand how the immobilization, dielectric, orientation, composition, coverage, and structure of selfassembled organic monolayers on metal surfaces affects the surface reactivities and properties of these films in order to facilitate the construction of desired films.

Two model systems used: tiols at gold and aromatic acids at platinum. Both methyl- and carboxylic acid-terminated thiolate monolayers were formed at evaporated gold films by the self-assembly technique via the thiol moiety of thiols. The phenylcarboxylic acid and pyridylcarboxylic acid monolayers were formed at platinum electrodes cleaned electrochemically in $1 \mathrm{~N} \mathrm{H}_{2} \mathrm{SO}_{4}$ aqueous solution.

Several surface analysis technologies (including contact angle measurement, electrochemistry, ellipsometry, infrared reflection absorption spectroscopy (IRRAS), and x-ray photospectroscopy) have been used to study the formation, composition, structure, surface properties and reactivity of self-assembled organic monolayers on gold and platinum surfaces.

IRRAS, contact angle measurements, and an electrochemistry methods were used to determine the surface $\mathrm{pK}_{\mathrm{a}}$ of phenylcarboxylic acids and pyridylcarboxylic acids monolayers on platinum.

These techniques were also used to determine the orientation of polymethylene chain axis and the carboxylic follow the structural evolution of the chains and end group of the thiolate monolayers during formation. 
In addition, IRRAS was used to asses the carboxylic acid group in terms of its possible existence as the non-hydrogen-bonded species, the hydrogen-bonded dimeric group, and the hydrogen-bonded polymeric group. The existence of these different forms of the end group were also followed as a function of coverage and as well as their influence a reactivity as a function of solution $\mathrm{pH}$.

IRRAS and contact angle measurements were used to calculate the rate constant of the esterification of carboxylic acid-terminated monolayers on gold. 


\section{DEDICATION}

This thesis is dedicated to the love from my mother Wei Liu and my father Yu-Wey Wu who passed away on December 24, 1993. 


\section{TABLE OF CONTENTS}

LIST OF TABLES.......................................................................... viii

GENERAL INTRODUCTION................................................... 1

Dissertation Organization...................................................... 1

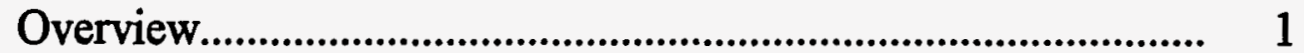

PAPER I. ELECTRON TRANSFER OF

FERRICYANIDE AT ORGANIC

CARBOXYLIC ACID ON

PLATINUM SURFACES ....................... 3

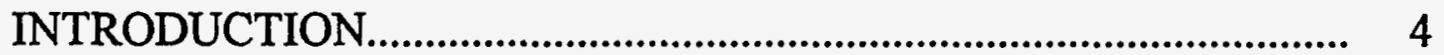

EXPERIMENTAL SECTION..................................................... 6

Electrode preparation.......................................................... 6

Monolayer preparation...................................................... 6

Instrumentation and measurements................................... 7

Electrochemistry.................................................. 7

Contact angles....................................................... 7

Infrared spectroscopy........................................... 8

Chemicals and reagents..................................................... 8

RESULTS AND DISCUSSION......................................................... 9

Characterization of monolayers.......................................... 9

Electrochemical characterization of acid-base reactivity... 14

Potential change at the reaction plane................................ 21

Determination of $\mathrm{pK}_{\mathrm{a}}$ using electrochemistry method....... 28

Determination of $\mathrm{pK}_{\mathrm{a}}$ using IRRAS.................................. 28

Determination of $\mathrm{pK}_{\mathrm{a}}$ using contact angle measurements.. $\quad 31$

Comparison of $\mathrm{pK}_{\mathrm{a}}$ values................................................ 33

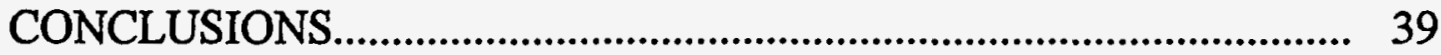

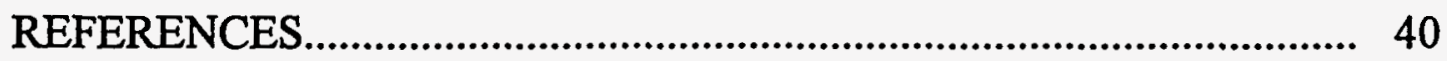




\section{PAPER II. INFRARED REFLECTION ABSORPTION SPECTROSCOPIC STUDY ON THE TEMPORAL EVOLUTION OF THE STRUCTURE OF ALKANE THIOLATE MONOLAYERS ON GOLD.}

INTRODUCTION 44

EXPERIMENTAL SECTION. 47

Substrate preparation. 47

Monolayer preparation 47

Ellipsometric measurements of film thickness

Infrared spectroscopy 47

Chemicals and reagents..................................................... 48

RESULTS AND DISCUSSION........................................................... 49

IRRAS spectra as a function of immersion time.................. 49

MHA/Au............................................................. 49

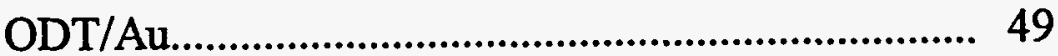

Agostic Bonds..................................................................... 57

ODT on Au covered by a sulfur monolayer........................ 57

Evidence for agostic bonds................................................. 60

The evolution of the structure of MHA/Au and ODT/Au... 61

The polymethylene chain.......................................... 61

The carbonyl stretching mode.................................. 62

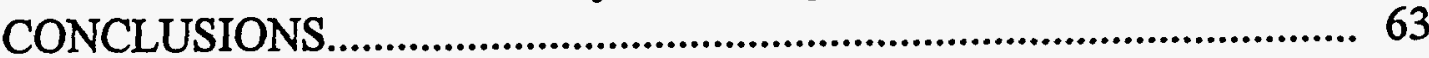

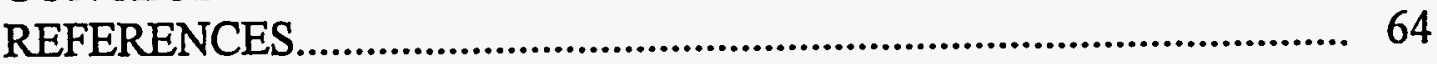

PAPER III. COVERAGE DEPENDENCE OF

CARBONYL STRUCTURE OF 16MERCAPTOHEXADECANOIC ACID MONOLAYERS AT GOLD

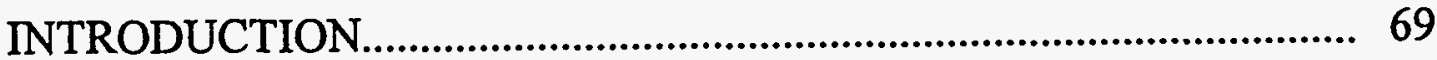

EXPERIMENTAL SECTION............................................................... 71

Substrate preparation......................................................... 71

Monolayer preparation.......................................................... 71 


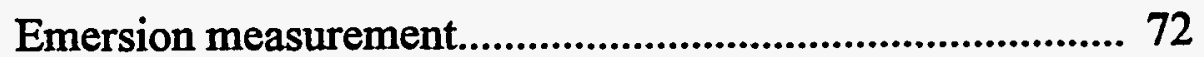

Ellipsometric measurements of film thickness..................... 72

Contact angle measurements................................................ 72

Infrared spectroscopy............................................................ 73

Chemicals and reagents.................................................... 74

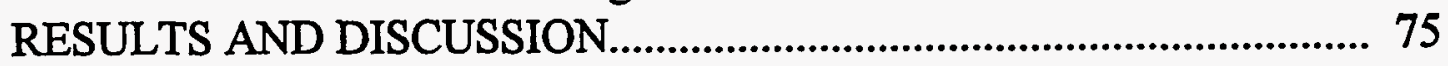

General observations of monolayer formation..................... 75

Ellipsometric thickness as a function of immersion

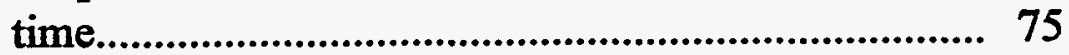

Wetting as a function of immersion time................. 75

IRRAS spectra as a function of immersion time....... 79

IRRAS characterization.................................................. 83

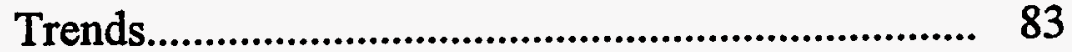

Spectral deconvolution............................................ 87

Methylene stretching region.......................... 87

Carbonyl stretching region.............................. 93

The average tilt angle of carbonyl....... 93

Three carbonyl modes......................... 95

The hydrogen-bonded polymeric $\mathrm{C}=\mathrm{O}$ mode.................................................... 95

The PUAD/Au monolayer.................................... 100

Coverage dependance of $\mathrm{C}=\mathrm{O}$ groups............................. 103

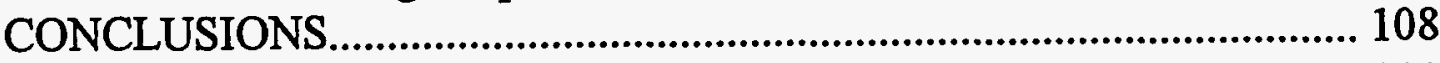

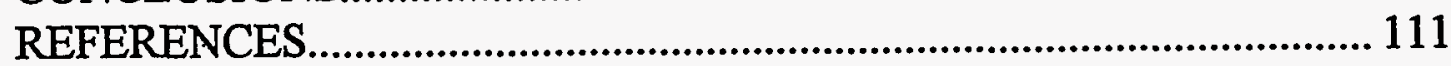

PAPER IV. INFRARED REFLECTION ABSORPTION SPECTROSCOPIC STUDIES OF THE

ACID-BASE REACTIVITY OF ORGANIC MONOLAYER FILMS: EMERSED

CARBOXYLIC ACID-TERMINATED

MONOLAYERS.

INTRODUCTION 
Monolayer preparation.................................................. 117

Emersion measurements.................................................. 118

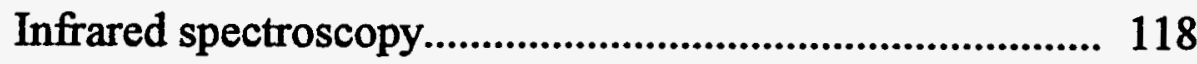

Chemicals and reagents................................................... 119

RESULTS AND DISCUSSION....................................................... 120

Methyl-terminated monolayers....................................... 124

MHA monolayers............................................................ 124

MHA monolayers with a fractional coverage of

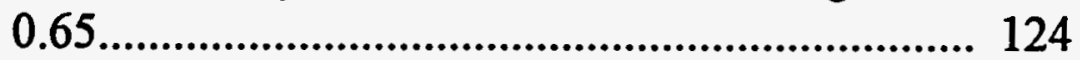

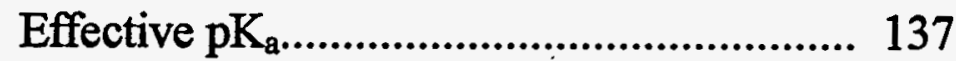

MHA monolayers with a full coverage................. 141

MUA monolayers....................................................... 148

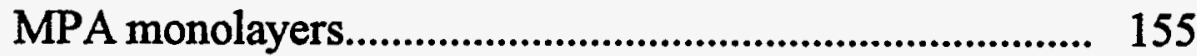

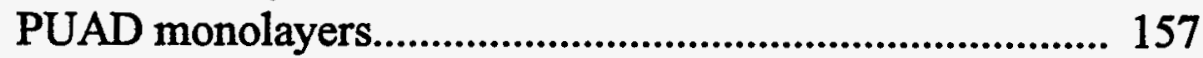

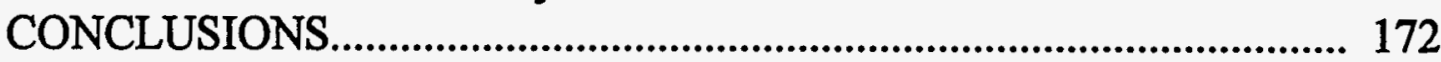

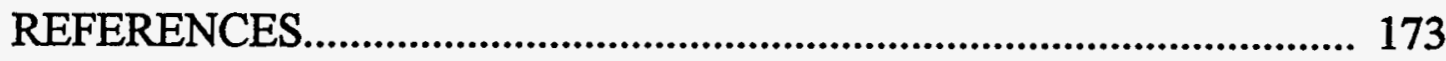

PAPER V. STUDY OF FORMATION AND

ESTERFICATION OF 11-

MERCAPTOUNDECANOIC

ACID MONOLAYERS AT GOLD USING

IRRAS AND CONTACT ANGLE

MEASUREMENT.................................... 177

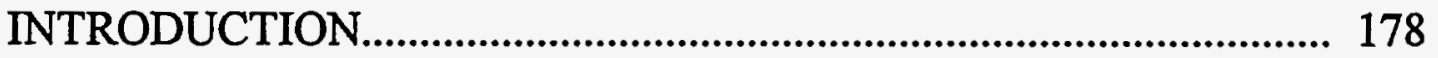

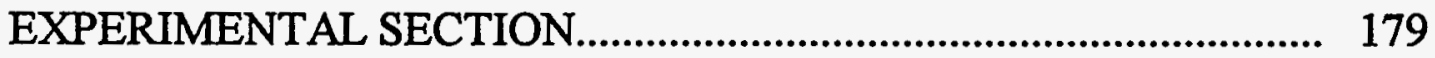

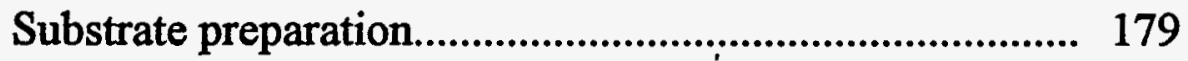

Monolayer preparation................................................. 179

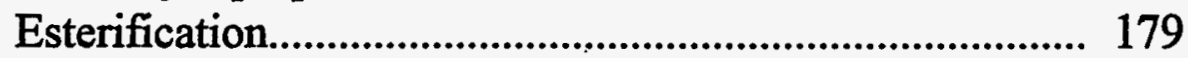

Ellipsometric measurements of films thickness................ 180

Contact angle measurements........................................... 180

Infrared spectroscopy..................................................... 180

Chemicals and reagents................................................... 181

RESULTS AND DISCUSSION.................................................. 182

Monolayer formation......................................................... 182 
Ellipsometric thickness as a function of immersion time ...................................................... 182

Wetting as a function of immersion time................ 182

IRRAS spectra as a function of immersion time.... 185

IRRAS characterization.................................................... 185

The average tilt angle of the polymethylene chain.. 185

The average tilt angle of carbonyl dipole................ 188

Three carbonyl modes............................................ 195

Esterfication of MUDA/Au monolayers.......................... 196

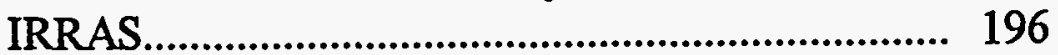

Contact angle measurements................................ 201

Kinetics analysis................................................ 201

IRRAS........................................................ 201

Contact angle measurements....................... 204

CONCLUSIONS 208

REFERENCES

209

GENERAL SUMMARY AND PROSPECTS.................................. 211

ACKNOWLEDGMENTS............................................................ 213 


\section{GENERAL INTRODUCTION}

\section{Dissertation Organization}

This dissertation consists of five research papers followed by a general summary and prospective.

\section{Overview}

The attractive properties of self-assembled monolayers including simple fabrication, stability, well-defined structures, and flexible synthetic modification have attracted enormous worldwide attention for the last decade. This interest is due largely from their potential and sometimes demonstrated use as models of organic interfaces.

The use of self-assembled monolayers is primarily controlled by their surface properties and reactivities which are dictated by the coverage, structure, dielectirc, spatial orientation, and composition of these films. Therefore, it is essential to fully understand how these factors affect the surface properties and reactivities of these films. However, there is little work has been done in this regard. The main goal of this study is to probe these relationships between these factors and the surface properties of these films to construct desired materials more effectively.

Although it has been pointed out that the adsorption of thiolate monolayers on $\mathrm{Au}$ is a two-step process using the electrochemical quartz micro balance experiment and contact angle measurement, little work has been done concerning the details of this fundamental formation process.

In Paper I, the $\mathrm{pH}$ dependence of the electron transfer of ferricyanide at phenylcarboxylic acids and pyridylcarboxylic acids adsorbed at Pt electrodes is used to determine the surface $\mathrm{pK}_{\mathrm{a}}$ of the coatings. The results of this measurement, which relies on the change in the potential at the electrode reaction plane via adsorbate deprotonation, are compared to those from contact angle titrations. Unexpected 
differences in the reactivity of the adsorbate (as opposed to their solution analogs) are discussed.

In Paper II, the formation of alkane and acid-terminated thiolate monolayers on gold is examined using infrared reflection absorption spectroscopy (IRRAS). The discovery of agostic bonds at $2903 \mathrm{~cm}^{-1}$ due to the interaction between methylene groups and a bare Au surface based on the shift in energy of the $v_{\mathrm{a}}\left(\mathrm{CH}_{2}\right)$ from $2926 \mathrm{~cm}^{-1}$ to $2903 \mathrm{~cm}^{-1}$ and the absence of the agostic bond at $2903 \mathrm{~cm}^{-1}$ on the Au surface which was pretreated with a sulfur monolayer is reported. The change in the orientation of the polymethylene chain as a function of coverage, which is based on the fact that the presence of the $v_{\mathrm{s}}\left(\mathrm{CH}_{2}\right)$ is at the expense of the amount of $v_{\mathrm{a}}\left(\mathrm{CH}_{2}\right)$, is described.

In Paper III, the coverage dependence of the amount of the nonhydrogen-bonded, the hydrogen-bonded dimeric, and the hydrogenbonded polymeric $\mathrm{C}=0$ groups of a 16-mercaptohexadecanoic acid $\left(\mathrm{HO}_{2} \mathrm{C}\left(\mathrm{CH}_{2}\right)_{15} \mathrm{SH}\right)$ on Au monolayer, which is evidenced by the fact that decrease in peak area of the hydrogen-bonded dimeric $r(\mathrm{C}=0)$ is almost equal to the total increase in peak area of the other two peaks, is studied with ellipsometry, IRRAS, contact angle measurements techniques.

In Paper IV, the surface $\mathrm{pK}_{\mathrm{a}}$ values of the non-hydrogen-bonded, the hydrogen-bonded dimeric, and the hydrogen-bonded polymeric $\mathrm{C}=\mathrm{O}$ groups of several carboxylic acid-terminated thiolate monolayers on $\mathrm{Au}$ are examined with IRRAS as a function of solution $\mathrm{pH}$ and calculated based on the $\mathrm{pH}$ where $50 \%$ of the decreases in the peak areas of the corresponding $\downarrow(\mathrm{C}=0)$ peaks.

In Paper $V$, the extent of esterification, $\theta$, of 11mercaptoundecanoic acid on Au monolayers was examined and calculated based on the decrease in the IRRAS peak area of the $v(\mathrm{C}=0)$ mode of the carboxylic acid or the increase in that of the $\forall(\mathrm{C}=0)$ mode of the ester and on the increase in contact angles. The coverage-dependent rate constant of the surface esterification reaction is discussed. 


\section{PAPER I}

ELECTRON TRANSFER OF FERRICYANIDE AT ORGANIC CARBOXYLIC ACIDS ON PLATINUM SURFACES 


\section{INTRODUCTION}

Enormous attention has been paid to molecular films because they have many very promising properties for fundamental and technological applications ${ }^{1-4}$. The component, structure and orientation of these films can affect their properties. In the densely-packed monolayer, each molecule experiences not only a highly concentrated but also well-organized environment. It is important to understand how these factors affect the properties of these films. In many instances, the reactivities of the immobilized species are markedly different from those of their solution analogs. The main focus of this study is to see how the $\mathrm{pK}_{\mathrm{a}}$ values of carboxylic acids tethered to $\mathrm{Pt}$ electrodes via aromatic moieties are affected upon immobilization. The other focus is to understand how the potential at the electrode double layer is changed due to the deprotonation of the carboxylic acid-terminated monolayer.

The packing density, structure, and orientation of a variety of aromatic species formed as monolayers at $\mathrm{Pt}$ have been extensively characterized by Hubbard and co-workers ${ }^{5-19}$ and other groups ${ }^{20-29}$. All these properties are a function of the solution concentration and depend on the nature of molecules as well. The orientation of the aromatic ring of some aromatic molecules can be parallel or vertical to the Pt surfaces at low or high concentration, respectively ${ }^{6-9}$. Some aromatic molecules can only form flat orientation ${ }^{18}$. In this study, there are two different kinds of carboxylic acids studied: phenylcarboxylic acids and pyridylcarboxylic acids. The aromatic ring of phenylcarboxylic acids are oriented parallel to the Pt surface.

On the other hand, pyridylcarboxylic acids form stable monolayers on Pt surface and orient differently from phenylcarboxylic acids ${ }^{30}$. Based on Auger electron spectroscopy (AES) data, the tilt angles of the aromatic rings of the nicotinic acid and isonicotinic acid on Pt were calculated to be $19^{\circ}$ and $15^{\circ}$ off the surface normal, respectively ${ }^{30}$. The observed packing for the $\mathrm{NA} / \mathrm{Pt}$ and INA/Pt were calculated to be $3.8 \times 10^{-10} \mathrm{~mol} / \mathrm{cm}^{2}$ and $4.2 \times 10^{-10}$ $\mathrm{mol} / \mathrm{cm}^{2}$, respectively ${ }^{30}$. This provides a very good opportunity to see how the orientation and component affect the surface properties of monolayers. 
In this paper, we report the results of a characterization of carboxylic acid functionalized monolayers (i.e. phenylcarboxylic acids:

$\mathrm{C}_{6} \mathrm{H}_{5}\left(\mathrm{CH}_{2}\right)_{\mathrm{n}} \mathrm{COOH}, \mathrm{n}=0$ to 3; pyridylcarboxylic acids:

$\left.\mathrm{C}_{5} \mathrm{NH}_{4}\left(\mathrm{CH}_{2}\right)_{n} \mathrm{COOH}, \mathrm{n}=0,1\right)$ at Pt using tow techniques: electrochemistry, and contact angle measurements (CA). The electrochemical measurements relies on the influence of the change of the charge at the electrode reaction plane as a function of the $\mathrm{pH}$-dependent ionization of the surface bond carboxylic acid groups. These changes are followed through measurements of the apparent heterogeneous electron-transfer rate constant of the solutionbases redox couple $\mathrm{Fe}(\mathrm{CN})_{6}^{-4 / 3}$. For comparison purposes, the ionization of these layers were monitored using $\mathrm{CA}$. The effects of immobilization on reactivity are discussed. 


\section{EXPERIMENTAL SECTION}

\section{Electrode preparation}

The 0.15-cm-diameter platinum electrodes (Bioanalytical System, West Lafayette, IN) and a 2" x 1" Pt foil (Engelhard Minerals \& Chem. Corp., Carteret, NJ) were smoothed to a mirror-like finish using successively fine grades of wet emery paper, followed by slurries of 1.0,0.3 and 0.05-mm diamond polishing compound (Buehler, Lake Forest, $\Pi$ ). Between each step, the electrodes were rinsed thoroughly with deionized water. The finial pretreatment step consisted of scanning the applied voltage at $100 \mathrm{mV} / \mathrm{s}$ repetitively between +1.2 and $-0.2 \mathrm{~V}$ until a steadystate voltammetric response that exhibited the characteristic hydrogen adsorption/desorption and oxide formation/desorption fine structure was obtained (see Figure 1). Electrodes so prepared will be referred to simply as an uncoated platinum electrodes. $\mathrm{An} \mathrm{Ag} / \mathrm{AgCl}$ (sat'd) reference electrode and a Pt auxiliary electrode were used. All the potentials are reported with respect to this silver reference electrode.

The active surface of the platinum electrode was determined by the charge consumed in the hydrogen atom underpotential deposition region ${ }^{11}$. The voltammetric response of an electrode coated with a layer of adsorbed iodine provided the base line for charge integration ${ }^{11}$. A value of $210 \mathrm{mC} / \mathrm{cm}^{2}$ was used as representative for the monolayer coverage of hydrogen at polycrystalline platinum ${ }^{11}$. This analysis yielded a roughness factor, which is given as the active surface area divided by the geometric surface area, of $1.3 \pm$ 0.1 . The uncertainty of the roughness factor is based on repetitive testing of five separate electrodes using the described polishing and electrochemical pretreatment methods.

\section{Monolayer preparation}

The electrodes were coated with each of the aromatic moieties by terminating a voltammetric scan in $1.0 \mathrm{M} \mathrm{H}_{2} \mathrm{SO}_{4}$ aqueous solution in the region of oxide formation. The electrode was disconnected from the potentiostat, and the electrolytic solution changed to a known volume of $1.0 \mathrm{M} \mathrm{NaF}$ solution (pH 3.0). After reconnection to the potentiostat, the voltage was stepped to 
$0.0 \mathrm{~V}$. A small volume of concentrated adsorbate was then added to the solution with a micropipette to yield the targeted concentration. Film formation was essentially complete within $5 \mathrm{~min}$ after injection, as indicated by loss of the hydrogen underpotential deposition waves, longer adsorption times were used for convenience. Over a ten minute time period, and uncoated platinum platinum electrode exhibited only a marginal loss $(<5 \%)$ of in the area of the electrode active toward hydrogen adsorption.

\section{Instrumentation and measurements}

Electrochemistry

A conventional three electrode electrochemical cell with a LugginHarber capillary and a Cypress system CY-1 computer controlled potentiostat (Cypress Systems Inc., Lawrence, KS) were used in all electrochemical experiments.

The heterogeneous electron-transfer rate constant, $k_{\text {app }}$, of ferriferrocyanide at the monolayer-coated electrodes was determined by cyclic voltammetry according to the method of Nicholson ${ }^{33}$. The working solution were $1.0 \mathrm{mM}$ ferrocyanide with $0.50 \mathrm{M} \mathrm{K}_{2} \mathrm{SO}_{4}$ as the supporting electrolyte.

For the determinations, the following constants were used for the redox couple: $D_{\text {ox }}=7.63 \times 10^{-6} \mathrm{~cm}^{2} / \mathrm{s}^{34}, D_{\text {red }}=6.32 \times 10^{-6} \mathrm{~cm}^{2} / \mathrm{s}^{34}, \alpha=0.5$, and $\mathrm{n}=$ 1.0. With these constants and the separation of the cathodic and anodic peak current potentials, $k_{\text {app }}$ was calculated as $k_{\text {app }}=2.78 \times 10^{-2} v \psi^{1 / 2}(\mathrm{~cm} / \mathrm{s})$, where $\nu$ is the scan rate and $\psi$ was taken from a working curve $e^{35}$ or from a numerical data $^{36}$.

\section{Contact angles}

Advancing contact angles $\left(\theta_{\mathrm{a}}\right)$ were measured in air with $0.100 \mathrm{~N}$ of $\mathrm{HF} / \mathrm{NaF}$ aqueous buffer solutions as probe liquids by using a Rame-Hart Model 100-00 115 goniometer. For these measurements, a $2.00 \mathrm{~mL}$ droplet was formed on the substrate with the needle of the syringe in the droplet. The value of $\theta_{\mathrm{a}}$ was determined as the volume of the droplet was slowly increased. The standard deviation of these data is $\sim 10 \%$, a reflection of the relatively small values of the contact angle. 


\section{Chemicals and reagents}

All the organic compounds including benzoic acid (BA), 2-

phenylacetic acid (PAA), 3-phenylpropanoic acid (PPA), 4-phenylbutric acid (PBA), isonicotinic acid (INA), nicotinic acid (NA), pyridylacetic acid (PYAA) (Aldrich, Milwaukee, WI) and inorganic chemicals (Fisher Scientific, Pittsburgh, PA) were reagent grades and were used without further purification. All solutions were prepared daily with purified water from a Mili-Q system (Millipore, Bedford, $\mathrm{MA}$ ). The $\mathrm{pH}$ was adjusted by adding appropriate amount of $\mathrm{H}_{2} \mathrm{SO}_{4}$ or $\mathrm{KOH}$. Solution were deoxygenated by purging with high purity argon gas for $20 \mathrm{~min}$ prior to each electrochemistry experiment and were kept under an argon atmosphere during the experiments. 


\section{RESULTS AND DISCUSSION}

\section{Characterization of monolayers}

Figure 1 shows cyclic voltammograms for an electrochemically pretreated platinum electrode (solid curve) and for platinum electrodes coated by immersion of a platinum electrode in solution of different concentration of $\mathrm{BA}$ (dashed curves). The voltammograms were obtained by sweeping the voltage at $100 \mathrm{mV} / \mathrm{s}$ from $+0.2 \mathrm{~V}$ to a lower limit of $-0.2 \mathrm{~V}$, to an upper limit of $+1.5 \mathrm{~V}$, and then returning to the initial voltage. The curve for the uncoated electrode exhibits both the hydrogen adsorption and desorption waves and the oxide formation and reduction waves characteristic of a platinum surface free of strongly bond organic adsorbates ${ }^{11}$. The presence of adsorbed BA is evident by the loss of the hydrogen adsorption/desorption waves as well as by the anodic current that flows at large positive applied voltages $(\sim+1.3 \mathrm{~V})$. The adsorbed layer blocks access to sites for hydrogen atom adsorption, and hinders the oxidation of the platinum surface. The broad anodic wave corresponds to oxidation of the surface-bonded layer. For all the acids, the charge under this anodic wave increases with increases in the concentration of the aromatic acids as shown in Figure 1.

The values for the oxidation charge were determined by integrating the charge passed at the coated electrode, from which the value over the same potential region of an uncoated electrode was subtracted. The oxidation charge density $\mathrm{Q}_{\mathrm{ox}}$ is calculated by dividing the value of the oxidation charge by the real electrode surface area which is equal to the geometric area of the electrode multiplied by the roughness factor (1.3). The dependencies of $\mathrm{Q}_{\mathrm{ox}}$ on the concentration of each of the aromatic acids are shown in Figure 2. In each instance, the value of $Q_{o x}$ increases as the adsorbate precursor concentration increases, reaching limiting values between 0.05 and $1.0 \mathrm{mM}$ depending on the acids as shown in Table 1.

The apparent surface coverage for BA and the other adsorbed layers were estimated for the limiting values based the charge density, $Q_{o x}$, consumed during the anodic sweep in the voltammotry experiments. The values of $Q_{0 x}$ for $B A, P A A, P P A$, and PBA at saturation coverages on platinum are $8.2 \times 10^{-4}, 9.5 \times 10^{-4}, 9.7 \times 10^{-4}$, and $7.9 \times 10^{-4} \mathrm{C}^{-\mathrm{cm}^{2}}$, 


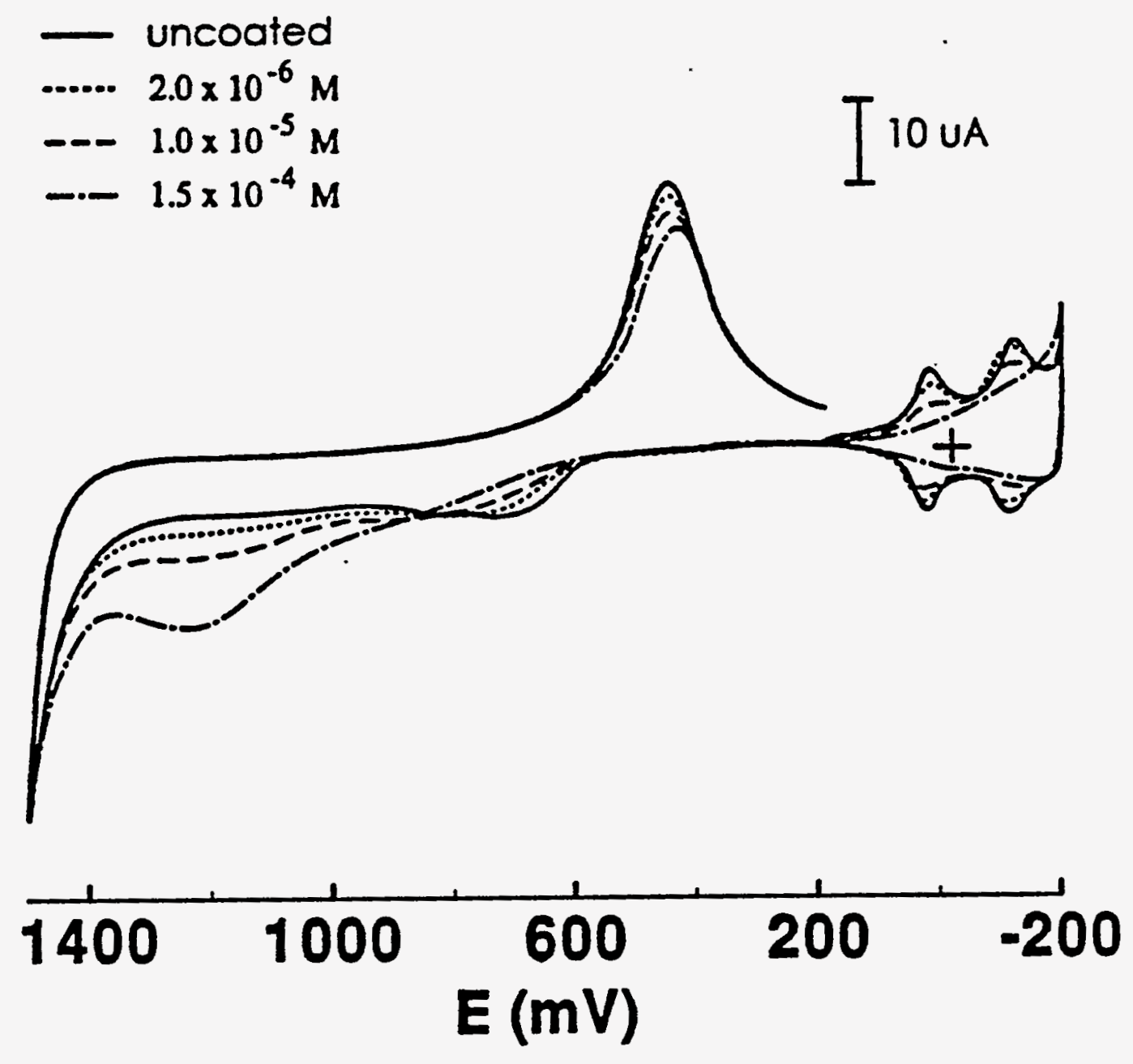

Figure 1. Cyclic voltammograms of a clean Pt electrode (solid curve) and the BA/Pt electrodes adsorbed in $2.0 \times 10^{-6} \mathrm{M}, 1.00 \times 10^{-5} \mathrm{M}$, and $1.50 \times 10^{-4} \mathrm{MBA}$ solution for $5 \mathrm{~min}$ (dashed curves) in 1.0 $\mathrm{N} \mathrm{H}_{2} \mathrm{SO}_{4}$ aqueous solution at $100 \mathrm{mV} / \mathrm{s}$ scan rate. 


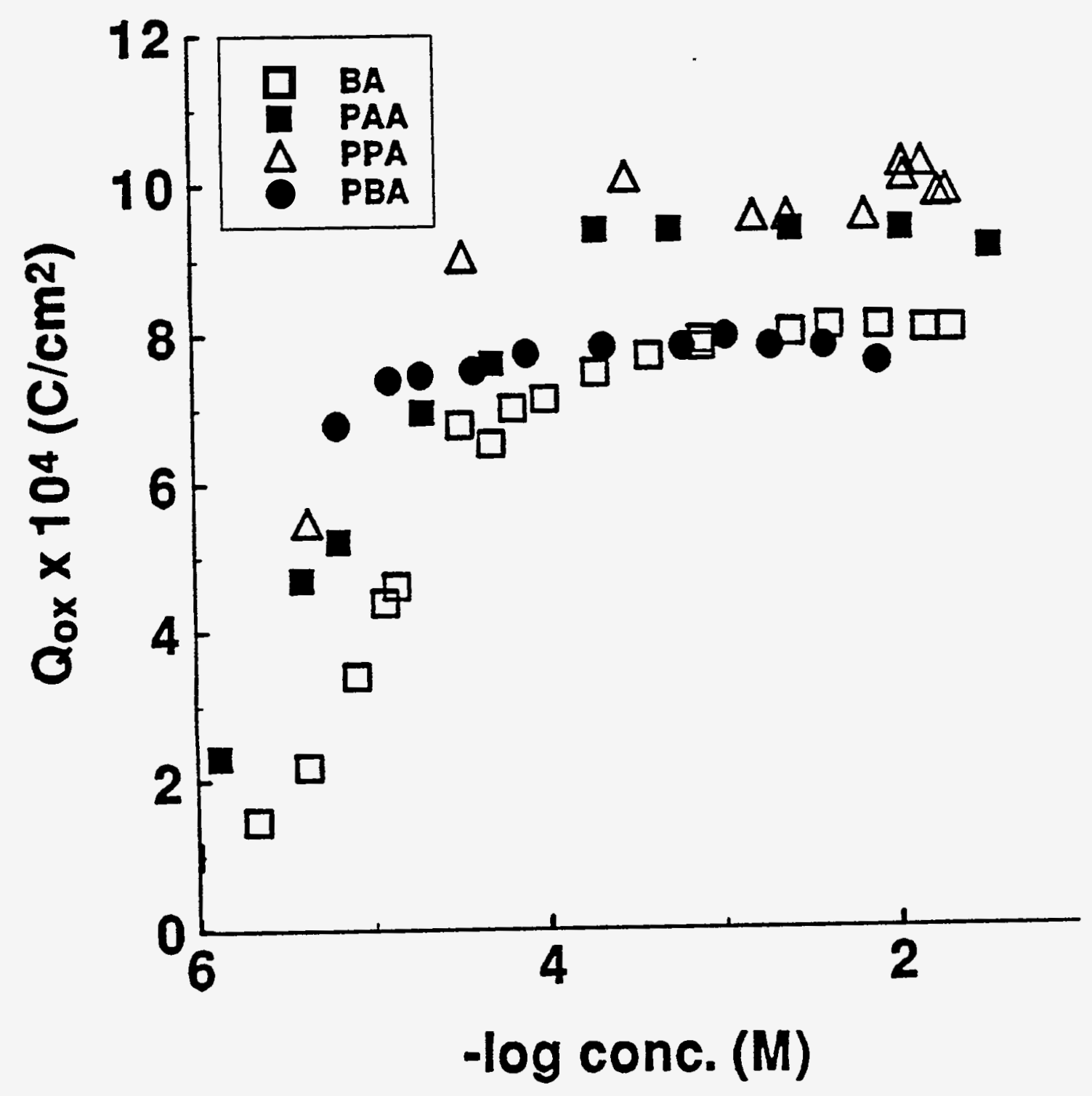

Figure 2. The $\mathrm{Q}_{\mathrm{ox}}$ of $\mathrm{BA}, \mathrm{PAA}, \mathrm{PPA}$, and $\mathrm{PBA}$ on platinum electrode as a function of - $\log$ concentration. 
Table 1. Oxidative stripping charge density $\left(\mathrm{Q}_{\mathrm{ox}}\right)$, and saturation surface coverage estimates experimental $\left(\Gamma_{\mathrm{ox}}\right)$ and theoretical $\left(\Gamma_{\text {calc }}\right)$ at limiting concentrations of aromatic acid layers at platinum electrodes.

\begin{tabular}{lcccc}
$\begin{array}{l}\text { adsorbate } \\
\text { precursor }\end{array}$ & $\begin{array}{c}\mathrm{Q}_{\mathrm{ox}} \times 10^{4} \\
\left(\mathrm{C} / \mathrm{cm}^{2}\right)\end{array}$ & $\mathrm{n}_{\mathrm{ox}}$ & $\begin{array}{c}\Gamma_{\text {ox }} \times 10^{10} \\
\left(\mathrm{~mol} / \mathrm{cm}^{2}\right)\end{array}$ & $\begin{array}{c}\Gamma_{\text {calc }} \times 10^{10} \\
\left(\mathrm{~mol} / \mathrm{cm}^{2}\right)\end{array}$ \\
\hline BA & $8.2 \pm 10$ & 30 & 2.8 & 2.9 \\
PAA & $9.5 \pm 10$ & 36 & 2.8 & 2.8 \\
PPA & $9.7 \pm 10$ & 42 & 2.4 & 2.5 \\
PBA & $7.9 \pm 10$ & 48 & 1.7 & 2.3 \\
\hline
\end{tabular}


respectively with an error bar of $0.1 \times 10^{-4} \mathrm{C}_{\mathrm{cm}} \mathrm{cm}^{2}$. Saturation coverages, $\Gamma_{\mathrm{ox}}$, from these measurements are calculated as

$$
\Gamma_{o x}=Q_{o x} / n_{o x} F
$$

where $\mathrm{n}_{\mathrm{ox}}$ is the number of electrons required to oxidize the adsorbed layer exhaustively to $\mathrm{CO}_{2}$ (e.g., $\mathrm{C}_{6} \mathrm{H}_{5} \mathrm{CO}_{2} \mathrm{H}+12 \mathrm{H}_{2} \mathrm{O}>7 \mathrm{CO}_{2}+30 \mathrm{H}^{+}+30$ e), $\mathrm{F}$ is the Faraday ${ }^{17}$. In addition to the exhaustive electrolysis to $\mathrm{CO}_{2}$, there are two further assumptions: 1) the adsorbate orientation and/or mode of attachment is constant over the concentration range studied; and 2) the composition of the adsorbate is the same. The limitation of this approach have been examined in depth by Hubbard and co-workers ${ }^{19}$; our intent, however, is to use this as a qualitative indicator of coverage and orientation. The errors in measurement arise from the assumption that oxidation of adsorbed species always proceeds completely to $\mathrm{CO}_{2}$. Complete conversion of adsorbed aromatics occurs only for flat-adsorbed molecules. In other words, this method cannot be applied to the edge-adsorbed molecules because these adsorbed molecules cannot be oxidized exhaustively to $\mathrm{CO}_{2}$. However, this method can be used to differentiate a flat-adsorbed molecule from an edge-adsorbed molecule.

The values of $\Gamma_{0 x}$ for BA, PAA, PPA, and PBA are $2.8 \times 10^{-10}, 2.8 \times 10$ ${ }^{-10}, 2.4 \times 10^{-10}$, and $1.7 \times 10^{-10} \mathrm{~mol} / \mathrm{cm}^{2}$, respectively. The results of the coverage analyses are listed in Table 1 along with coverage calculated for the closest-packed model structures for each adsorbate, $\Gamma_{\text {calc. }}$. The values for $\Gamma_{\text {calc }}$ were determined based on a fully extended alkyl chain with the plane of the aromatic ring oriented parallel to the Pt surface, i.e. in the $\eta 6$ orientation ${ }^{6,37,38}$. The $\Gamma$ for the closest-packed model structures with the plane of the aromatic ring oriented vertical to the $\mathrm{Pt}$ surface is not used because its value $(\sim 7.0 \times 10$ ${ }^{-10} \mathrm{~mol} / \mathrm{cm}^{2}$ ) is $\sim 3$ times larger than the experimental values. From Table 1 , the values of $\Gamma_{\mathrm{ox}}$ for BA, PAA, and PPA are in good agreement with the corresponding $\Gamma_{\text {calc }}$ values. This suggests that the BA, PAA, and PPA form closest-packed monolayers with the aromatic rings oriented parallel to the $\mathrm{Pt}$ surface. The $\Gamma_{0 x}$ value for PBA is, however, much less than the corresponding $\Gamma_{\text {calc }}$ value, precluding an orientation analysis. 
The difference in the $\Gamma_{\mathrm{ox}}$ and $\Gamma_{\text {calc }}$ values for PBA is possibly due to the incomplete oxidation of long alkyl chain ${ }^{19}$. For the purposes of our discussion, we will nevertheless assume that the aromatic ring for PBA is oriented parallel to the Pt surface.

On the other hand, pyridylcarboxylic acid monolayers on platinum electrodes are oriented with the aromatic ring almost vertical to the Pt surface. As shown by Hubbard and co-workers, rings with a vertical orientation cannot be fully oxidized electrochemically before the occurrence of the strong oxygen evolution wave. This prohibits the same coverage analysis for all pyridylcarboxylic acids on platinum electrodes. Therefore, the packing densities of the INA of $\left(4.2 \times 10^{-10} \mathrm{~mol} / \mathrm{cm}^{2}\right)$ and NA $\left(3.8 \times 10^{-10} \mathrm{~mol} / \mathrm{cm}^{2}\right)$ monolayers on platinum electrodes calculated by Hubbard and co-workers ${ }^{30}$ were used in this study.

\section{Electrochemical characterization of acid-base reactivity}

To probe the acid-base chemistry of these monolayers, the $\mathrm{pH}$ dependence of the apparent heterogeneous rate constant $\left(k_{\text {app }}\right)$ for ferricyanide redox couple $\left(\mathrm{Fe}(\mathrm{CN})_{6}^{-4 /-3}\right)$ in solution $\mathrm{pH}$ was monitored. The underlying basis of this approach relies on the effect of the build up of a negative charge at the electrode surface as the carboxylic acid ionized as the solution $\mathrm{pH}$ increases. Values of $k_{\text {app }}$ was calculated based on the difference in potential of the anodic and cathodic peak currents from cyclic voltammograms ${ }^{33,35,36}$.(see experimental section) Figure 3 presents a portion of such data at an INA/Pt, which is summarized in Table 2. As expected, the peak separation increases as the solution $\mathrm{pH}$ increases, indicative of a decrease in $\mathrm{k}_{\mathrm{app}}$.

Plots of $k_{\text {appa }}$ for the redox couple at BA/Pt, PAA/Pt, PPA/Pt and $\mathrm{PAA} / \mathrm{Pt}$ electrodes as a function of solution $\mathrm{pH}$ are shown in Figure 4. At low $\mathrm{pH}$, the values of $\mathrm{k}_{\text {app }}$ at $\mathrm{BA} / \mathrm{Pt}, \mathrm{PAA} / \mathrm{Pt}$, and PPA/Pt are almost the same but that at PBA/Pt is slightly smaller. This indicates that the thicknesses of BA/Pt, $\mathrm{PAA} / \mathrm{Pt}$, and PPA/Pt monolayers are similar. In contrast, the lower value of $k_{\text {app }}$ for the PBA/Pt monolayer is indicative of a slightly thicker film as dictated by the electron tunneling i.e. the greater the thickness of the adsorbed monolayer the larger the decrease in $k_{\text {app. }}$. In other words, the structural 


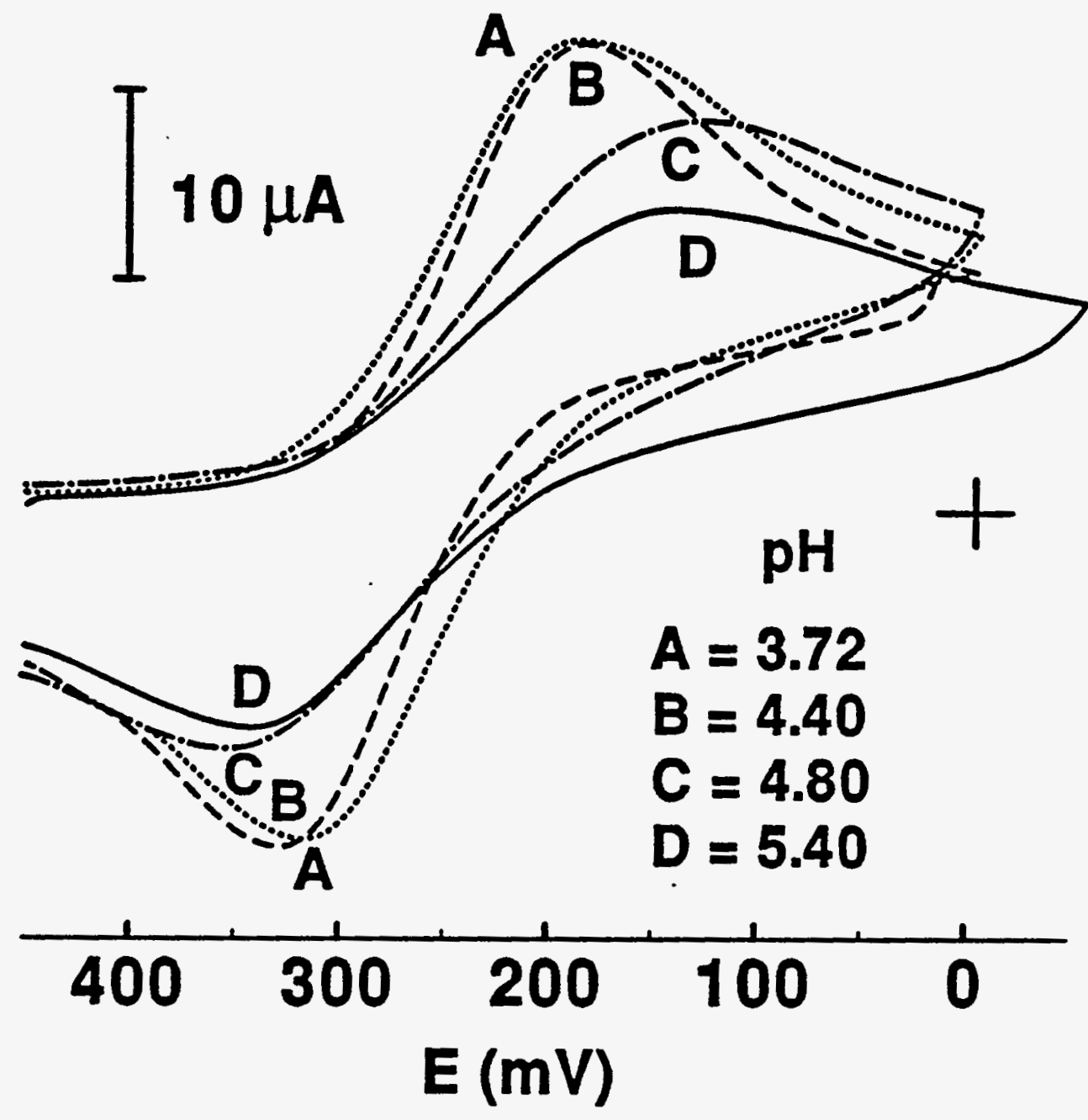

Figure 3. Cyclic voltammograms of $1.0 \mathrm{mM}$ ferricyanide at a INA/Pt electrode in $1.0 \mathrm{~N} \mathrm{~K}_{2} \mathrm{SO}_{4}$ solution at $\mathrm{pH} 3.7$ (curve $\mathrm{A}$ ), $\mathrm{pH} 4.4$ (curve B), $\mathrm{pH} 4.8$ (curve C), and pH 5.4 (curve D). 
Table 2. The peak separation $\left(\Delta E_{\text {peak }}\right)$ and $k_{\text {app }}$ for ferricyanide at INA/Pt as a function of solution $\mathrm{pH}$ and scan rate $(v)$.

\begin{tabular}{lcccc}
\hline$\Delta E_{\text {peak }}(\mathrm{mV})$ & $\mathrm{pH}=2.20$ & $\mathrm{pH}=3.72$ & $\mathrm{pH}=4.40$ & $\mathrm{pH}=5.40$ \\
\hline$v(\mathrm{~V} / \mathrm{s})=5$ & 102 & 113 & 137 & 223 \\
$v(\mathrm{~V} / \mathrm{s})=3$ & 81 & 94 & 117 & 177 \\
$v(\mathrm{~V} / \mathrm{s})=2$ & 79 & 84 & 110 & 162 \\
$\nu(\mathrm{V} / \mathrm{s})=1$ & 77 & 79 & 96 & 135 \\
\hline $\mathrm{k}_{\text {app }}(\mathrm{cm} / \mathrm{s})$ & 5.2 & 3.0 & 2.0 & 0.85 \\
\hline
\end{tabular}




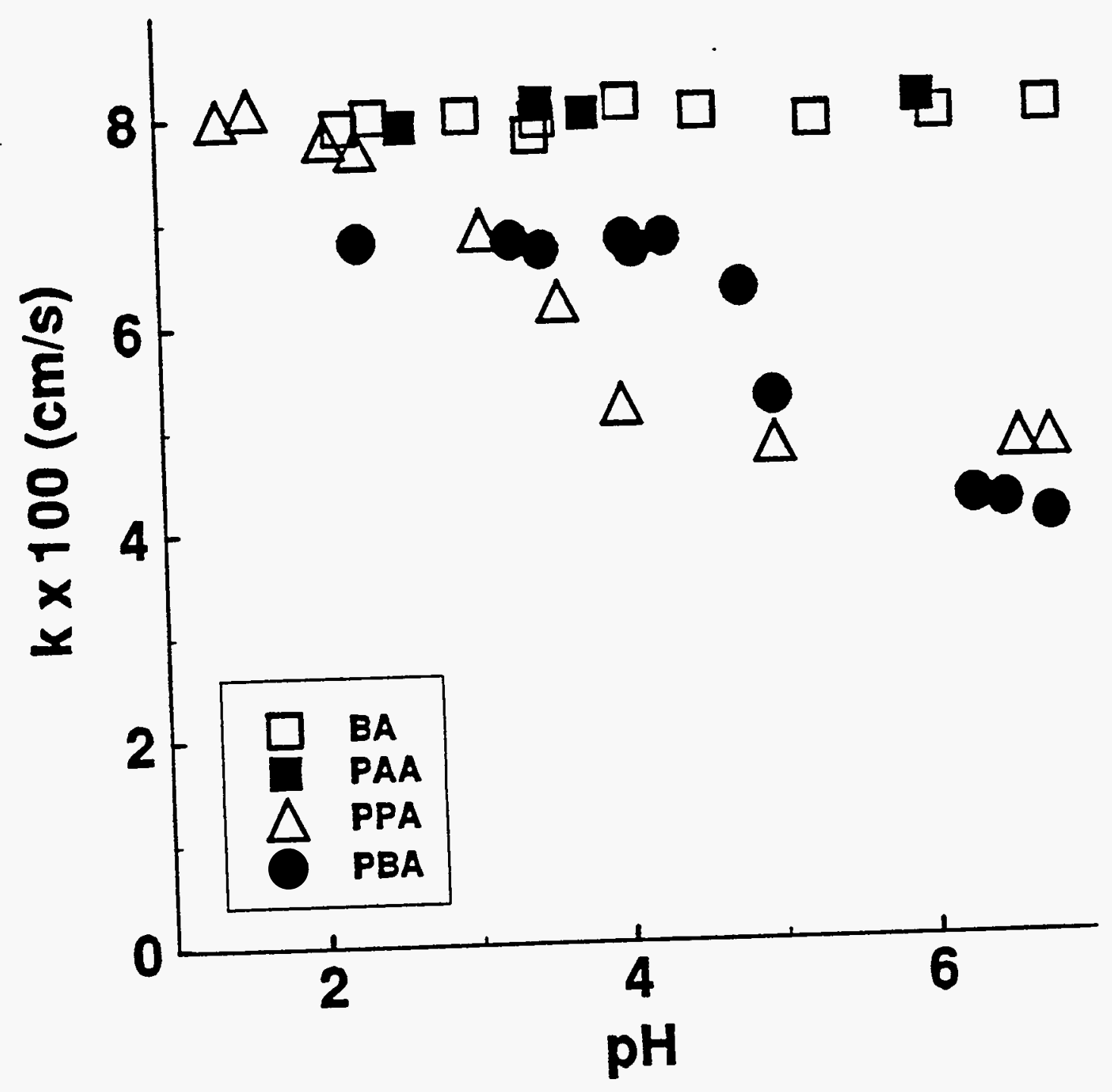

Figure 4. The $\mathrm{k}_{\mathrm{app}}$ of ferricyanide at the BA/Pt, PAA/Pt, PPA/Pt, and $\mathrm{PBA} / \mathrm{Pt}$ electrodes as a function of solution $\mathrm{pH}$. 
differences between the BA/Pt, PAA/Pt, PPA/Pt and the PBA/Pt monolayers led to differences in the plane of closest approach for ferricyanide. This is consistent with our interpretation of coverage analysis data as discussed early. The values of $k_{\mathrm{app}}$ of ferricyanide at both BA/Pt and PAA/Pt are constant within the $\mathrm{pH}$ window studied while those at PAA/Pt and PBA/Pt decrease with increasing solution $\mathrm{pH}$. The $\mathrm{k}_{\mathrm{app}}$ values at control monolayers (i.e. these without an acidic functional group, like pyridine and benzene) exhibit no observable dependence on $\mathrm{pH}$ as shown in Figure 5. Importantly, the transition in the $k_{\text {app }}$ values spans $\sim 3$ decade in hydrogen ion concentration, diagnostic of the deprotonation of an acidic functional group. Since it has been demonstrated that the carboxylic acid groups of benzoic acid can form a carboxylate-Pt bond at these electrode ${ }^{30}$ and effectively lose any acid-base chemistry at this site, we should lose acid-base reactivity. Since the $k_{\text {app }}$ of ferricyanide at BA/Pt and PAA/Pt are the same as that at protonated PPA/Pt, it is unlikely that both BA/Pt and PAA/Pt exist in their basic forms at low solution $\mathrm{pH}$. In other words, if both $\mathrm{BA} / \mathrm{Pt}$ and $\mathrm{PAA} / \mathrm{Pt}$ monolayers were strong acids because of immobilization, then $\mathrm{k}_{\text {app }}$ would be lower than observed at low $\mathrm{pH}$ (see below). More evidence and more discussion will be provided later in support our conclusion and make one believe that the carbonyl of both BA/Pt and PAA/Pt monolayers are inactive.

Plots of $\mathrm{k}_{\text {app }}$ of ferricyanide at INA/Pt, NA/Pt, and PYAA/Pt electrodes as a function of solution $\mathrm{pH}$ are shown in Figure 6. In each plot, there is a titration curve centered at around $\mathrm{pH} 4$. Since PYAA/Pt should have the largest film thickness, ferricyanide should as before have the smallest $k_{\text {app }}$ at PYAA/Pt. The thicknesses differences of the INA and NA monolayers are probably too small to influence these data to an observable extent ${ }^{39}$. This curve has a very similar shape to the one found by using IRRAS (see below). We therefore attribute the decreases in $\mathbf{k}_{\mathrm{app}}$ to the deprotonation process.

The $k_{\text {app }}$ values of ferricyanide are smaller at pyridylcarboxylic acids monolayers than that at PYR/Pt because the thicknesses of the foamier are thicker than that of the latter which does not have the carboxylic acid group at all as shown in Figure 4. Notice, the $k_{\text {app }}$ values of ferricyanide at PYR/Pt is 


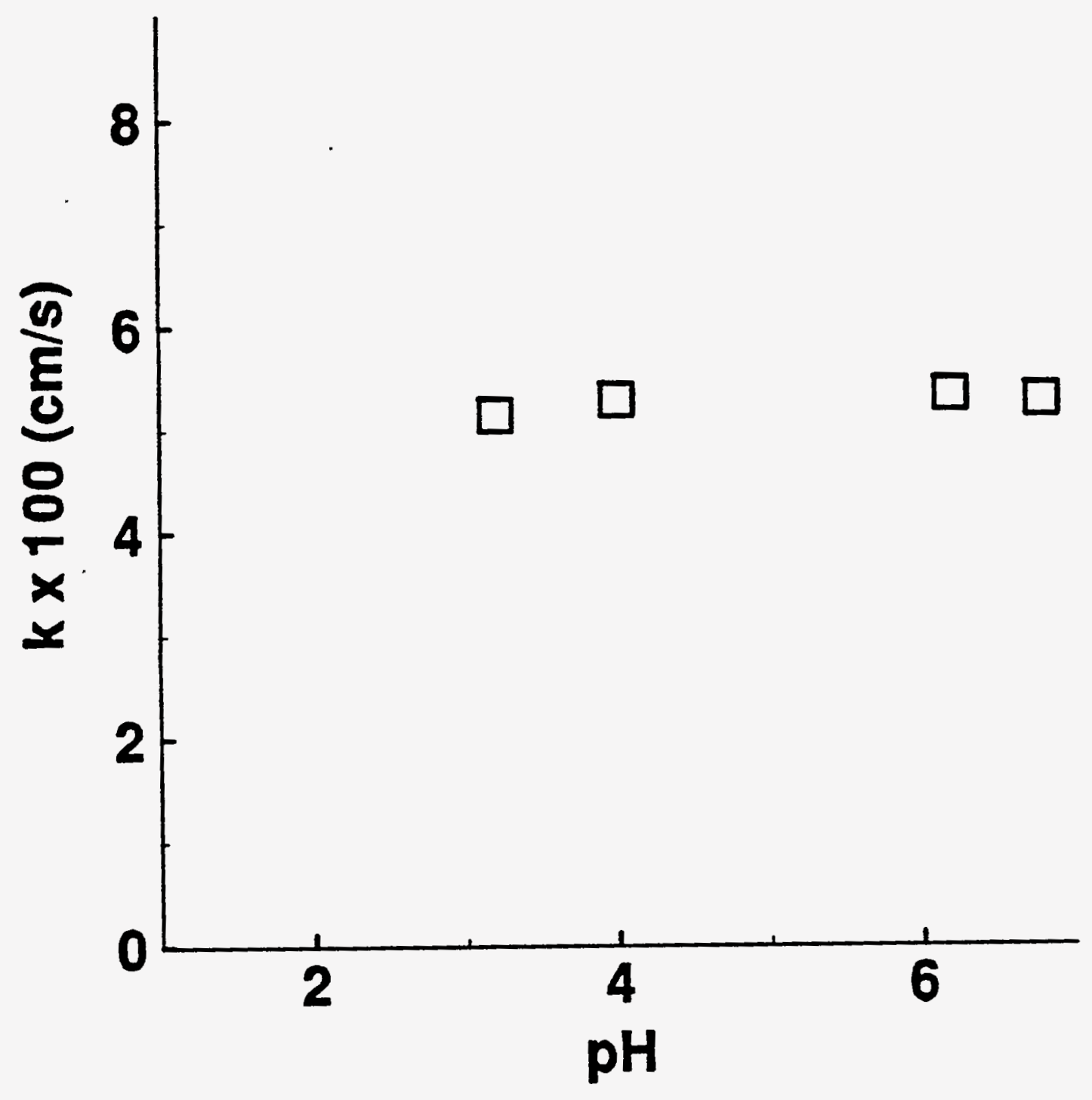

Figure 5. The $k_{\text {app }}$ of ferricyanide at the BEN/Pt electrode as a function of solution $\mathrm{pH}$. 


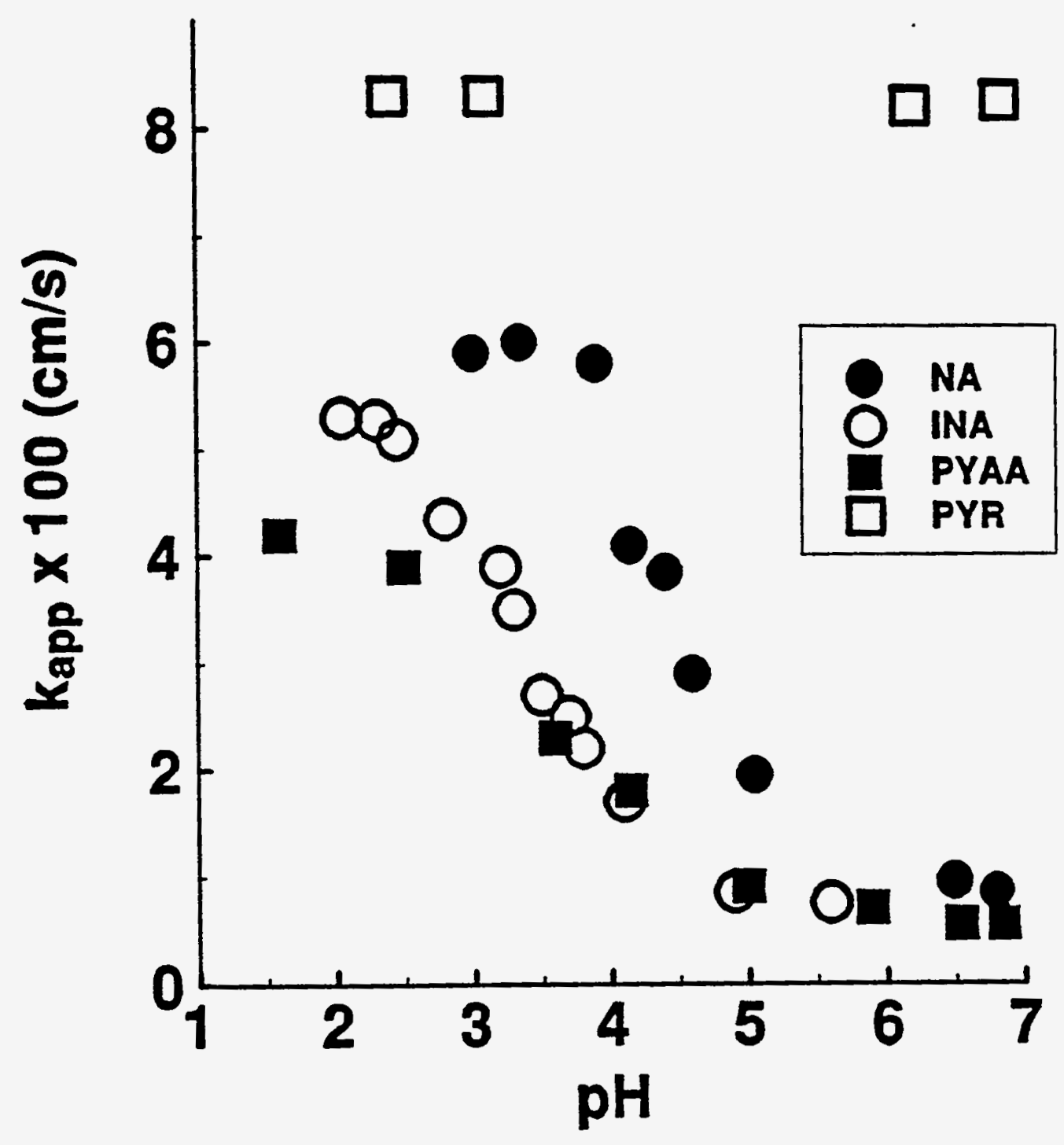

Figure 6. The $k_{\text {app }}$ of ferricyanide at the NA/Pt, INA/Pt, PYAA/Pt, and PYR/Pt electrodes as a function of solution $\mathrm{pH}$. 
independent with solution $\mathrm{pH}$.

The $k_{\text {app }}$ values of ferricyanide are smaller at pyridylcarboxylic acids monolayers than that at phenylcarboxylic acids monolayers because the thickness of the former is thicker than the that of the latter as a result of different orientation as shown in Figures 4 and 6.

The $\Delta \mathrm{k}_{\text {app }}$ is greater at phenylcarboxylic acids monolayers than that at phenylcarboxylic acids monolayers mainly because the latter has lower packing density of carboxylic acid groups and has inactive carboxylic acid groups as well.

The basis of the observed data are caused by a change in the potential at the reaction plane which can cause a change in $k_{\text {app. }}$. For acid-terminated monolayers, the potential at the reaction plane becomes negative upon deprotonation. The electrostatic repulsion between ferricyanide and the acidterminated surface upon deprotonation discourage the electron transfer rate of ferricyanide. This decrease in $k_{\text {app }}$ is a consequence of the Frumkin effect ${ }^{40-52}$ through a change in the potential in the reaction plane and is examined in detail in the next section.

\section{Potential change at the reaction plane}

The potential at the reaction plane, $\phi_{\mathrm{r}}$, in a heterogeneous electron transfer process will decrease as a result of the increase in the amount of negative charges from the carboxylate group generated by the deprotonation of the carboxylic acid group. The decrease in $\phi_{\mathrm{r}}$ causes the decrease in the rate constant of ferricyanide which has negative charge as well. The change in $\phi_{r}$ can be calculated based on the following equation from Frumkin ${ }^{31}$

$$
\Delta \ln \left(\mathrm{k}_{\mathrm{app}}\right)=(\alpha-\mathrm{z})(\mathrm{F} / \mathrm{RT})(\Delta \phi \mathrm{r})
$$

where $\alpha$ is the electron transfer coefficient, $z$ is the apparent charge on the redox species, and $F, R$, and $T$ have their conventional meanings. A value of 2 instead of -3 is used for $z$ because of the almost complete association between $\mathrm{K}^{+}$and $\mathrm{Fe}(\mathrm{CN})_{6}^{-4 / 3}$. A value of 0.5 is used for $\alpha^{53}$. From the data in Table 3, these changes in $\phi_{\mathrm{r}}$ for $\mathrm{BA} / \mathrm{Pt}, \mathrm{PAA} / \mathrm{Pt}, \mathrm{PPA} / \mathrm{Pt}, \mathrm{PBA} / \mathrm{Pt}, \mathrm{NA} / \mathrm{Pt}$, 
Table 3. The ratio of $\mathrm{k}$ of ferricyanide at $\mathrm{pH} 2\left(\mathrm{k}_{\mathrm{app} 2}\right)$ to that at $\mathrm{pH} 6$ $\left(k_{\text {appo }}\right)$, the potential change at reaction plane $\left(\Delta \phi_{\mathrm{r}}\right)$ and the theoretical potential change ( $\left.\Delta \phi_{\text {theo }}\right)$ due to deprotonation, and the ratio of these two values for the following monolayers on platinum electrodes: BA/Pt, PAA/Pt, PPA/Pt, PBA/Pt, INA/Pt, and NA/Pt.

\begin{tabular}{lcccc}
\hline monolayers & $\mathrm{k}_{\text {app2 }} / \mathrm{k}_{\text {app }}$ & $\Delta \phi_{\mathrm{r}}(\mathrm{V})$ & $\Delta \phi_{\text {theo }}(\mathrm{V})$ & $\Delta \phi_{\mathrm{r}} / \Delta \phi_{\text {theo }}$ \\
\hline BA/Pt & 1.00 & 0.00 & -0.875 & 0.0000 \\
PAA/Pt & 1.00 & 0.00 & -0.854 & 0.0000 \\
PPA/Pt & 1.67 & -0.00525 & -0.742 & 0.0071 \\
PBA/Pt & 1.61 & -0.00500 & -0.717 & 0.0070 \\
INA/Pt & 7.8 & -0.0211 & -1.76 & 0.0124 \\
NA/Pt & 6.9 & -0.0198 & -1.59 & 0.0123 \\
\hline
\end{tabular}


INA/Pt and PYAA/Pt from low to high $\mathrm{pH}$ were calculated to be $0.00 \mathrm{mV}$, $0.00 \mathrm{mV},-5.25 \mathrm{mV},-5.00 \mathrm{mV},-19.8 \mathrm{mV},-21.11 \mathrm{mV}$ and $-20.0 \mathrm{mV}$, respectively (see Table 3 ).

The theoretical $\Delta \phi_{\mathrm{r}}$ values can be calculated using the following equation

$$
\Delta \phi_{\text {theo }}=\chi \Gamma \mathrm{F} / \mathrm{c}
$$

where $\chi, \Gamma$, and $\mathrm{c}$ are the molar fraction of carboxylate, the packing density, and the capacitance (a value of $23 \mu \mathrm{F} / \mathrm{cm}^{2}$ for all except a value of 31 $\mu \mathrm{F} / \mathrm{cm}^{2}$ for $\mathrm{BA} / \mathrm{Pt}, \mathrm{PPA}$, and PPA/Pt ${ }^{31,54}$, respectively. Since there is no coverage data available for PYAA/Pt, there is no way of calculating the theoretical change in $\phi_{\mathrm{r}}$ for PYAA/Pt. The theoretical change in $\phi_{\mathrm{r}}$ for $\mathrm{BA} / \mathrm{Pt}$, PAA/Pt, PPA/Pt, PBA/Pt, INA/Pt, and NA/Pt were calculated to be $0.875 \mathrm{~V},-0.854 \mathrm{~V},-0.742 \mathrm{~V},-0.717 \mathrm{~V},-1.76 \mathrm{~V}$, and $-1.59 \mathrm{~V}$, respectively. Therefore, the observed $\Delta \phi$ values for PPA/Pt, PBA/Pt, INA/Pt and NA/Pt are significantly smaller than theoretical prediction. (see Table 3)

Other than the difference in the packing density, the difference in $\Delta \phi_{\mathrm{r}}$ mainly is a result of the difference in orientation of monolayers. The carboxylic acid groups of both BA/Pt and PAA/Pt are so close to the Pt surface that all the acid groups form carboxylate-Pt bonds which do not have any acid-base functionality at all. The carboxylic acid groups of PPA/Pt and PBA/Pt are not quite far away from the Pt surface so that part of the them are bonded to Pt surface and the rest are in acidic form. To the extreme, the carboxylic acid groups of INA/Pt, NA/Pt, and PYAA/Pt are completely away from Pt surface and therefore all of them are in acidic form. The results clearly demonstrates that the orientation of the monolayers can influence the properties of the films substantially.

The $k_{\text {app }}$ of ferricyanide as a function of solution $\mathrm{pH}$ for PPA/Pt, $\mathrm{PBA} / \mathrm{Pt}, \mathrm{INA} / \mathrm{Pt}$, and NA/Pt can be fitted with the same percentage of the theoretical $\Delta \phi_{\mathrm{r}}$ values well as shown in Figures 7-10. With only $\sim 1 \%$ of the theoretical value, the $\Delta \phi_{\mathrm{r}}$ is most likely due to the screening effect of the 


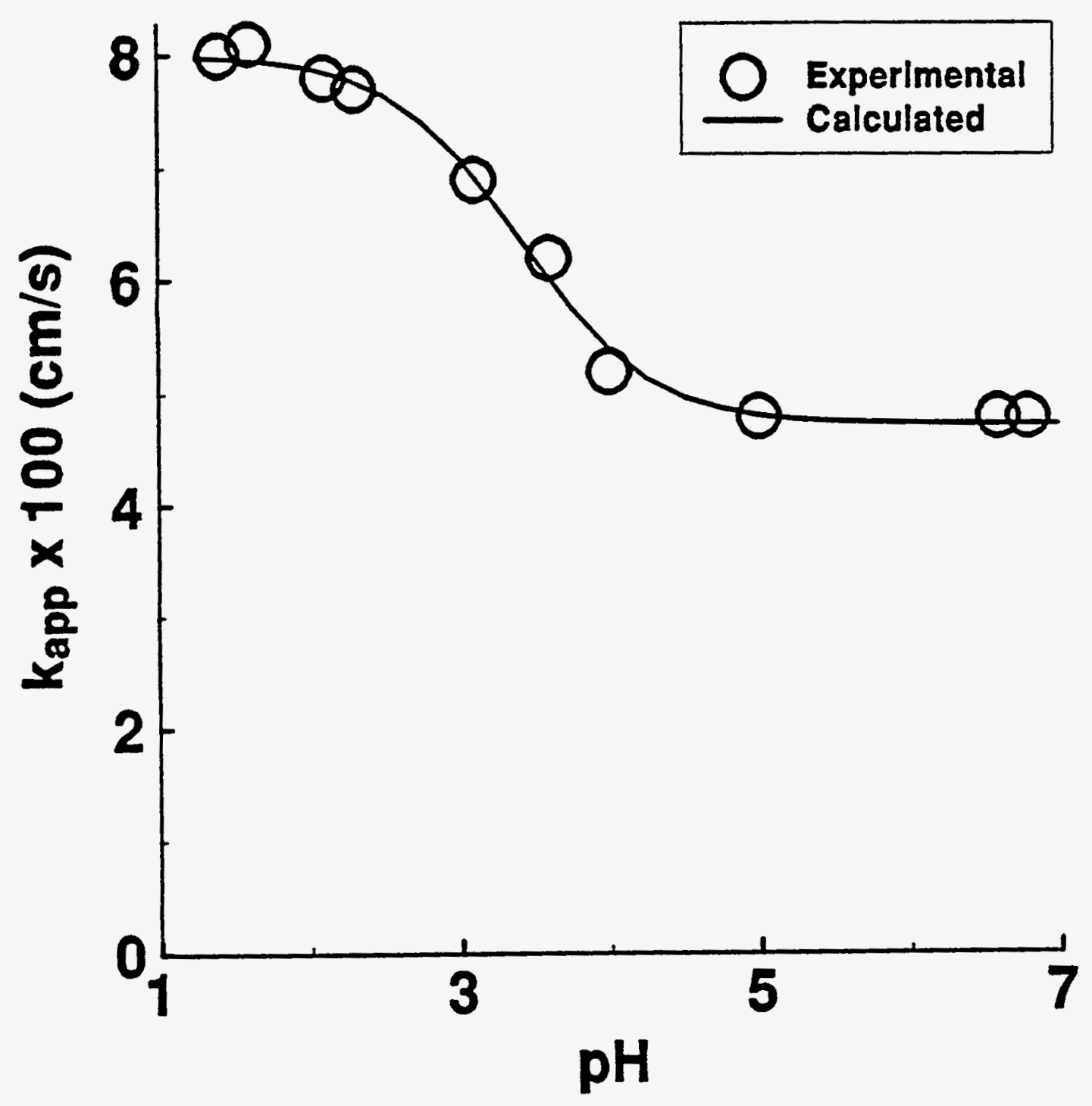

Figure 7. The $k_{\text {app }}$ of ferricyanide at the PPA/Pt electrode (open circle) and calculated $k_{\text {app }}$ using $0.71 \%$ of the theoretical $\Delta \phi$ value (solid line) as a function of solution $\mathrm{pH}$. 


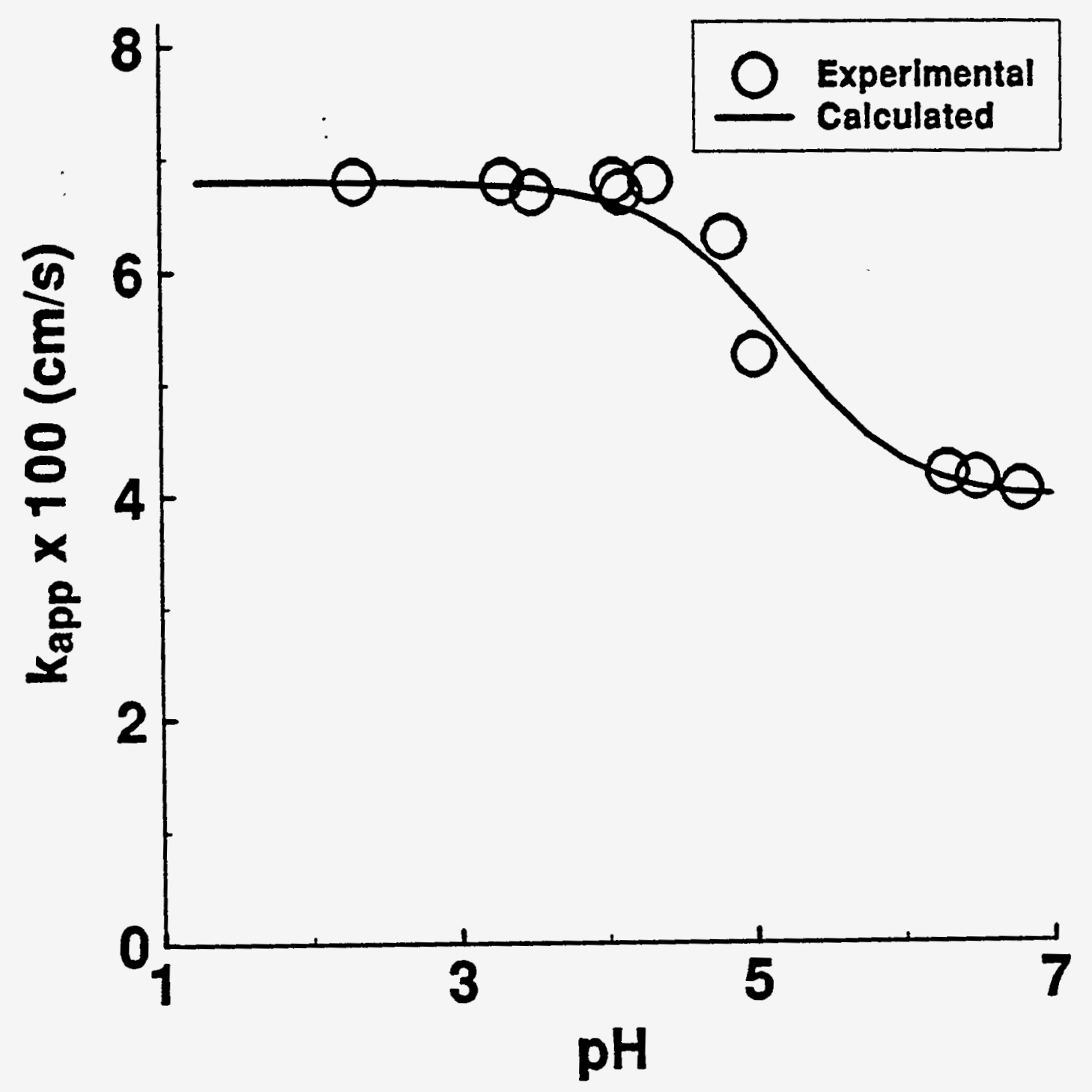

Figure 8. The $k_{\text {app }}$ of ferricyanide at the PBA/Pt electrode (open circle) and calculated $k_{\text {app }}$ using $0.70 \%$ of the theoretical $\Delta \phi$ value (solid line) as a function of solution $\mathrm{pH}$. 


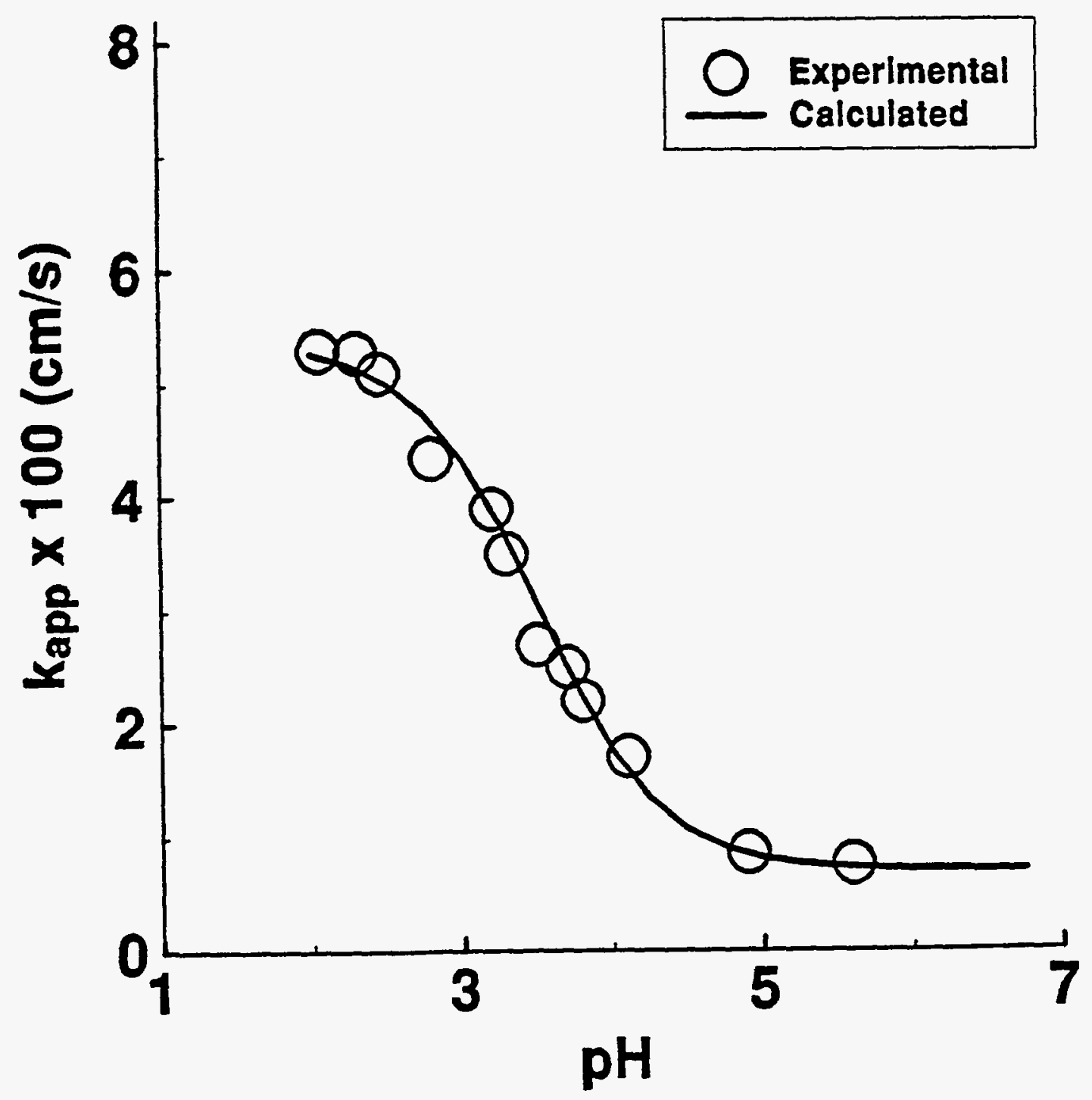

Figure 9. The $k_{\mathrm{app}}$ of ferricyanide at the $\mathrm{NNA} / \mathrm{Pt}$ electrode (open circle) and calculated $k_{\text {app }}$ using $1.2 \%$ of the theoretical $\Delta \phi$ value (solid line) as a function of solution $\mathrm{pH}$. 


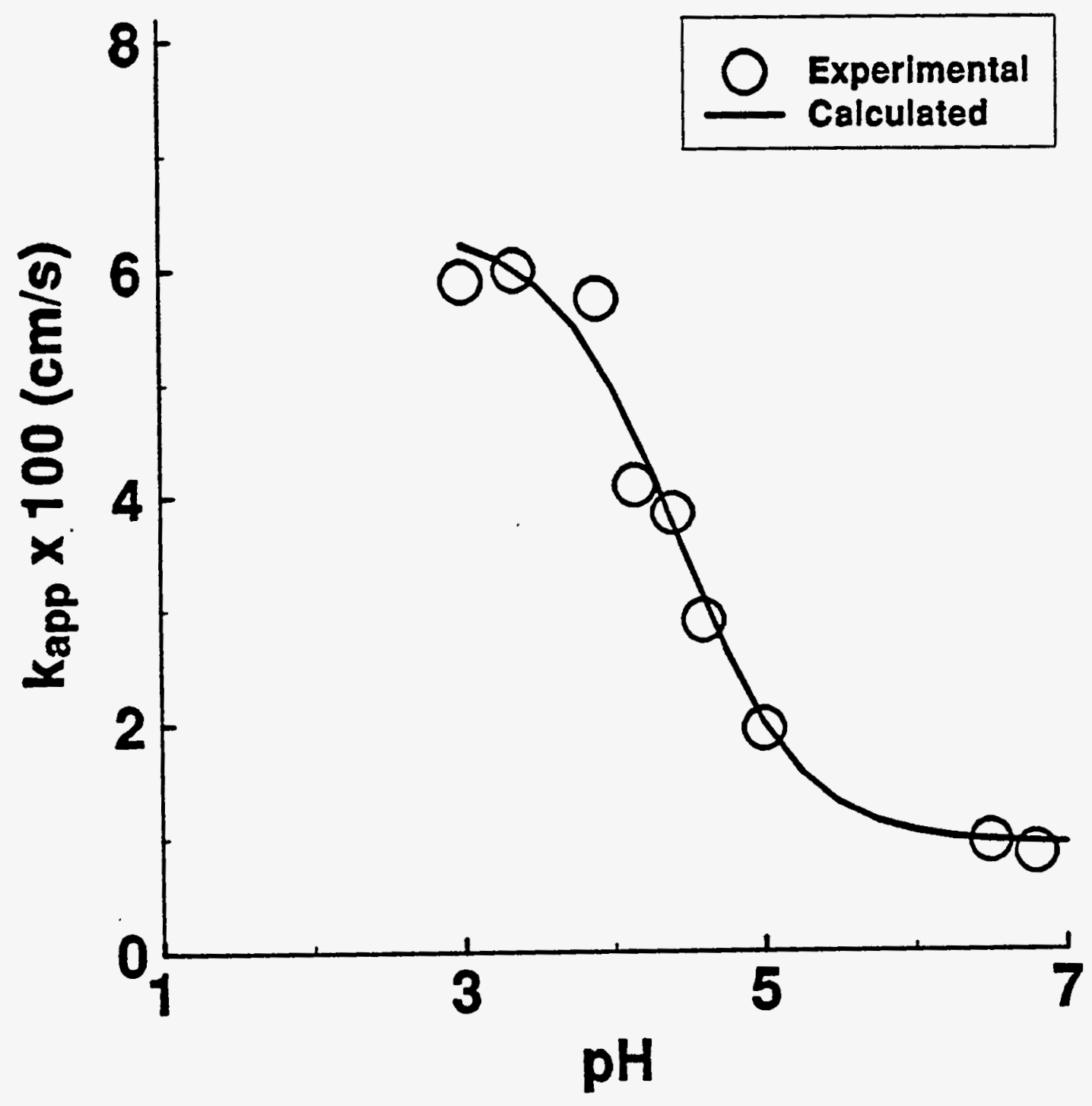

Figure 10. The $k_{\text {app }}$ of ferricyanide at the NA/Pt electrode (open circle) and calculated $k_{\text {app }}$ using $1.2 \%$ of the theoretical $\Delta \phi$ value (solid line) as a function of solution $\mathrm{pH}$. 
counter ions at the reaction plane because of the $\mathrm{COO}^{-}$group ${ }^{51,55}$ i.e. most of the negative charges at the electrode are screened by $\mathrm{K}^{+}$and $\mathrm{H}_{2} \mathrm{O}$.

\section{Determination of $\mathrm{pK}_{\mathbf{2}}$ using electrochemistry methods}

Since the decrease in $\mathrm{k}_{\mathrm{app}}$ is associated with deprotonation process, it is possible to calculate the $\mathrm{pK}_{\mathrm{a}}$ for these monolayers on Pt from the $\mathrm{k}_{\text {app }} \mathrm{vs.}$ $\mathrm{pH}$ plot. The $\mathrm{pK}_{\mathrm{a}}$ values for PPA/Pt, PBA/Pt, INA/Pt, NA/Pt and PAA/Pt were calculated based on this plot by using equation (1) at $50 \%$ of theoretical $\Delta \phi_{\mathrm{r}}$ values to be $3.5 \pm 0.3,5.3 \pm 0.3,3.9 \pm 0.3,4.8 \pm 0.3$, and 4.3 \pm 0.4 , respectively. To make sure that this decrease in $k_{\text {app }}$ is only due to deprotonation process, the same kind of experiments were studied at PYR/Pt electrodes and the result is shown in Figure 6. Constant $k_{a p p}$ values of these non-acid-terminated monolayers confirm the previous conclusion. These $\mathrm{pK}_{\mathrm{a}}$ values are slightly higher than that of the corresponding precursors in water except PPA/Pt. The decrease in $\mathrm{pK}_{\mathrm{a}}$ for PPA/Pt is most likely due to the electron withdrawing effect of the aromatic ring when coupled to Pt.

\section{Determination of $\mathbf{p K a}$ using IRRAS}

The IRRAS spectra in Figure 11 are the low energy spectra of INA/Pt as a function of solution $\mathrm{pH}$. Obviously, the peak intensity of $v(\mathrm{C}=\mathrm{O})$ decreases with increasing solution $\mathrm{pH}$ while that of $v_{\mathrm{s}}(\mathrm{COO}-)$ peak at $1389 \mathrm{~cm}^{-1}$ and the $\nu_{\mathrm{a}}(\mathrm{COO}-)$ at $1520 \mathrm{~cm}^{-1}$ increase with increase in solution $\mathrm{pH}$. Notice that the $\mathrm{v}(\mathrm{C}-\mathrm{O})$ at $1181 \mathrm{~cm}^{-1}$ decreases with increase in solution $\mathrm{pH}$.

The complete disappearance of the peak at $1181 \mathrm{~cm}^{-1}$ at high solution $\mathrm{pH}$ proves that this peak is the $v(\mathrm{C}-\mathrm{O})$ mode only without anything else as cited in literature ${ }^{30}$.

The peak areas of $v(\mathrm{C}=0), v_{\mathrm{s}}(\mathrm{COO}-)$, and $v(\mathrm{C}-\mathrm{O})$ are shown in Figure 12. The peak area of non-hydrogen-bonded $v(\mathrm{C}=0)$ starts to decrease at very low $\mathrm{pH}$ and has a transition of $\sim 3 \mathrm{pH}$ units. The peak area of the hydrogen-bonded dimeric $\mathrm{C}=\mathrm{O}$ starts to decrease at $\mathrm{pH} \sim 3.5$ with a transition range of $6 \mathrm{pH}$ units. The $\mathrm{pK}_{\mathrm{a}}$ values calculated from the reflection 


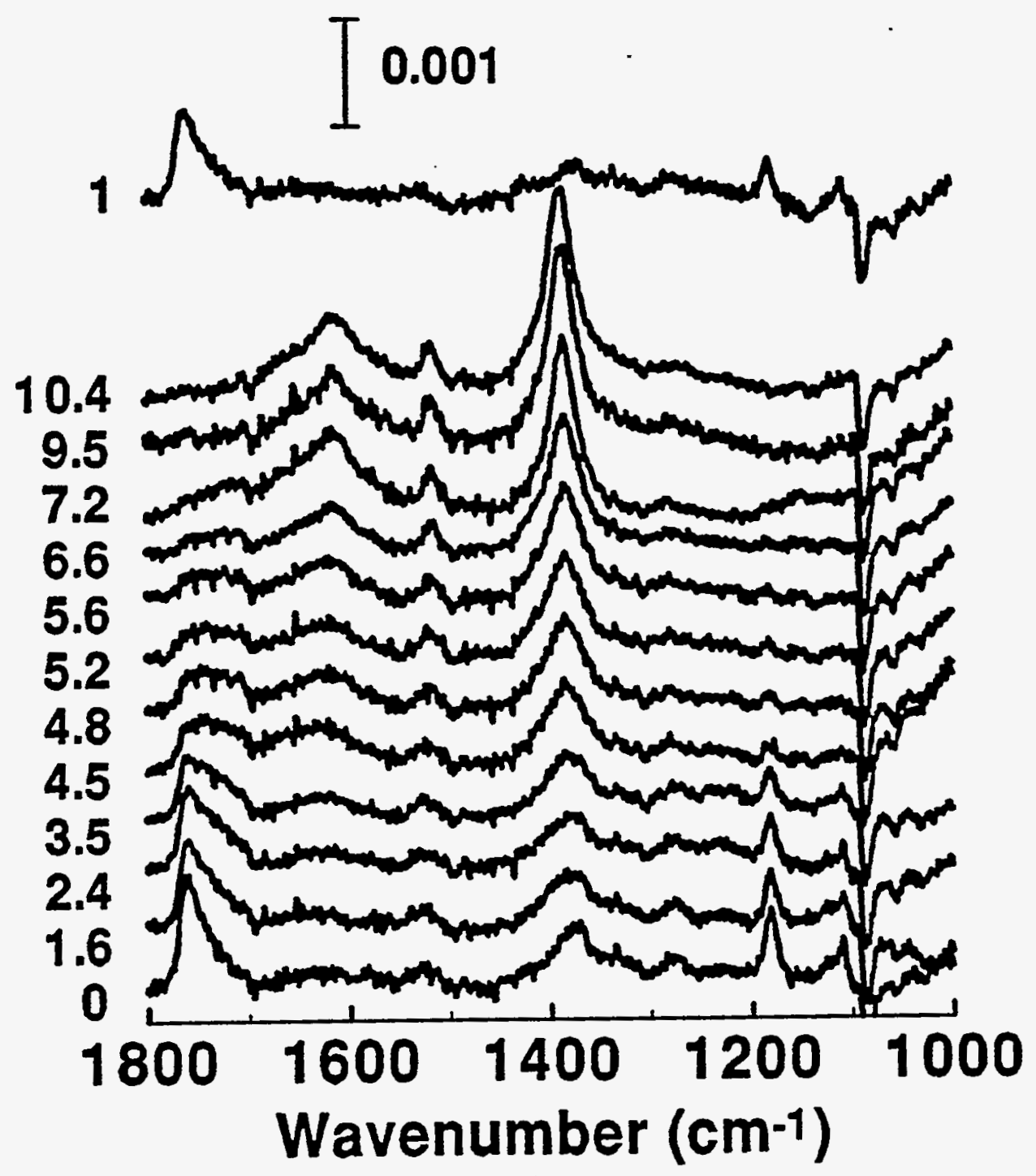

Figure 11. IRRAS spectra in the low energy region for INA/Pt at $80^{\circ}$ degree of incident angle from the surface as a function of solution $\mathrm{pH}$. 


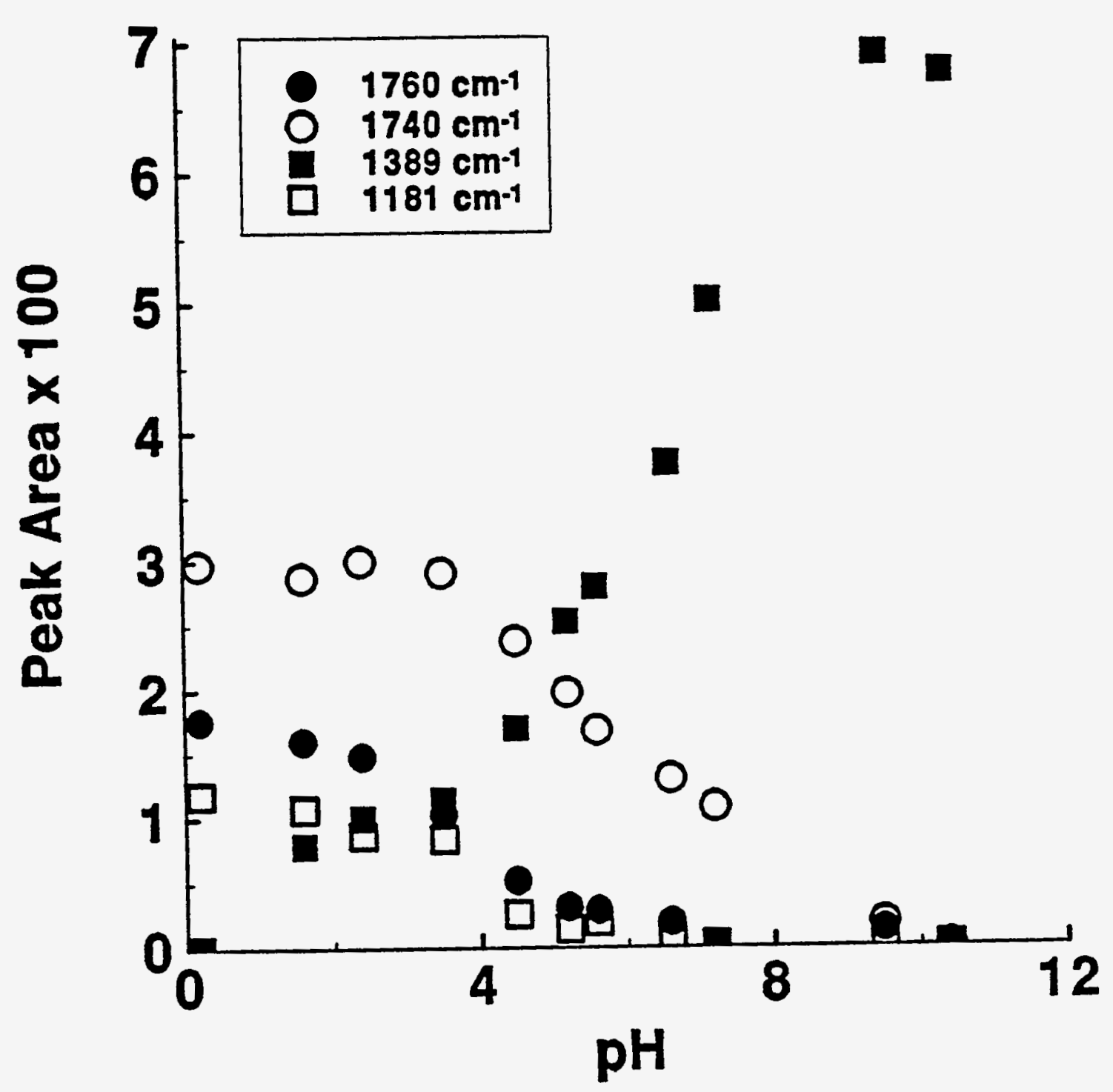

Figure 12. The IRRAS peaks at $1760 \mathrm{~cm}^{-1}, 1740 \mathrm{~cm}^{-1}, 1389 \mathrm{~cm}^{-1}$, and $1181 \mathrm{~cm}^{-1}$ of INA/Pt at $80^{\circ}$ degree of incident angle from the surface as a function of solution $\mathrm{pH}$. 
point of the curves for non-hydrogen-bonded and hydrogen-bonded dimeric $\mathrm{C}=\mathrm{O}$ groups are 3.8 and 6.0 , respectively. The $\mathrm{pK}_{\mathrm{a}}$ value of non-hydrogenbonded $\mathrm{C}=\mathrm{O}$ is almost the same as that of the adsorbate precursor in water $(3.44)^{56}$. On the other hand, the $\mathrm{pK}_{\mathrm{a}}$ of hydrogen-bonded dimeric $\mathrm{C}=\mathrm{O}$ group is higher than that of the adsorbate precursor in water by $2.56 \mathrm{pH}$ unites. The calculated $\mathrm{pK}_{\mathrm{a}}$ value for NA/Pt is listed in Table 4 for comparison as well.

Interestingly, the change in the peak area of $v(\mathrm{C}-\mathrm{O})$ not only has a reflection point the same as the $\mathrm{pK}_{\mathrm{a}}$ of non-hydrogen-bonded $\mathrm{C}=\mathrm{O}$ group but also has the same trend as non-hydrogen-bonded $\mathrm{C}=\mathrm{O}$ group. This suggests that the $v(\mathrm{C}-\mathrm{O})$ mode observed at $1181 \mathrm{~cm}^{-1}$ is associated with non-hydrogen-bonded $\mathrm{C}=\mathrm{O}$ group rather the hydrogen-bonded dimeric $\mathrm{C}=\mathrm{O}$ group. This implies that the spatial orientation of non-hydrogen-bonded $\mathrm{C}=\mathrm{O}$ group is quite different from that of hydrogen-bonded dimeric $\mathrm{C}=\mathrm{O}$ group. It is strange that there is not much of the amount of COO- until $\mathrm{pH}$ 4. In fact the peak area of the $\mathrm{COO}$ - increases from $\sim 0.01$ at $\mathrm{pH} 4$ to $\sim 0.07$ at $\mathrm{pH} 10$. This suggests that the orientation of the $v_{\mathrm{s}}(\mathrm{COO}-)$ dipole from the non-hydrogen-bonded $\mathrm{C}=\mathrm{O}$ group is more parallel to the $\mathrm{Pt}$ surface than that from the hydrogen-bonded diemric $\mathrm{C}=\mathrm{O}$ group.

\section{Determination of $\mathrm{pKa}$ using contact angle measurements}

Contact angle measurements have long been used to probe wettability". Recently, this method was used to "titrate" acidity of polymers $^{58}$ and carboxylic acid monolayers ${ }^{59}$ structure and metal oxides ${ }^{60}$. The basis of a contact angle measurement is based on the Young equation ${ }^{61}$.

$$
\gamma_{\mathrm{SV}}-\gamma_{\mathrm{SL}}=\gamma_{\mathrm{LV}} \cos \theta
$$

where $\gamma_{S V}$ and $\gamma_{L V}$ are the respective solid and liquid surface tensions in equilibrium with the saturated liquid vapor, $\gamma_{\mathrm{SL}}$ is the surface tension between solid and liquid, and $\theta$ is the contact angle between liquid and solid. Since changes in both $\gamma_{S V}$ and $\gamma_{\mathrm{LV}}$ with $\mathrm{pH}$ are relatively small, it follows that decreases in $\theta$ with $\mathrm{pH}$ reflect an increase in $\gamma_{\mathrm{SL}}{ }^{60}$. This is the 
Table 4. The $\mathrm{pK}_{\mathrm{a}}$ values of BA/Pt, PAA/Pt, PPA/Pt, PBA/Pt, INA/Pt, NA/Pt and PAA/Pt monolayers based on IRRAS, electrochemistry (ECHEM) and contact angle (CA) measurements.

\begin{tabular}{lcccc}
\hline $\mathrm{pK}_{\mathrm{a}}$ & IRRAS $^{\mathrm{a}}$ & ECHEM $^{\mathrm{a}}$ & $\mathrm{CA}^{\mathrm{a}}$ & $\begin{array}{c}\text { aqueous solution } \\
\text { value }\end{array}$ \\
\hline BA/Pt & b & none & $\mathrm{c}$ & 4.19 \\
PAA/Pt & b & none & c & 4.9 \\
PPA/Pt & b & 3.5 & $\mathrm{c}$ & 4.9 \\
PBA/Pt & b & 5.3 & $\mathrm{c}$ & 5.0 \\
INA/Pt & $3.8,6.0$ & 3.9 & 4.0 & $3.75^{\mathrm{d}}$ \\
NA/Pt & $3.9,6.0$ & 4.8 & 4.7 & $3.44^{\mathrm{d}}$ \\
PAA/Pt & b & 4.3 & 4.6 & $3.88^{\mathrm{d}}$ \\
\hline
\end{tabular}

${ }^{a}$ The standard deviation for IRRAS, ECHEM, and CA data are $0.3,0.3$, and $0.4 \mathrm{pK}$ units, respectively.

${ }^{b}$ No detectable spectroscopic signal.

${ }^{c}$ The contact angle at low $\mathrm{pH}$ is low for titration.

${ }^{\mathrm{d}}$ Refer to reference 56. 
basis of the "contact angle titration" measurement.

The following presents results from using this technique to characterize three of our reactive monolayers samples (i.e. NA/Pt, INA/Pt, and PAA/Pt) and a control sample (PYR/Pt); the low surface tension of other monolayers precludes this characterization. As with the IRRAS data, these measurements were performed for methods verification. The contact angles of water at NA/Pt, INA/Pt, PAA/Pt, and PYR/Pt as a function of $\mathrm{pH}$ are shown in Figures 13-15. As can been seen, the contact angles of water at NA/Pt, INA/Pt, and PAA/Pt decrease upon increases in $\mathrm{pH}$. This is due to progressive ionization of the carboxylic acid functionalities, which more and more amount of the carboxylate groups which have higher surface energy than that of carbonyl groups upon deprotonation ${ }^{58,59}$. As expected, PYR/Pt does not show any change as shown in Figure 16.

It is possible to calculate $\mathrm{pK}_{\mathrm{a}}$ of these acid on Pt surface based on the relationship between $\cos \theta_{\mathrm{a}}$ and solution $\mathrm{pH}$. The $\mathrm{pK}_{\mathrm{a}}$ values of $\mathrm{INA} / \mathrm{Pt}$, NA/Pt and PAA/Pt surface, which were calculated at $50 \%$ of the total change in $\cos \theta_{a}$, were found to be $4.0 \pm 0.4,4.7 \pm 0.4$, and $4.6 \pm 0.4$, respectively. (see Table 4 )

\section{Comparison of $\mathrm{pKa}$ values}

The $\mathrm{pK}_{\mathrm{a}}$ values of NA/Pt, INA/Pt and PAA/Pt have been calculated by using IRRAS, cyclic voltammetry and contact angle measurements and are listed in Table 4. Although the standard deviations of these values are a little large because of experiment difficulties, it is possible to make some comparisons from these values.

Owing to too weak IRRAS signals of PAA/Pt, there is no $\mathrm{pK}_{\mathrm{a}}$ value available for PAA/Pt from IRRAS data. The $\mathrm{pK}_{\mathrm{a}}$ values of pyridylcarboxylic acids in water are calculated values ${ }^{57}$ instead of the dissociation constants because these acids are zwitterionic. From IRRAS data, both NA/Pt and INA/Pt monolayers have two $\mathrm{pK}_{\mathrm{a}}$ values. The $\mathrm{pK}_{\mathrm{a}}$ values for non-hydrogen-bonded $\mathrm{C}=\mathrm{O}$ group are almost the same as that in water. On the other hand, the $\mathrm{pK}_{\mathrm{a}}$ values for dimeric hydrogen-bonded $\mathrm{C}=\mathrm{O}$ group for NA/Pt and INA/Pt are higher than that in water by $\sim 2.5 \mathrm{pH}$ 


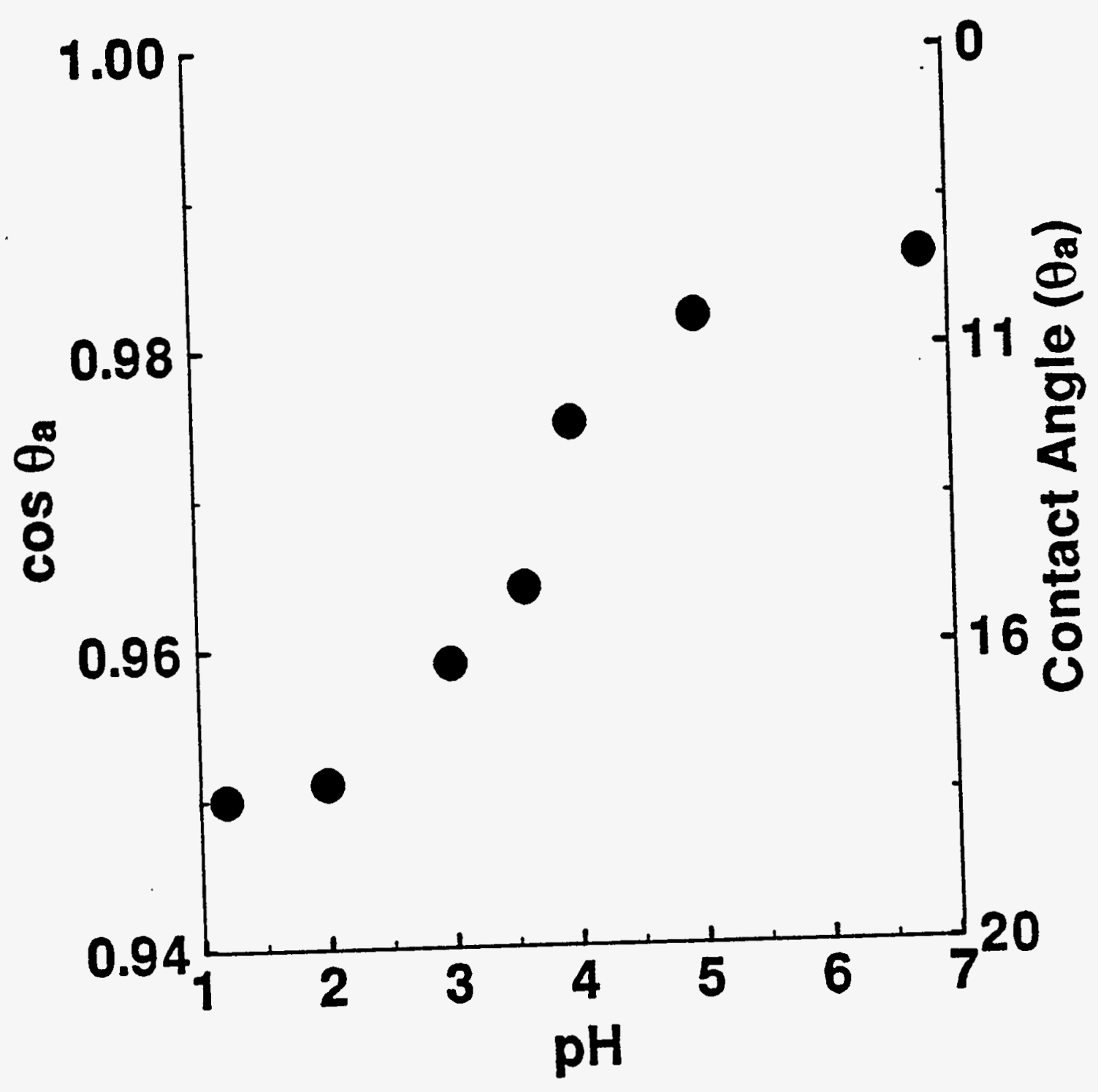

Figure 13. The $\cos \theta_{a}$ of water on the INA/Pt surface as a function of solution $\mathrm{pH}$. 


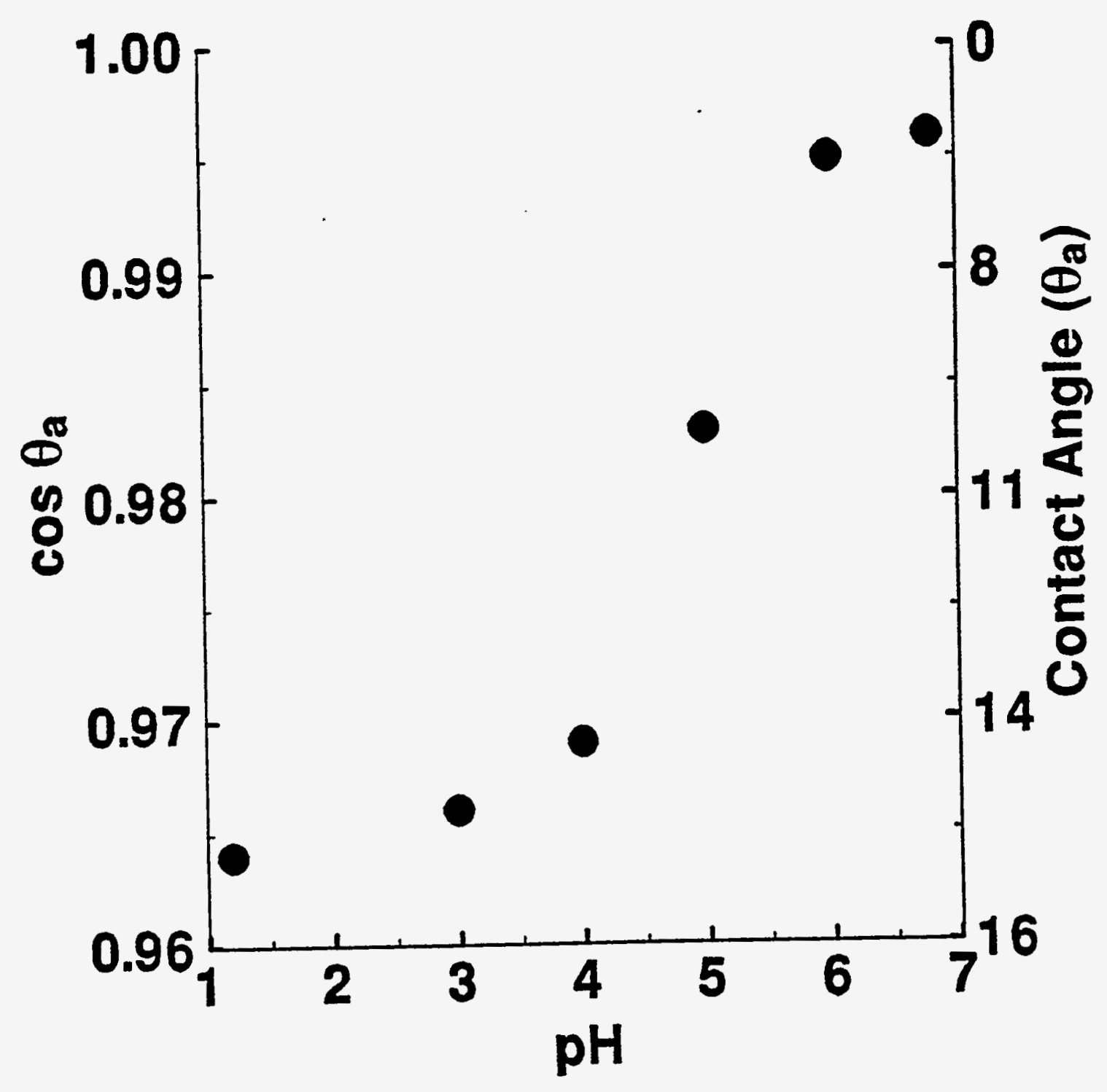

Figure 14. The $\cos \theta_{\mathrm{a}}$ of water on the NA/Pt as a function of solution $\mathrm{pH}$. 


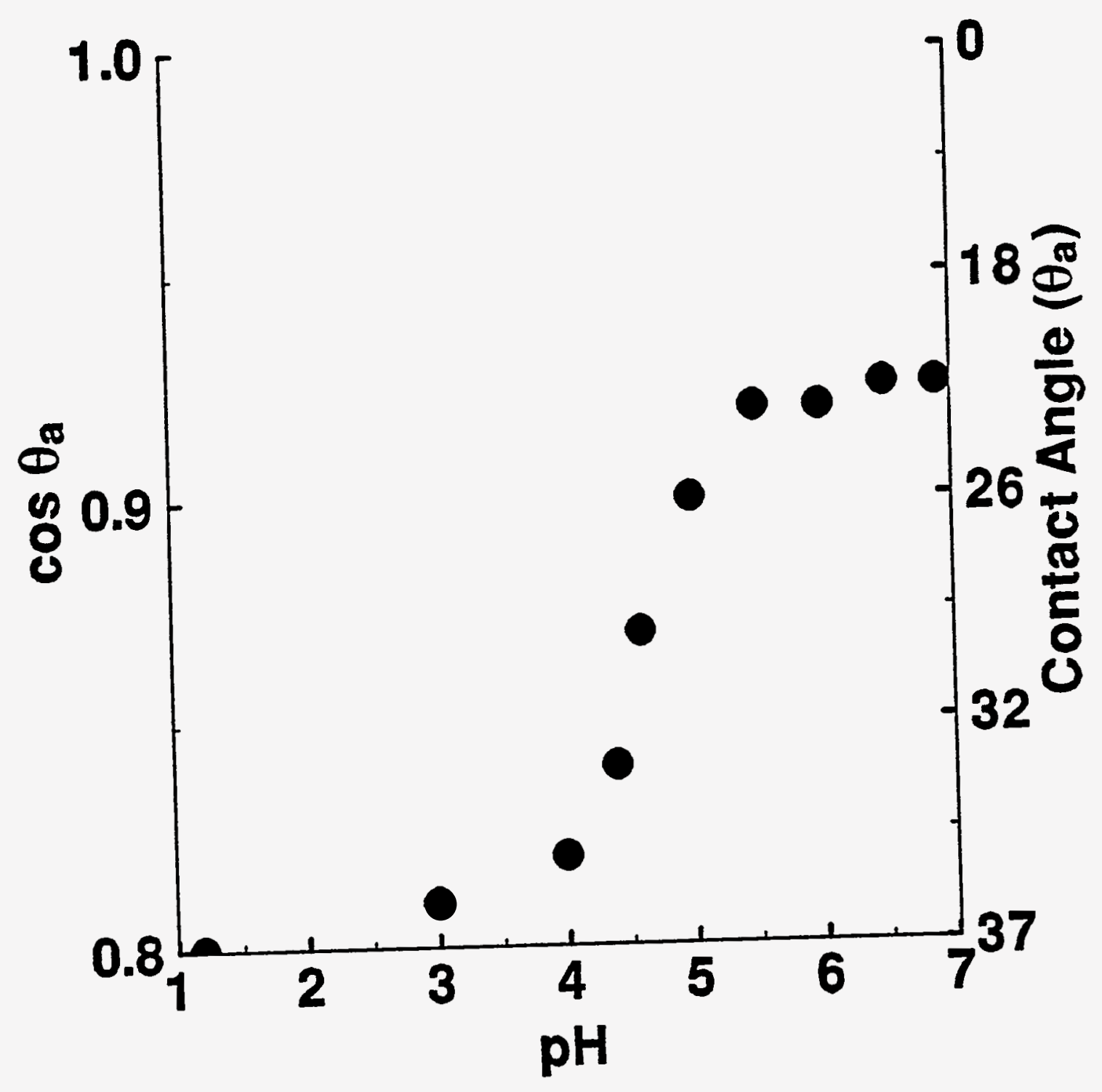

Figure 15. The $\cos \theta_{a}$ of water on the PYAA/Pt as a function of solution $\mathrm{pH}$. 


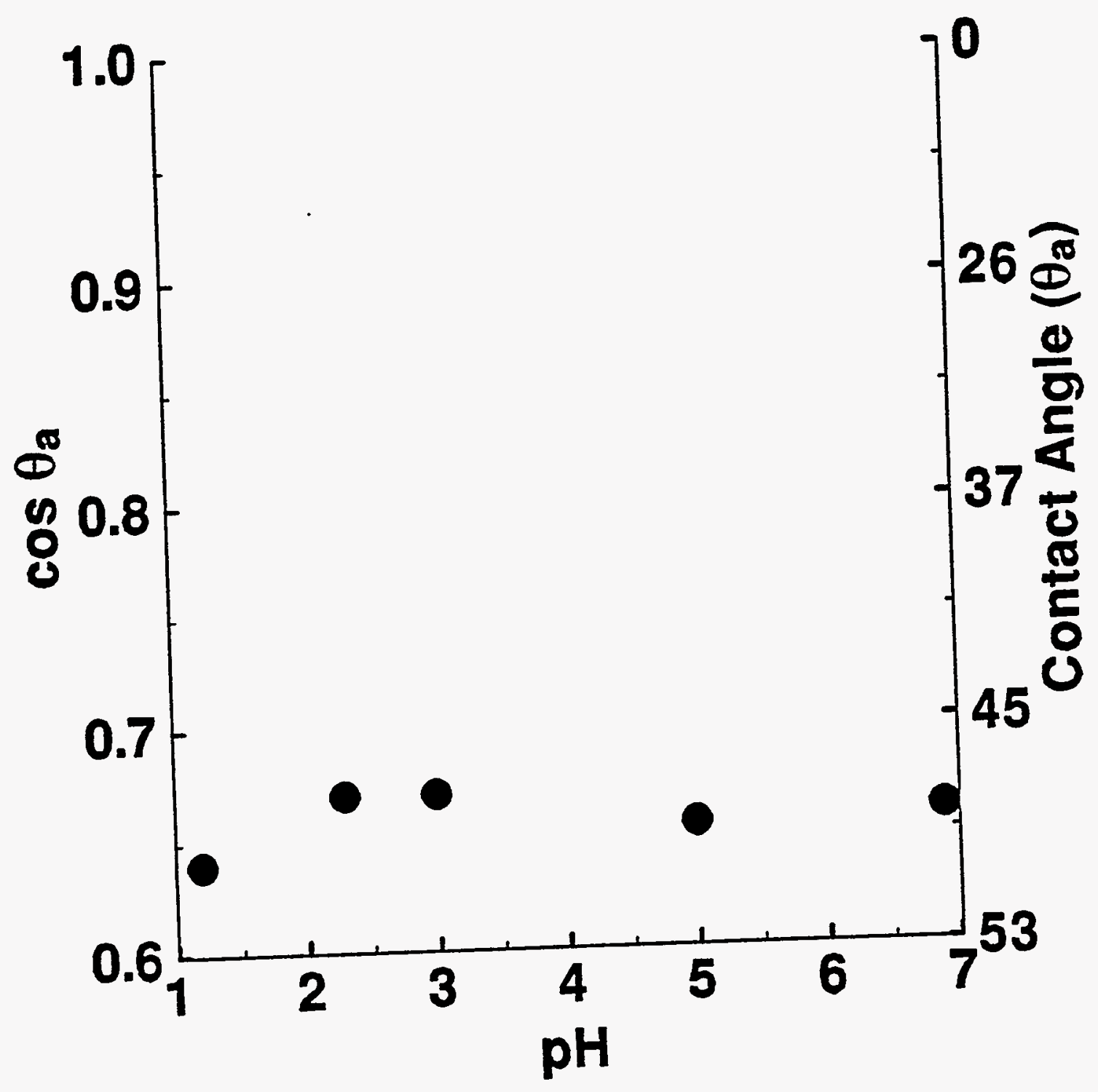

Figure 16. The $\cos \theta_{\mathrm{a}}$ of water on the PYR/Pt as a function of solution $\mathrm{pH}$. 
units. This strongly suggests that the decrease in the acidity of both INA/Pt and NA/Pt is primly due to the presence of the hydrogen bonding but not the low dielectric environment.

Both electrochemistry and contact angle measurements can only provide the overall acidity and give almost the same $\mathrm{pK}_{\mathrm{a}}$ values for INA/Pt, $\mathrm{NA} / \mathrm{Pt}$, and PAA/Pt. All these INA/Pt, NA/Pt, and PAA/Pt monolayers have higher $\mathrm{pK}_{\mathrm{a}}$ values than that of corresponding precursor in water by $\sim 0.2, \sim 1.3, \sim 0.6 \mathrm{pH}$ units, respectively. In other words, deprotonation process of these pyridylcarboxylic acid on Pt surfaces is more difficult than in water due to the existence of hydrogen bonding. 


\section{CONCLUSIONS}

Self-assembly monolayers of short methylene phenylcarboxylic acids on Pt can form the closest-packed structure with the rings and methylene groups oriented parallel to the $\mathrm{Pt}$ surface that can be exhaustively oxidized to $\mathrm{CO}_{2}$ and $\mathrm{H}_{2} \mathrm{O}$ electrochemically. Unlike phenylcarboxylic acids, pyridylcarboxylic acids are bonded to $\mathrm{Pt}$ surface through nitrogen rather the aromatic rings and have the ring oriented almost perpendicular to the surface. This makes pyridylcarboxylic acid monolayers on Pt more difficult to be oxidized electrochemically than aromatic acid monolayers on Pt.

Deprotonation of all these acids on Pt electrodes causes $0 \%$ to $40 \%$ and $85 \%$ decrease in the heterogeneous electron transfer rate of ferricyanide at phenylcarboxylic acids coated Pt electrodes and pyridylcarboxylic acids coated Pt electrodes, respectively. The changes in the potential at the reaction plane are about $0 \mathrm{mV}$ to $-5 \mathrm{mV}$ and $-20 \mathrm{mV}$ for phenylcarboxylic acid coated Pt electrodes and pyridylcarboxylic acids coated Pt electrodes, respectively. The observed $\Delta \phi_{\mathrm{S}}$ values due to deprotonation for PPA/Pt and PBA/Pt are only $0.7 \%$ of the theoretical values and that for INA/Pt and $\mathrm{NA} / \mathrm{Pt}$ are only $1.2 \%$ due to the screening effect of the electrolyte and water. No change in $\phi_{\mathrm{T}}$ was observed for BA/Pt and PAA/Pt electrodes because both BA and PAA lose the carboxylic acid groups upon immobilization onto Pt surfaces.

INA, NA, PYAA become weaker acids mainly because of the formation of hydrogen bond while PPA becomes a stronger acid because of the electron withdrawing nature of the aromatic ring. 


\section{REFERENCES}

(1) Kajzar, F.; Messier, J.; Zyss, J.; Ledoux, I. Opt. Commun., 1983, 45, 13.

(2) Carter, G. M.; Chen, Y. J.; Tripathy, S. K. Opt. Engl. 1985, 24, 609.

(3) Mathur, S.C.; Scheinbeim, J. I.; Newman, B. A. J. Appl. Phys. 1984, 56, 2419.

(4) Murray, R. W. Annu. Rev. Mater. Sci. 1984, 14, 145.

(5) Stern, D. A.; Wellner, E.; Salaita, G. N.; Laguren-Davidson, L.; Lu. F.; Batina, N. Frank, D. G.; Zapien, D. C.; Walton, N.; Hubbard, A. T. J. Am. Chem. Soc. 1988, 110, 4885.

(6) Soriaga, M. P.; Hubbard, A. T. J. Am. Chem. Soc. 1982, 104, 2735.

(7) Soriaga, M. P.; Wilson, P. H.; Hubbard, A. T.; Benton, C. S. J. Electroanal. Chem. 1982, 142, 317.

(8) Soriaga, M. P.; Hubbard, A. T. J. Am. Chem. Soc. 1982, 104, 3937.

(9) Hubbare, A. T.; Stickney, J. L.; Soriaga, M. P.; Chia, V. K. F.; Rosasco, S. D.; Schardt, B. C.; Solomun, T.; Song, D.; White, J. H.; Wieckowski, A. J. Electroanal. Chem. 1984, 168, 43.

(10) Lai, C, N.; Hubbard, A. T. Inorg. Chem. 1972, 11, 2081.

(11) Ishikawa, R. M.; Katekaru, J.; Hubbard, A. T. J. Electroanal. Chem. 1978, 86, 271.

(12) Katekaru, J.; Hershberger, J.; Garwood, G. A. Jr.; Hubbard, A. T. Surface Sci. 1982, 121, L524.

(13) Lumsden, J. B.; Garwood, G. A.; Hubbard, A. T. J. Am. Chem. Soc. $1982,104,3937$.

(14) Soriaga, M. P.; Hubbard, A. T. J. Am. Chem. Soc. 1982, 104, 2742.

(15) Gui, J. Y.; Kahn, B. E.; Laguren-Davidson, L.; Lin, C.-H.; Lu, F.; Salaita, G. N.; Stern, D. A.; Hubbard, A. T. Langmuir 1989, 5, 819.

(16) White, J. H.; Soriaga, M. P.; Hubbard, A. T. J. Electroanal. Chem. 1984, 177, 89.

(17) Soriaga, M. P.; Stickney, J. L.; Hubbard, A. T. J. Electroanal. Chem. 1983, 144, 207.

(18) Stern, D. A.; Salaita, G. N.; Lu, F.; McCargar, J. W.; Batina, N.; Frank, D. G.; Laguren-Davidson, L.; Lin, C.-H.; Walton, N.; Gui, J. Y.; Hubbard, A. T. Langmuir 1988, 4, 711. 
(19) Soriaga, M. P.; Hubbard, A. T. J. Electroanal. Chem. 1984, 167, 79.

(20) Sasaki, K.; Kunai, A.; Harada, J.; Nakaboni, S. Electrochim. Acta, 1983, 28, 671.

(21) Vasiliev, Yu. B.; Bagotzky, V. S.; Khazova, O. A.; Krasnova, N. N.; Sergeeva, T. A.; Bogdanovich, V. B. Electrochim. Acta 1981, 26, 545.

(22) Vasiliev, Yu. B.; Bagotzky, V. S.; Khazova, Cherny, V. V.; Meretsky, L. M. J. Electroanal. Chem. 1979, 98, 253.

(23) Mirkind, L. A.; Fioshin, M. Y.; Dubinin, A. G. Elektrokhimiya 1973, 9, 257.

(24) Mirkind, L. A.; Kazarinov, A. N.; Dubinin, A. G.: Fioshin, M. Y. Elektrokhimiya 1973, 9, 1067.

(25) Mirkind, L. A.; Kazarinov, A. N.; Dubinin, A. G.: Fioshin, M. Y. Elektrokhimiya 1973, 9, 1069.

(26) Gileadi, E.; Duic, L.; Bockris, J.O'M. Electrochim. Acta 1968, 13, 1915.

(27) Bockris, J.O'M.; Green, M.; Swinkels, D. A. J. J. Electrochem. Soc. 1964, 111, 743.

(28) Horanyi, G.; Nagy, E. J. Electroanal. Chem. 1973, 43, 441.

(29) Horanyi, G.; Andreev, V. N. J. Electroanal. Chem. 1982, 18, 1209.

(30) Stern, D. A.; Laguren-Davidson, Laarni; Frank, D. G.; Gui, J. Y.; Lin, C.-H.; Lu, F.; Salaita, G. N.;Walton, N.; Zapien, D. C.; Hubbard, A. T. J. Am. Chem. Soc. 1989, 111, 877.

(31) Fawcett, W. R. J. Chem. Phys. 1974, 61, 3842.

(32) Parson, R. J. Electroanal. Chem. 1969, 21, 35.

(33) Nicolson, R. S. Anal. Chem., 1965, 37(11), 667.

(34) Heyrovsky, J.; Kuta, J. Principle of Polarography, Academic Press, New York, 1966, p. 106.

(35) Nicolson, R. S. Anal. Chem., 1964, 37, 1351.

(36) Perone, S. P. Anal. Chem., 1966, 38, 1158.

(37) Stickney, J. L.; Soriaga, M. P.; Hubbard, A. T.; Anderson, S. E. J. Electroanal. Chem. 1981, 125, 73.

(38) Pauling, L. C. The Nature of the Chemical Bond, 3rd ed.; New York: Cornell University Press, 1960. 
(39) Lane, R. F.; Hubbard, A. T. J. Phys. Chem. 1973, 77, 1411.

(40) Frumkin, A. N. Z. Physik. Chem. 1933, 164, 121.

(41) Frumkin, A. N.; Florianovich, G. M. Zh. Fiz. Khim. 1955, 29, 1827.

(42) Frumkin, A. N. Z. Elektrochem. 1955, 59, 807.

(43) Frumkin, A. N. Advan. Electrochem. Eng. 1961, 1, 65.

(44) Parsons, R. Advan. Electrochem. Eng. 1961, 1, 1.

(45) Grahame, D. C. J. Electrochem. Soc. 1951, 98, 343.

(46) Grahame, D. C. J. Am. Chem. Soc. 1958, 80, 4201.

(47) Grahame, D. C.; Parsons, R. J. Am. Chem. Soc. 1961, 83, 1291.

(48) Lawrence, J.; Parsons, R.; Payne, R. J. Electroanal. Chem. 1968, 16, 193.

(49) Schimid, R. W.; Reilley, C. N. J. Am. Chem. Soc. 1958, 80, 2087.

(50) Deakin, M. R.; Stutts, K. J.; Wightman, R. M. J. Electroanal. Chem. 1985, $182,113$.

(51) Weaver, M. J.; Satterberg, T. L. J. Phys. Chem. 1977, 81, 1772.

(52) Hu, I.-F.; Karweik, D. H.; Kuwana, T. J. Electroanal. Chem. 1985, $188,59$.

(53) Scherer, G.; Willig, F. J. Electroanal. Chem. 1977, 85,77.

(54) Randin, J. P.; Yeager, E. J. Electroanal. Chem. 1975, 58, 313.

(55) Bard, A. J.; Faulkner, L. R. Electrochemical Methods Fundamentals and Application, New York: Wiley, 1980; pp513-516.

(56) Blanch, J. H. J. Chem. Soc.(B), 1966, 937.

(57) Kaelble, D. H. Physical Chemistry of Adhesion, New York: Wiley, 1971.

(58) Holmes-Farlsy, S. R.; Whitesides, G. M. Langmuir, 1987, 3, 62.

(59) Bain, C. D.; Whitesides, G. M. Langmuir 1989, 5, 1370.

(60) Chau, L.-K.; Porter, M. D. J. Colloid and Interface Sci. 1991, 145, 283.

(61) Adamson, A. W. Physical Chemistry of Surface, 4th ed.; Wiley: New York, 1982, p338; 
PAPER II

INFRARED REFLECTION ABSORPTION SPECTROSCOPIC STUDY OF THE TEMPORAL EVOLUTION OF THE STRUCTURE OF ALKANE THIOLATE MONOLAYERS ON GOLD 


\section{INTRODUCTION}

The high stability, well-ordered, densely-packed structure, simple fabrication process, and very flexible modification of the self-assembled monolayers on gold have attracted worldwide attention. As a consequence, the study of the properties and construction of the self-assembled thiol monolayers on gold has grown enormously in recent years ${ }^{1-3}$.

Porter and co-workers used infrared reflection absorption spectroscopy (IRRAS), ellipsometry and contact angle measurements to demonstrate that the tilt angle is $30^{\circ}$ with twist angle of $50^{\circ}$ and there is a odd-even chain-lengthdependent orientation of the methyl group which provides the best evidence of a conserved Au-S-C bond angle of the thiolate on Au monolayers ${ }^{4-6}$. Monolayers have a well ordered pattern of $\sqrt{3} \times \sqrt{3} \mathrm{R} 30^{\circ}$ with $5.0 \AA$ separation to the nearest neighbor from STM images ${ }^{7,8}$. The packing density calculated from the electrochemical reduction desorption data of these is $8.0 \mathrm{x}$ $10^{-10} \mathrm{~mol} / \mathrm{cm}^{2}$ on $\mathrm{Au} /$ mica annealed at $300^{\circ} \mathrm{C}$ assuming roughness factor of $1.2^{9}$. This supports the $\sqrt{3} \times \sqrt{3} \mathrm{R} 30^{\circ}$ structure with $30^{\circ}$ tilt angle from the surface normal. The loss of the mercapto hydrogen and formation of a gold alkanethiolate was concluded ${ }^{9}$.

Nuzzo and co-workers have used low-energy electron diffraction (LEED) and concluded that these monolayers have a $\sqrt{3} \times \sqrt{3} \mathrm{R} 30^{\circ}$ with a $4.99 \AA$ value for the distance between the nearest neighbor structure ${ }^{10}$. These molecular architecture is adopted independent of the chain length or chainterminating group and the tilt angle is $\sim 30^{\circ}$ with twist angle of $52^{\circ}$ which implies that the gold surface-sulfur-carbon bond angle is near $120^{\circ} .11$

Whitesides and co-worker have shown that the distance between the nearest neighbor is $4.97 \pm 0.05 \AA$ by transmission electron diffraction spectroscopy ${ }^{12}$. Bain and Whitesides have studied the wetting properties of a series of ethers of the form $\mathrm{HS}\left(\mathrm{CH}_{2}\right)_{16} \mathrm{O}\left(\mathrm{CH}_{2}\right)_{n} \mathrm{CH}_{3}$, where $\mathrm{n}$ varied from 0 to 5. They found that when $\mathbf{n}$ was $>1$ the surface properties of these material were identical to those of the pure n-alkane thiol monolayers ${ }^{13}$. This has demonstrated that these monolayers are sufficiently densely packed so that the 
surface properties measured are only those of the top layer but not those of the underlying polymethylene chains.

Pemberton and co-worker have used surface Raman scattering spectroscopy (SERS) and shown that the $\mathrm{H}$ in $\mathrm{H}-\mathrm{S}$ bond is gone when the molecule adsorbed, the C-S bond is largely parallel to the surface at $\mathrm{Au}$. The $\mathrm{S}-\mathrm{C}$ bond at $630 \mathrm{~cm}^{-1}$ is gauche mode, $700 \mathrm{~cm}^{-1}$ is trans mode. Gauche mode decreases with immersion time but small amount of gauche still exists due to defects in the film at grain boundaries on the polycrystalline Au surface. They calculated the tilt angle of $30^{\circ}$ and twist angle of ca. $45^{\circ} .^{14}$

Grunze and co-workers have calculated the tilt angle of these monolayers is $\sim 35^{\circ}$ by second harmonic generation spectroscopy ${ }^{15}$. and nearedge X-ray absorption fine structure spectroscopy (NEXAFS) ${ }^{16,17}$.

Shimazu and co-workers have used the electrochemical quartz micro balance and demonstrated that the number of adsorbed thiol molecules, $2.6 \mathrm{x}$ $10^{14}$ molecules $/ \mathrm{cm}^{2}$, estimated from the frequency change, suggests the formation of a monolayer of this thiol. This number agrees well with that calculated from the electrochemical charge associated with oxidation/reduction of the monolayer, $2.4 \times 10^{14}$ molecules $/ \mathrm{m}^{2}$ for ferrocenylundecanethiol ${ }^{18}$.

Klein and co-workers have used molecular dynamics calculations to demonstrate that these monolayers form a hugely corrugated yet dense surface phase with the chain cant toward the next-nearest neighbor ${ }^{19,20}$. The monolayers must be densely packed at $300^{\circ} \mathrm{K}$ with few gauche defects. The defects that exist are concentrated near the chain terminal ${ }^{21}$.

Ulman and co-workers have used molecular dynamics calculation and suggested that the tilt angle should be $30^{\circ}$ for n-alkane thiol monolayers on $\mathrm{Au}^{22}$.

Chidsey and co-workers have used helium diffraction technique and shown that the difference between the azimuths of maximum intensity for the ($1,-1)$ and $(n, 0)$ diffraction peaks is $30^{\circ}$, as would be expected for oriented domains of hexagonally packed adsorbates. The resulting lattice has a distance between the nearest neighbor of $5.01 \pm 0.02 \AA$. Shorter chains give the same lattice constant but weaker diffraction, and the domains appear to be much less well oriented. ${ }^{23}$. 
Although there are a few studies on the formation of these monolayers with a finding of a two-step processes including a Langmuirian kinetic model ${ }^{24-27}$, little work has been done concerning the details of this very fundamental adsorption process. In this study, the formation and the orientation of a 16-mercaptohexadecanoic acid $\left(\mathrm{HO}_{2} \mathrm{C}\left(\mathrm{CH}_{2}\right)_{15} \mathrm{SH}\right)$ and a n-octadecanethiol monolayers on $\mathrm{Au}$ were characterized using IRRAS as a function of immersion time. The discovery of agostic bonds at $2903 \mathrm{~cm}^{-1}$ due to the interaction between methylene groups and a bare Au surface based on the shift in energy of the $v_{a}\left(\mathrm{CH}_{2}\right)$ and the absence of the agostic bond at $2903 \mathrm{~cm}^{-1}$ on the $\mathrm{Au}$ surface which was pretreated with a sulfur monolayer is reported as well. 


\section{EXPERIMENTAL SECTION}

\section{Substrate preparation}

Electrodes were prepared by the resistive evaporation of $15 \mathrm{~nm}$ of chromium, followed by $300 \mathrm{~nm}$ of gold, onto 3 inch by 1 inch glass substrates. The temperature during the evaporation, measured at the plate supporting the substrates, increased to $\sim 50^{\circ} \mathrm{C}$ as a result of radiative heating by the evaporation source. The evaporation rates were 0.2 and $0.5 \mathrm{~nm} / \mathrm{s}$ for chromium and gold, respectively. The pressure in a cryopumped E360A Edwards Coating System during evaporation was $<9 \times 10^{-5} \mathrm{~Pa}$. After $\sim 45 \mathrm{~min}$ cooling time, the evaporator was back-filled with purified $\mathrm{N}_{2}$. The substrates were then removed, analyzed by optical ellipsometry for the determination of the optical functions of the uncoated substrates, and immersed in the thiolcontaining solution.

The surfaces of the uncoated gold electrodes have been characterized previously at both a macroscopic and microscopic level using scanning tunneling microscopy ${ }^{7}$ and electrochemical measurements of surface texture and surface area ${ }^{9}$. Macroscopically, the STM images show that the electrode surface is composed of atomically-terraced crystallites $\sim 25 \mathrm{~nm}$ in diameter. The electrodes have a roughness factor of $\sim 1.3$, which is given as the surface area determined from the oxidative desorption of iodine ${ }^{9}$ divided by the geometric electrode area.

\section{Monolayer preparation}

Thiolate monolayers were prepared by their spontaneous adsorption onto the evaporated gold from $1.00 \times 10^{-6} \mathrm{M}$ solutions in absolute ethanol for different periods of immersion time and rinsed successively with absolute ethanol and hexane, and dried under a stream of prepurified $\mathrm{N}_{2}$.

\section{Ellipsometric measurements of film thickness}

The thicknesses of the monolayers were determined by optical ellipsometry at $632.8 \mathrm{~nm}$ in two steps with a computer-interfaced Gaertner Model L-116B ellipsometer. The angle of incidence was $70^{\circ}$ from the surface normal. Upon removal from the evaporation chamber, the analyzer and 
polarizer angles for a reflected light beam from each uncoated substrate were measured on at least three different spots. The average complex refractive index for each substrate was then calculated with a two phase parallel layer model from classical electromagnetic theory ${ }^{28}$. After monolayer formation, each sample was again analyzed and the film thickness calculated from a threephase parallel layer model, using the average complex refractive index of the individual sample and a real refractive index of 1.45 for the film. A value of 1.45 , which is representative of the adsorbate precursors, facilitates comparison with thickness data that has been reported for a variety of monolayers. The influence of several refinements on this treatment of ellipsometric data have been recently discussed ${ }^{29}$.

\section{Infrared spectroscopy}

Infrared reflection absorption spectra (IRRAS) were acquired with a Nicolet 740 spectrometer. Spectra of the monolayers were obtained in a single reflection mode by using p-polarized light incident at $82^{\circ}$ from the surface normal and a liquid nitrogen cooled $\mathrm{HgCdTe}$ detector. A home-built sample holder was used to position the substrates reproducibly in the sample chamber of the spectrometer ${ }^{30}$. The spectrometer was purged with boil-off from liquid $\mathrm{N}_{2}$. Spectra were obtained by referencing 1024 sample scans to 1024 background scans at $2 \mathrm{~cm}^{-1}$ resolution (zero filled) with Happ-Genzel apodization. All spectra are reported as $-\log \left(R / R_{0}\right)$, where $R$ is the reflectance of the coated substrate and $R_{0}$ is that of a reference $A u$ substrate. The reference substrates were prepared by immersion of an uncoated gold substrate into $\sim 1 \mathrm{mM}$ ethanolic solution of octadecanethiol- $\mathrm{d}_{37}$.

for 24 hours.

\section{Chemicals and reagents}

The 16-mercaptohexadecanoic acid $\left(\mathrm{HO}_{2} \mathrm{C}\left(\mathrm{CH}_{2}\right)_{15} \mathrm{SH}\right)(\mathrm{MHA})$ was synthesized in this group and characterized with $\mathbb{R}$ and NMR. All other reagents including n-octadecanethiol (ODT) were obtained from Aldrich and used as received. Aqueous solutions were freshly prepared from water purified by passage through a Milli-Q purification system (Millipore Corp., Bedford, MA). 


\section{RESULTS AND DISCUSSION}

\section{IRRAS spectra as a function of immersion time}

MHA/Au

At low coverage, MHA/Au has only one observable $v_{a}\left(\mathrm{CH}_{2}\right)$ mode at $2903 \mathrm{~cm}^{-1}$ with no $v_{\mathrm{s}}\left(\mathrm{CH}_{2}\right)$ observed as shown in Figure 1. The absence of the $v_{\mathrm{a}}\left(\mathrm{CH}_{3, \mathrm{ip}}\right), v_{\mathrm{s}}\left(\mathrm{CH}_{3}, \mathrm{FR}_{1}\right)$, and $v_{\mathrm{s}}\left(\mathrm{CH}_{3}, \mathrm{FR}_{2}\right)$ modes at $2965 \mathrm{~cm}^{-1}, 2936 \mathrm{~cm}^{-1}$, and $\mathrm{cm}^{-1}$, respectively, confirms the formation of the acid-terminated monolayer. The absence of the $v_{\mathrm{s}}\left(\mathrm{CH}_{2}\right)$ and the presence of the $v_{\mathrm{a}}\left(\mathrm{CH}_{2}\right)$ strongly suggests that all the polymethylene groups must lie on the $\mathrm{Au}$ surface in order to have the $v_{\mathrm{s}}\left(\mathrm{CH}_{2}\right)$ dipole parallel to the Au surface and the $v_{\mathrm{a}}\left(\mathrm{CH}_{2}\right)$ perpendicular to the Au surface at the same time. The peak at $2903 \mathrm{~cm}^{-1}$ increases with increases in coverage and starts to decrease at high coverage where the $v_{\mathrm{a}}\left(\mathrm{CH}_{2}\right)$ and $v_{\mathrm{s}}\left(\mathrm{CH}_{2}\right)$ modes start to appear at $2926 \mathrm{~cm}^{-1}$ and 2860 $\mathrm{cm}^{-1}$, respectively. These two $r\left(\mathrm{CH}_{2}\right)$ peaks increase in peak area to a certain point where the peak at $2903 \mathrm{~cm}^{-1}$ totally disappear and decrease in peak area with decreases in peak frequency.

Figure 2 shows the IRRAS spectra in the low energy region of a MHA/Au as a function of immersion time. Peaks found at $1380 \mathrm{~cm}^{-1}, 1396$ $\mathrm{cm}^{-1}, 1436 \mathrm{~cm}^{-1}, 1460 \mathrm{~cm}^{-1}$ are $\omega\left(\mathrm{CH}_{2}\right), \delta\left(\alpha-\mathrm{CH}_{2}\right), v(\mathrm{C}-\mathrm{O})$, and $\delta\left(\mathrm{CH}_{2}\right)$, respectively. All the IRRAS modes assignment is listed in Table 1. The intensity of the $\delta\left(\alpha-\mathrm{CH}_{2}\right)$ and $v(\mathrm{C}-\mathrm{O})$ peaks decrease with increasing the coverage while that of $\omega\left(\mathrm{CH}_{2}\right)$ and $\delta\left(\mathrm{CH}_{2}\right)$ increase with increasing the coverage.

\section{ODT/Au}

The same trend was found in the case of ODT/Au as shown in Figures 3 and 4: it has only one observable $v_{a}\left(\mathrm{CH}_{2}\right)$ mode at $2903 \mathrm{~cm}^{-1}$ with no $v_{\mathrm{s}}\left(\mathrm{CH}_{2}\right)$ at low coverage and the intensity of the $\delta\left(\alpha-\mathrm{CH}_{2}\right)$ peak decreases with increasing the coverage while that of $\omega\left(\mathrm{CH}_{2}\right)$ increases with increasing the coverage.

In Figure 3, the peak at $2965 \mathrm{~cm}^{-1}, 2936 \mathrm{~cm}^{-1}$, and $2878 \mathrm{~cm}^{-1}$ are assigned as the $v_{\mathrm{a}}\left(\mathrm{CH}_{3, \mathrm{ip}}\right), v_{\mathrm{s}}\left(\mathrm{CH}_{3}, \mathrm{FR}_{1}\right)$, and $v_{\mathrm{s}}\left(\mathrm{CH}_{3}, \mathrm{FR}_{2}\right)$ modes, respectively. 


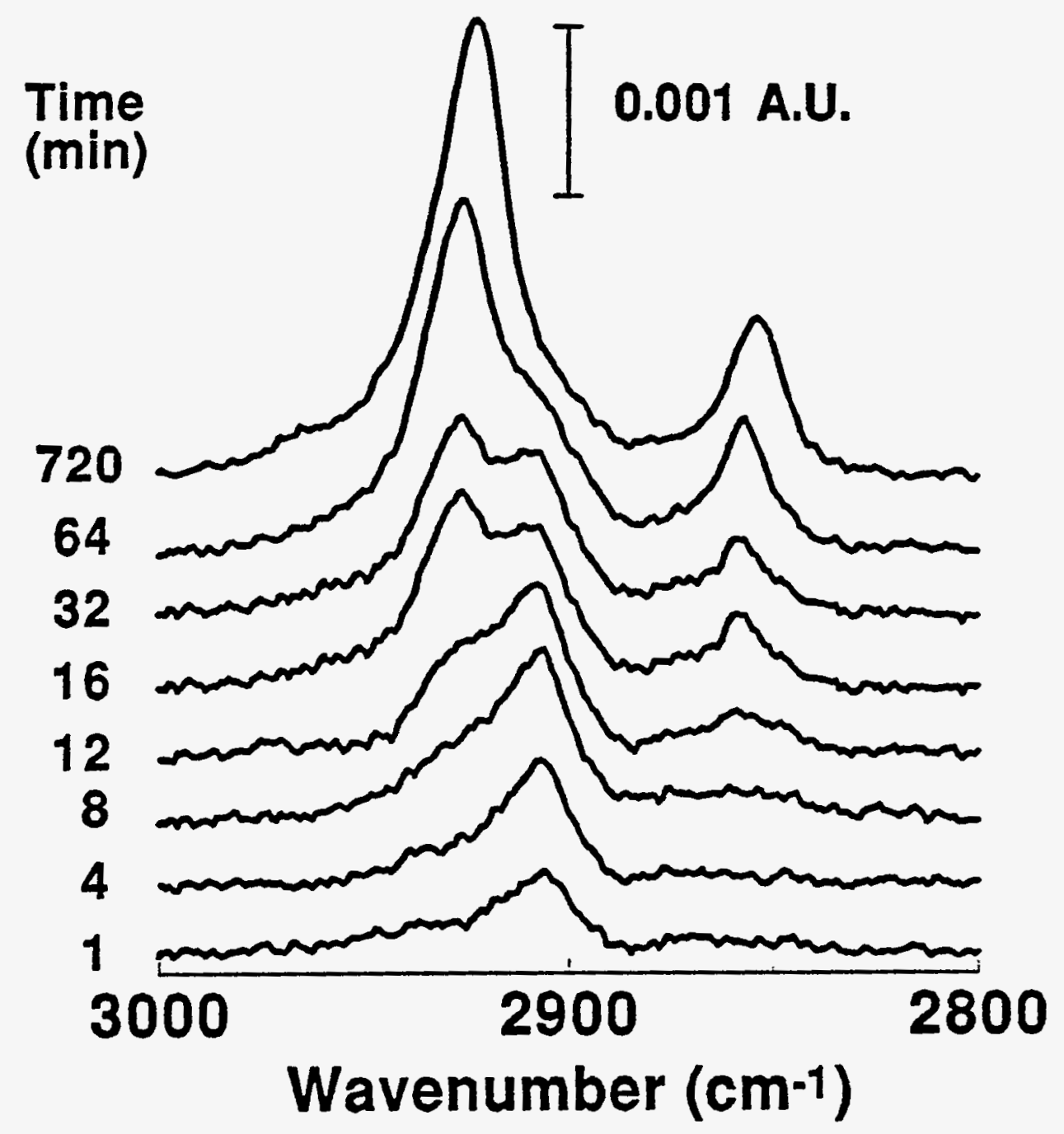

Figure 1. The IRRAS spectra in the high energy region of MHA on Au as a function of immersion time. 


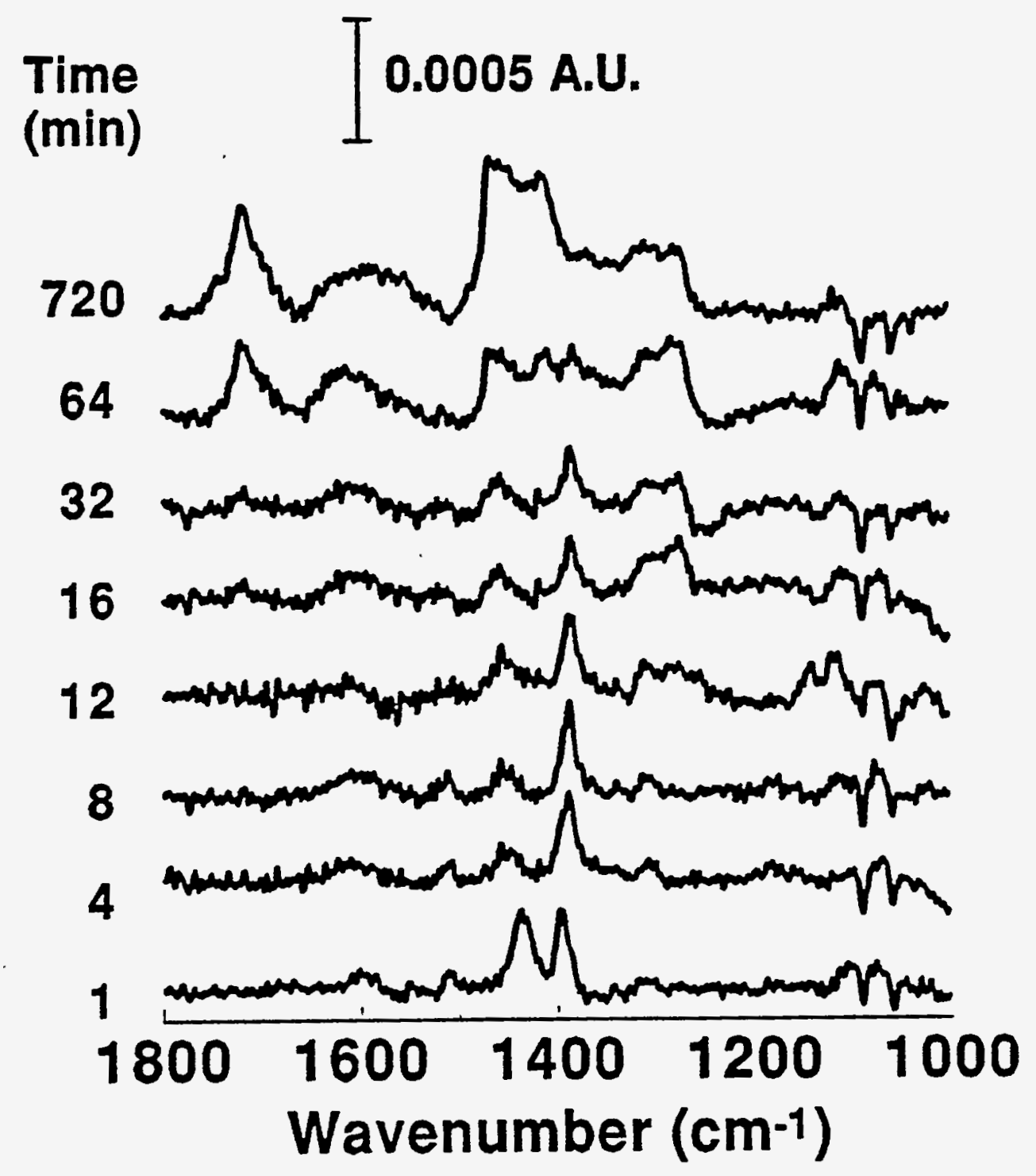

Figure 2. The IRRAS spectra in the low energy region of MHA on Au as a function of immersion time. 
Table 1. Peak positions, mode assignments, and transition dipole moment directions of carboxylic acid- and methyl-terminated alkanethiolate monolayers at gold.

$\mathrm{HO}_{2} \mathrm{C}\left(\mathrm{CH}_{2}\right)_{15} \mathrm{~S} / \mathrm{Au} \quad \mathrm{CH}_{3}\left(\mathrm{CH}_{2}\right)_{17} \mathrm{~S} / \mathrm{Au}$

direction of

Mode assignment obs. calc. obs. calc. transition dipole

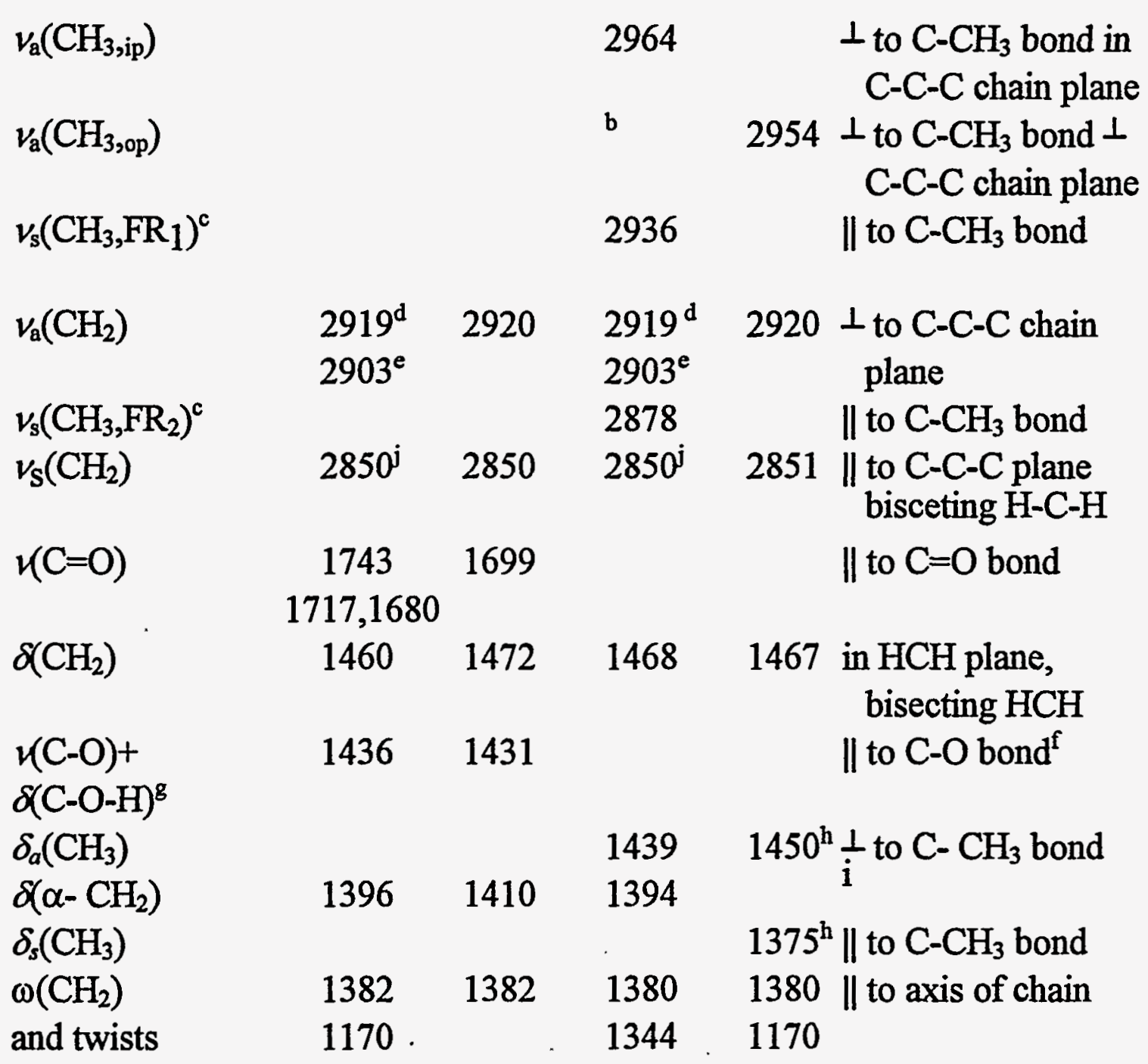


Table 1. (continued)

a Orientation of transition dipoles for $\mathrm{CH}$ modes assuming all-trans conformation (Snyder, R. G. J. Chem. Phys. 1965, 42, 1744). ' $v_{a}\left(\mathrm{CH}_{3, \mathrm{op}}\right)$ mode was not observed in the monolayer spectrum. ${ }^{\mathrm{C}} \mathrm{The}$ fermi-resonance couplet, $v_{\mathrm{S}}\left(\mathrm{CH}_{3}, \mathrm{FR}_{1}\right)$ and $v_{\mathrm{S}}\left(\mathrm{CH}_{3}, \mathrm{FR}_{2}\right)$, is designated by the subscripts 1 and 2, which refer to the higher and lower energy components, respectively. 'The peak frequency of this band shifts from $\sim 2926 \mathrm{~cm}^{-1}$ to $2919 \mathrm{~cm}^{-1}$ as increasing immersion time. ${ }^{e} T$ This $v_{\mathrm{a}}\left(\mathrm{CH}_{2}\right)$ is the agostic $\mathrm{C}-\mathrm{H}$...Au bond which has a direction of dipole perpendicular to the surface and will disappears when the coverage is high enough. ${ }^{f}$ The direction of the $v(\mathrm{C}-\mathrm{O})$ is more perpendicular to the surface at low coverage and more parallel to the surface at high coverage. ${ }^{8}$ This band involves both the stretching of the $\mathrm{C}-\mathrm{O}$ bond and the deformation of the C-O-H angle (Hadzi, D.and Pintar, M. Spectrochim. Acta, 1958, 12, 162.) ${ }^{h}$ Silverstein, R. M.; Clayton Bassler, G.; Morrill, T. C. Spectrometric Identification of Organic Compuonds 5th ed.; John Wiley \& Sons, Inc.: New York, 1991. 'The direction of this $\delta\left(\alpha-\mathrm{CH}_{2}\right)$ is more perpendicular to the surface at low coverage and more parallel to the surface at high coverage. ${ }^{j}$ The peak frequency of this band shifts from $\sim 2855 \mathrm{~cm}^{-1}$ to $2850 \mathrm{~cm}^{-1}$ as increasing immersion time. 


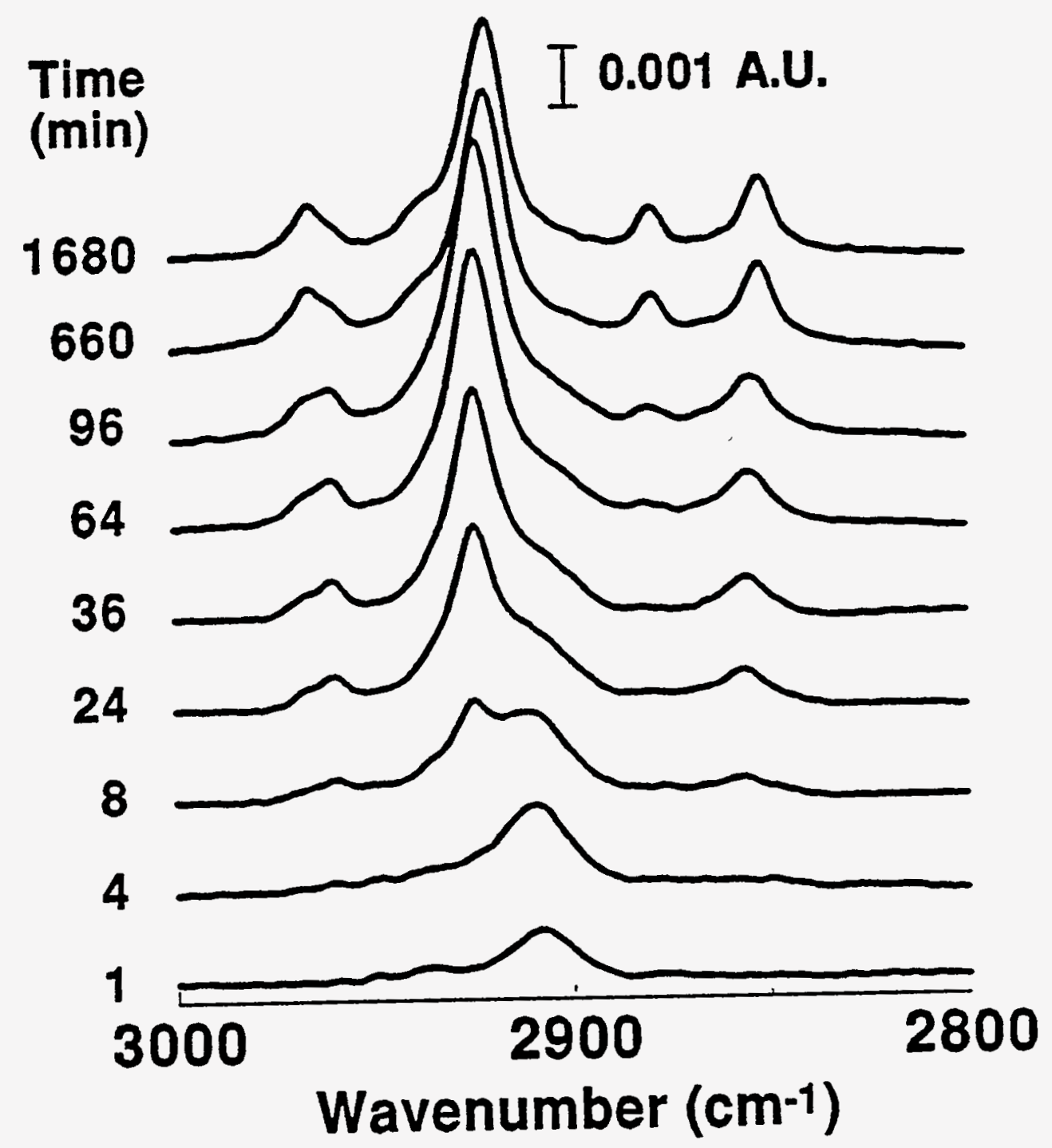

Figure 3. The IRRAS spectra in the high energy region of ODT on Au as a function of immersion time. 


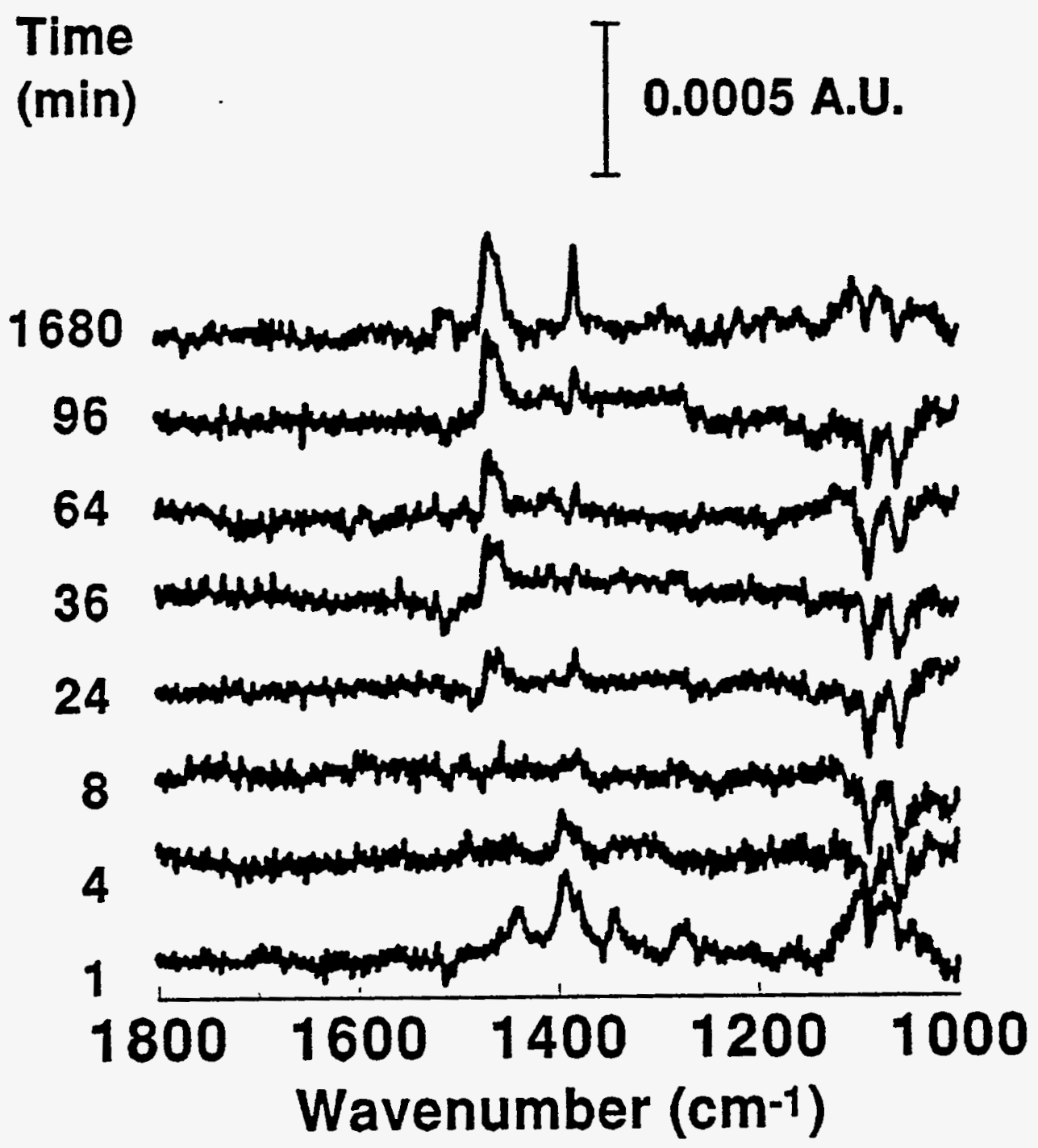

Figure 4. The IRRAS spectra in the low energy region of ODT on Au as a function of immersion time. 


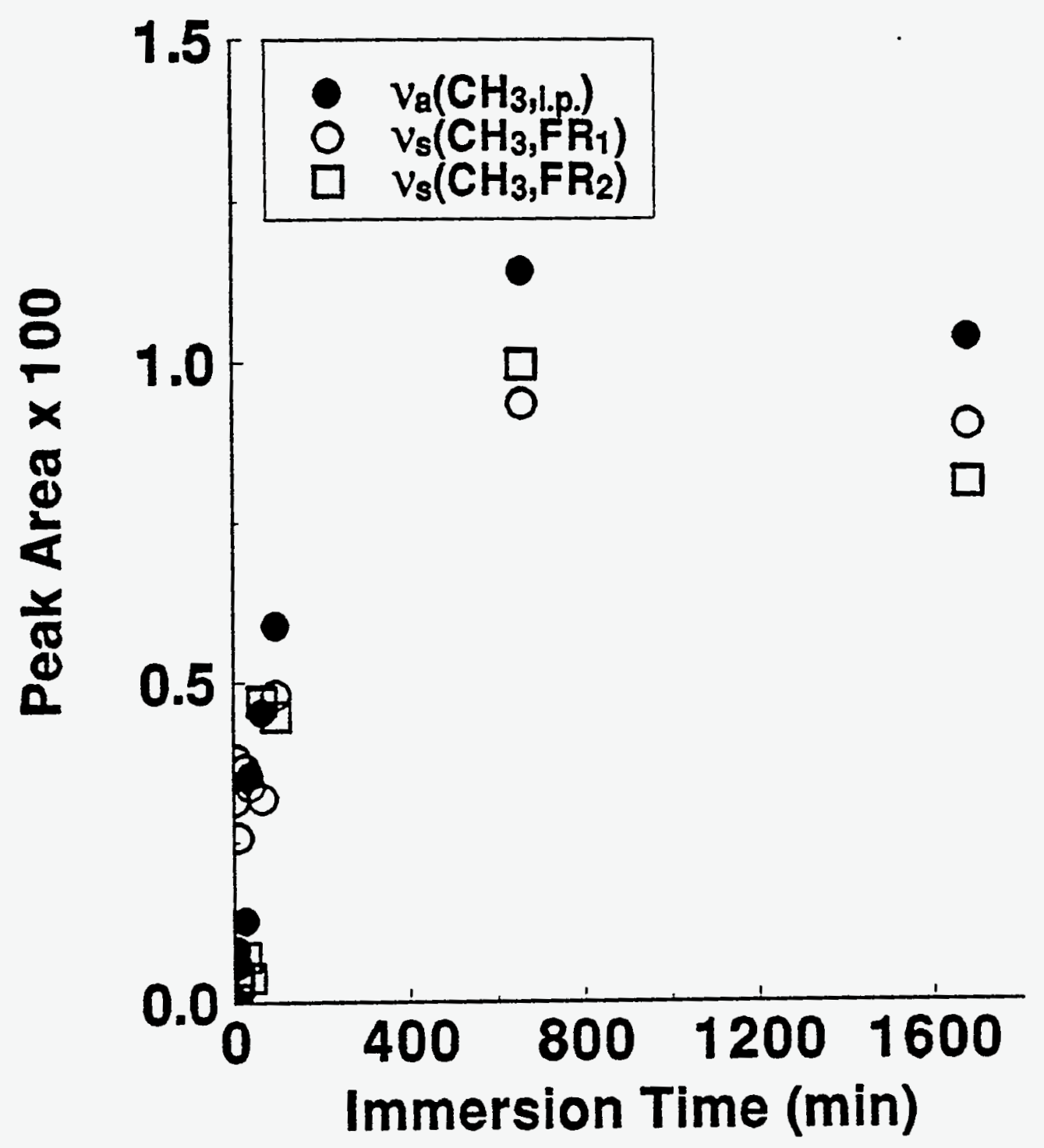

Figure 5. The peak area of $v_{\mathrm{a}}\left(\mathrm{CH}_{3}\right.$,ip $), v_{\mathrm{s}}\left(\mathrm{CH}_{3}, \mathrm{FR}_{1}\right)$, and $v_{\mathrm{s}}\left(\mathrm{CH}_{3}, \mathrm{FR}_{2}\right)$ of ODT on $\mathrm{Au}$ as a function of immersion time. 
The peak intensities of these three peaks increases along with the increase in that of the $v_{\mathrm{a}}\left(\mathrm{CH}_{2}\right)$ and $v_{\mathrm{s}}\left(\mathrm{CH}_{2}\right)$ modes at $2920 \mathrm{~cm}^{-1}$ and $2850 \mathrm{~cm}^{-1}$, respectively, while the peak intensity of the agostic $v_{\mathrm{a}}\left(\mathrm{CH}_{2}\right)$ at $2903 \mathrm{~cm}^{-1}$ decreases as shown in Figure 5. This strongly confirms that the polymethylene chain axis lies parallel to the surface at low coverage and stands up more and more as the coverage increases.

The absence of the $r(\mathrm{C}-\mathrm{O})$ peak at $1436 \mathrm{~cm}^{-1}$ indicates there is no $\mathrm{C}-\mathrm{O}$ group in this monolayer. The peak at $1439 \mathrm{~cm}^{-1}$ is the $\delta_{\mathrm{a}}\left(\mathrm{CH}_{3}\right)$ mode.

\section{Agostic bonds}

We propose that the $v_{\mathrm{a}}\left(\mathrm{CH}_{2}\right)$ band at $2903 \mathrm{~cm}^{-1}$ arises from the presence of an agostic bond (metal-alkane bond) of $\mathrm{C}-\mathrm{H}$...Au. Further evidence will be provided below.

The absence of the $v_{\mathrm{s}}\left(\mathrm{CH}_{2}\right)$ and the presence of the $v_{\mathrm{a}}\left(\mathrm{CH}_{2}\right)$ strongly suggests that all the polymethylene chain must lie on the Au surface in order to have the $v_{\mathrm{s}}\left(\mathrm{CH}_{2}\right)$ dipole parallel to the $\mathrm{Au}$ surface and the $v_{\mathrm{a}}\left(\mathrm{CH}_{2}\right)$ perpendicular to the Au surface at the same time. The decrease in the peak frequency by $20 \mathrm{~cm}^{-1}$ further supports this surface $\mathrm{C}-\mathrm{H}$...Au interaction.

\section{ODT on Au covered by a sulfur monolaver}

Gold substrates were immersed in the $\mathrm{CCl}_{4}$ solution of $10 \mathrm{mM}$ of $\mathrm{Na}_{2} \mathrm{~S}$ for 4 hours and then rinsed successively with $\mathrm{CCl}_{4}$ and ethanol. These sulfur monolayers on Au were then immersed in different concentration of ODT solution for $10 \mathrm{~min}$. These Au surfaces were virtually covered by the sulfur monolayer with no bare $\mathrm{Au}$ atoms exposed at the surface ${ }^{31-33}$. This prevents the polymethylene chain from interacting with the $\mathrm{Au}$ atoms.

Obviously, only crystalline-like and liquid-like $v_{\mathrm{a}}\left(\mathrm{CH}_{2}\right)$ peaks with no such agostic bond at $2903 \mathrm{~cm}^{-1}$ at all are observed on the Au surface which was pretreated with a sulfur monolayer as shown in Figure 6. This strongly confirms that the band at $2903 \mathrm{~cm}^{-1}$ is associated with the bare $\mathrm{Au}$ atoms. Furthermore, the presence of both the $v_{\mathrm{a}}\left(\mathrm{CH}_{2}\right)$ and $v_{\mathrm{s}}\left(\mathrm{CH}_{2}\right)$ peaks concludes that the polymethylene chain axis is away from the bare $\mathrm{Au}$ atoms even at low coverage so that no agostic bond can be observed because no bare Au atoms available. 


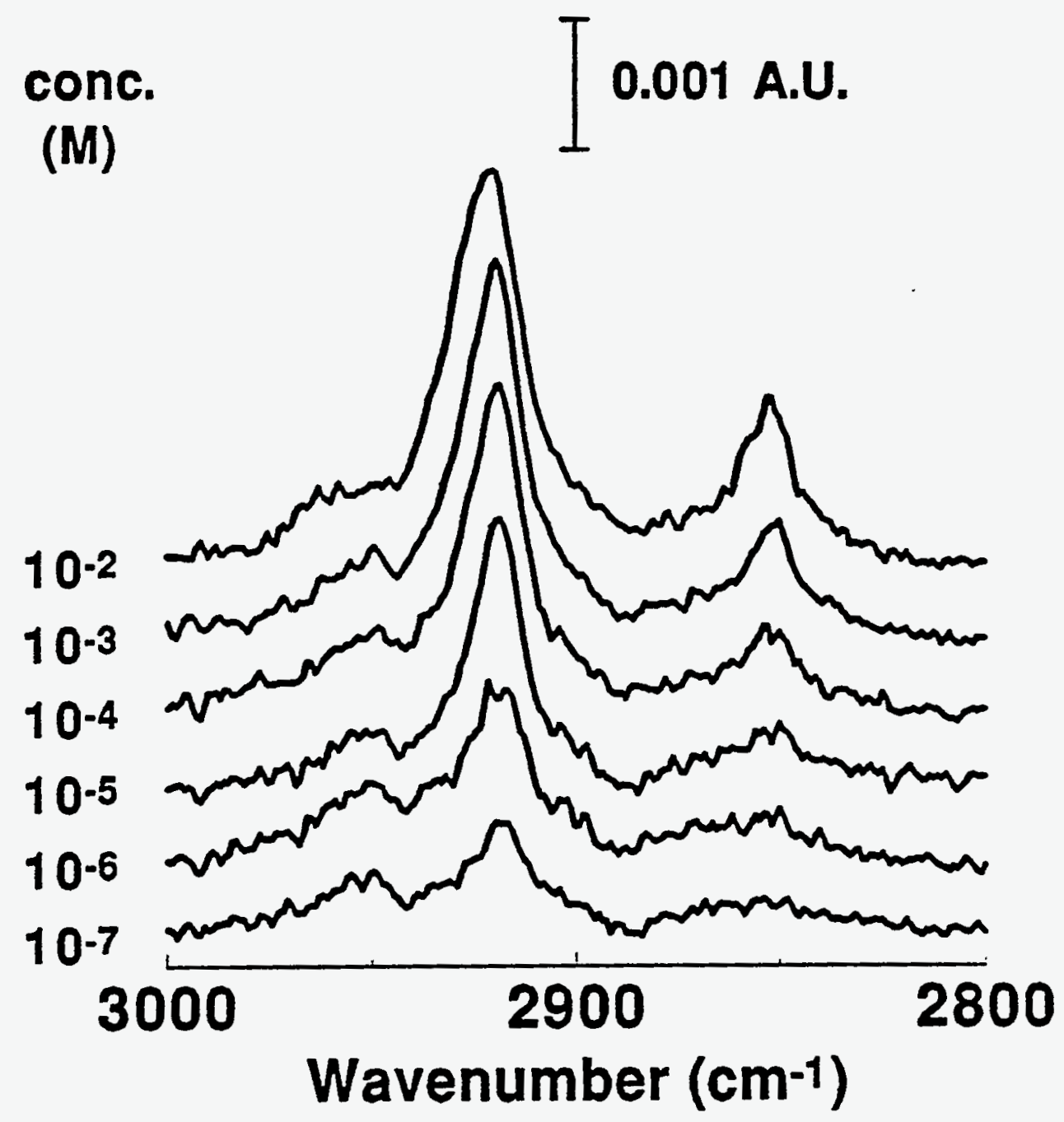

Figure 6. The IRRAS spectra in the high energy region of ODT on Au which is covered by a sulfur monolayer in different concentration of ODT for $10 \mathrm{~min}$. 
conc.

(M)

0.0005 A.U.

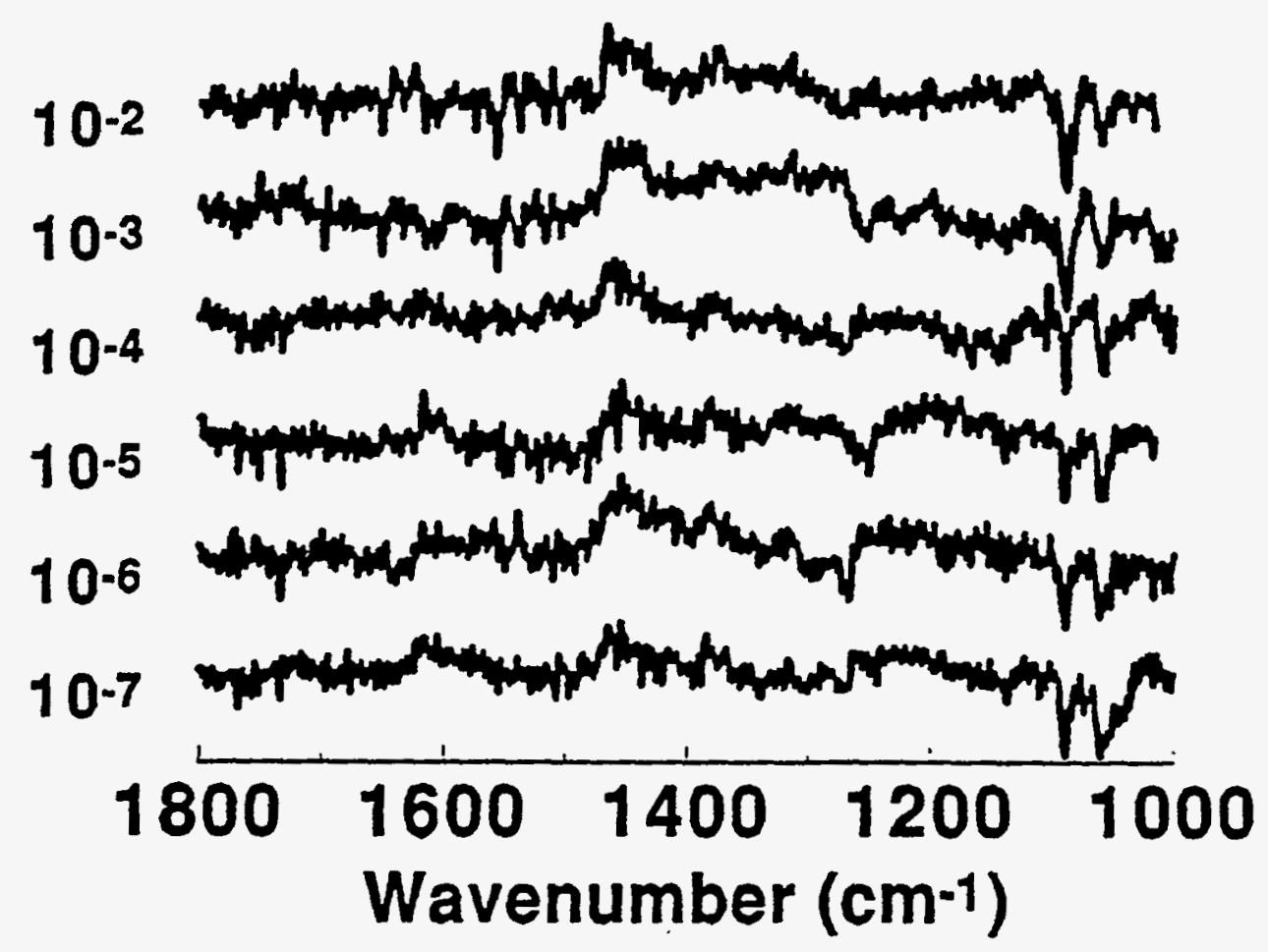

Figure 7. The IRRAS spectra in the low energy region of ODT on Au which is covered by a sulfur monolayer in different concentration of ODT for $10 \mathrm{~min}$. 
Unlike the MHA/Au and ODT/Au monolayers, ODT on Au which is covered by a monolayer of sulfur has only very little change in low energy region of IRRAS spectra with immersion time as shown in Figure 7. This is simply because of the lack of bare Au atoms which is already occupied by a sulfur monolayer. Clearly, when there is no bare Au atoms, there is no peak at $2903 \mathrm{~cm}^{-1}$ neither. This indirectly supports the formation of the agostic bond between either MHA or ODT and bare Au surface.

\section{Evidence for agostic bonds}

The $\mathrm{C}-\mathrm{H} \cdot \mathrm{\cdots} \cdot \mathrm{M}$ hydrogen bonding has been verified by using neutron diffraction ${ }^{34}$, crystal structure, NMR, and $\mathbb{R}^{35-49}$ as well. The C-H...M hydrogen bond has an energy of the order of a few $\mathrm{kcal} / \mathrm{mol}$ which is between the extremes of a chemical bond and van der Waals interactions ${ }^{50,51}$. The vibration peak of this kind of hydrogen bond has lower energy by tens to several hundred wavenumbers and is greatly broadened compared to the corresponding bands of non hydrogen-bonded group ${ }^{52-59}$. The extent of this hydrogen bond is almost proportional to the propensity for dehydrogenation:

$$
\operatorname{Ru}(001)>\operatorname{Pt}(111)>\operatorname{Pd}(111)>\mathrm{Ni}(111)>\mathrm{Cu}(111)
$$

A CH$~_{2}$ group contributes $5-6.5 \mathrm{~kJ} / \mathrm{mol}$ to the heat on $\mathrm{Pt}(111)$ and $\mathrm{Cu}(100)^{60}$. The monolayer alcohol adsorption heats increased sequentially from methanol to $\mathrm{n}$-butanol $(11.5-15 \mathrm{kcal} / \mathrm{mol})$ on $\mathrm{Pt}(111)^{61}$. The heats of adsorption increase incrementally with addition of methylene groups to the hydrocarbon chain following the formula $\Delta \mathrm{H}_{\mathrm{ad}}=8.6+1.1 \mathrm{n} \mathrm{kcal} / \mathrm{mol}$ for $\mathrm{CH}_{3}\left(\mathrm{CH}_{2}\right)_{\mathrm{n}-1} \mathrm{OH}$ on $\mathrm{Ag}(110)^{62}$. In the case of adsorption of alkylthiol on $\mathrm{Au}$ surfaces, a lower limit of $0.8 \mathrm{kcal} / \mathrm{mol}$ is estimated for the interaction between $\mathrm{CH}_{2}$ groups ${ }^{11}$. The enthalpies of the intermolecular agostic bond between heptane and $\mathrm{Cr}, \mathrm{Mo}, \mathrm{W}$ are calculated to be $10,9,13 \mathrm{kcal} / \mathrm{mol}$, respectively ${ }^{63}$.

The agostic bonds between alcohols and $\mathrm{Ag}$ were found by using surface-enhanced Raman scattering spectroscopy ${ }^{64,65}$ by Pemberton and et al. The $v_{s}\left(\mathrm{CH}_{2}\right)$ agostic bond bands were shifted from $2850 \mathrm{~cm}^{-1}$ down to 2830 $\mathrm{cm}^{-1}$. SERS data suggest that all four alchols studied are oriented with the $\alpha-$ carbon confined near the surface at all potentials studied. they proposed that 
the driving force for this is the formation of $\mathrm{CH}_{2} \cdots \mathrm{Ag}$ agostic bonds. In fact, those bands at $2900 \mathrm{~cm}^{-1}$ that they founded are the agostic bond of $v_{\mathrm{a}}\left(\mathrm{CH}_{2}\right)$ which is shifted down from $2928 \mathrm{~cm}^{-1}$ as well although they did not point this out ${ }^{65}$.

\section{The evolution of the structure of MHA/Au and ODT/Au}

The polymethylene chain

The presence of the $v_{\mathrm{s}}\left(\mathrm{CH}_{2}\right)$ is at the expanse of the amount of $v_{\mathrm{a}}\left(\mathrm{CH}_{2}\right)$ as shown in Figures 1 and 3. This is because the polymethylene chain must turn the chain axis in order to stand up to accommodate more coverage so that the $v_{\mathrm{s}}\left(\mathrm{CH}_{2}\right)$ dipole will have more and more vertical component to the surface and the $v_{a}\left(\mathrm{CH}_{2}\right)$ dipole will have less and less vertical component to the surface simultaneously. This can be confirmed by the substitution of the surface agostic $v_{a}\left(\mathrm{CH}_{2}\right)$ at $2903 \mathrm{~cm}^{-1}$ by the liquid-like $v_{\mathrm{a}}\left(\mathrm{CH}_{2}\right)$ at $2928 \mathrm{~cm}^{-1}$ when the coverage increases.

At immersion time of $\sim 720 \mathrm{~min}$, the agostic $v_{\mathrm{a}}\left(\mathrm{CH}_{2}\right)$ peak of MHA $/ \mathrm{Au}$ disappears and the peak frequency of the $v_{\mathrm{a}}\left(\mathrm{CH}_{2}\right)$ shifts from $2925 \mathrm{~cm}^{-1}$ to $2920 \mathrm{~cm}^{-1}$ as shown in Figure 1. This substantial decrease in peak frequency indicates the polymethylene chain changes from a liquid-like structure to a crystalline-like environment only when the agostic species totally disappears ${ }^{66}$. This phenomenon implies that the polymethylene chain cannot form the most densely-pack structure as long as there are any agostic molecules because agostic molecules occupy the surface and prevent the polymethylene chain from gathering together to maximize the $\mathrm{CH}_{2}$ interactions unless the coverage is great enough so that the total $\mathrm{CH}_{2}$ interactions is greater than the agostic bond. The same trend is observed for ODT on $\mathrm{Au}$ as shown in Figure 3.

For both monolayers with agostic bonds at very low coverage, peaks found at $1396 \mathrm{~cm}^{-1}, 1436 \mathrm{~cm}^{-1}$ are $\delta\left(\alpha-\mathrm{CH}_{2}\right)$ and $\gamma(\mathrm{C}-\mathrm{O})$, respectively, as shown in Figures 2 and 8 . This suggests that the polymethylene chain not only lies on the Au surface but also have the $\alpha-\mathrm{CH}_{2}$ twisted so that the $\delta\left(\alpha-\mathrm{CH}_{2}\right)$ has more perpendicular component to the surface with the $\mathrm{C}-\mathrm{O}$ bond more perpendicular to the surface at the same time. 
The decrease in the intensity of the $\delta\left(\alpha-\mathrm{CH}_{2}\right)$ and $r(\mathrm{C}-\mathrm{O})$ peaks with increasing the coverage suggests that the polymethylene chain must turn around to decrease the peak intensities. This is consistent with the conclusion form the $v\left(\mathrm{CH}_{2}\right)$ analysis.

The increases in the peak intensities of $\omega\left(\mathrm{CH}_{2}\right)$ peaks at $1344 \mathrm{~cm}^{-1}$ and $1380 \mathrm{~cm}^{-1}$ and $\delta\left(\mathrm{CH}_{2}\right)$ at $1460 \mathrm{~cm}^{-1}$ with dipole in the polymethylene chain axis suggests that the polymethylene chain stands up with increasing the coverage.

The carbonyl stretching mode

Only MHA/Au has carbonyl at $1717 \mathrm{~cm}^{-1}$ does not appear at very low coverage as a consequence of the twist of the $\alpha-\mathrm{C}$ and the turning of $\mathrm{CO}_{2} \mathrm{H}$ with $\checkmark(\mathrm{C}-\mathrm{O})$ dipole almost perpendicular to the $\mathrm{Au}$ surface.

The carboxylic acid group is partially deprotonated in ethanol solution since in addition to the carbonyl band at $1717 \mathrm{~cm}^{-1}$, two carboxylate bands at $\sim 1580 \mathrm{~cm}^{-1}$ and $\sim 1450 \mathrm{~cm}^{-1}$ exist at high coverage as shown in Figure 2. 


\section{CONCLUSIONS}

At very low coverage, all the polymethylene groups lie on the $\mathrm{Au}$ surface and form the $\mathrm{C}-\mathrm{H}$...Au agostic bonds which have the $\mathrm{v}_{\mathrm{a}}\left(\mathrm{CH}_{2}\right)$ band at $2903 \mathrm{~cm}^{-1}$ with the $v_{\mathrm{s}}\left(\mathrm{CH}_{2}\right)$ dipole oriented parallel to the $\mathrm{Au}$ surface and the $v_{a}\left(\mathrm{CH}_{2}\right)$ dipole oriented perpendicular to the Au surface at the same time and the $v(\mathrm{C}-\mathrm{O})$, and the $\mathrm{r}(\mathrm{C}=\mathrm{O})$ dipoles of MHA/Pt oriented almost perpendicular, and parallel to the Au surface, respectively. The $\alpha-\mathrm{CH}_{2}$ is twisted so that the $\delta\left(\alpha-\mathrm{CH}_{2}\right)$ has more perpendicular component to the surface with the $\mathrm{C}-\mathrm{O}$ bond pointing more perpendicular to the surface at the same time.

With increases in coverage, submonolayers must have the polymethylene chain turn the chain axis more and more in order to stand up higher and higher away from the surface in order to allow more and more molecules to be adsorbed on the surface. Eventually, all the agostic bonds will disappear. 


\section{REFERENCES}

(1) Bain, C. D.; Whitesides; G. M. Angew. Chem., Int. Ed. Engl. 1989, 28, 506 , and references therein.

(2) Whitesides, G. M.; Laibinis, P. E. Langmuir 1990, 6, 87, and references therein.

(3) Ulman, A. An Introduction to Ultra-Thin Organic Films From Langmuir-Blodgett to Self-Assembly; Academic Press: San Diego, 1991.

(4) Porter, M. D.; Bright, T. B.; Allara, D. L.; Chidsey, C. E. D. J. Am. Chem. Soc. 1987, 109, 3559.

(5) Troughton, E. B.; Bain, C. D.; Whitesides, G. M.; Nuzzo, R. G.; Allara, D. L.; Porter, M. D. Langmuir 1988, 4, 365.

(6) Walczak, M. M.; Chung, C.; Stole, S. M.; Widrig, C. A.; Porter, M. D. J. Am. Chem. Soc. 1991, 113, 2370.

(7) Widrig, C. A.; Alves, C. A.; Porter, M. D. J. Am. Chem. Soc. 1991, 113, 2805.

(8) Alves, C. A.; Smith, E. L.; Porter, M. D. J. Am. Chem. Soc. 1992, 114, 1222.

(9) Widrig, C. A.; Chung, C.; Porter, M. D. J. Electroanal. Chem. 1991, $310,335$.

(10) Dubois, L. H.; Zegarski, B. R.; Nuzzo, R. G. J. Chem. Phys. 1993, 98, 678.

(11) Nuzzo, R. G.; Dubois, L. H.; Allara, D. L. J. Am. Chem. Soc. 1990, $112,558$.

(12) Strong, L.; Whitesides, G. M. Langmuir 1988, 4, 546.

(13) Bain, C. D.; Whitesides, G. M. J. Am. Chem. Soc. 1988, 110, 5897.

(14) Bryant, M. A.; Pemberton, J. E. J. Am. Chem. Soc. 1991, 113, 8284.

(15) Buck, M.; Eisert, F.; Fischer, J.; Grunze, M.; Träger, F. Appl. Phys. 1991, $A 53,552$.

(16) Hähner, G.; Wöll, Ch.; Buck, M.; Grunze, M. Langmuir 1993, 9, 1955.

(17) Hähner, G.; Kinzler, M.; Thümmler, C.; Wöll, Ch.; Grunze, M. J. Va. Sci. Technol. 1992, A10, 2758.

(18) Shimazu, K.; Yagi, I.; Uosaki, K. Langmuir 1992, 8, 1385. 
(19) Cardini, G.; Bareman, J. P.; Klein, M. L. Chem. Phys. Lett. 1988, 145, 493.

(20) Bareman, J. P.; Cardini, G.; Klein, M. L. Phys. Rev. Lett. 1988, 60, 2152.

(21) Hautman, J.; Klein, M. L. J. Chem. Phys. 1989, 91, 4994.

(22) Ulman, A.; Eilers, J. E.; Tillman, N. Langmuir, 1989, 5, 1147.

(23) Chidsey, C. E. D.; Liu, G.-Y.; Rowntree, P.; Scoles, G. J. Chem. Phys. 1989, 91, 4421

(24) Bain, C. D.; Troughton, E. B.; Tao, Y-T.; Evall, J.; Wihitesides, G. M.; Nuzzo, R. G. J. Am. Chem. Soc. 1989, 111, 321.

(25) Buck, M.; Eisert, F.; Fischer, J. Grunze, M.; Träger, F. J. Vac. Sci. Technol. 1992, A10, 926.

(26) Thomas, R. C.; Sun, L.; Crooks, R. M.; Ricco, A. J. Langmuir 1991, 7, 620.

(27) Nuzzo, R. G.; Dubois, L. H.; Allara, D. L. J. Am. Chem. Soc. 1990, $112,558$.

(28) Azzam, G. A.; Bashara, N. M. Ellipsometry and Polarized Light; North-Holland Publishing: Amsterdam, 1977.

(29) Ulman, A. J. Mater, Educ. 1989, 11, 205.

(30) Stole, S. M.; Porter, M. D. Appl. Spectrosc. 1990, 44, 1418.

(31) Gao, X.; Zhang, Y.; Weaver, M. J. Langmuir 1992, 8, 668.

(32) Briceno, A.; Chander, S. J. Appl. Electrochemistry 1990, 20, 506.

(33) Briceno, A.; Chander, S. J. Appl. Electrochemistry 1990, 20, 512.

(34) Brown, R. K.; Williams, J. M.; Schultz, A. J.; Stucky, G. D.; Illel, S. D.; Harlow, R. L. J. Am. Chem. Soc. 1980, 102, 981.

(35) Roe, D. M.; Bailey, P. M.; Moseley, K.; Maitlis, P. M. J. Chem. Soc. Chem. Comm. 1972, 1273.

(36) Cotton, F. A.; Jeremic, M.; Shaver, A. Inorg. Chim. Acta 1972, 6, 543.

(37) Cotton, F. A.; LaCour, T.; Stanislowski, A. G.; J. Am. Chem. Soc. 1974, 96, 754.

(38) Cotton, F. A.; Day, V. W.; J. Chem. Soc. Chem. Comm. 1974, 415.

(39) Ittel, S. D.; Van-Catledge, F. A.; Jesson, J. P. J. Am. Chem. Soc. 1979, 101,6905 .

(40) Tachikawa, M.; Muetterties, E. L. J. Am. Chem. Soc. 1980, 102, 4542. 
(41) Dawkins, G. M.; Green, M.; Orpen, A. G.; Stone, F. G. A.; J. Chem. Soc. Chem. Comm. 1982, 412.

(42) Dawoodi, Z.; Green, M. L. H.; Mtetwa, V. S. B.; Prout, J. Chem. Soc. Chem. Comm. 1982, 802.

(43) Dawoodi, Z.; Green, M. L. H.; Mtetwa, V. S. B.; Prout, J. Chem. Soc. Chem. Comm. 1982, 1410.

(44) Schultz, A. J.; Williams, J. M.; Schrock, G. Rupprecht, G.; Fellmann, J. D. J. Am. Chem. Soc. 1979, 101, 1593.

(45) Schultz, A. J.; Brown, R. K.; Williams, J. M.; Schrock, J. Am. Chem. Soc. 1981, 103, 169.

(46) Schrock, R. R.; Fellmann, J. D. J. Am. Chem. Soc. 1978, 100, 3519.

(47) Wengovius, J. H.; Schrock, R. R.; Churchill, M. R.; Wasserman, H. J.; J. Am. Chem. Soc. 1982, 104, 1739.

(48) Itoh, K.; Oshima, N.; Jameson, G. B.; Lewis, H. C.; Tbers, J. A. J. Am. Chem. Soc. 1981, 103, 3014.

(49) Turner, H. W.; Schrock, R. R.; J. Am. Chem. Soc. 1982, 104, 2331.

(50) Pimental, G. C.; McClellan, A. L. The Hydorgen Bond; Freeman: San Fransisco, 1960.

(51) Schuster, P.; Zundel, G.; Snadorfy, C. The Hydrogen Bond, Vols I, II, and III, 1976.

(52) Demuth, J. E.; Ibach, H.; Lehwald, S. Phys. Rev. Letters 1978, 40, 1044.

(53) Chesters, M. A.; Parker, S. F.; Raval, R. J. Electron Spectrosc. Related Phenomena 1986, 39, 155.

(54) Raval, R.; Pemble, M. E.; Chesters, M. A. Surface Sci. 1989, 210, 187.

(55) Lehwald, S.; Ibach, H. Surface Sci. 1979, 89, 425.

(56) Hoffmann, F. M.; Felter, T. E.; Thiel, P. A.; Weinberg, W. H. Surface Sci. 1983, 130, 173.

(57) Waddill, G. D.; Kesmodel, L. L. Chem. Phys. Letters 1986, 128, 208.

(58) Felter, T. E.; Hoffermann, F. M.; Thiel, P. A.; Weinberger, W. H. Surface Sci. 1983, 130, 163.

(59) Raval, R.; Chesters, M. A. Surface Sci. 1989, 219, L505.

(60) Shustorovich, E.; Baetzold, R. C.; Muetterties, J. Phys. Chem. 1983, $87,1100$. 
(61) Sexton, B. A.; Rendulic, K. D.; Hughes, A. E. Surface Sci. 1982, 121, 181.

(62) Zhang, R.; Gellman, A. J. J. Phys. Chem. 1991, 95, 7433.

(63) Mores, J. M.; Parker, G. H.; Burkey, T. J. Organometallies 1989, 8, 2471.

(64) Sobocinski, R. L.; Pemberton, J. E. Langmuir 1992, 8, 2049.

(65) Joa, S. L.; Pemberton, J. E. Langmuir 1992, 8, 2301.

(66) Snyder, R. G.; Strauss, H. L.; Elliger, C. A. J. Phys. Chem. 1982, 86, 5145. 


\section{INTRODUCTION}

The most attractive beauty of these self-assembly monolayers is that these thiolate monolayers are not only very densely-packed, but also very stable $^{1-3}$. Furthermore, it is not only possible to change the tail group but also very easy to modify the end group for application purpose such as non-linear and active optical devices, chemical, biochemical and physical sensors, protective layers, patternable materials both for resists and for mass information storage, surface modification (e.g. wetting and electrochemical electrode properties) ${ }^{4}$. All these ideal properties of self-assembled monolayers have attracted worldwide attention. As a consequence, the study of the properties and construction of the self-assembled thiol monolayers on gold has grown enormously in recent years ${ }^{5-7}$. Although there are many different methods of preparing surface monolayers available including LangmuirBlodgett technique ${ }^{8-10}$. The self-assembled monoalyers on Au have been well characterized.

Porter and co-workers used infrared reflection absorption spectroscopy (IRRAS), ellipsometry and contact angle measurements to demonstrate that the tilt angle is $30^{\circ}$ with twist angle of $50^{\circ}$ and there is an odd-even chain-lengthdependent orientation of the methyl group which provides the best evidence of a conserved $\mathrm{Au}-\mathrm{S}-\mathrm{C}$ bond angle $\mathrm{e}^{11-13}$. Monolayers have a well ordered pattern of $\sqrt{3} \times \sqrt{3} \mathrm{R} 30^{\circ}$ with $5.0 \AA$ separation to the nearest neighbor from STM imagine $^{14,15}$. The packing density of these is $8.0 \times 10^{-10} \mathrm{~mol} / \mathrm{cm}^{2}$ on $\mathrm{Au} / \mathrm{mica}$ annealed at $300^{\circ} \mathrm{C}$ assuming roughness factor of 1.2. This suggests these monolayers have $\sqrt{3} \times \sqrt{3} \mathrm{R} 30^{\circ}$ structure with $30^{\circ}$ tilt angle from the surface normal ${ }^{16}$. The loss of the mercapto hydrogen and formation of a gold alkanethiolate was concluded ${ }^{16}$.

Whitesides and co-worker have shown that the distance between the nearest neighbor is $4.97 \pm 0.05 \AA$ by transmission electron diffraction spectroscopy ${ }^{17}$.

Nuzzo et al. have used low-energy electron diffraction (LEED) and concluded that these monolayers have a $\sqrt{3} \times \sqrt{3} \mathrm{R} 30^{\circ}$ with $4.99 \AA$ structure $^{18}$. These molecular architecture is adopted independent of the chain 
length or chain-terminating group and the tilt angle is $\sim 30^{\circ}$ with twist angle of $52^{\circ}$ which implies that the Au surface-sulfur-carbon bond angle is near $120^{\circ} .{ }^{19}$

The 16-mercaptohexadecanoic acid monolayer on $\mathrm{Au}$ has been characterized by Nuzzo et al. ${ }^{19}$ They concluded the structure of MHA/Au monolayer is an almost perfect zigzag (all trans) conformation. From the IRRAS $\mathrm{CH}_{2}$ stretching modes, they calculated the tilt angle of $32^{\circ}$ and twist angle of $55^{\circ}$ with thickness of $22 \AA$. The axis of $\mathrm{C}=\mathrm{O}$ is tilted form the surface normal by $66^{\circ}$.

The usage of the monolayers is determined by their surface properties which are a combined result of the coverage, the orientation, and the composition of the end groups. A good understanding of the relationship between the surface properties of the monolayers and the coverage, orientation of a given end group of monolayers is very essential to fabricate monolayers with desired properties.

In this study, a carboxyl acid-terminated thiolate monolayer on $\mathrm{Au}$ $\left(\mathrm{HO}_{2} \mathrm{C}\left(\mathrm{CH}_{2}\right)_{15} \mathrm{~S} / \mathrm{Au}\right)$ was studied using IRRAS as a function of immersion time to examine the change in the relative amount of the non-hydrogen-bonded $\mathrm{C}=\mathrm{O}$, the hydrogen-bonded dimeric $\mathrm{C}=\mathrm{O}$, and hydrogen-bonded polymeric $\mathrm{C}=\mathrm{O}$ as a function of coverage. The dramatical changes in wettability and ellipsometric thickness of the monolayer using contact angles and ellipsometric thickness measurements as a function of immersion time are reported. 


\section{EXPERIMENTAL SECTION}

\section{Substrate preparation}

Electrodes were prepared by the resistive evaporation of $15-20 \mathrm{~nm}$ of chromium, followed by 200-300 nm of gold, onto 3 inch by 1 inch glass substrates. The temperature during the evaporation, measured at the plate supporting the substrates, increased to $\sim 50^{\circ} \mathrm{C}$ as a result of radiative heating by the evaporation source. The evaporation rates were 0.2 and $2.0 \mathrm{~nm} / \mathrm{s}$ for chromium and gold, respectively. The pressure in a cryopumped E360A Edwards Coating System during evaporation was $<9 \times 10^{-5} \mathrm{~Pa}$. After $\sim 45 \mathrm{~min}$ cooling time, the evaporator was back-filled with purified $\mathrm{N}_{2}$. The substrates were then removed, analyzed by optical ellipsometry for the determination of the optical functions of the uncoated substrates, and immersed in the thiolcontaining solution.

The surfaces of the uncoated gold electrodes have been characterized previously at both a macroscopic and microscopic level using scanning tunneling microscopy ${ }^{20}$ and electrochemical measurements of surface texture and surface area ${ }^{16}$. Macroscopically, the STM images show that the electrode surface is composed of atomically-terraced crystallites $\sim 25 \mathrm{~nm}$ in diameter. The electrodes have a roughness factor of $\sim 1.3$, which is given as the surface area determined from the oxidative desorption of iodine ${ }^{16}$ divided by the geometric electrode area.

\section{Monolaver preparation}

The carboxylic acid-,and methyl-terminated alkanethiolate monolayers were prepared by their spontaneous adsorption onto the evaporated gold from $0.100 \mathrm{mM}$ solutions in absolute ethanol. For hydrogen-bonded $\mathrm{C}=\mathrm{O}$ monolayers, the substrates were placed in solution for $4.00 \mathrm{~min}$, emersed, rinsed successively with absolute ethanol and hexane, and dried under a stream of prepurified $\mathrm{N}_{2}$. To form free $\mathrm{C}=\mathrm{O}$, longer time is needed. 


\section{Emersion measurements}

The Emerson experiments were performed by the slow withdrawal of the monolayer-coated substrates from aqueous solutions of varied $\mathrm{pH}$. Typically, samples were left in solution for $\sim 1 \mathrm{~min}$, with immersions of up to $\sim 1$ h yielding essentially equivalent results. Upon removal from solution, the samples were placed on a spin-coater and spun briefly at $2000 \mathrm{rpm}$ to remove excess solution. The samples were then placed in the $\mathrm{N}_{2}$-purged sample chamber of the IR spectrometer for characterization. Spectra were usually acquired within a few minutes of loading in the chamber, with the rotational vibrational bands of vapor phase water subtracted by using a reference vapor spectrum. All solutions were prepared at an ionic strength of $0.100 \mathrm{M}$ by using $\mathrm{KCl}$. Comparable results were obtained in both buffered and unbuffered solution.

\section{Ellipsometric measurements of film thickness}

The thicknesses of the monolayers were determined by optical ellipsometry at $632.8 \mathrm{~nm}$ in two steps with a computer-interfaced Gaertner Model L-116B ellipsometer. The angle of incidence was $70^{\circ}$ from the surface normal. Upon removal from the evaporation chamber, the analyzer and polarizer angles for a reflected light beam from each uncoated substrate were measured on at least three different spots. The average complex refractive index for each substrate was then calculated with a two phase parallel layer model from classical electromagnetic theory ${ }^{21}$. After monolayer formation, each sample was again analyzed and the film thickness calculated from a threephase parallel layer model, using the average complex refractive index of the individual sample and a real refractive index of 1.45 for the film. A value of 1.45 , which is representative of the adsorbate precursors ${ }^{22,23}$, facilitates comparison with thickness data that has been reported for a variety of monolayers. The influence of several refinements on this treatment of ellipsometric data have been recently discussed ${ }^{11,24}$.

\section{Contact angle measurements}

Advancing contact angles $\left(\theta_{\mathrm{a}}\right)$ were measured in air with $\mathrm{pH}=1.0 \mathrm{HCl}$ aqueous solution as probe liquids by using a Rame-Hart Model 100-00 115 
goniometer. For these measurements, a $2.00 \mathrm{~mL}$ droplet was formed on the substrate with the needle of the syringe in the droplet. The value of $q_{\mathrm{a}}$ was determined as the volume of the droplet was slowly increased.

\section{Infrared spectroscopy}

Infrared spectra were acquired with either a Nicolet 740 or a 750 FT-IR spectrometer. Spectra of the adsorbate precursors were obtained by their dispersion in $\mathrm{KBr}$. Spectra of the monolayers were obtained in a single reflection mode by using p-polarized light incident at $82^{\circ}$ from the surface normal and a liquid nitrogen cooled $\mathrm{HgCdTe}$ detector. A home-built sample holder was used to position reproducibly the substrates in the sample chamber of the spectrometer ${ }^{25}$. The spectrometer was purged with boil-off from liquid $\mathrm{N}_{2}$. Spectra were obtained by referencing 1024 sample scans to 1024 background scans at $2 \mathrm{~cm}^{-1}$ resolution (zero filled) with Happ-Genzel apodization. All spectra are reported as $-\log \left(R / R_{0}\right)$, where $R$ is the reflectance of the coated substrate and $R_{0}$ is that of a reference $A u$ substrate. The reference substrates were prepared by immersion of an uncoated gold substrate into $\sim 1 \mathrm{mM}$ ethanolic solution of octadecanethiol- $\mathrm{d}_{37}$. These films provide a substrate free from detectable IR bands in the regions relevant to these studies as determined when referenced to an uncoated $\mathrm{Au}$ substrate that was cleaned in a X 1:3 $\mathrm{H}_{2} \mathrm{O}_{2}(30 \%): \mathrm{H}_{2} \mathrm{SO}_{4}$ (con.) solution. Further details of the preparation of the reference substrates are given elsewhere ${ }^{13}$. Caution: The $\mathrm{H}_{2} \mathrm{O}_{2} / \mathrm{H}_{2} \mathrm{SO}_{4}$ solution reacts violently with organic compounds and should be handled with extreme care.

The orientation analysis, which compares the observed spectrum to that calculated for an isotropic array of oscillators of comparable packing density, was accomplished by using the frequency-dependent complex optical constants of the adsorbate precursor or a suitable substitute. The optical constants for 16-mercaptohexadecanoic acid were determined from a transmission spectrum after its dispersion as a crystalline material in $\mathrm{KBr}$. From the absorbance values, a Kramers-Kronig transformation ${ }^{26}$ yielded the frequency-dependent complex optical constant. The optical constants of octadecanethiol from a comparable data workup were used for tetradecanethiol, which is a liquid at room temperature. 


\section{Chemicals and reagents}

Mercaptohexadecanoic acid (MHA) $\left(\mathrm{HO}_{2} \mathrm{C}\left(\mathrm{CH}_{2}\right)_{15} \mathrm{SH}\right)$ was synthersized in this groupand characterized with $\mathrm{IR}$ and NMR. The n-pentylundecanoic acid disulfide (PUAD) $\left(\mathrm{CH}_{3}\left(\mathrm{CH}_{2}\right)_{4}\right) \mathrm{SS}\left(\left(\mathrm{CH}_{2}\right)_{10} \mathrm{CO}_{2} \mathrm{H}\right)$ was a gift from Dr. Fred Sun (Oregon Graduate Institute of Science \& Technology). The rest of chemicals was obtained from Aldrich. The alkanethiols were purified prior to use by passage through an activity one neutral alumina column. All other reagents were used as received. Aqueous solutions were freshly prepared from water purified by passage through a Milli-Q purification system (Millipore, Bedford, MA). 


\section{RESULTS AND DISCUSSION}

\section{General observations of monolayer formation}

Ellipsometric thickness as a function of immersion time

The thickness of the MHA monolayer on gold (MHA/Au) was measured as a function of immersion time using optical ellipsometry. The results are shown in Figure 1. In $0.1 \mathrm{mM}$ MHA solution, the thickness increases rapidly at short immersion times, reaching a value of $\sim 9 \AA$ within $0.1 \mathrm{~min}$. The rate of the process slows shortly thereafter with the thickness doubling within $\sim 30 \mathrm{~min}$ and approaching a limiting value of $22.0 \AA$.

This formation process is not a simple one step Langmuir adsorption surface process because it cannot be fitted by using only one single Langmuir isotherm. Actually, this result is consistent with the two step formation process proposed by Whitesides et al. ${ }^{27}$ and confirmed by Grunze et al. ${ }^{28-29}$, Shimazu et al. $^{30}$, and Thomas et al. ${ }^{31}$ The first step can be viewed as a Langmuir adsorption process, and the second step is believed to be a result of the combination of replacement of the incorporated solvent and adsorbed impurities and lateral diffusion on the surface to reduce defects and enhance packing $^{27}$. This second process has a much slower rate than that of the first process.

\section{Wetting as a function of immersion time}

The wetting property of the surface of MHA/Au as a function of immersion time was characterized with contact angle measurements. The contact angle of water $\left(\theta_{\mathrm{a}}\right)$ and the value of $\cos \theta_{\mathrm{a}}$ as a function of immersion time as shown in Figure 2. The contact angle decreases dramatically from 57.0 to 18.0 in less than $10 \mathrm{~min}$ and decreases only slightly thereafter.

A plot of the value of $\cos \theta_{a}$ vs. ellipsometric thickness is shown in Figure 3. Clearly, when the thickness is less than $15 \AA$, the value of $\cos \theta_{a}$ increases dramatically and almost linearly with increasing the ellipsometric thickness. When the thickness is greater than $15 \AA, \cos \theta_{a}$ levels off and approaches a limiting value of $0.96\left(\theta_{\mathrm{a}}=16.0\right)$.

These observations clearly demonstrate that the surface properties of a monolayer are determined by its structure even though there is no change in the 


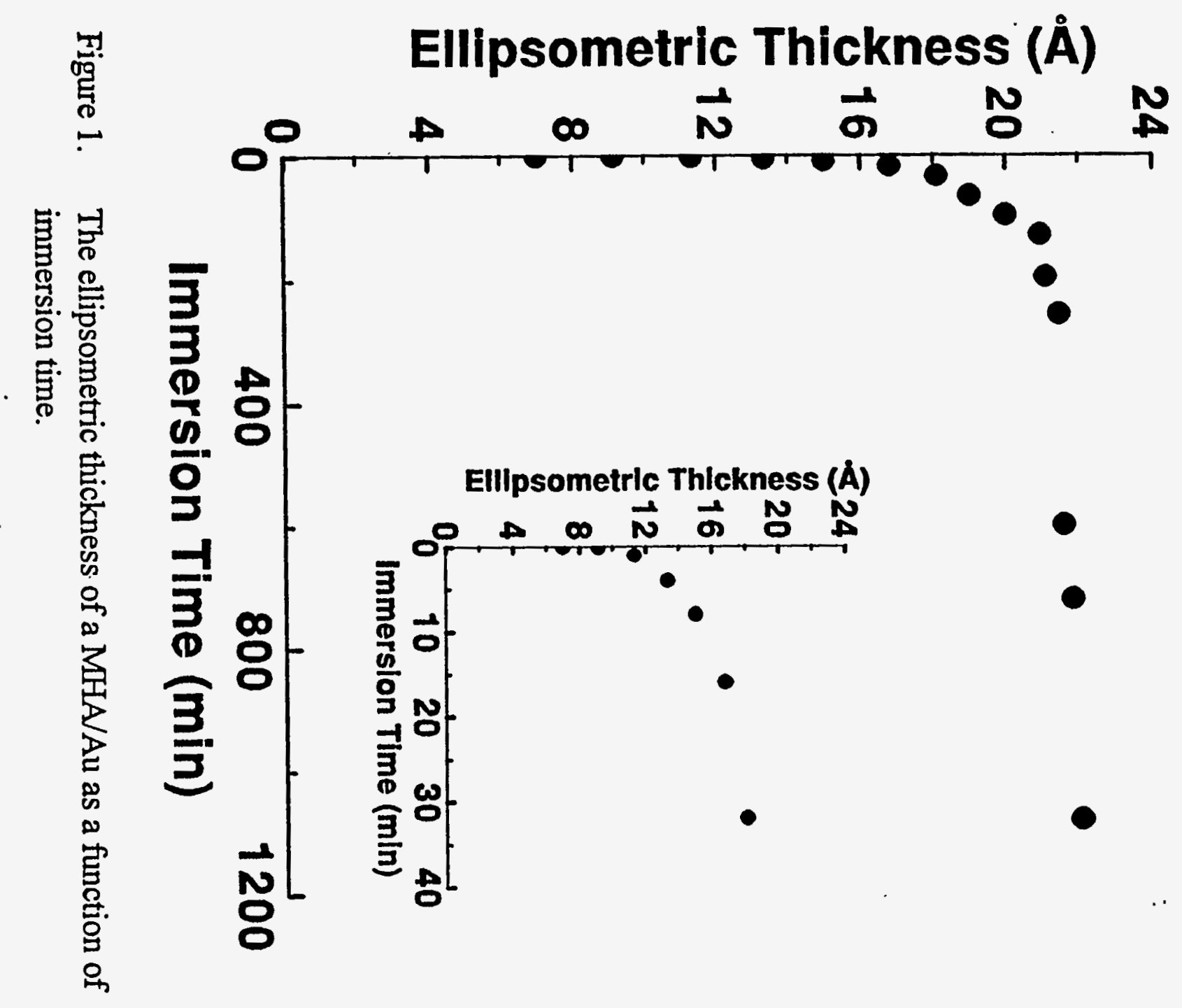




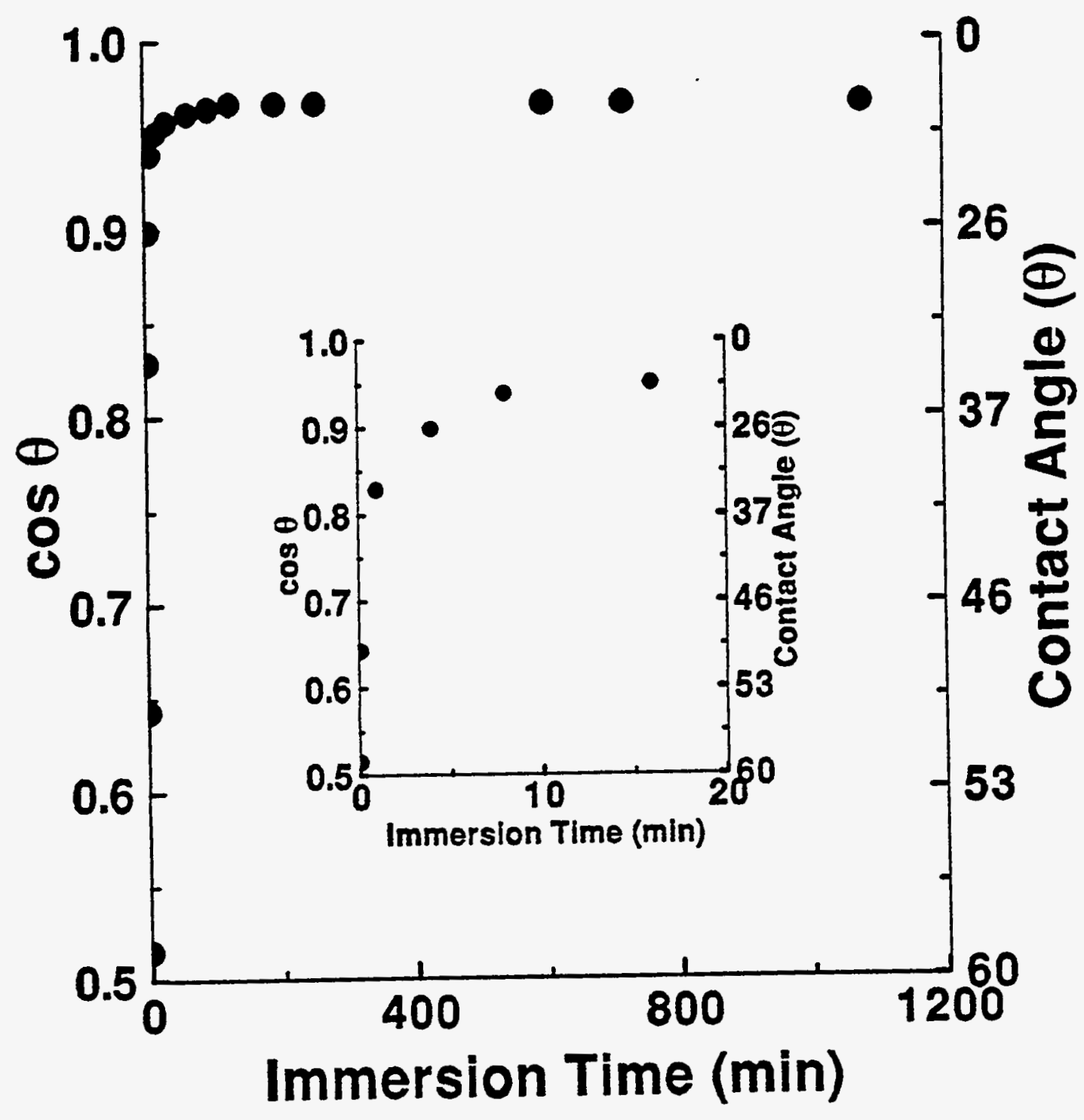

Figure 2. $\cos \theta_{\mathrm{a}}$ of water at a MHA/Au surface at $\mathrm{pH} 1.0$ as a function of immersion time. 


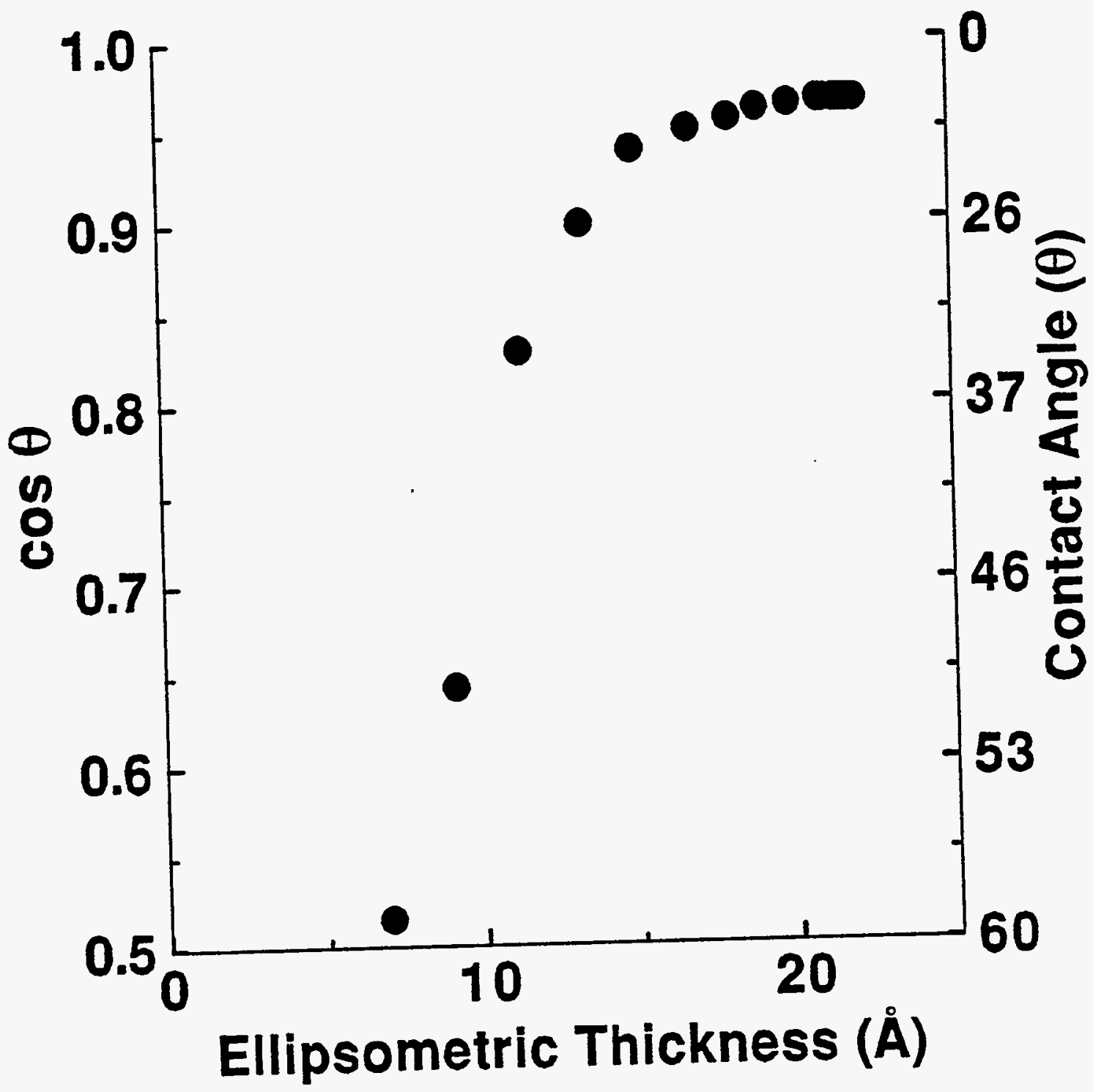

Figure 3. $\cos \theta_{\mathrm{a}}$ of water at a MHA/Au surface at $\mathrm{pH} 1.0$ as a function of the ellipsometric thickness. 
composition of this monolayer.

One of the most important issues in this field of study at present time is to be able to control the architecture of a monolayer (i.e. the structure of a monolayer) in order to fabricate materials for research and/or application purposes. To achieve this goal, it is essential to have a detailed understanding how the monolayer structure correlate to surface properties, as discussed above. Gaining an understanding the relatioship between the monolayer structure and the surface properties is the focus of this study.

The value of $\cos \theta_{\mathrm{a}}$ at $0 \AA$ thickness, from the extrapolation in Figure 19 , is $\sim 0.25$. Thus, the contact angle at $0 \AA$ thickness is $\sim 75^{\circ}$ which is about the same value of water on a polymethylene alcohol surface ${ }^{32}$. It is therefore reasonable to ascribe the nearly linear increase in the values of $\cos \theta_{\mathrm{a}}$ from 0.25 up to 0.95 is as a result of the increases in the ellipsometric thickness of the film going from $0 \AA$ to $15 \AA$. This behavior indicates that when the coverage is low, the surface appears polymethylene. At low coverage, the monolayer structure would therefore consist of molecules laying down against one another with a very large tilt angle. This proposed structure will be supported shortly by the tilt angle analysis. With increasing coverage, the surface properties are dominated by the carboxylic acid groups on the surface.

\section{IRRAS spectra as a function of immersion time}

Figure 4 is a series of IRRAS spectra of MHA/Au as a function of immersion time. There are two main peaks and a shoulder around $2905 \mathrm{~cm}^{-1}$. The peak at $2920 \mathrm{~cm}^{-1}$ is assigned to the asymmetric $\left(\mathrm{CH}_{2}\right)$ mode, and the other peak at $2850 \mathrm{~cm}^{-1}$ is the symmetric $v\left(\mathrm{CH}_{2}\right)$ mode $\mathrm{C}^{31}$. The shoulder at $2903 \mathrm{~cm}^{-1}$ is an IR peak which has not yet been discovered and assigned in the literature (see below).

Figures 4-6 show a marked change of $r\left(\mathrm{CH}_{2}\right)$ and $\mathrm{r}(\mathrm{C}=0)$ as a function of immersion time. In the carbonyl stretching region $(1600-1750 \mathrm{~cm})$, the spectrum transfers from a single band at $1717 \mathrm{~cm}^{-1}$ to a doublet. The low energy band at $1717 \mathrm{~cm}^{-1}$, assigned to a hydrogen-bonded dimeric $\mathrm{C}=\mathrm{O}$ mode of a carboxylic acid (see below), decreases with increasing immersion time. The band at $1745 \mathrm{~cm}^{-1}$, assigned to a non-hydrogen-bonded $\mathrm{C}=\mathrm{O}$ mode of a carboxylic acid, i.e. a free acid, increases with immersion time. 


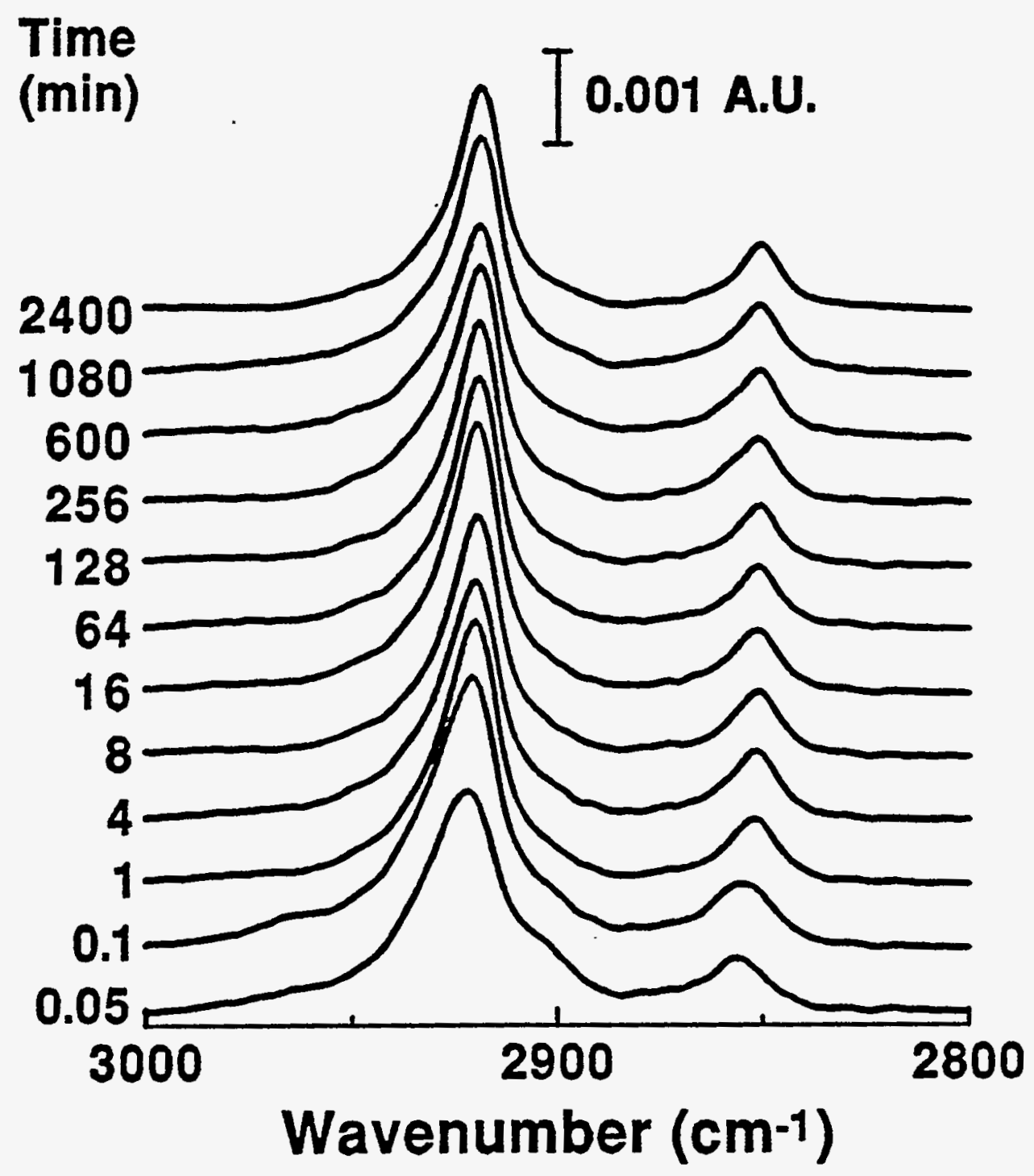

Figure 4. The IRRAS spectra in the high energy region of a MHA/Au monolayer as a function of immersion time. 


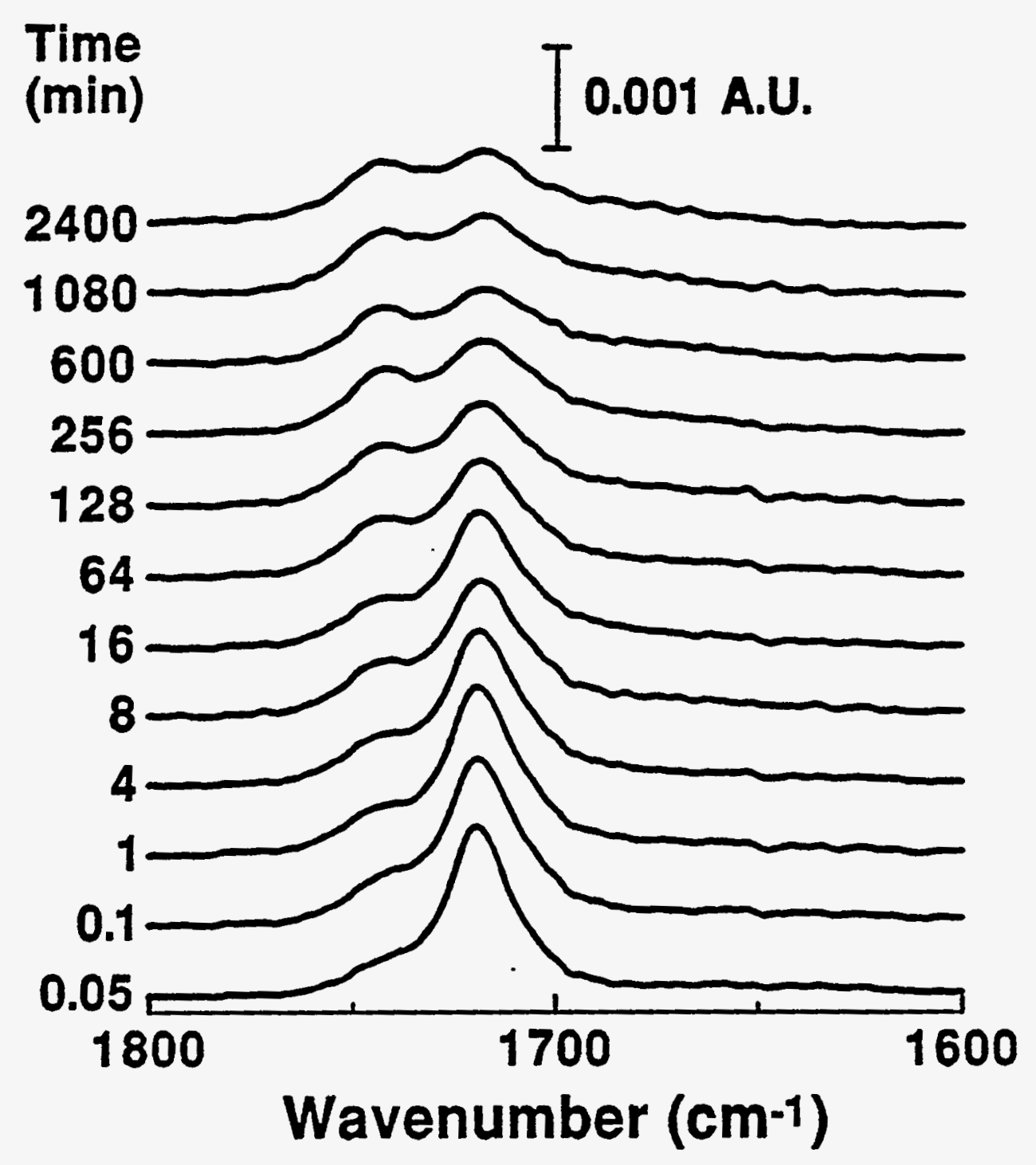

Figure 5. The IRRAS spectra in the $r(\mathrm{C}=0)$ region of a $\mathrm{MHA} / \mathrm{Au}$ monolayer as a function of immersion time. 


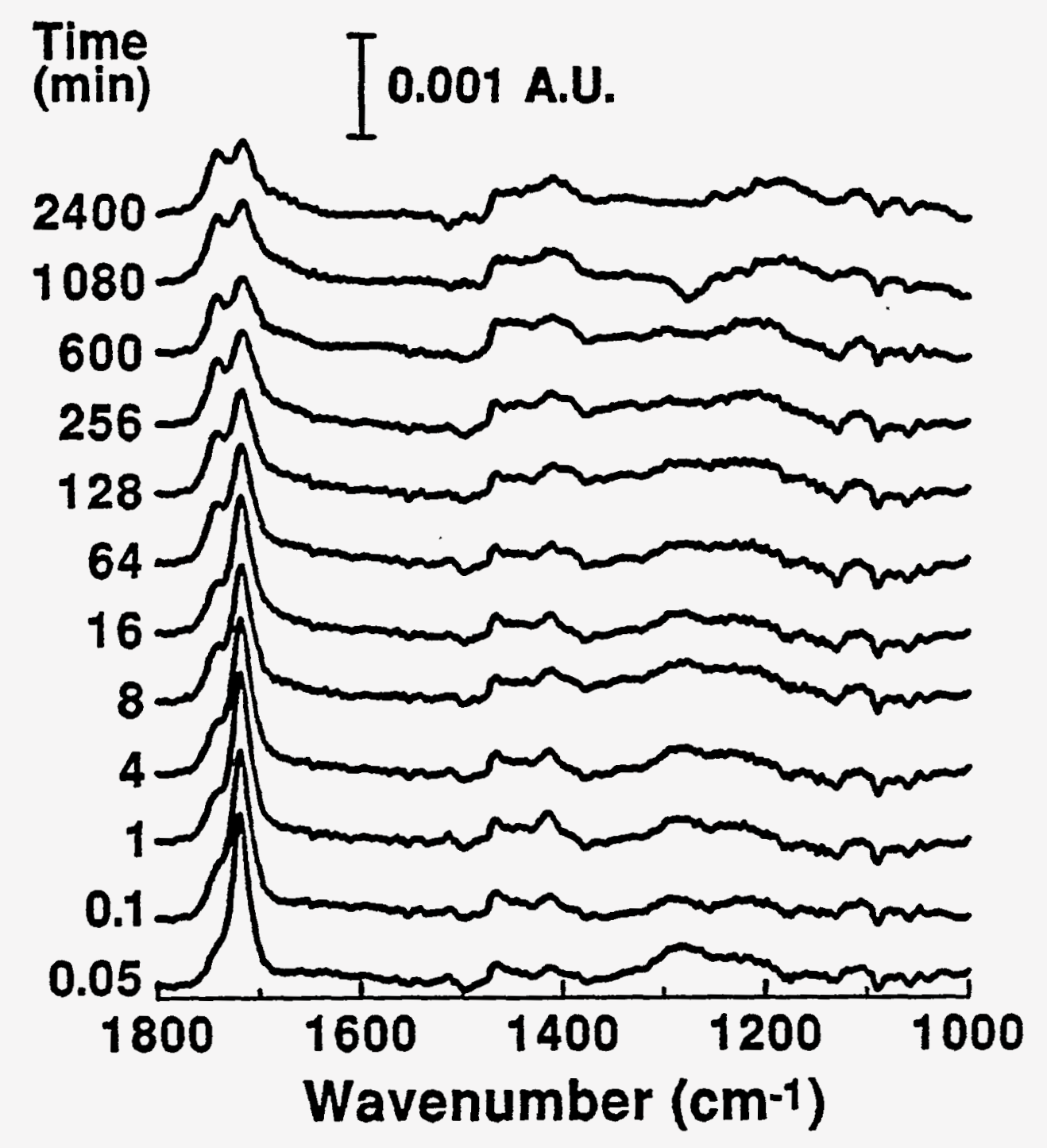

Figure 6. The IRRAS spectra in the low energy region of a MHA/Au monolayer as a function of immersion time. 


\section{IRRAS characterization}

Trends

It is possible to calculate the coverage, the packing density, and the tilt angle from IRRAS spectra assuming each molecule like its structure in $\mathrm{KBr}$ and is fully extended. The IRRAS spectra of a MHA/Au obtained as a function of immersion time in the high energy region and low energy region are shown in Figures 4 and 6, respectively. The vibrational mode assignments are summarized in Table 1.

The average tilt angle of the axis of a monolayer can be calculated from the following equation:

$$
\cos \theta_{m z}=\left(A_{o b s}\left(3 A_{c a l c}\right)\right.
$$

where $A_{o b s}$ and $A_{c a l c}$ are the observed and calculated peak area, $\theta_{m z}$ is the average tilt angle between the transition dipole moments $(m)$ and the surface normal $(z)^{25}$.

The calculated average tilt angles of this monolayer as a function of immersion time based on peak area are shown in Figure 7. At the early stage of the adsorption process, the large tilt angle implies that the packing density of the monolayer is low. This is consistent with the results from the thickness measurements. The tilt angle decreases to about $38^{\circ}$ in the first $16 \mathrm{~min}$ and there is little subsequent decrease. After $2400 \mathrm{~min}$ of immersion time, the tilt angle is $\sim 30^{\circ}$ which is in agreement with the $32^{\circ}$ determined by Nuzzo et al. ${ }^{19}$

At a tilt angle of $30^{\circ}$, this monolayer should have a packing density of $8.0 \times 10^{-10} \mathrm{~mol} / \mathrm{cm}^{2}$ (i.e. the distance between the nearest neighbor is $5.0 \AA$ which is the same as that for n-alkane thiolate monolayers on $\mathrm{Au}^{14}$.

The twist angle decreases from $57^{\circ}$ to $53^{\circ}$ in the first $16 \mathrm{~min}$ and thereafter decreases very slowly to a limiting value of $51^{\circ}$. This result indicates that the Au-S-C angle is $\sim 120^{\circ}$ regardless of the packing density or the tilt angle of the film ${ }^{19}$. 
Table 1. Peak positions, mode assignments, and transition dipole moment directions of $\mathrm{MHA} / \mathrm{Au}$ and a methyl-terminated thiolate monolayer on $\mathrm{Au}$.

$\mathrm{HO}_{2} \mathrm{C}\left(\mathrm{CH}_{2}\right)_{15} \mathrm{~S} / \mathrm{Au} \mathrm{CH}_{3}\left(\mathrm{CH}_{2}\right)_{13} \mathrm{~S} / \mathrm{Au}$

\begin{tabular}{|c|c|c|c|c|c|}
\hline Mode assignment & obs. & calc. & obs. & calc. & $\begin{array}{l}\text { direction of } \\
\text { transition dipole }\end{array}$ \\
\hline & & & 2964 & & $\begin{array}{l}\perp_{\text {to }} \mathrm{C}-\mathrm{CH}_{3} \text { bond in } \\
\text { C-C-Chain plane }\end{array}$ \\
\hline$v_{\mathrm{a}}\left(\mathrm{CH}_{3, \mathrm{op}}\right)$ & & & b & 2954 & $\begin{array}{l}\perp \text { to } \mathrm{C}-\mathrm{CH}_{3} \text { bond } \perp \\
\text { C-C-C chain plane }\end{array}$ \\
\hline$v_{\mathrm{s}}\left(\mathrm{CH}_{3}, \mathrm{FR}_{1}\right)^{\mathrm{c}}$ & & & 2936 & & \| to $\mathrm{C}-\mathrm{CH}_{3}$ bond \\
\hline$v_{\mathrm{a}}\left(\mathrm{CH}_{2}\right)$ & 2918 & 2920 & 2918 & 2920 & $\begin{array}{l}\perp \text { to } C-C-C \text { chain } \\
\text { plane }\end{array}$ \\
\hline$v_{\mathrm{S}}\left(\mathrm{CH}_{3}, \mathrm{FR}_{2}\right)^{\mathrm{c}}$ & & & 2878 & & $\|$ to $\mathrm{C}-\mathrm{CH}_{3}$ bond \\
\hline$v_{\mathrm{s}}\left(\mathrm{CH}_{2}\right)$ & 2850 & 2850 & 2850 & 2851 & $\begin{array}{l}\| \text { to } \mathrm{C}-\mathrm{C}-\mathrm{C} \text { plane } \\
\text { bisceting } \mathrm{H}-\mathrm{C}-\mathrm{H}\end{array}$ \\
\hline$\nvdash(C=0)$ & $\begin{array}{l}1743 \\
1717\end{array}$ & & & & $\|$ to $\mathrm{C}=\mathrm{O}$ bond \\
\hline & 1680 & 1699 & & & \\
\hline$\delta\left(\mathrm{CH}_{2}\right)$ & 1470 & 1472 & 1465 & 1467 & $\begin{array}{l}\text { in } \mathrm{HCH} \text { plane, } \\
\text { bisecting } \mathrm{HCH}\end{array}$ \\
\hline$\gamma(\mathrm{C}-\mathrm{O})+\delta(\mathrm{C}-\mathrm{O}-\mathrm{H})^{\mathrm{d}}$ & & 1431 & & & \\
\hline$\delta\left(\alpha-\mathrm{CH}_{2}\right)$ & & 1410 & & & \\
\hline$\delta\left(\mathrm{CH}_{3}\right)$ & & & 1382 & 1372 & $\|$ to $\mathrm{C}-\mathrm{CH}_{3}$ bond \\
\hline $\begin{array}{l}\mathrm{CH}_{2} \text { wags } \\
\text { and twists }\end{array}$ & $\begin{array}{l}1350- \\
1170\end{array}$ & & & & $\|$ to axis of chain \\
\hline
\end{tabular}


Table 1. (continued)

a Orientation of transition dipoles for $\mathrm{C}-\mathrm{H}$ modes assuming all-trans conformation (R. G. Snyder, J. Chem. Phys. 1965, 42, 1744.). bThe $v_{\mathrm{a}}\left(\mathrm{CH}_{3, \mathrm{op}}\right)$ mode was not observed in the monolayer spectrum. ${ }^{\mathrm{C}} \mathrm{The}$ fermiresonance couplet, $v_{\mathrm{s}}\left(\mathrm{CH}_{3}, \mathrm{FR}_{1}\right)$ and $v_{\mathrm{s}}\left(\mathrm{CH}_{3}, \mathrm{FR}_{2}\right)$, is designated by the subscripts 1 and 2 , whcih refer to the higher and lower energy components, respectively. $d$ This band involves both the stretching of the $\mathrm{C}-\mathrm{O}$ bond and the deformation of the C-O-H angle (D. Hadzi and M. Pintar, Spectrochim. Acta, $1958,12,162$.) 


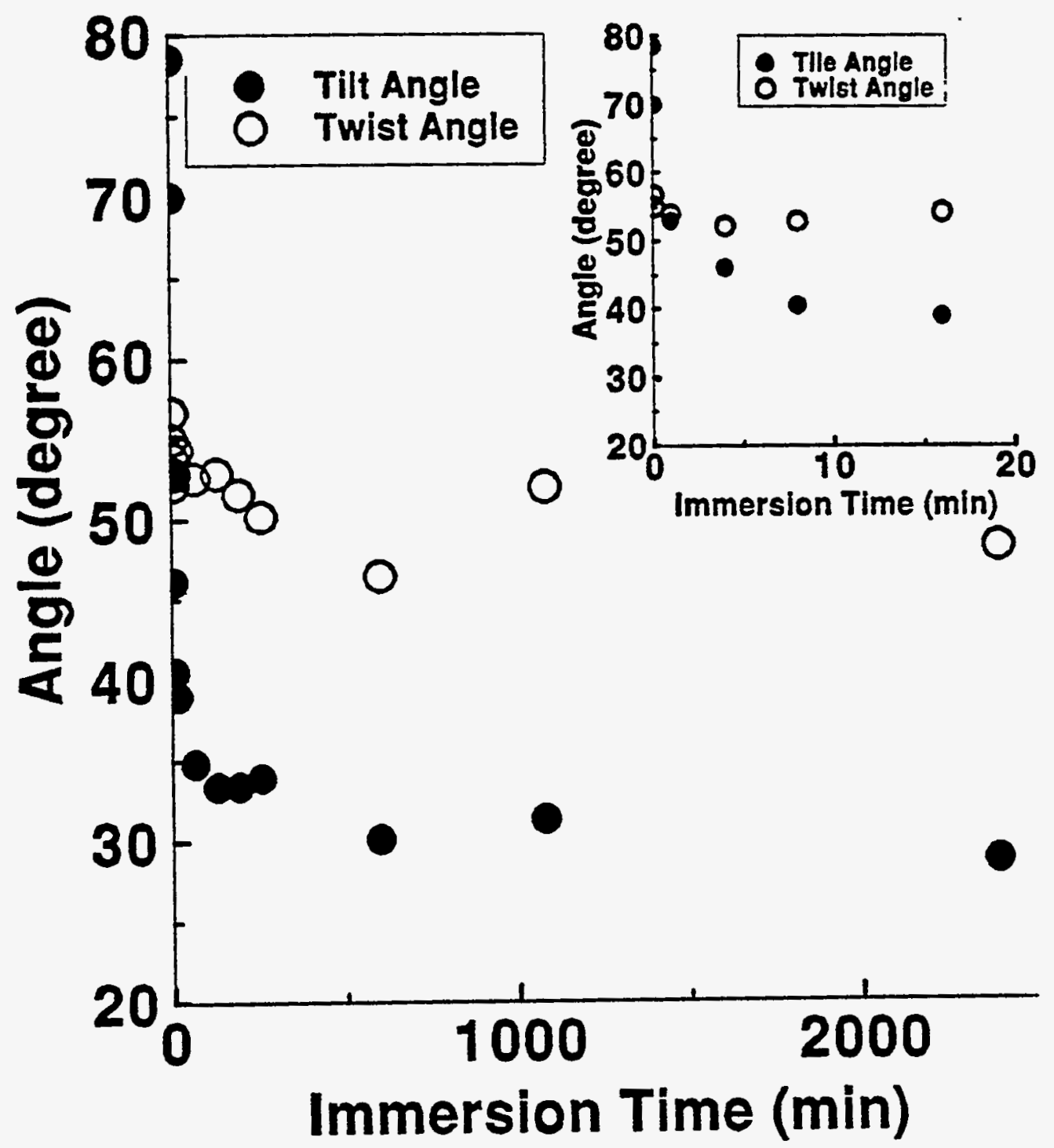

Figure 7. The claculated average tilt angle and twist angle of a MHA/Au as a function of immersion time. 


\section{Spectral deconvolution}

Methylene stretching region

Examination of the IRRAS spectra in more detail shows that there are at least five different $\mathrm{CH}_{2}$ features instead of the two features clearly evident in Figure 4. Five peaks yielded the best fit. The curve-fitted spectra and the experimental spectra for $1 \mathrm{~min}$ and $2400 \mathrm{~min}$ immersion time are shown in Figures 8 and 9. The main two peaks are the $v_{2}\left(\mathrm{CH}_{2}\right)$ at $2918 \mathrm{~cm}^{-1}$, and the $v_{\mathrm{s}}\left(\mathrm{CH}_{2}\right)$ at $2850 \mathrm{~cm}^{-1}$. These two peaks are indicative of crystalline-like packing of the methylene groups like that in solid crystal ${ }^{33}$.

In addition to these two peaks, there is the other set of peaks at 2928 $\mathrm{cm}^{-1}$ and $2858 \mathrm{~cm}^{-1}$ which are at higher energy than that of crystalline modes by $\sim 10 \mathrm{~cm}^{-1}$. These two peaks are the liquid-like $v_{\mathrm{a}}\left(\mathrm{CH}_{2}\right)$ and $v_{\mathrm{s}}\left(\mathrm{CH}_{2}\right)$ modes, respectively ${ }^{33}$.

Finally, there is one more peak at $2903 \mathrm{~cm}^{-1}$ which has not been classified in the literature yet. We believe that this peak is the $\mathrm{CH}_{2}$ stretching mode representing interactions of methylene groups with the gold surface, i.e. the C-H...Au agostic bond. (Details has been discussed in Paper II.) This peak is weak and finally disappears after $\sim 16 \mathrm{~min}$ of immersion. At very low coverage, almost all the molecules are laying down on the surface, as shown by the agostic $\mathrm{CH}_{2}$ mode at $2903 \mathrm{~cm}^{-1}$. When there is sufficient number of molecules to cover the surface area, some of the surface agostic molecules start to stand up to increase the packing density. As the coverage increases, the average tilt angle of the molecules decreases to make more room for more molecules to be absorbed until it reaches a minimum as shown in Figure 7.

The main four $\mathrm{CH}_{2}$ peaks as a function of immersion time are shown in Figure 10. Although the peak positions of both liquid-like $\gamma\left(\mathrm{CH}_{2}\right)$ modes change little, the peak positions of both crystalline-like $\left(\mathrm{CH}_{2}\right)$ modes decrease with immersion time, particularly in the first $\sim 30 \mathrm{~min}$, by more than two wavenumbers as shown in Figures 11 and 12, respectively. These data suggests that the packing of the polymethylene group becomes better and better with increasing the coverage of the monolayer.

In Figure 10, the peak areas of both crystalline-like $v\left(\mathrm{CH}_{2}\right)$ remain roughly constant while the areas of both liquid-like $\left(\mathrm{CH}_{2}\right)$ decrease with 


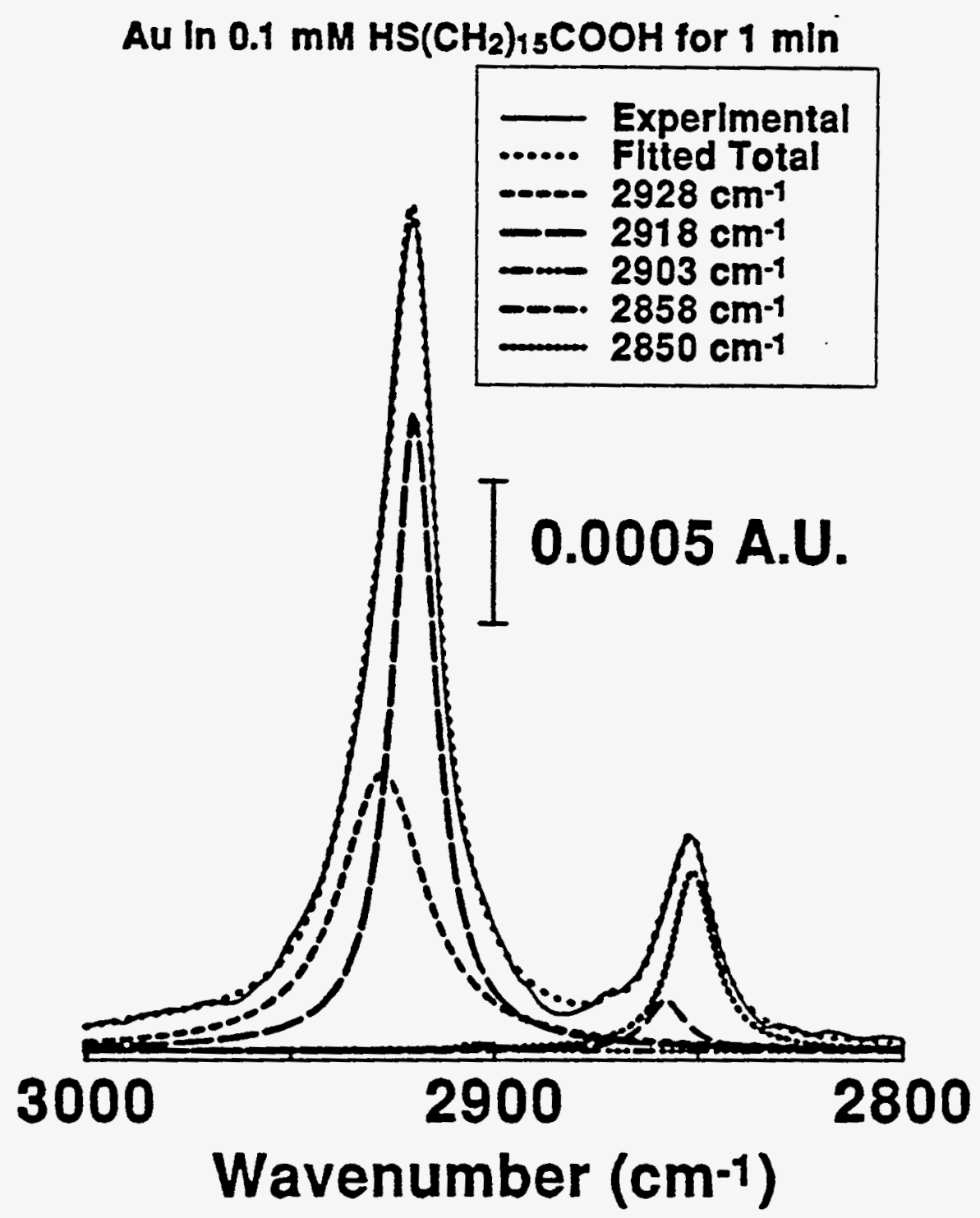

Figure 8. The curve-fitted spectra and the experimental spectrum in the $\checkmark\left(\mathrm{CH}_{2}\right)$ region for a MHA/Au in $0.1 \mathrm{mM}$ MHA solution for 1 $\min$. 
Au In $0.1 \mathrm{mM} \mathrm{HS}\left(\mathrm{CH}_{2}\right)_{15} \mathrm{COOH}$ for $2400 \mathrm{~min}$

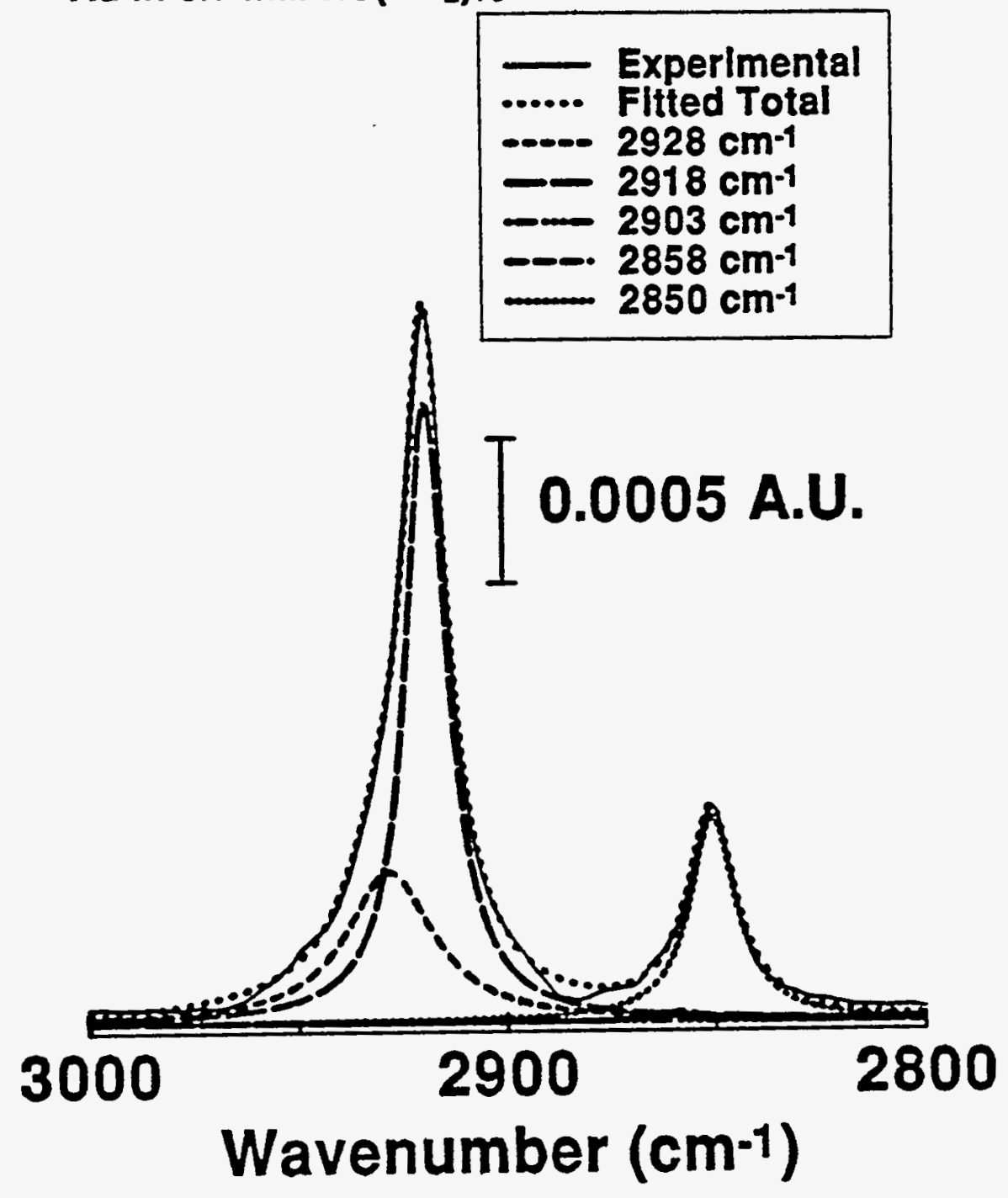

Figure 9. The curve-fitted spectra and the experimental spectrum in the $\checkmark\left(\mathrm{CH}_{2}\right)$ region for a MHA $/ \mathrm{Au}$ in $0.1 \mathrm{mM}$ MHA solution for 2400 $\min$. 


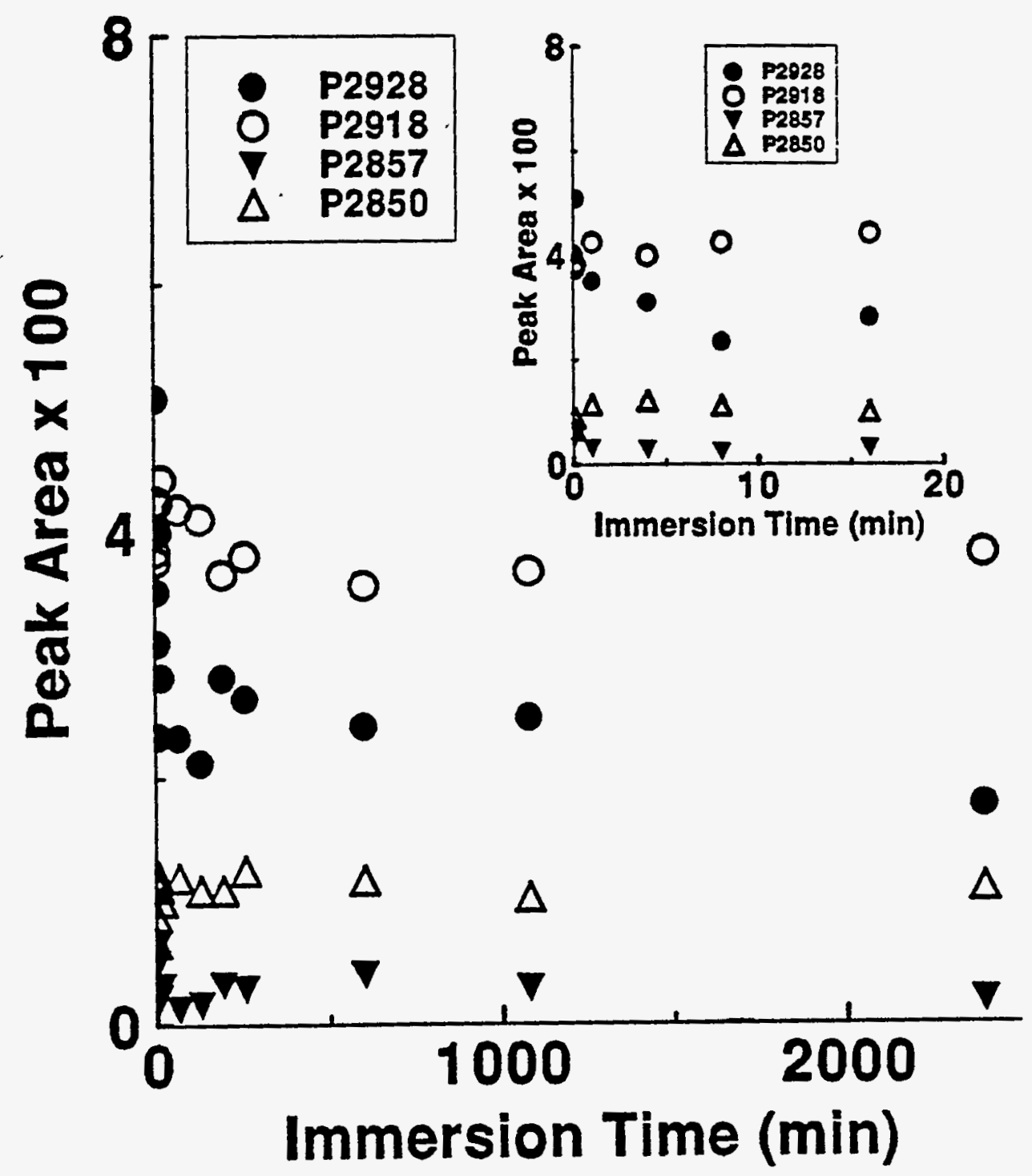

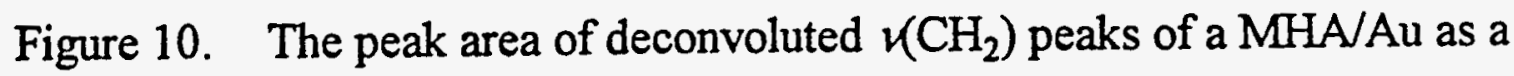
function of immersion time. 


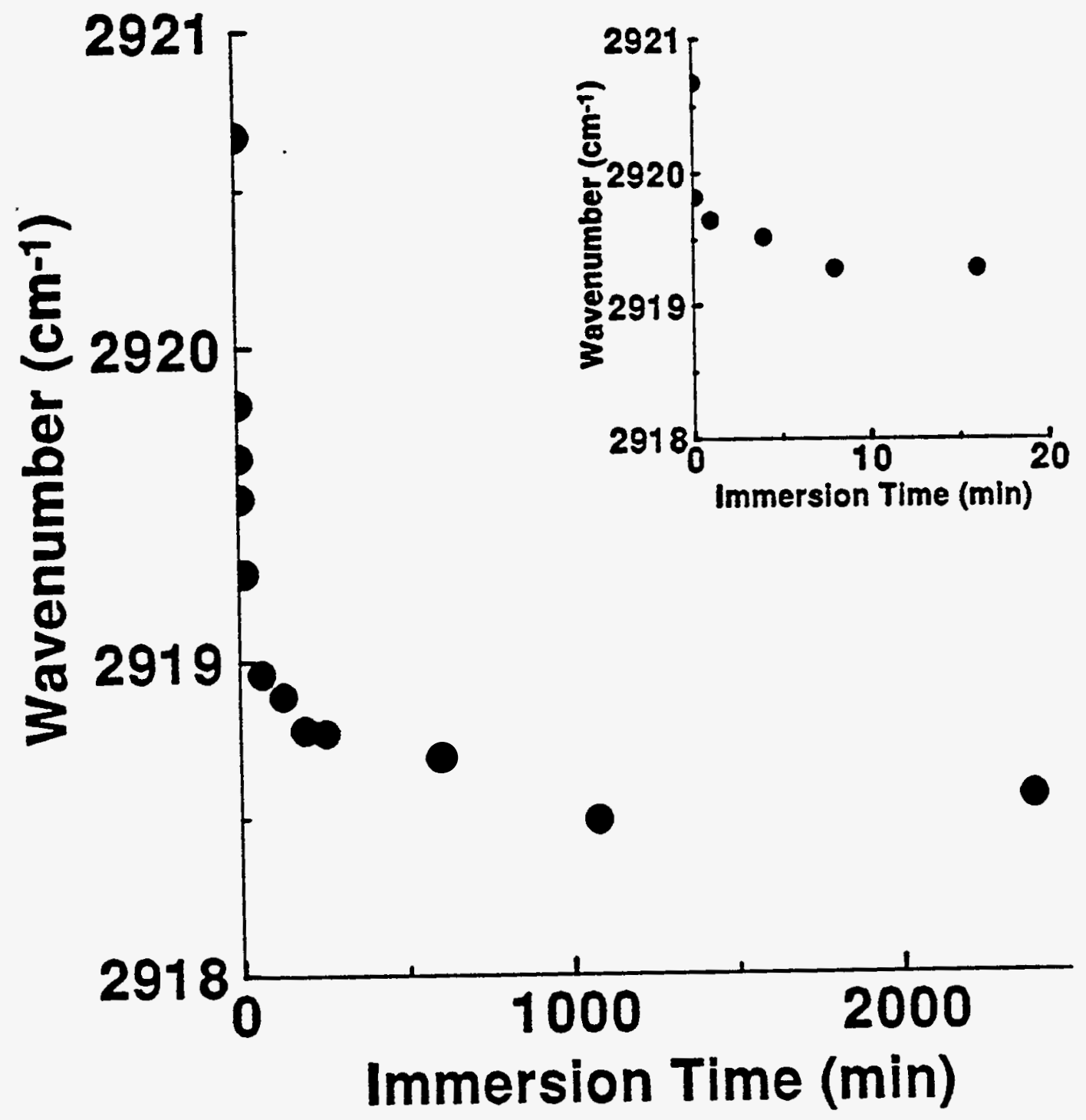

Figure 11. The peak position of the crystalline-like $v_{\mathrm{a}}\left(\mathrm{CH}_{2}\right)$ mode of a MHA/Au as a function of immersion time. 


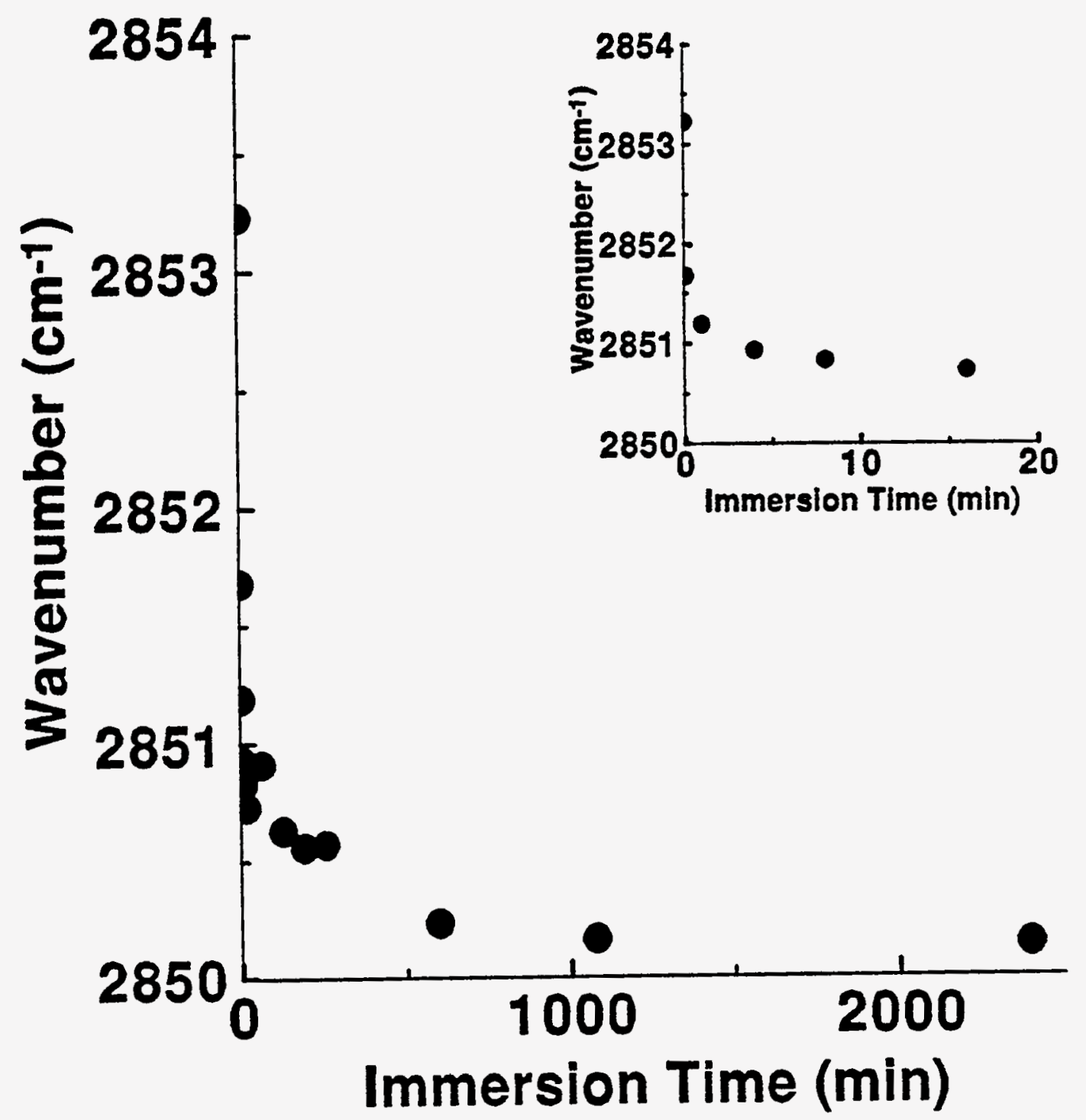

Figure 12. The peak position of the crystalline-like $v_{\mathrm{s}}\left(\mathrm{CH}_{2}\right)$ mode of a $\mathrm{MHA} / \mathrm{Au}$ as a function of immersion time. 
immersion time, especially during the first $30 \mathrm{~min}$. These data reveal that the amount of both the liquid-like $r\left(\mathrm{CH}_{2}\right)$ groups decrease substantially with increasing coverage while the amount of crystalline-like $r\left(\mathrm{CH}_{2}\right)$ groups only change slightly. These results further confirm that the MHA/Au monolayer structure is changing to maximize chain-chain interactions in order to stabilize the system with increasing coverage.

The percentage of this liquid-like $v_{\mathrm{a}}\left(\mathrm{CH}_{2}\right)$ decreases from $50 \%$ to $35 \%$ in the first $30 \mathrm{~min}$ and reaches $30 \%$ after $2400 \mathrm{~min}$. Although this monolayer packs very well after $30 \mathrm{~min}$, it has both trans and gauche $\mathrm{CH}_{2}$ segments at the end of the monolayer just like that in 1,32-dotriacontanedioic acid, $\mathrm{HO}_{2} \mathrm{C}\left(\mathrm{CH}_{2}\right)_{30} \mathrm{CO}_{2} \mathrm{H}$, adsorbed on $\mathrm{Ag}^{34}$. It is interesting that the full width at half maximum of the crystalline-like $v_{\mathrm{a}}\left(\mathrm{CH}_{2}\right)$ decreases from $\sim 16 \mathrm{~cm}^{-1}$ to $\sim 12$ $\mathrm{cm}^{-1}$ with increases in the coverage from $\sim 30 \%$ to $100 \%$. This result also suggests that the MHA/Au monolayer forms a more ordered film with increasing the coverage.

\section{Carbonyl stretching region}

The average tilt angle of carbonyl dipole

In Figure 5, the peaks between $1150 \mathrm{~cm}^{-1}$ and $1300 \mathrm{~cm}^{-1}$ are the progression peaks resulting from the presence of all-trans conformation sequences in the polymethylene chains of the monolayer ${ }^{19}$. Peaks between $1400 \mathrm{~cm}^{-1}$ and $1500 \mathrm{~cm}^{-1}$ are due to the $\mathrm{CH}_{2}$ bending mode. The peaks between $1600 \mathrm{~cm}^{-1}$ and $1750 \mathrm{~cm}^{-1}$ are the $r(\mathrm{C}=0)$ modes of carboxylic acid.

The experimental spectrum has all the peaks corresponding to the calculated spectrum. Interestingly, the experimental spectrum has one extra peak at $1743 \mathrm{~cm}^{-1}$, which is the non-hydrogen-bonded $\mathrm{r}(\mathrm{C}=\mathrm{O})$ mode.

The $r(\mathrm{C}=\mathrm{O})$ peak is shown as a function of immersion time as shown in Figure 6. As one can see, the peak iarea of the hydrogen-bonded dimeric peak decreases with increasing immersion time, while that of the nonhydrogen-bonded increases with time.

The average tilt angle for the $\mathrm{C}=\mathrm{O}$ dipole was calculated using equation 1 with a calculated IRRAS spectrum. The average tilt angles of $v(C=0)$ are plotted against immersion time as shown in Figure 13. The average tilt angle 


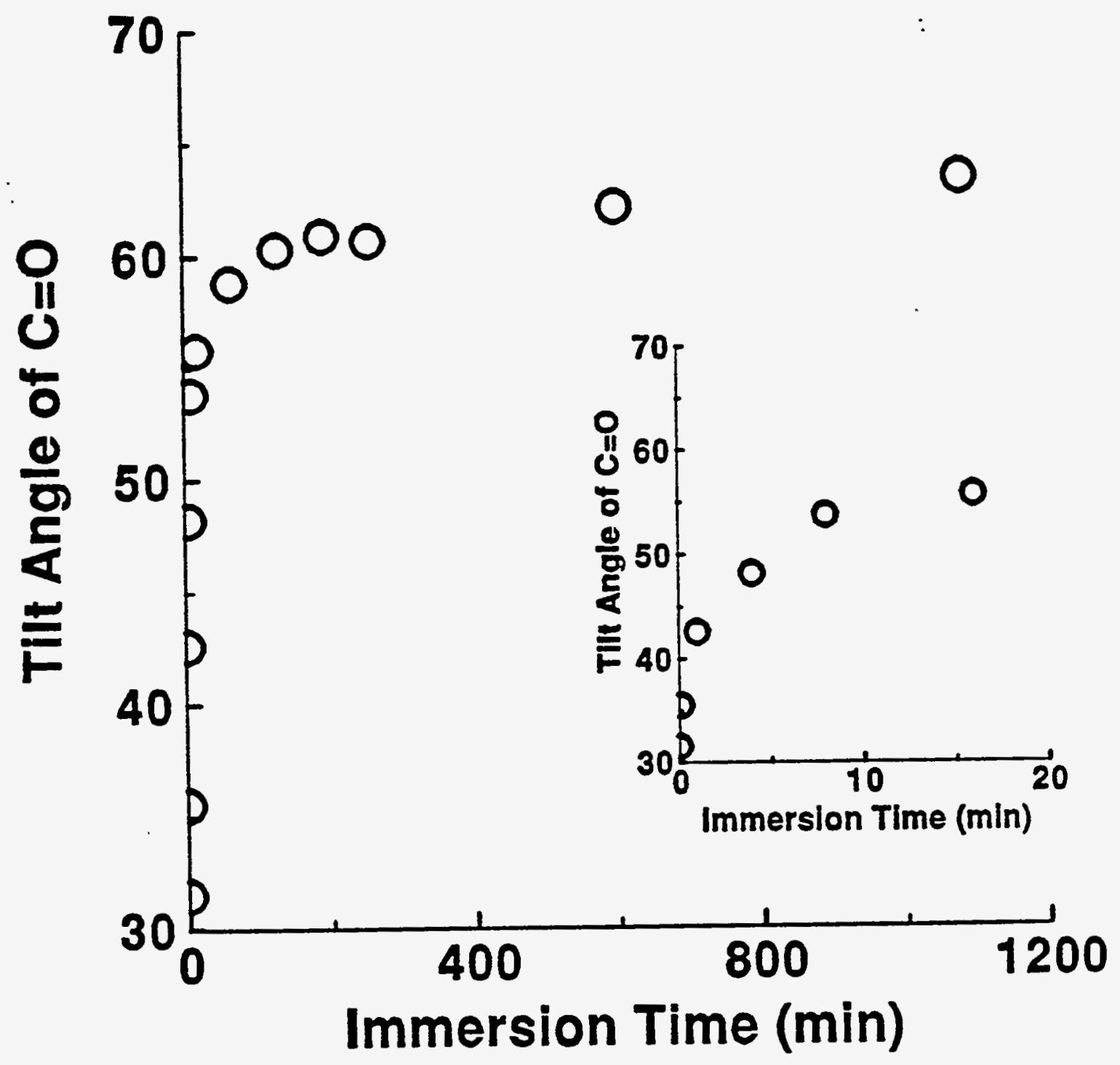

Figure 13. The average tilt angle of the $r(\mathrm{C}=0)$ diploe of a $M H A / A u$ as a function of immersion time. 
starts at $32^{\circ}$ at $0.10 \mathrm{~min}$ and increase sharply up to $62^{\circ}$ in $32 \mathrm{~min}$ and it remains virtually the same thereafter.

Three carbonyl modes

It is impossible to obtain a good fit for the $r(\mathrm{C}=0)$ envelope with only two peaks. The lower energy end cannot be fitted unless a third $\mathrm{C}=\mathrm{O}$ peak is introduced. These fitted curves and the experimental curves for monolayers with $1 \mathrm{~min}$ and $2400 \mathrm{~min}$ immersion time are plotted in Figures 14 and 15, respectively. In both cases, the fitted curves match the experimental curves well using three $v(\mathrm{C}=0)$ modes. There is a $r(\mathrm{C}=0)$ mode at $1680 \mathrm{~cm}^{-1}$ in addition to the non-hydrogen-bonded and the hydrogen-bonded dimeric $\checkmark(\mathrm{C}=0)$ modes. It is well known that the hydrogen bonding strengths the $v(\mathrm{C}=\mathrm{O})$ bond of the carboxylic acids and shifts the peak frequency of the $\checkmark(C=0)$ mode to lower energy by $20 \mathrm{~cm}^{-1}$ to $80 \mathrm{~cm}^{-1}$ depending upon the strength of the hydrogen bonding, i.e. the stronger the bonding the larger the $\operatorname{shift}^{35-40}$.

In this study, the non-hydrogen-bonded $\mathrm{r}(\mathrm{C}=\mathrm{O})$ is at $1743 \mathrm{~cm}^{-1}$, and the dimeric hydrogen-bonded $r(\mathrm{C}=\mathrm{O})$ is at $1717 \mathrm{~cm}^{-1}$. This hydrogen-bonded dimeric $\mathrm{C}=\mathrm{O}$ is believed to be the side-to-side hydrogen bonding instead of a head-to-head hydrogen bonding ${ }^{19}$.

The hydrogen-bonded polymeric $\mathrm{C}=\mathrm{O}$ mode

A comparison of the peak areas of all $v(C=0)$ peaks as a function of immersion time is shown in Figure 16. There is almost no non-hydrogenbonded $v(\mathrm{C}=0)$ peak in the beginning of the adsorption process when the coverage is very low, and the peak area of this peak increases with increasing immersion time and approaches a limiting value. On the other hand, that of the hydrogen-bonded dimeric $\checkmark(\mathrm{C}=\mathrm{O})$ decreases and approaches a limiting value with increasing immersion time as shown in Figure 16. The peak area of the low energy $r(\mathrm{C}=0)$ peak increases and approaches a limiting value with immersion time. It is very clear that most of the changes in peak area of these three peaks occurs during the first $60 \mathrm{~min}$ of immersion.

The decrease in the peak area is the combination result of the 
Au in $0.1 \mathrm{mM} \mathrm{HS}\left(\mathrm{CH}_{2}\right)_{15} \mathrm{COOH}$ for $1 \mathrm{~min}$

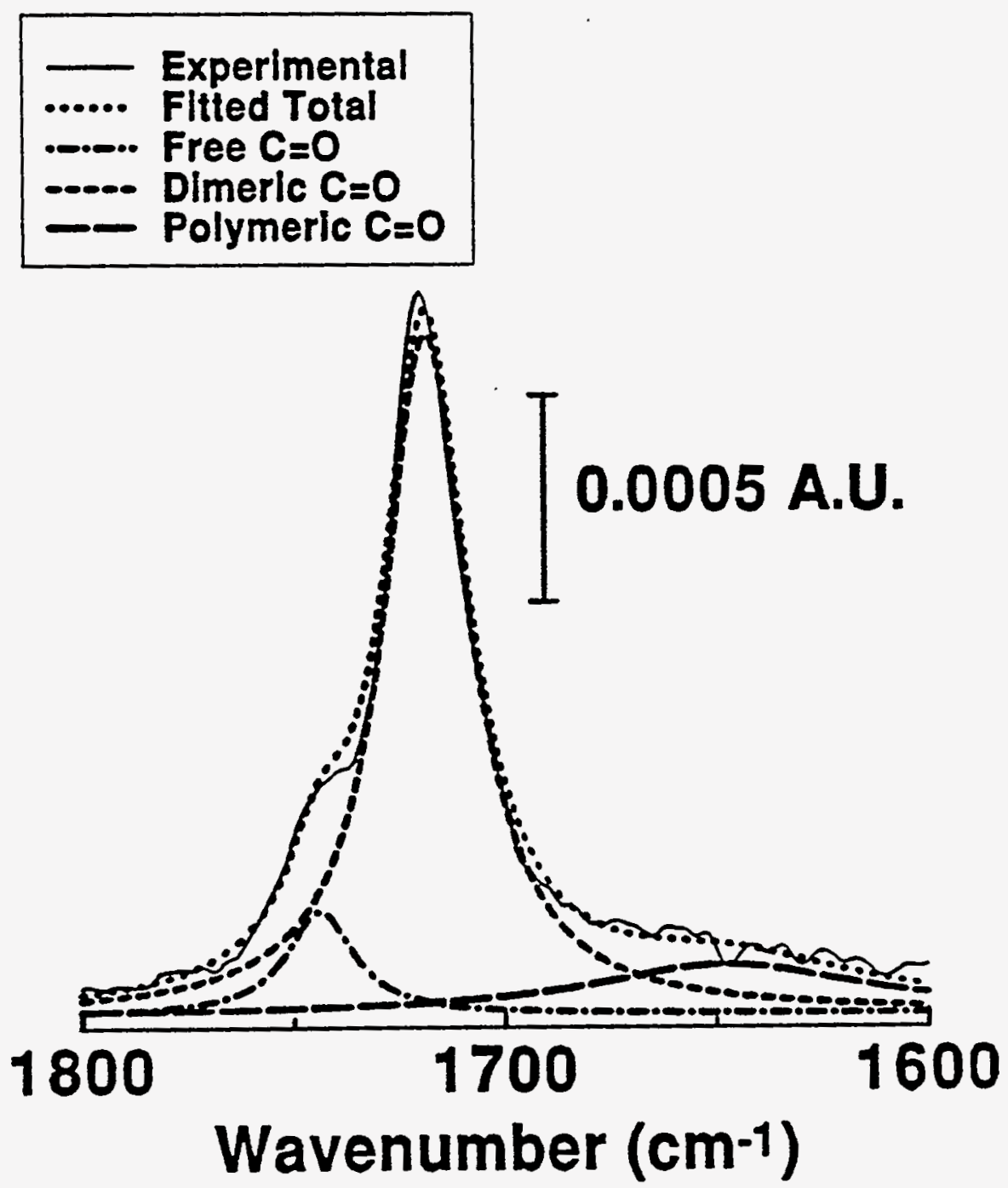

Figure 14. The curve-fitted spectra and the experimental spectrum in the $\checkmark(C=0)$ region for a $M H A / A u$ in $0.1 \mathrm{mM} M H A$ solution for 1 $\min$. 
Au in $0.1 \mathrm{mM} \mathrm{HS}\left(\mathrm{CH}_{2}\right)_{15} \mathrm{COOH}$ for $2400 \mathrm{~min}$
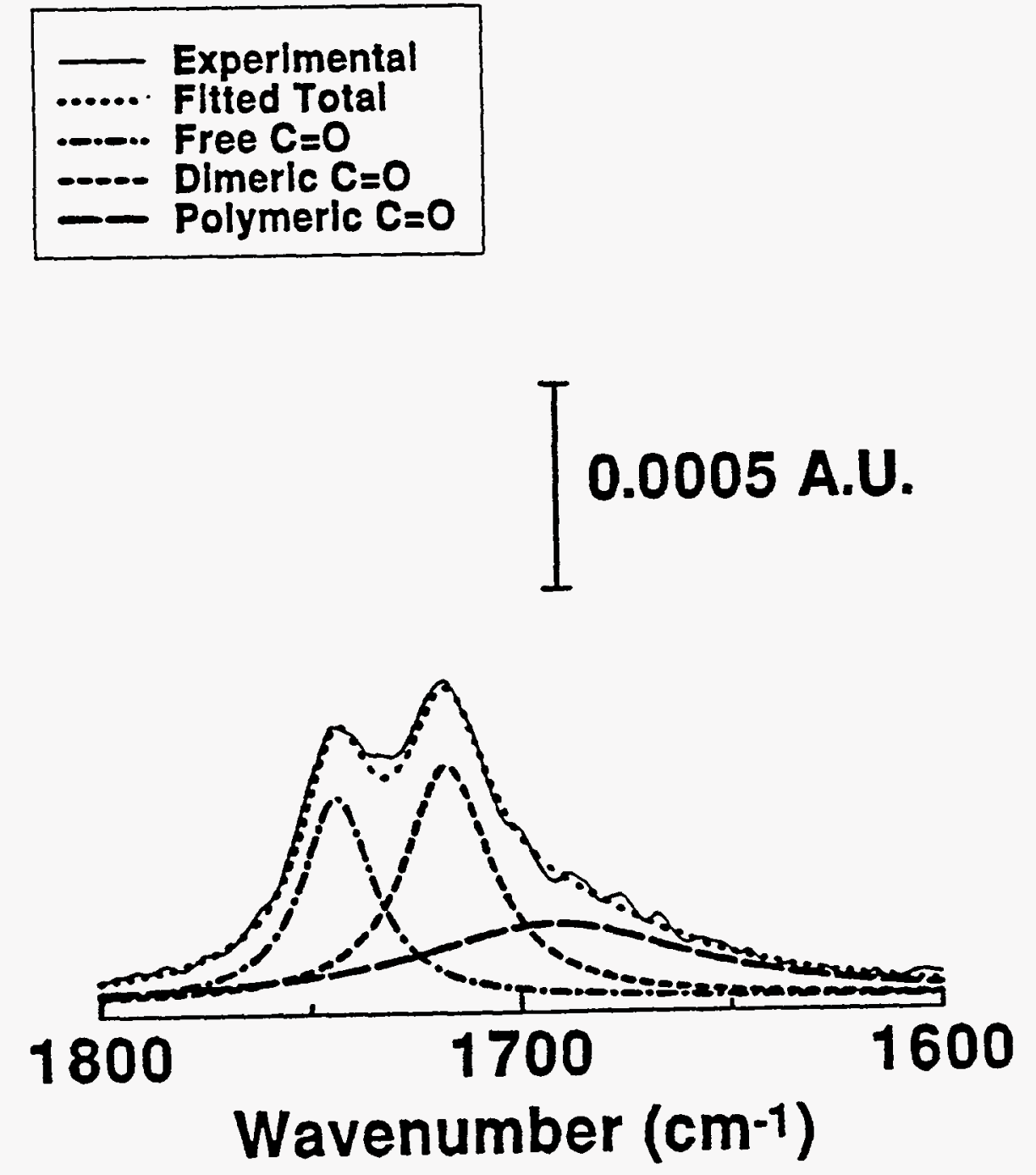

Figure 15. The curve-fitted spectra and the experimental spectrum in the $v(\mathrm{C}=\mathrm{O})$ region for a $\mathrm{MHA} / \mathrm{Au}$ in $0.1 \mathrm{mM} \mathrm{MHA}$ solution for $2400 \mathrm{~min}$. 


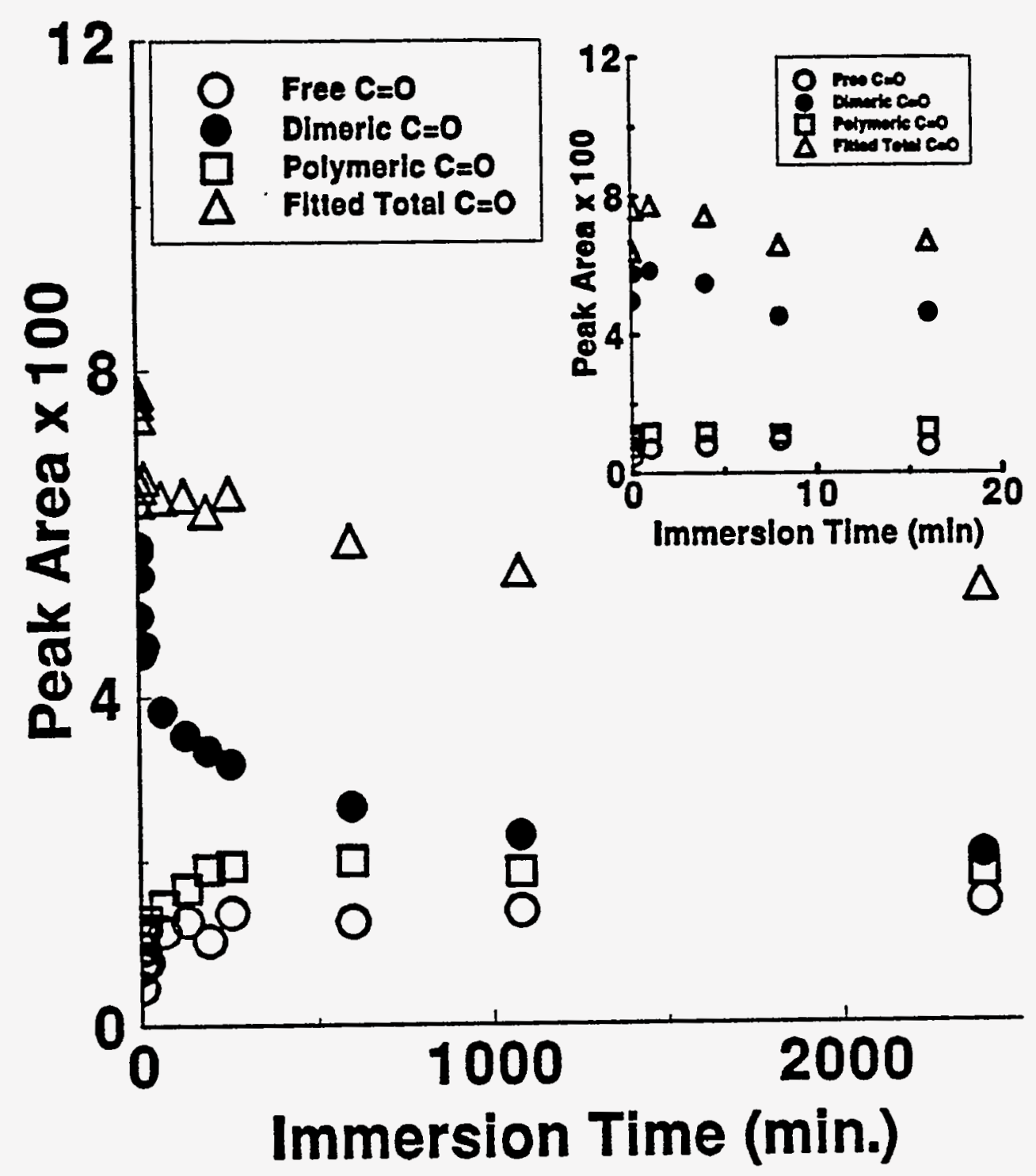

Figure 16. The peak area of deconvoluted $r(\mathrm{C}=0)$ peaks of a MHA/Au as a function of immersion time. 
increase of the coverage and the increase in the average tilt angle of $v(\mathrm{C}=\mathrm{O})$ dipole. The decrease in the peak area due to the increase in the average tilt angle is greater than the increase in the peak area due to the increase in the coverage of the monolayer. As a result, the total peak area of all three $\checkmark(\mathrm{C}=0)$ peaks decrease with increasing the coverage. If this is taken into account, the decrease in peak area of the hydrogen-bonded dimeric $v(\mathrm{C}=0)$ is almost equal to the total increase in peak area of the other two peaks particullary after $8 \mathrm{~min}$ of immersion. Furthermore, the peak area of the nonhydrogen-bonded $v(\mathrm{C}=0)$ peak is almost the same as that of the low energy $\checkmark(\mathrm{C}=\mathrm{O})$ peak along the way. Assuming the conservation of orientation, this result strongly suggests that there is a direct link between the hydrogenbonded dimeric $\gamma(\mathrm{C}=0)$ and the other two $\gamma(\mathrm{C}=0)$ peaks i.e. the hydrogenbonded dimeric $\checkmark(\mathrm{C}=\mathrm{O})$ mode is converted to both the non-hydrogen-bonded $\checkmark(\mathrm{C}=\mathrm{O})$ and low energy $r(\mathrm{C}=\mathrm{O})$ modes simultaneously. Therefore, the relative amounts of these three $r(\mathrm{C}=0)$ components is a function of coverage. More precisely speaking, the composition of these $\mathrm{v}(\mathrm{C}=0)$ peaks are controlled by the coverage of the monolayer.

In order to form a stable hydrogen bond for carboxylic acid, the distance between $\mathrm{O}$... O must be within 2.5 to $2.8 \AA$ range ${ }^{41}$. As the distance between the nearest $\mathrm{COOH}$ neighbor decreases from $7.0 \AA$ to $6.0 \AA$, the $\mathrm{COOH}$ groups start to form stronger and stronger hydrogen bonds.

Carboxylic acid groups still can form dimeric hydrogen bonds even though the distance is greater than $7.0 \AA$ by bending the polymethylene chain slightly. Based on these data, when the coverage is greater than $50 \%$ of the full monolayer, the distance between nearest $\mathrm{COOH}$ neighbors is less than $\sim 7.0 \AA$. (see below) In order for the $\mathrm{COOH}$ groups to form the most stable hydrogen bonds which is the polymeric hydrogen bonds the distance between the nearest $\mathrm{COOH}$ groups must be $\sim 6.0 \AA$. In order for some $\mathrm{COOH}$ to maintain this optimal distance to form polymeric hydrogen bonds in the confined space, some of the $\mathrm{COOH}$ groups must be squeezed at the same time so that the distance is too short to form any hydrogen bond at all. This structural change explains why the IRRAS spectra in Figure 16 show an increase in the amount of the hydrogen-bonded polymeric $v(\mathrm{C}=\mathrm{O})$ mode and also an increase in the 
amount of the non-hydrogen-bonded $v(\mathrm{C}=0)$ with increases in coverage simultaneously.

As coverage increases, some hydrogen-bonded dimeric $\mathrm{C}=\mathrm{O}$ groups form polymeric hydrogen bonds and force some to have no hydrogen bond at all.

\section{The PUAD/Au monolayer}

Finally, a monolayer of $\left(\mathrm{CH}_{3}\left(\mathrm{CH}_{2}\right)_{4}\right) \mathrm{SS}\left(\left(\mathrm{CH}_{2}\right)_{10} \mathrm{CO}_{2} \mathrm{H}\right) / \mathrm{Au}(\mathrm{PUAD} / \mathrm{Au})$ with a short alkyl chain and long carboxylic acid chain was examined. The combination of the short alkyl chain and long carboxylic acid chain prevents the carboxylic acid groups from forming hydrogen-bonded polymeric even at full coverage because one pair of carboxylic acid groups is separated from another by alkyl groups.

The spectra of the $v(\mathrm{C}=\mathrm{O})$ and the $\downarrow\left(\mathrm{CH}_{2}\right)$ modes of a PUAD/Au as a function of immersion time are shown in Figures 17 and 18. There are no noticeable changes observed in the spectra and, in particularly, there is virtually no non-hydrogen-bonded $\mathrm{C}=\mathrm{O}$ or hydrogen-bonded polymeric $\mathrm{C}=\mathrm{O}$ observed.

The reason why the hydrogen-bonded dimeric $\mathrm{C}=\mathrm{O}$ mode dominates is because the $\mathrm{COOH}$ groups are structurally impaired in forming any hydrogenbonded polymeric $\mathrm{C}=\mathrm{O}$ group or non-hydrogen-bonded $\mathrm{C}=\mathrm{O}$ group. The monolayer from the disulfide forms so that there is a $\mathrm{n}$-alkane segment in between two adjacent $\mathrm{C}=\mathrm{O}$ groups. Perhaps the reason why there is no change in $\mathrm{CH}_{2}$ stretching mode is that there is no room for $\mathrm{CH}_{2}$ to move around because the disulfide almost already forms a densely packed structure in less than $0.1 \mathrm{~min}$. Since the disulfide consists of a long carboxylic acid chain and a short alkyl chain, it is very unlikely that there will be a situation favorable for extensive Methylene chain-chain interaction between neighbors. The lack of abundant chain-chain interactions between the long carboxylic acid chains discourages these acid groups from forming any hydrogen-bonded polymeric $\mathrm{C}=\mathrm{O}$ bonds. As a consequence, the hydrogen-bonded dimeric is the dominant peak at all times regardless of either the immersion time or the coverage. 


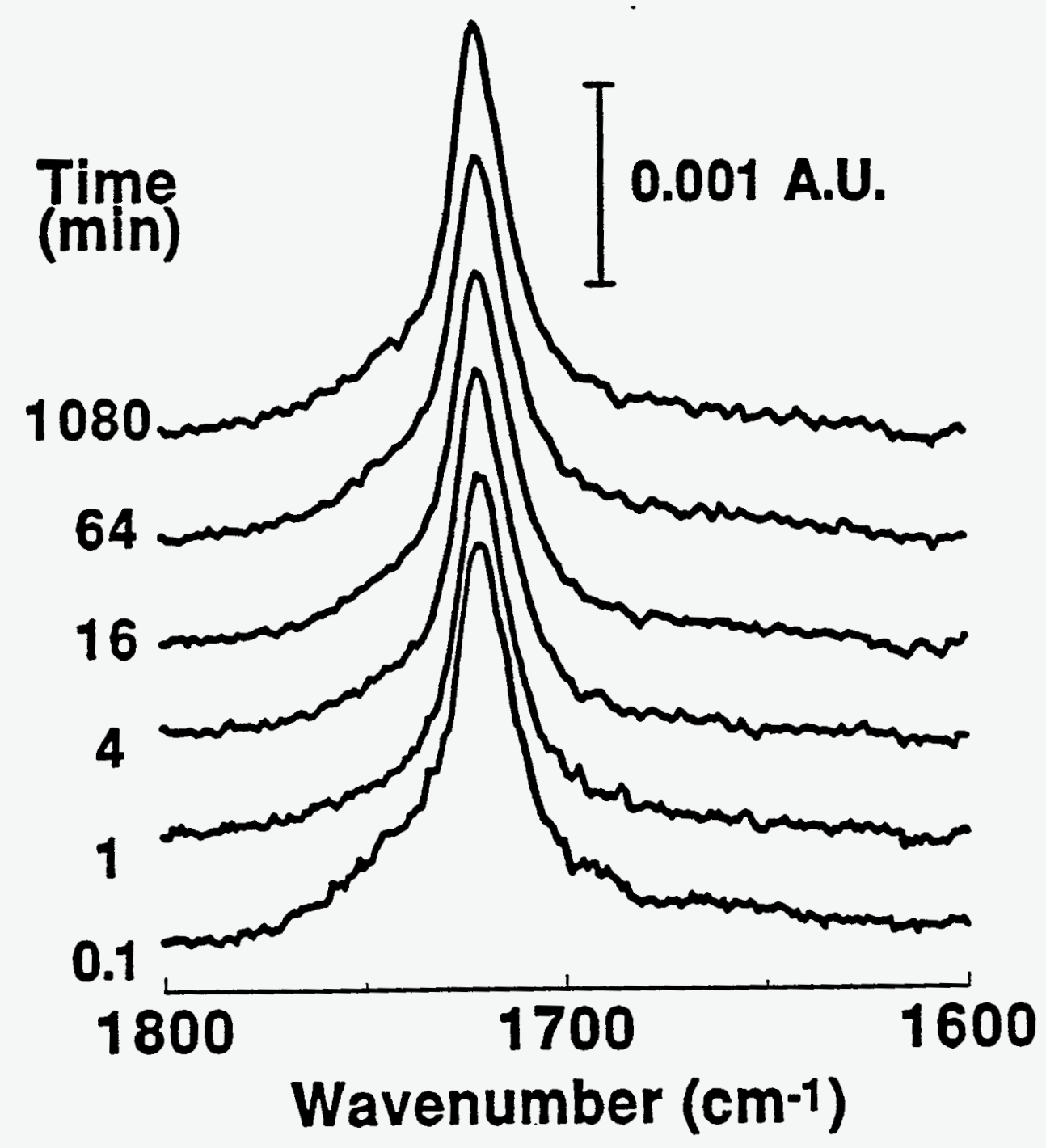

Figure 17. The IRRAS spectra in low energy region for a PUDA/Au as a function of immersion time. 


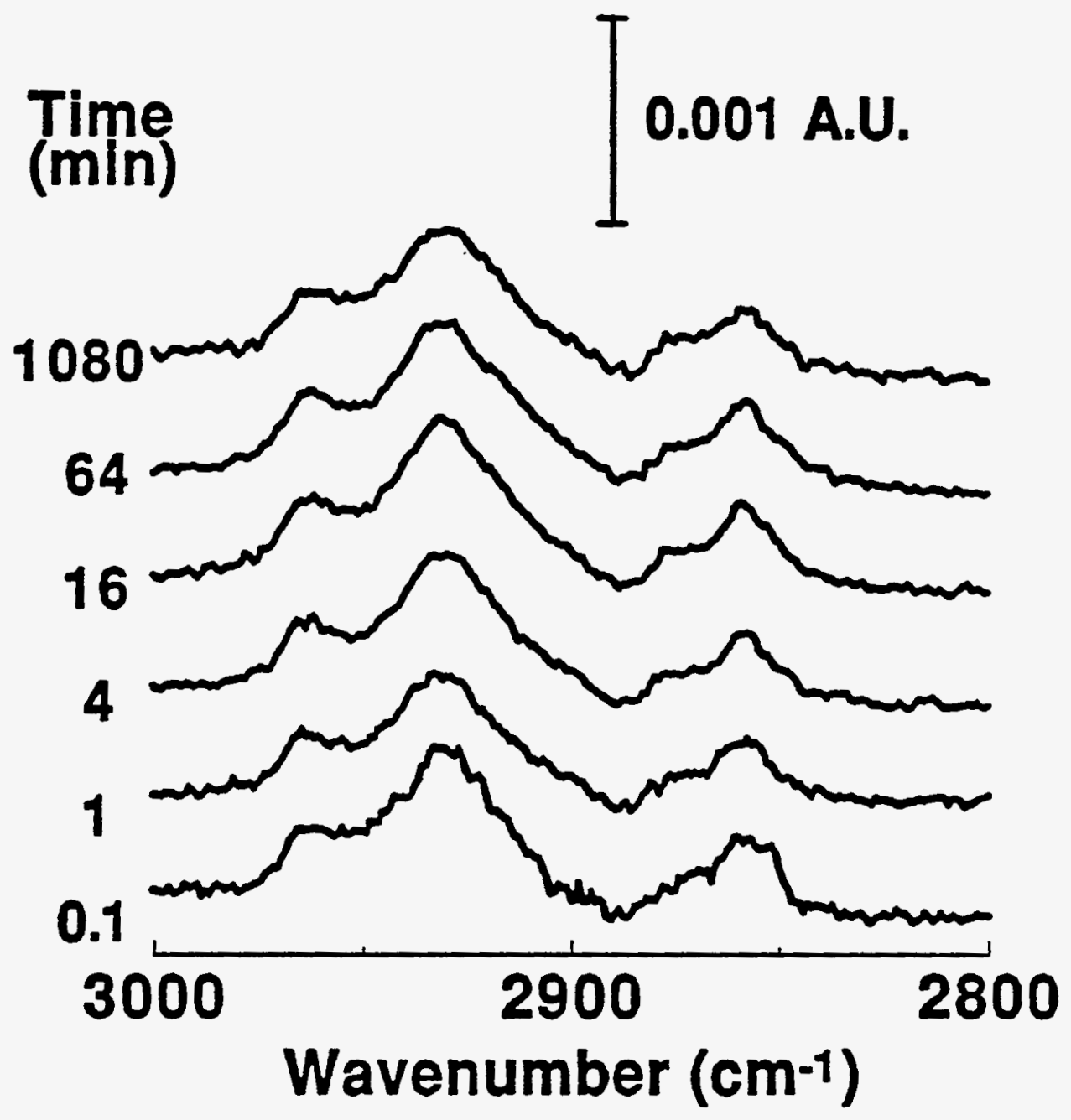

Figure 18. The IRRAS spectra in high energy region for a PUDA/Au as a function of immersion time. 
This data show that when the distance between the most adjacent carboxylic acid groups is greater than $7.0 \mathrm{~A}$, only hydrogen-bonded dimeric $\mathrm{C}=\mathrm{O}$ group exists without any hydrogen-bonded polymeric $\mathrm{C}=\mathrm{O}$ group or non-hydrogen-bonded $\mathrm{C}=\mathrm{O}$ group observed. Obviously, when there is no hydrogen-bonded polymeric $\mathrm{C}=\mathrm{O}$, there is no non-hydrogen-bonded $\mathrm{C}=\mathrm{O}$ either. This strongly suggests that the hydrogen-bonded polymeric $\mathrm{C}=\mathrm{O}$ and non- hydrogen-bonded $\mathrm{C}=\mathrm{O}$ are associated with each other. This finding is consistent with the result that shows the same increase in the amount of the peak area of both $\mathrm{C}=0$ peak with increasing immersion time.

All these evidences strongly confirm that the coverage-induced nonhydrogen-bonded $\mathrm{C}=\mathrm{O}$ and hydrogen-bonded polymeric $\mathrm{C}=\mathrm{O}$ modes evolve from the hydrogen-bonded dimeric $\mathrm{C}=\mathrm{O}$.

\section{Coverage dependence of $\mathrm{C}=\mathrm{O}$ groups}

To make sure that the change in $\mathrm{C}=\mathrm{O}$ structure is solely due to the change in the coverage but not because of immersion time, the solvent, or air, several more experiments were executed as following: after immersion in 0.10 $\mathrm{mM}$ solution for $1.0 \mathrm{~min}, \mathrm{MHA} / \mathrm{Au}$ monolayers were transferred and emersed in $0.1 \mathrm{M} \mathrm{HCl}$ ethanol solution, absolute ethanol, or air media for different time periods and then were rinsed successively with absolute ethanol and hexane, and dried under a stream of prepurified $\mathrm{N}_{2}$. The IRRAS spectra of the $\checkmark(C=0)$ modes of these samples are shown in Figures 19, 20, and 21, respectively.

Virtually no change in the $\mathrm{K}(\mathrm{C}=\mathrm{O})$ peaks with time in these three cases strongly suggests that the increase in the amount of both non-hydrogenbonded $v(\mathrm{C}=\mathrm{O})$ and hydrogen-bonded polymeric $v(\mathrm{C}=0)$ modes are not due to immersion time, the solvent, or the replacement of the incorporated solvent or lateral diffusion on the surface to reduce defects and enhance packing as proposed by Bain and Whitesides ${ }^{26}$.

Since the distance between the nearest $\mathrm{COOH}$ neighbor, $d$, is reciprocally proportional to the square root of the packing density of a monolayer, it can be calculated as following:

$$
d=5.0 \AA /\left(\Gamma / \Gamma_{\infty}\right)^{1 / 2}
$$




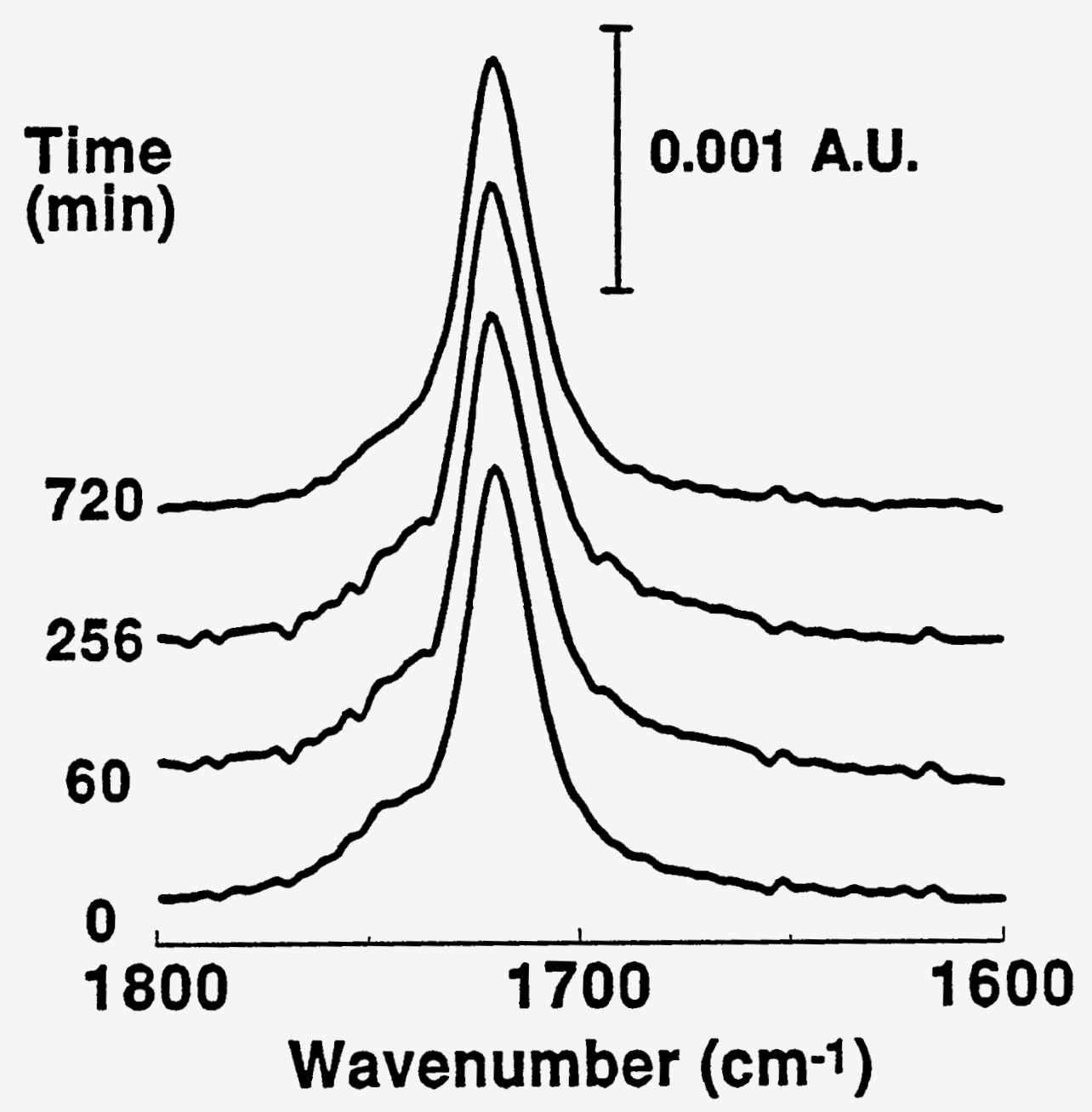

Figure 19. The IRRAS spectra in the low energy region for a MHA monolayer prepared in $0.1 \mathrm{mM}$ solution for $1 \mathrm{~min}$ and then transferred to $0: 1 \mathrm{M} \mathrm{HCl}$ solution as a function of immersion time. 


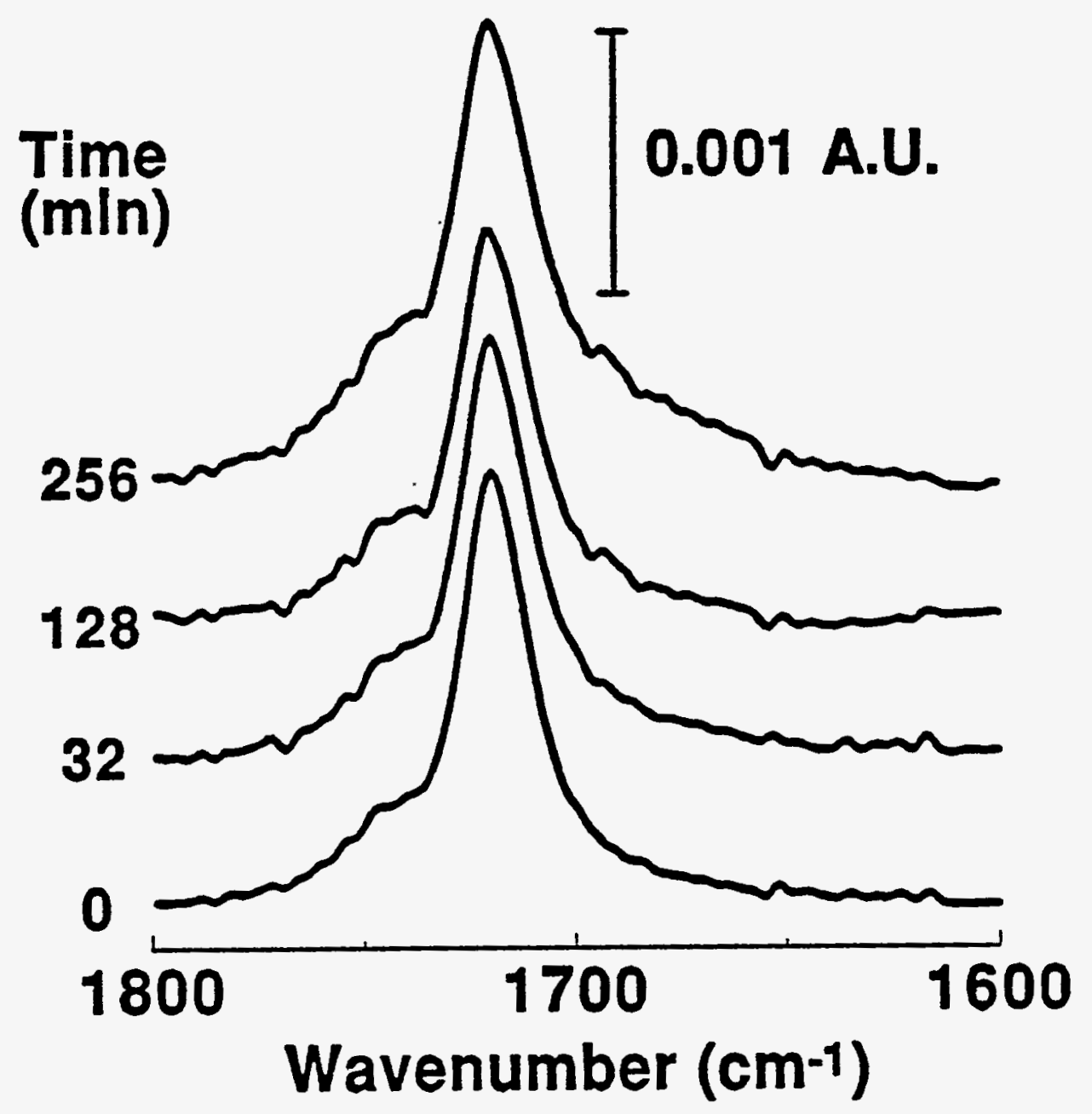

Figure 20. The IRRAS spectra in the low energy region for a MHA monolayer prepared in $0.1 \mathrm{mM}$ solution for $1 \mathrm{~min}$ and then transferred to pure ethanol solution as a function of immersion time. 


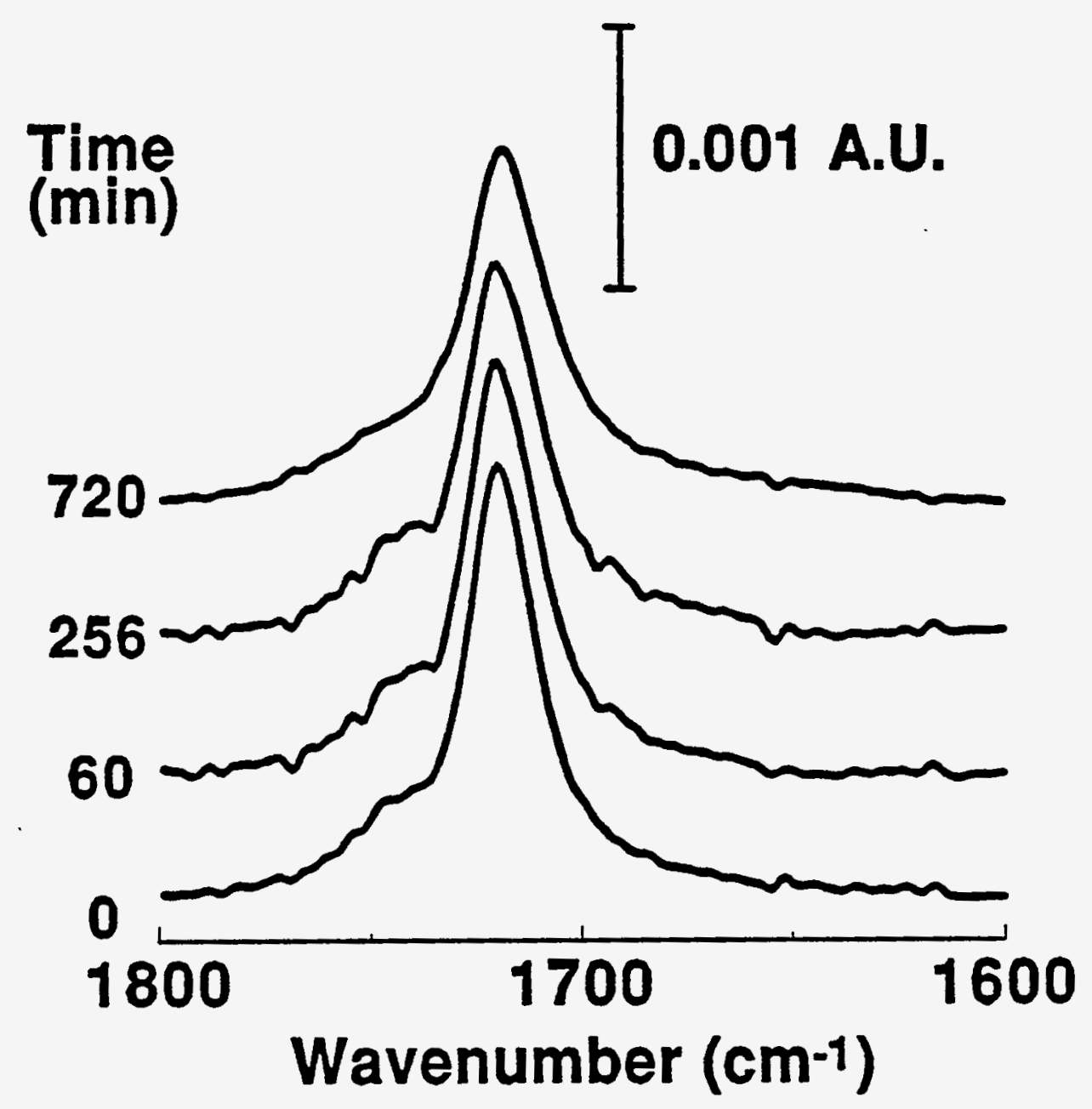

Figure 21. The IRRAS spectra in the low energy region for a MHA monolayer prepared in $0.1 \mathrm{mM}$ solution for $1 \mathrm{~min}$ and then exposed to air as a function of immersion time. 
where the $\Gamma$ and $\Gamma_{\infty}$ are the coverage of interest and the coverage of full coverage, respectively.

Since the coverage is proportional to the ellipsometric thickness equation 2 can be described as following:

$$
\mathrm{d}=5.0 \AA /\left(\mathrm{A} / \mathrm{A}_{\infty}\right)^{1 / 2}
$$

where $\mathrm{A}$ and $\mathrm{A}_{\infty}$ are the thickness of interest and the thickness at full coverage, respectively. In this study, $A_{\infty}$ is 22.0 at a full coverage of $8.0 \times 10^{-10} \mathrm{~mol} / \mathrm{cm}^{2}$.

The peak areas of these three $\{(\mathrm{C}=0)$ modes as a function of the distance, $d$, between the most adjacent carboxylic acid groups is shown in Figure 22. When the value of $d$ is greater than $7.0 \AA$, there is a little increase in the area of hydrogen-bonded dimeric $\mathrm{C}=0$ peak resulting from the substantial increases in coverage during the early stage of adsorption. It is clear that there is a remarkable decrease in the peak area of the hydrogenbonded dimeric $C=O$ peak when the value of $d$ is smaller than $7.0 \AA$. This decrease in peak area is accompanied by the increases in the peak areas of both free $\mathrm{C}=\mathrm{O}$ and hydrogen-bonded polymeric $\mathrm{C}=\mathrm{O}$ peaks especially when $\mathrm{d}$ approaches $5.0 \AA$. Therefore, we believe that the value of d plays a major role in determine the relative amount of these three distinct $\mathrm{C}=\mathrm{O}$ modes, i.e., when $\mathrm{d}>7.0 \AA, 7.0 \AA>d>5.0 \AA$, and $\mathrm{d}<5.0 \AA$, the majority carboxyl forms are the hydrogen-bonded dimeric $\mathrm{C}=\mathrm{O}$, the hydrogen-bonded polymeric $\mathrm{C}=\mathrm{O}$, and the non-hydrogen-bonded $\mathrm{C}=\mathrm{O}$, respectively. 


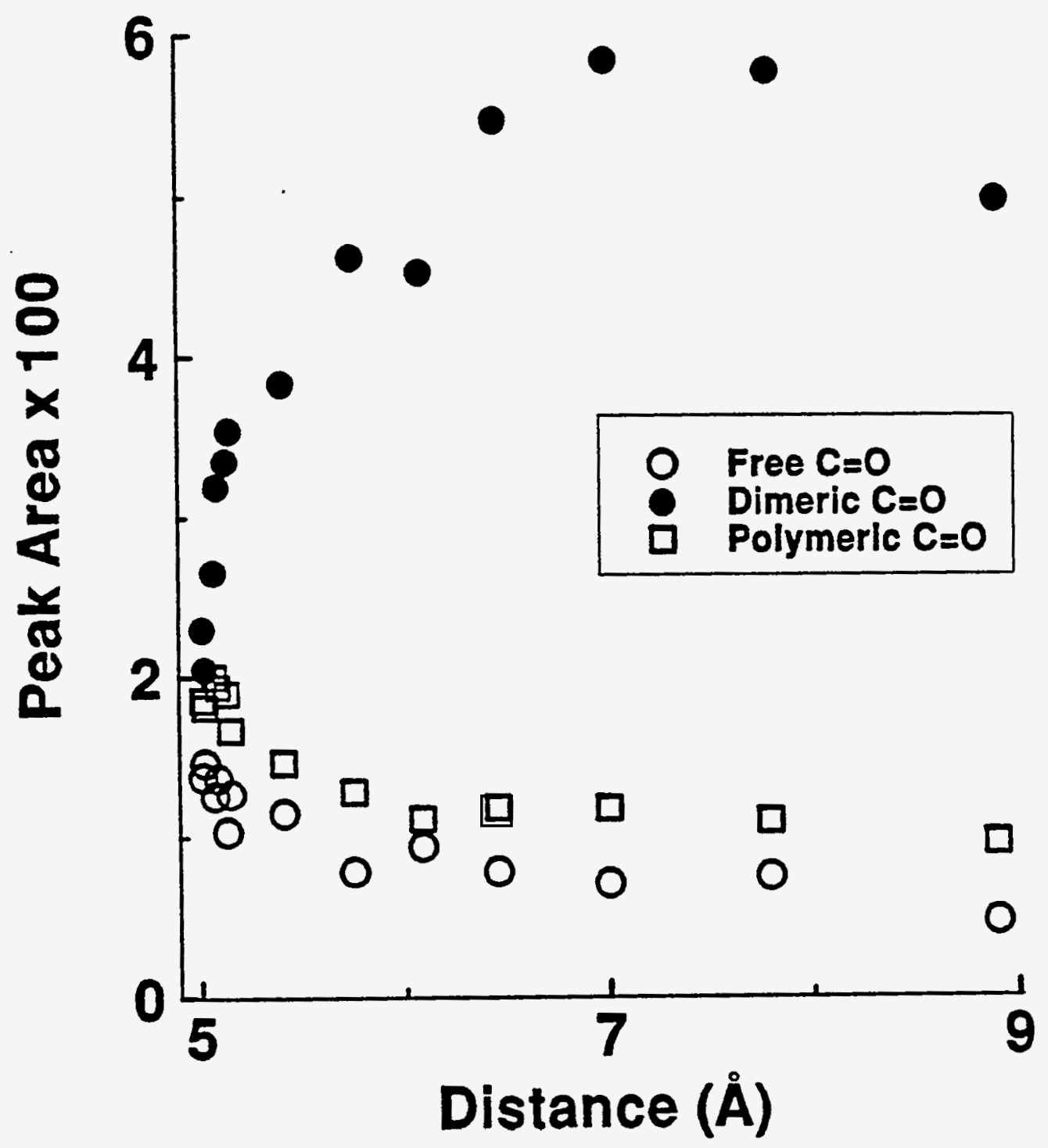

Figure 22. The peak areas of free $(C=0)$, hydrogen-bonded dimeric $\checkmark(\mathrm{C}=0)$, and hydrogen-bonded polymeric $\mathrm{r}(\mathrm{C}=0)$ as a function of the distance, $d$, between the most adjacent carbonylic acid groups. 


\section{CONCLUSIONS}

The adsorption process of the MHA on $\mathrm{Au}$ is a two-step process with a very fast reaction rate for the first step followed by a very slow reaction rate for the second step. It was determined that this slower reaction had little to do with the replacement of the incorporated solvent or lateral diffusion on the surface to reduce defects and enhance packing.

In addition to the crystalline-like asymmetric and $v_{\mathrm{s}}\left(\mathrm{CH}_{2}\right)$ peaks, there is one pair of the liquid-like $v_{\mathrm{a}}\left(\mathrm{CH}_{2}\right)$ and $v_{\mathrm{s}}\left(\mathrm{CH}_{2}\right)$ peaks which decreases with increasing immersion time. The FWHM of the crystalline-like $v_{\mathrm{a}}\left(\mathrm{CH}_{2}\right)$ peak becomes smaller with increases in the coverage. The tilt angle of this monolayer decreases from $\sim 78^{\circ}$ to $32^{\circ}$ in $\sim 32 \mathrm{~min}$ in $0.1 \mathrm{mM}$ solution and subsequently shows little change. On other hand, the twist angle remains almost the same with a value of $51^{\circ}$ regardless of the coverage.

The value of $\cos \theta_{\mathrm{a}}$ for $\mathrm{pH}=1.0 \mathrm{HCl}$ aqueous solution on a MHA/Au increases rapidly and almost linearly from $\sim 0.25$ to $\sim 0.95$ with increasing coverage from $0 \%$ to $75 \%$ of the full coverage. When the coverage is greater than $75 \%$, this $\cos \theta_{\mathrm{a}}$ value approaches a limiting value of 0.96 . It is believed that the surface of a MHA/Au is already completely covered by carboxylic acid groups when the coverage is greater than $75 \%$ of the full coverage.

There are three $v(\mathrm{C}=0)$ modes instead of only two. In addition to the non-hydrogen-bonded and hydrogen-bonded dimeric $\mathrm{r}(\mathrm{C}=0)$ peaks, there is the hydrogen-bonded polymeric $v(\mathrm{C}=0)$ bond at $1680 \mathrm{~cm}^{-1}$. At low coverage, the hydrogen-bonded dimeric $v(\mathrm{C}=0)$ mode is the only one observed. As the coverage increases, about $50 \%$ of this hydrogen-bonded dimeric $\mathrm{C}=\mathrm{O}$ mode splits into both non-hydrogen-bonded and hydrogen-bonded polymeric $\mathrm{C}=\mathrm{O}$ modes at the same time.

The average tilt angle of the $\mathrm{C}=\mathrm{O}$ dipole increases from $32^{\circ}$ to $62^{\circ}$ in order to form more stable side-to-side polymeric hydrogen bonds.

We believe that the three structures are a result of the distance, $d$. When $\mathrm{d}>7.0 \AA, 7.0 \AA>\mathrm{d}>5.0 \AA$, and $\mathrm{d}<5.0 \AA$, the majority carboxyl forms are the 
hydrogen-bonded dimeric $\mathrm{C}=\mathrm{O}$, the hydrogen-bonded polymeric $\mathrm{C}=\mathrm{O}$, and the non-hydrogen-bonded $\mathrm{C}=\mathrm{O}$, respectively. 


\section{REFERENCES}

(1) Nuzzo, R. G.; Korenic, E. M.; Dubois; L. H. J. Chem. Phys. 1990, 93, 767.

(2) Dubois, L. H.; Zegarski, B. R. J. Electron Spectrosc. Rel. Phenom. 1990, 54/55, 1143.

(3) Finklea, H. O.; Ravenscroft, M. S.; Snider, D. A. Langmuir 1993, 9, 223.

(4) Swalen, J. D.; Allara, D. L.; Andrade, J. D.; Chandross, E. A.; Garoff, S.; Israelachvili, J.; McCarthy, T. J.; Murray, R.; Pease, R. F.; Rabolt, J. F.; Wynne, K. J.; Yu, H. Langmuir 1987, 3, 932, and references therein.

(5) Bain, C. D.; Whitesides; G. M. Angew. Chem., Int. Ed. Engl. 1989, 28, 506 , and references therein.

(6) Whitesides, G. M.; Laibinis, P. E. Langmuir 1990, 6, 87, and references therein.

(7) Ulman, A An Introduction to Ultra-Thin Organic Films From Langmuir-Blodgett to Self-Assembly; Academic Press: San Diego, 1991.

(8) Seu, M.; Eisenberger, P.; McConnell, H. M. Proc. Natl. Acad. Sci. U. S. A. 1983, 80, 5795.

(9) Garoff, S.; Deckman, H. W.; Dunsmiur, J. H.; Alvarez, M. S.; Bloch, J. M. J. Phys. (Pairs) 1986, 47, 701.

(10) Meyer, E. Nature 1991, 349, 398.

(11) Porter, M. D.; Bright, T. B.; Allara, D. L.; Chidsey, C. E. D. J. Am. Chem. Soc. 1987, 109, 3559.

(12) Troughton, E. B.; Bain, C. D.; Whitesides, G. M.; Nuzzo, R. G.; Allara, D. L.; Porter, M. D. Langmuir 1988, 4, 365.

(13) Walczak, M. M.; Chung, C.; Stole, S. M.; Widrig, C. A.; Porter, M. D. J. Am. Chem. Soc. 1991, 113, 2370.

(14) Widrig, C. A.; Alves, C. A.; Porter, M. D. J. Am. Chem. Soc. 1991, 113, 2805.

(15) Alves, C. A.; Smith, E. L.; Porter, M. D. J. Am. Chem. Soc. 1992, 114, 1222. 
(16) Widrig, C. A.; Chung, C.; Porter, M. D. J. Electroanal. Chem. 1991, $310,335$.

(17) Strong, L.; Whitesides, G. M. Langmuir 1988, 4, 546.

(18) Dubois, L. H.; Zegarski, B. R.; Nuzzo, R. G. J. Phys. Chem. 1993, 97(8), 1665.

(19) Nuzzo, R. G.; Dubois, L. H.; Allara, D. L. J. Am. Chem. Soc. 1990, $112,558$.

(20) Widrig, C. A.; Alves, C. A.; Porter, M. D. J. Am. Chem. Soc. 1991, 113, 2805.

(21) Azzam, G. A.; Bashara, N. M. Ellipsometry and Polarized Light; Amsterdam: North-Holland Publishing, 1977.

(22) Bain, C. D.; Whitesides, G. M.; J. Am. Chem. Soc. 1989, 111, 7164.

(23) Handbook of Chemistry and Physics; Wsast, R. C.,Ed.; Chemical Rubber Co.: Boca Raton, FL, 1981.

(24) Ulman, A. J. Mater, Educ. 1989, 11, 205.

(25) Stole, S. M.; Porter, M. D. Apply. Spectrosc. 1990, 102, 981.

(26) Wilson, E. B., Jr.; Decius, J. C.; Cross, P. C. Molecular Vibrations; McGraw-Hill: New York, 1955; pp 285.

(27) Bain, C. D.; Troughton, E. B.; Tao, Y-T.; Evall, J.; Wihitesides, G. M.; Nuzzo, R. G. J. Am. Chem. Soc. 1989, 111, 321.

(28) Buck, M.; Eisert, F.; Fischer, J. Grunze, M.; Träger, F. Appl. Phys. 1991, $A 53,552$.

(25) Buck, M.; Eisert, F.; Fischer, J. Grunze, M.; Träger, F. J. Vac. Sci. Technol. 1992, A10, 926.

(30) Shimazu, K.; Yagi, I.; Uosaki, K. Langmuir 1992, 8, 1385.

(31) Thomas, R. C.; Sun, L.; Crooks, R. M.; Ricco, A. J. Langmuir 1991, 7, 620.

(32) Holmes-Farley, S. R.; Reamey, R. H.; McCarthy, T. J.; Deutch, J.; Whitesides, G. M. Langmuir 1985, 1, 725.

(33) Snyder, R. G.; Strauss, H. L.; Elliger, C. A. J. Phys. Chem. 1982, 86, 5145.

(34) Allara, D. L.; Atre, S. V.; Elliger, C. A.; Snyder, R. G. J. Am. Chem. Soc. 1991, 113, 1852.

(35) Gramstad, T.; Fuglevik, J. Spectrochim. Acta 1965, 21, 343. 
(36) Pullin, J. A.; Werner, J. Spectrochim. Acta 1965, 21, 1257.

(37) Figeys, H. P.; Nasielski, J. Spectrochim. Acta 1967, 23 A, 465.

(38) Bellamy, L. J.; Rogash, P. E. Spectrochim. Acta 1960, 16, 30.

(39) Collins, A. J.; Morgan, K. J. J. Chem. Soc. 1963, 3437.

(40) Brooks, C. J. W. J. Chem. Soc. 1960, 661.

(41) Jones, R. E.; Templeton, D. H. Acta Cryst. 1958, 11, 484. 


\section{PAPER IV}

INFRARED REFLECTION ABSORPTION SPECTROSCOPIC STUDIES OF THE ACID-BASE REACTIVITY OF ORGANIC MONOLAYER FILMS:

EMERSED CARBOXYLIC ACID-TERMINATED MONOLAYERS 


\section{INTRODUCTION}

Monolayer films prepared by the adsorption of thiol-containing compounds $\left(\mathrm{X}\left(\mathrm{CH}_{2}\right)_{n} \mathrm{SH}\right)$ at gold provide unique opportunities for the construction of organic interfaces with a well-defined composition and thickness $^{1-3}$. These films form as gold(I) thiolates ${ }^{4-8}$ to yield denselypacked structures for end groups that are of a size comparable with the polymethylene chain. In such cases, the spatial arrangement of the layer reflects the bonding of the thiolate head group with the underlying gold lattice. For alkanethiolates, both diffraction ${ }^{9,10}$ and direct atomic-scale imaging $^{11,12}$ techniques have established that the layers adopt a $(\sqrt{3} \times \sqrt{3}$ ) $\mathrm{R} 30^{\circ}$ overlayer structure at $\mathrm{Au}(111)$ (the predominant crystal face of the surface of evaporated gold films ${ }^{13-18}$ ). Because of these characteristics, thiolates at gold have proven extremely attractive in studies aimed at delineating the structural basis of processes occurring at solid-aqueous phase interfaces, e.g. electron-transfer ${ }^{19-23}$, wetting ${ }^{14,24-25}$, and bioadhesion $^{26,27}$.

One of the most important issues of these monolayers is their surface properties which are mainly affected by the end group of these monolayers ${ }^{28}$. Therefore, it is very important to understand and study the end group chemistry. The chemistry of the carboxylic acid end group of these carboxylic acid-terminated alkanethiolates is the target of this study.

Central to the above efforts is the need for detailed descriptions of the interactions between the adsorbed layer and the contacting aqueous phase. It is therefore of fundamental importance to develop techniques for the in situ characterization of such interfaces at a molecular level. For electrochemically-driven molecular transformations ${ }^{29,30}$, infrared reflection-absorption spectroscopy (IRRAS), coupled with electrochemical ${ }^{31-33}$ or optical ${ }^{34,35}$ modulation techniques, has proven particularly useful. However, as recently discussed, the properties of most thiolate monolayers limit the applicability of the noted modulation techniques ${ }^{36}$. Electrochemical modulation schemes are ineffective because the structure of these densely-packed monolayers is insensitive to applied voltages up to the onset of oxidative or reductive desorption ${ }^{37}$. 
Optical modulation techniques suffer from overlap of diagnostic spectral features (e.g. C-H stretching modes) of these monolayer and the $\mathrm{O}-\mathrm{H}$ stretching mode of aqueous solutions, leading to band shape and intensity distortions that complicate a structural interpretation ${ }^{36}$.

We recently described an approach that reduced the complications of obtaining IRRAS spectra in the $\mathrm{C}-\mathrm{H}$ stretching region of monolayer films under a thin layer of a contacting aqueous solution ${ }^{36,38}$. The approach is based on a cell design that facilitates the interchange of sample and reference substrates while maintaining a solution layer of effectively constant thickness. However, during studies of monolayers composed of various end-group functionalities ${ }^{36}$, we found characterizations of the reactivity of carboxylic acid-terminated thiolate monolayers to be particularly problematic using our approach. The principal complication arises from the proximity of the $\mathrm{O}-\mathrm{H}$ bending mode of aqueous solutions and the $v(C=O)$ of these layers, which reveals that we will need to gain further control in the repositioning of sample and reference substrates for such a measurement scheme to function effectively. A flow cell may work and we are pursuing such a possibility.

Interestingly, recent reports suggest another possibility for examining the reactivity of these layers under a thin aqueous solution layer ${ }^{38}$. This approach uses substrates that have been carefully emeresed from the contacting liquid. Early work with this approach has focused on examining the ionic structure at the electrode surface ${ }^{39-42}$, the contention being that such a structure remains intact upon removal of the electrode from solution. Though questions concerning the maintenance of the molecular structure of the interface upon introduction into an ultra high vacuum environment are not yet fully resolved ${ }^{43,44}$, recent Raman spectroscopic studies of adsorbed organic films have established that the molecular structure in solution is retained at electrodes immersed into environments at atmospheric pressure ${ }^{45}$. 


\section{EXPERIMENTAL SECTION}

\section{Substrate preparation}

Electrodes were prepared by the resistive evaporation of $15-20 \mathrm{~nm}$ of chromium, followed by 200-300 nm of gold, onto 3 inch by 1 inch glass substrates. The temperature during the evaporation, measured at the plate supporting the substrates, increased to $\sim 50^{\circ} \mathrm{C}$ as a result of radiative heating by the evaporation source. The evaporation rates were 0.2 and $2.0 \mathrm{~nm} / \mathrm{s}$ for chromium and gold, respectively. The pressure in a cryopumped E360A Edwards Coating System during evaporation was $<9 \times 10^{-5} \mathrm{~Pa}$. After $\sim 45 \mathrm{~min}$ cooling time, the evaporator was back-filled with purified $\mathrm{N}_{2}$. The substrates were then removed, analyzed by optical ellipsometry for the determination of the optical functions of the uncoated substrates, and immersed in the thiolcontaining solution.

The surfaces of the uncoated gold electrodes have been characterized previously at both a macroscopic and microscopic level using scanning tunneling microscopy ${ }^{46}$ and electrochemical measurements of surface texture and surface area ${ }^{4}$. Macroscopically, the STM images show that the electrode surface is composed of atomically-terraced crystallites $\sim 25 \mathrm{~nm}$ in diameter. The electrodes have a roughness factor of $\sim 1.3$, which is given as the surface area determined from the oxidative desorption of iodine ${ }^{4}$ divided by the geometric electrode area.

\section{Monolayer preparation}

The carboxylic acid-terminated, and methyl-terminated alkanethiolate monolayers were prepared by their spontaneous adsorption onto the evaporated gold from $0.1 \mathrm{mM}$ solutions in absolute ethanol. For the hydrogen-bonded dimeric $\mathbf{C}=\mathbf{O}$ monolayers, the substrates were placed in solution for $4 \mathrm{~min}$., immersed, rinsed successively with absolute ethanol and hexane, and dried under a stream of prepurified $\mathrm{N}_{2}$. More than $30 \mathrm{~min}$ is needed to form free $\mathrm{C}=\mathrm{O}$ form monolayers. 


\section{Emersion measurements}

Prior to the emersion measurements, all the samples were immersed in $\mathrm{pH} 1.0 \mathrm{HCl}$ aqueous solution for $30 \mathrm{~min}$. in order to obtain more stable structures. The emersion experiments were performed by the slow withdrawal of the monolayer-coated substrates from aqueous solutions of varied $\mathrm{pH}$. Typically, samples were left in solution for $\sim 1 \mathrm{~min}$, with immersions of up to $\sim 1 \mathrm{~h}$ yielding essentially equivalent results. Upon removal from solution, the samples were placed on a spin-coater and spun briefly at $2000 \mathrm{rpm}$ to remove excess solution. The samples were then placed in the $\mathrm{N}_{2}$-purged sample chamber of the IR spectrometer for characterization. Spectra were usually acquired within a few minutes of loading in the chamber, with the rotational vibrational bands of vapor phase water subtracted by using a reference vapor spectrum. All solutions were prepared at an ionic strength of $0.1 \mathrm{M}$ by using $\mathrm{KCl}$. Comparable results were obtained in both buffered and unbuffered solution.

\section{Infrared spectroscopy}

Infrared spectra were acquired with either a Nicolet 740 or a 750 FT-IR spectrometer. Spectra of the adsorbate precursors were obtained by their dispersion in $\mathrm{KBr}$. Spectra of the monolayers were obtained in a single reflection mode by using p-polarized light incident at $82^{\circ}$ from the surface normal and a liquid nitrogen cooled $\mathrm{HgCdTe}$ detector. A home-built sample holder was used to position reproducibly the substrates in the sample chamber of the spectrometer ${ }^{47}$. The spectrometer was purged with boil-off from liquid $\mathrm{N}_{2}$. Spectra were obtained by referencing 1024 sample scans to 1024 background scans at $2 \mathrm{~cm}^{-1}$ resolution (zero filled) with Happ-Genzel apodization. All spectra are reported as $-\log \left(R / R_{0}\right)$, where $R$ is the reflectance of the coated substrate and $R_{0}$ is that of a reference $A u$ substrate. The reference substrates were prepared by immersion of an uncoated gold substrate into $\sim 1 \mathrm{mM}$ ethanolic solution of octadecanethiol- $\mathrm{d}_{37}$ for 24 hours. These films provide a substrate free from detectable $\mathbb{R}$ bands in the regions relevant to these studies as determined when referenced to an uncoated Au substrate that was cleaned in a X 1:3 H $\mathrm{H}_{2} \mathrm{O}_{2}(30 \%): \mathrm{H}_{2} \mathrm{SO}_{4}$ (con.) solution. Further details of the preparation of the reference substrates are given elsewhere ${ }^{48}$. Caution: 
The $\mathrm{H}_{2} \mathrm{O}_{2} / \mathrm{H}_{2} \mathrm{SO}_{4}$ solution reacts violently with organic compounds and should be handled with extreme care.

The orientation analysis, which compares the observed spectrum to that calculated for an isotropic array of oscillators of comparable packing density, was accomplished by using the frequency-dependent complex optical constants of the adsorbate precursor or a suitable substitute. The optical constants for 16-mercaptohexadecanoic acid was determined from a transmission spectrum after its dispersion as a crystalline material in $\mathrm{KBr}$. From the absorbance values, a Kramers-Kronig transformation ${ }^{49}$ yielded the frequency-dependent complex optical constant. The optical constants of octadecanethiol from a comparable data workup were used for tetradecanethiol, which is a liquid at room temperature.

\section{Chemicals and reagents}

$$
\text { Chemicals of } \mathrm{CH}_{3}\left(\mathrm{CH}_{2}\right)_{13} \mathrm{SH} \text { (TDT), } \mathrm{HO}_{2} \mathrm{C}\left(\mathrm{CH}_{2}\right)_{2} \mathrm{SH} \text { (MPA), }
$$

$\mathrm{HCl}$ and $\mathrm{KOH}$ were obtained from Aldrich. The carboxylic acidterminated thiols inculding $\mathrm{HO}_{2} \mathrm{C}\left(\mathrm{CH}_{2}\right)_{10} \mathrm{SH}(\mathrm{MUA})$, and $\mathrm{HO}_{2} \mathrm{C}\left(\mathrm{CH}_{2}\right)_{15} \mathrm{SH}(\mathrm{MHA})$ were synthesized in this group and characterized with $\mathbb{I R}$ and NMR. The disulfide $\left(\mathrm{CH}_{3}\left(\mathrm{CH}_{2}\right)_{4}\right) \mathrm{SS}\left(\left(\mathrm{CH}_{2}\right)_{10} \mathrm{CO}_{2} \mathrm{H}\right)$ (PUAD) was a gift from Dr. Fred Sun (Oregon Graduate Institute of Science \& Technology). The alkanethiols were purified prior to use by passage through an activity one neutral alumina column. All other reagents were used as received. Aqueous solutions were freshly prepared from water purified by passage through a Milli-Q purification water system (Millipore Corp. Bedford, MA). 


\section{RESULTS AND DISCUSSION}

We have demonstrated that the coverage and the orientation of a MHA monolayer on gold are a function of immersion time ${ }^{50}$. After in $0.1 \mathrm{mM}$ solution for only $4 \mathrm{~min}$, the fractional coverage of the MHA/Au monolayer increases from $0 \%$ to $65 \%$ and the average tilt angle of the polymethylene chain of the monolayer decreases from $78^{\circ}$ to $46^{\circ}$. On the other hand, the twist angle remains virtually the same with a value of $\sim 52^{\circ}{ }^{16}$ regardless the coverage. This indicates that although there is a substantial decrease in the average tilt angle, the Au-S-C angle is always almost $120^{\circ} .{ }^{16}$ In addition to the non-hydrogen-bonded and the hydrogen-bonded dimeric $v(\mathrm{C}=\mathrm{O})$ peaks, there is a hydrogen-bonded polymeric $v(C=0)$ bond at $1680 \mathrm{~cm}^{-1}$. When the coverage is low, the hydrogen-bonded dimeric $v(\mathrm{C}=0)$ mode is the only one observed. As the coverage increases, the hydrogen-bonded dimeric $v(C=O)$ peak gradually splits into both the non-hydrogen-bonded $v(\mathrm{C}=\mathrm{O})$ and the hydrogen-bonded polymeric $v(C=0)$ peaks at the same time. The average tilt angle of the $v(C=0)$ dipole increases from $32^{\circ}$ to $64^{\circ}$ in order to form more stable side-to-side network type hydrogen bond. The result of this is a significant increase in the wetting properties of the film ${ }^{50}$.

Peak positions, mode assignments, and transition dipole moment directions (for an all-trans chain) of the MHA/Au monolayer are listed in Table $1^{19,51}$.

The spectra in Figure 1 contain several important compositional and structural details. From a compositional viewpoint, the absence of the $v_{\mathrm{a}}\left(\mathrm{CH}_{3}\right.$,ip $)$ at $2964 \mathrm{~cm}^{-1}$, the $v_{\mathrm{s}}\left(\mathrm{CH}_{3}, \mathrm{FR}_{1}\right)$ at $2936 \mathrm{~cm}^{-1}$, the $v_{\mathrm{S}}\left(\mathrm{CH}_{3}, \mathrm{FR}_{2}\right)$ at $2878 \mathrm{~cm}^{-1}$, and the $\delta\left(\mathrm{CH}_{3}\right)$ at $1382 \mathrm{~cm}^{-1}$ in Figure 1 indirectly confirms the formation of the carboxylic acid-terminated layer, and the presence of the $v_{\mathrm{a}}\left(\mathrm{CH}_{2}\right)$ at $2918 \mathrm{~cm}^{-1}$ with $v_{\mathrm{s}}\left(\mathrm{CH}_{2}\right)$ at $2850 \mathrm{~cm}^{-1}$ indicates the existence of the methylene chain in this monolayer.

The $v_{\mathrm{a}}\left(\mathrm{CH}_{2}\right)$ at $2918 \mathrm{~cm}^{-1}$ and the $v_{\mathrm{s}}\left(\mathrm{CH}_{2}\right)$ at $2850 \mathrm{~cm}^{-1}$ modes remain almost independent of the $\mathrm{pH}$ of immersion solution as shown in 
Table 1. Peak positions, mode assignments, and transition dipole moment directions of carboxylic acid- and methyl-terminated alkanethiolate monolayers at gold exposed prior to emersion experiments.

\section{$\mathrm{HO}_{2} \mathrm{C}\left(\mathrm{CH}_{2}\right)_{15} \mathrm{~S} / \mathrm{Au} \quad \mathrm{CH}_{3}\left(\mathrm{CH}_{2}\right)_{13} \mathrm{~S} / \mathrm{Au}$}

\begin{tabular}{|c|c|c|c|c|c|}
\hline $\begin{array}{l}\text { Mode assignment } \\
\text { dipole }^{\mathrm{a}}\end{array}$ & obs. & calc. & obs. & calc. & $\begin{array}{l}\text { direction of } \\
\text { transition }\end{array}$ \\
\hline$v_{\mathrm{a}}\left(\mathrm{CH}_{3, \mathrm{ip}}\right)$ & & & 2964 & & $\begin{array}{l}\perp \text { to } \mathrm{C}-\mathrm{CH}_{3} \text { bond in } \\
\mathrm{C}-\mathrm{C}-\mathrm{C} \text { chain plane }\end{array}$ \\
\hline$v_{\mathrm{a}}\left(\mathrm{CH}_{3, \mathrm{op}}\right)$ & & & b & 2954 & $\begin{array}{l}\perp \text { to } \mathrm{C}-\mathrm{CH}_{3} \text { bond } \perp \\
\mathrm{C}-\mathrm{C}-\mathrm{C} \text { chain plane }\end{array}$ \\
\hline$v_{\mathrm{s}}\left(\mathrm{CH}_{3}, \mathrm{FR}_{1}\right)^{\mathrm{c}}$ & & & 2936 & & $\|$ to $\mathrm{C}-\mathrm{CH}_{3}$ bond \\
\hline$v_{\mathrm{a}}\left(\mathrm{CH}_{2}\right)$ & 2918 & 2920 & 2918 & 2920 & $\begin{array}{l}\perp \text { to } C-C-C \text { chain } \\
\text { plane }\end{array}$ \\
\hline$v_{\mathrm{S}}\left(\mathrm{CH}_{3}, \mathrm{FR}_{2}\right)^{\mathrm{c}}$ & & & 2878 & & $\|$ to $\mathrm{C}-\mathrm{CH}_{3}$ bond \\
\hline$v_{\mathrm{s}}\left(\mathrm{CH}_{2}\right)$ & 2850 & 2850 & 2850 & 2851 & $\begin{array}{l}\text { \|l to C-C-C plane } \\
\text { bisceting } \mathrm{H}-\mathrm{C}-\mathrm{H}\end{array}$ \\
\hline$v(C=0)$ & $\begin{array}{c}1743 \\
1717,1680\end{array}$ & 1699 & & & $\|$ to $\mathrm{C}=\mathrm{O}$ bond \\
\hline$\delta\left(\mathrm{CH}_{2}\right)$ & 1460 & 1472 & 1468 & 1467 & $\begin{array}{l}\text { in } \mathrm{HCH} \text { plane, } \\
\text { bisecting } \mathrm{HCH}\end{array}$ \\
\hline$v(\mathrm{C}-\mathrm{O})+\delta(\mathrm{C}-\mathrm{O}-\mathrm{H})^{\mathrm{d}}$ & 143 & & & & $\|$ to $\mathrm{C}-\mathrm{O}$ bond \\
\hline$\delta_{\mathrm{s}}\left(\mathrm{CH}_{3}\right)$ & 1382 & 1382 & 1380 & $1375^{e}$ & $\|$ to $\mathrm{C}-\mathrm{CH}_{3}$ bond \\
\hline$\omega\left(\mathrm{CH}_{2}\right)$ & 1382 & 1382 & 1380 & 1380 & \|t to axis of chain \\
\hline and twists & 1170 & & 1344 & 1170 & \\
\hline
\end{tabular}


Table 1. (continued)

aOrientation of transition dipoles for $\mathrm{CH}$ modes assuming all-trans conformation (Snyder, R. G. J. Chem. Phys. 1965, 42, 1744). bThe $v_{\mathrm{a}}\left(\mathrm{CH}_{3, \mathrm{op}}\right)$ mode was not observed in the monolayer spectrum. ' $T$ The fermi-resonance couplet, $v_{\mathrm{s}}\left(\mathrm{CH}_{3}, \mathrm{FR}_{1}\right)$ and $v_{\mathrm{s}}\left(\mathrm{CH}_{3}, \mathrm{FR}_{2}\right)$, is designated by the subscripts 1 and 2 , which refer to the higher and lower energy components, respectively. $d$ This band involves both the stretching of the $\mathrm{C}-\mathrm{O}$ bond and the deformation of the $\mathrm{C}-\mathrm{O}-\mathrm{H}$ angle (D. Hadzi and $\mathrm{M}$.

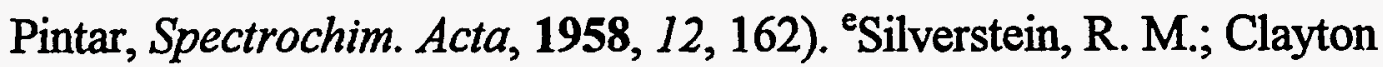
Bassler, G.; Morrill, T. C. Spectrometric Identification of Organic Compuonds 5th ed.; John Wiley \& Sons, Inc.: New York, 1991. 


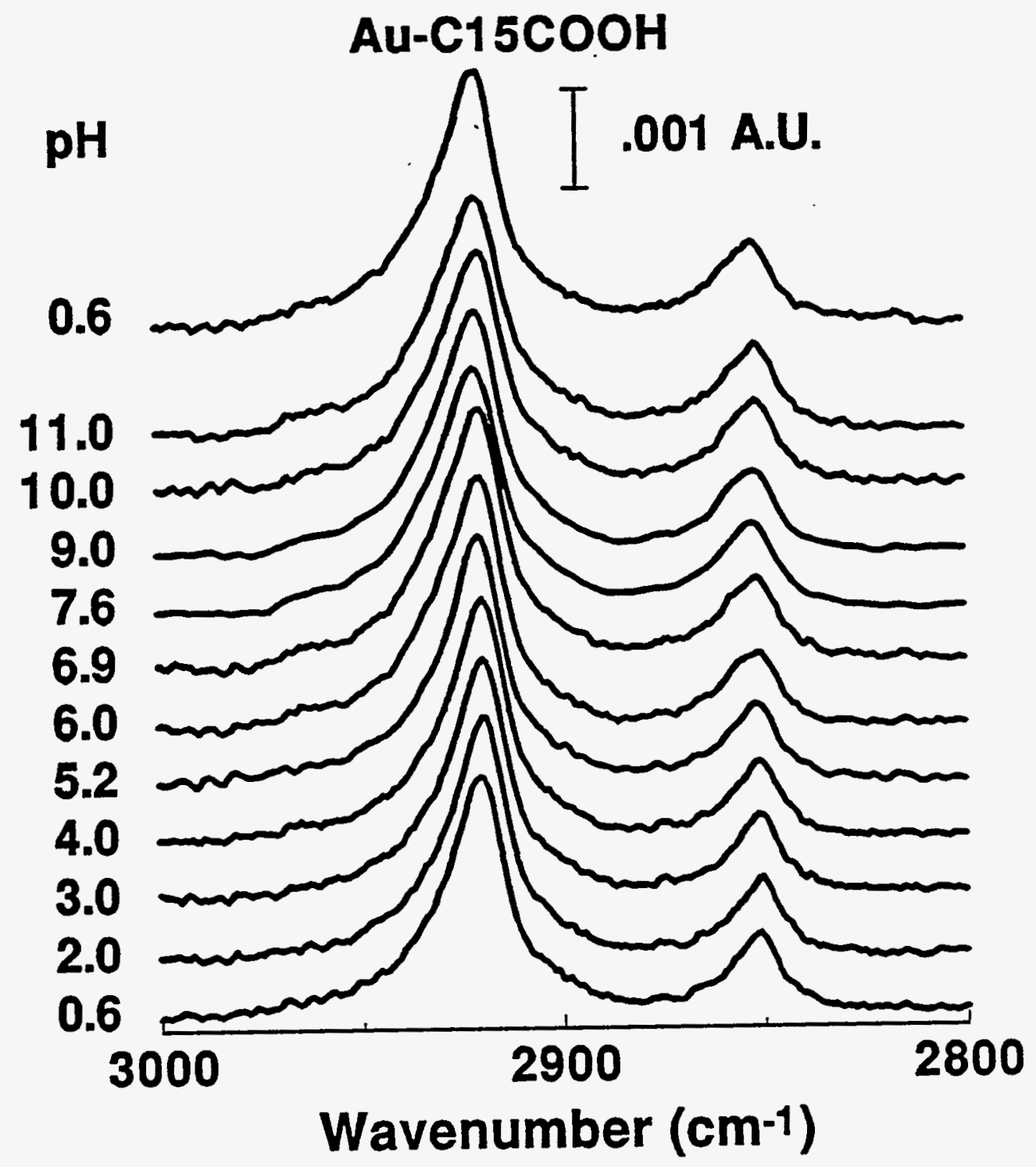

Figure 1. IRRAS spectra in the high energy region for a $\mathrm{HO}_{2} \mathrm{C}\left(\mathrm{CH}_{2}\right)_{15} \mathrm{~S} / \mathrm{Au}$ monolayer with a fraction coverage of 0.65 as a function of solution $\mathrm{pH}$. 
Figure 1. This implies that the orientation of the polymethylene chain is always the same regardless of the solution $\mathrm{pH}$. That is, there is no solvent or electrolyte incorporated in the film due to the deprotonation of the carboxylic group. This further confirms that the monolayer has a denselypacked structure. This conservation of the orientation of the polymethylene chain is the fundamentally important corner stone for the IRRAS studies of the $\mathrm{C}=\mathrm{O}$ modes since the absorption is a function of the orientation as well.

In Figure 2, the presence of the $v(C=0)$ envelope between 1650 and $1760 \mathrm{~cm}^{-1}$, coupled with the absence of the aforementioned methyl bands, verify the construction of the acid-terminated layer.

Figure 3 shows the expanded IRRAS spectra in the $v(C=0)$ region as a function of solution $\mathrm{pH}$.

\section{Methyl-terminated monolavers}

Spectra for the methyl-terminated layer are given in Figures $\mathbf{4}$ and 5 for the sequential emersion from $\mathrm{pH} 1.0,6.0$, and 12.7 solutions. Spectra are also given for the emersion from the $\mathrm{pH} 1.0$ solution after emersion from the $\mathrm{pH} 12.7$ solution. The spectra in all instances are essentially identical to that measured initially at $\mathrm{pH} 1.0$. This is further supported by the different spectra given in the insert of Figures 4 and 5 , which were calculated by dividing the spectrum at a given $\mathrm{pH}$ by that of the initial pH 1.0 emersion. These results indicate that the methylterminated layer is unaffected by immersion into aqueous solutions of varied $\mathrm{pH}$. Immersion times up to $\sim 1$ hour did not alter the observations and agree with extensive stability studies reported earlier ${ }^{1,14}$.

\section{MHA monolayers}

MHA monolayers with a fractional coverage of 0.65

The IRRAS spectra for the emersion into solutions of varied $\mathrm{pH}$ of the MHA/Au monolayer which has the hydrogen-bonded dimeric $\mathrm{C}=\mathrm{O}$ form as the majority are shown in the low and high energy regions in 


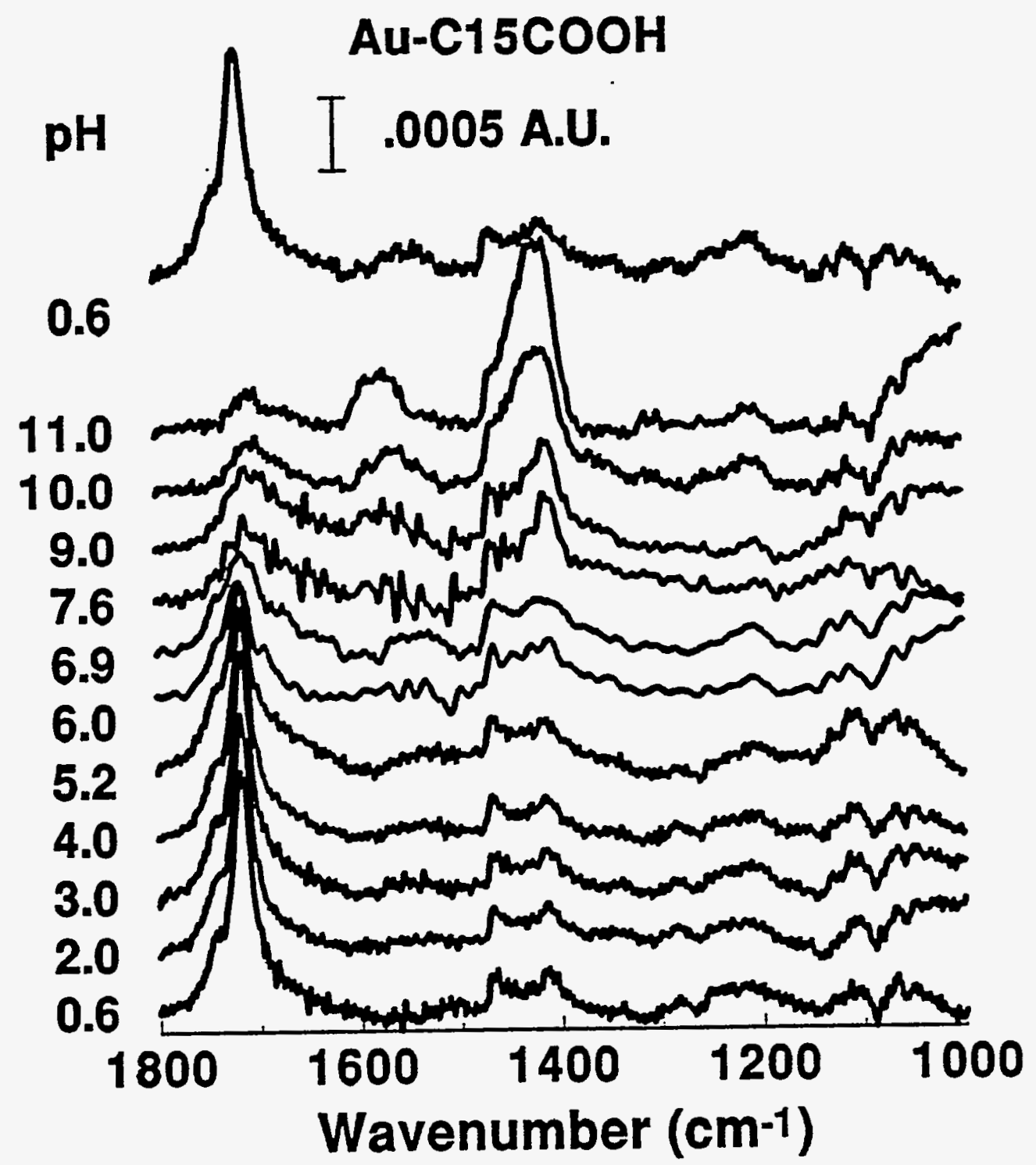

Figure 2. IRRAS spectra in the low energy region for a $\mathrm{HO}_{2} \mathrm{C}\left(\mathrm{CH}_{2}\right)_{15} \mathrm{~S} / \mathrm{Au}$ monolayer with a fraction coverage of 0.65 as a function of solution $\mathrm{pH}$. 


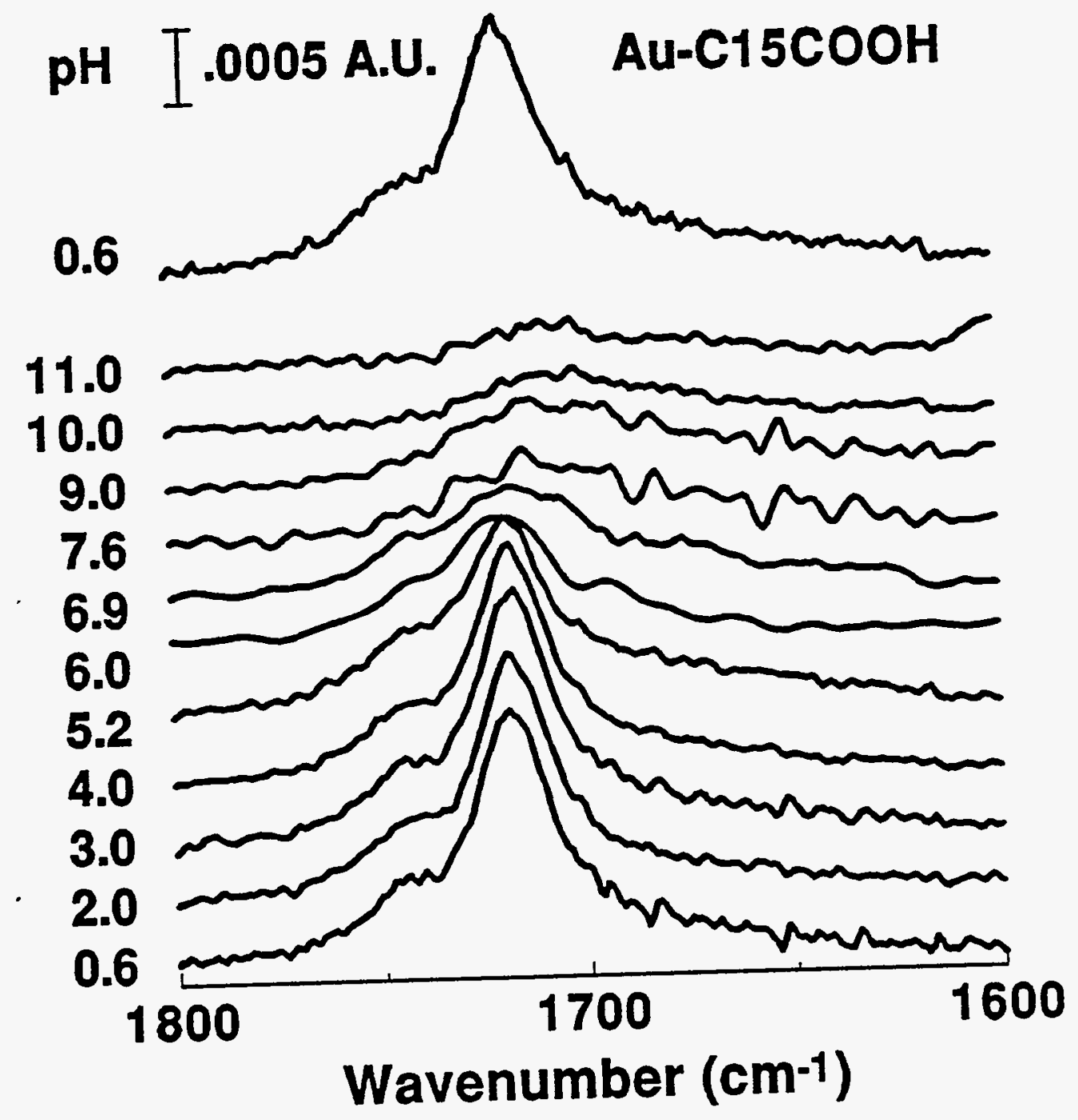

Figure 3. IRRAS spectra in $v(\mathrm{C}=\mathrm{O})$ region for a $\mathrm{HO}_{2} \mathrm{C}\left(\mathrm{CH}_{2}\right)_{15} \mathrm{~S} / \mathrm{Au}$ monolayer with a fraction coverage of 0.65 as a function of solution $\mathrm{pH}$. 


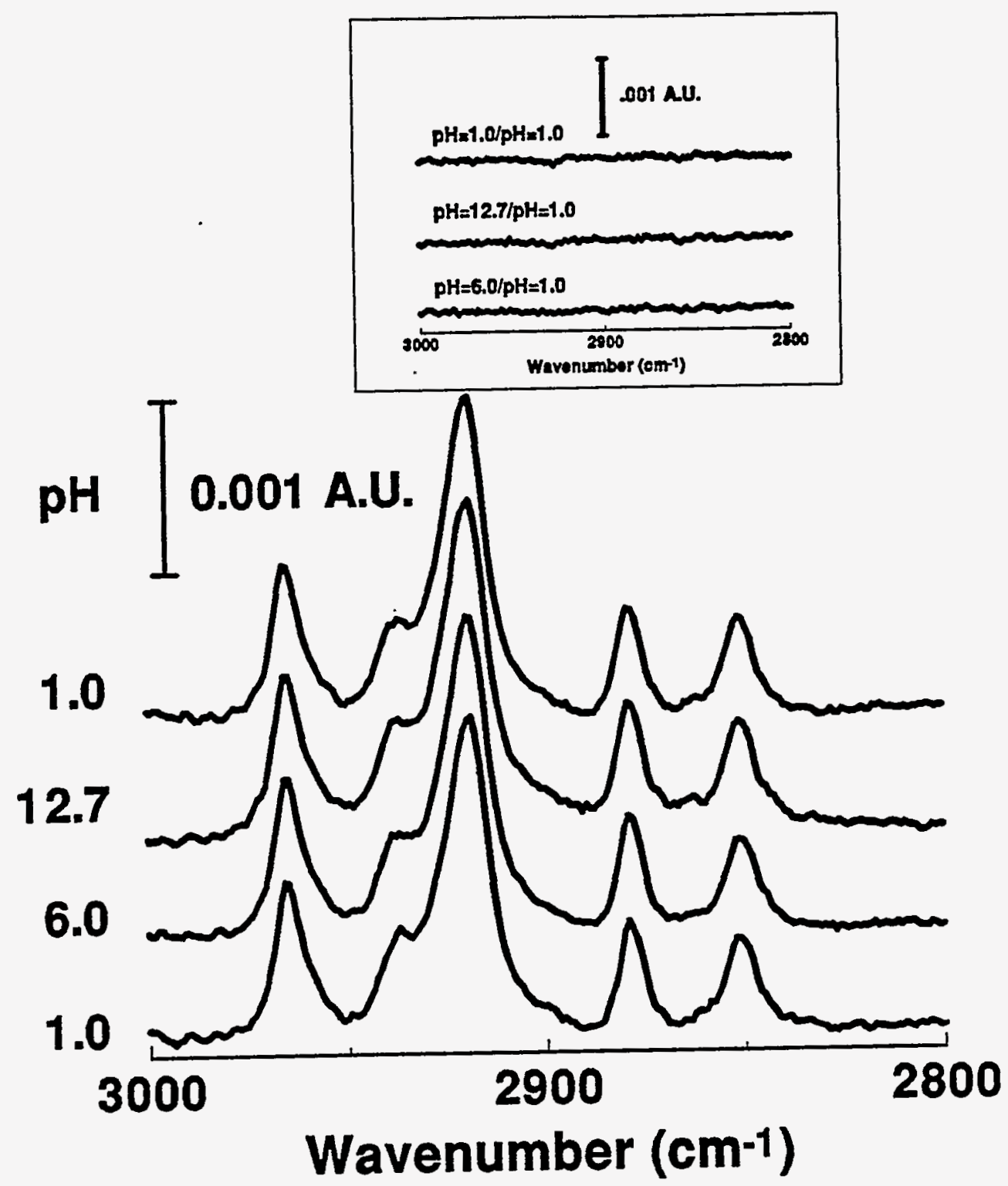

Figure 4. IRRAS spectra in the high energy region for a monolayer of $\mathrm{CH}_{3}\left(\mathrm{CH}_{2}\right)_{13} \mathrm{~S}$ at gold as a function of solution $\mathrm{pH}$. Inset: difference spectra using the spectrum at $\mathrm{pH} 1.0$ as the reference spectrum. 


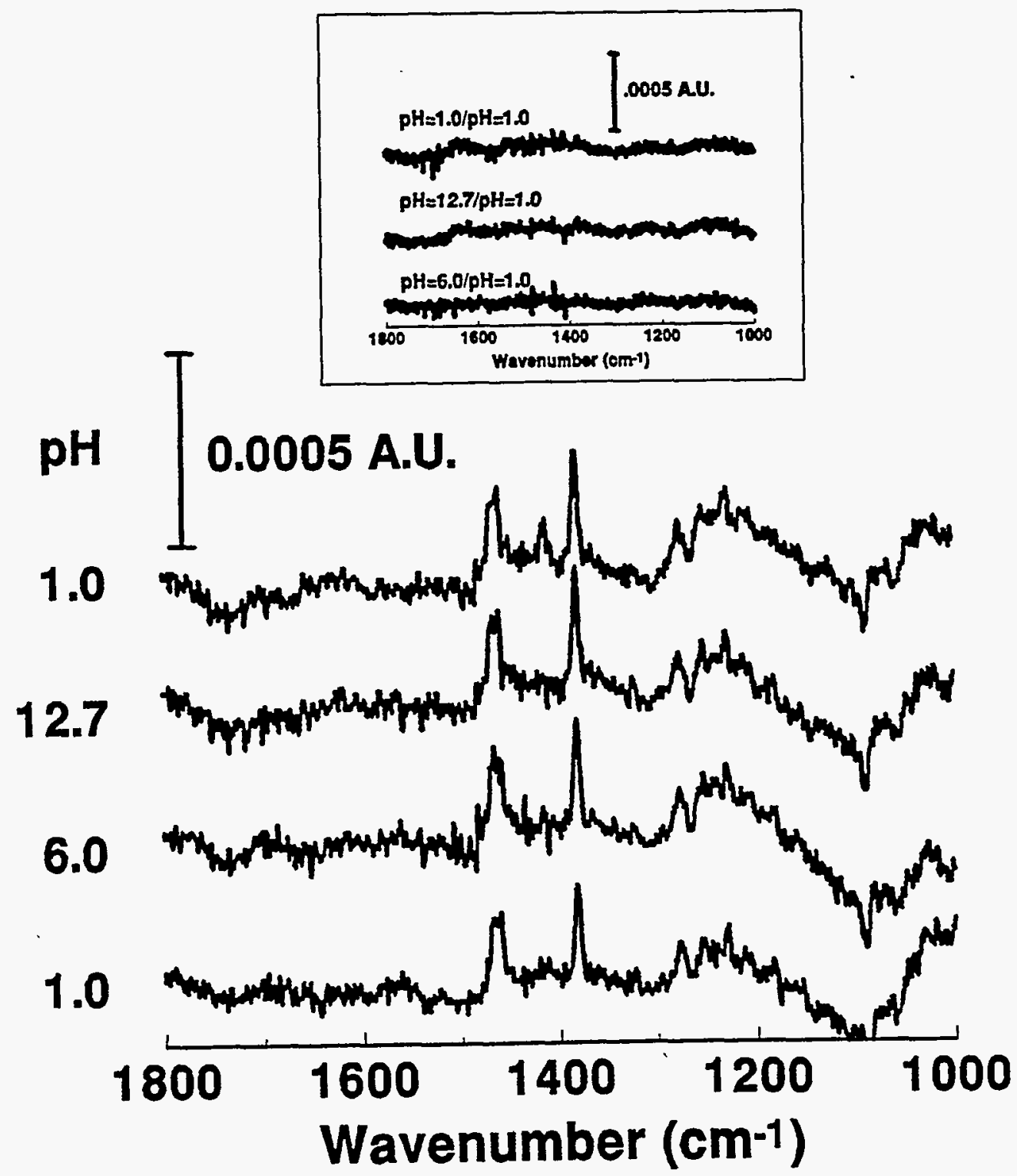

Figure 5. IRRAS spectra in the low energy region for a monolayer of $\mathrm{CH}_{3}\left(\mathrm{CH}_{2}\right)_{13} \mathrm{~S}$ at gold as a function of solution $\mathrm{pH}$. Inset: difference spectra using the spectrum at $\mathrm{pH} 1.0$ as the reference spectrum. 
Figures 2 and 1, respectively. The $\mathrm{pH}$ of the immersion solutions was increased from an initial value of 0.6 to a final value of 11.0. A spectrum is also given for the re-immersion of the sample into the $\mathrm{pH} 0.6$ solution after cycling through the noted sequence. Spectra above a $\mathrm{pH}$ of 11.0 for these layers were masked by intense, poorly-defined features, which we attributed to the relatively large amount of residual electrolyte as shown in Figure 6. Changes in the spectra are most evident in the carbonyl stretching region at a $\mathrm{pH}$ of 6.0 and greater. Little change is observable in the spectra below this value. Following the earlier band assignments, the changes in the spectra are consistent with the progressive ionization of the acidic end groups as the $\mathrm{pH}$ of the immersion solution increases.

The changes in the carbonyl stretching region are, however, not accompanied at an observable level by the expected changes in the spectral regions attributed to the symmetric $v_{\mathrm{a}}(\mathrm{COO}-)$ or asymmetric $v_{\mathrm{s}}(\mathrm{COO}-)$ carboxylate stretching modes until above a $\mathrm{pH}$ of 9.0 . The former is generally observed between $1600 \mathrm{~cm}^{-1}$ and $1550 \mathrm{~cm}^{-1}$, and the latter between $1450 \mathrm{~cm}^{-1}$ and $1400 \mathrm{~cm}^{-1}$. $52-55$ Though the overlap with $\delta\left(\mathrm{CH}_{2}\right), \delta\left(\mathrm{a}-\mathrm{CH}_{2}\right)$, and $\gamma(\mathrm{C}-\mathrm{O})+\delta(\mathrm{O}-\mathrm{H})$ modes complicates an interpretation of the $v_{\mathrm{S}}(\mathrm{COO}-)$ region, no observable change is apparent in the $v_{\mathrm{a}}(\mathrm{COO}-)$ region. Based on the assumed orientation of the end group, this observation is unexpected, suggesting the possible structural rearrangement of the chain terminus upon ionization. Such changes would be driven by charge repulsion and/or counter-ion association effects. Experiments with diluted layers and with immersion solutions of varied ionic strength and composition are planned to examine this issue further. The MHA monolayer with not only the dimeric and the polymeric forms but also a free form will be discussed. Although the peak intensity of the dimeric $\mathrm{C}=\mathrm{O}$ form is smaller than that of the monolayer without free form, it behaves similarly to the latter.

To gain further insight into the reactivity of these layers, the envelope in the $v(C=0)$ region was deconvoluted into its three major 


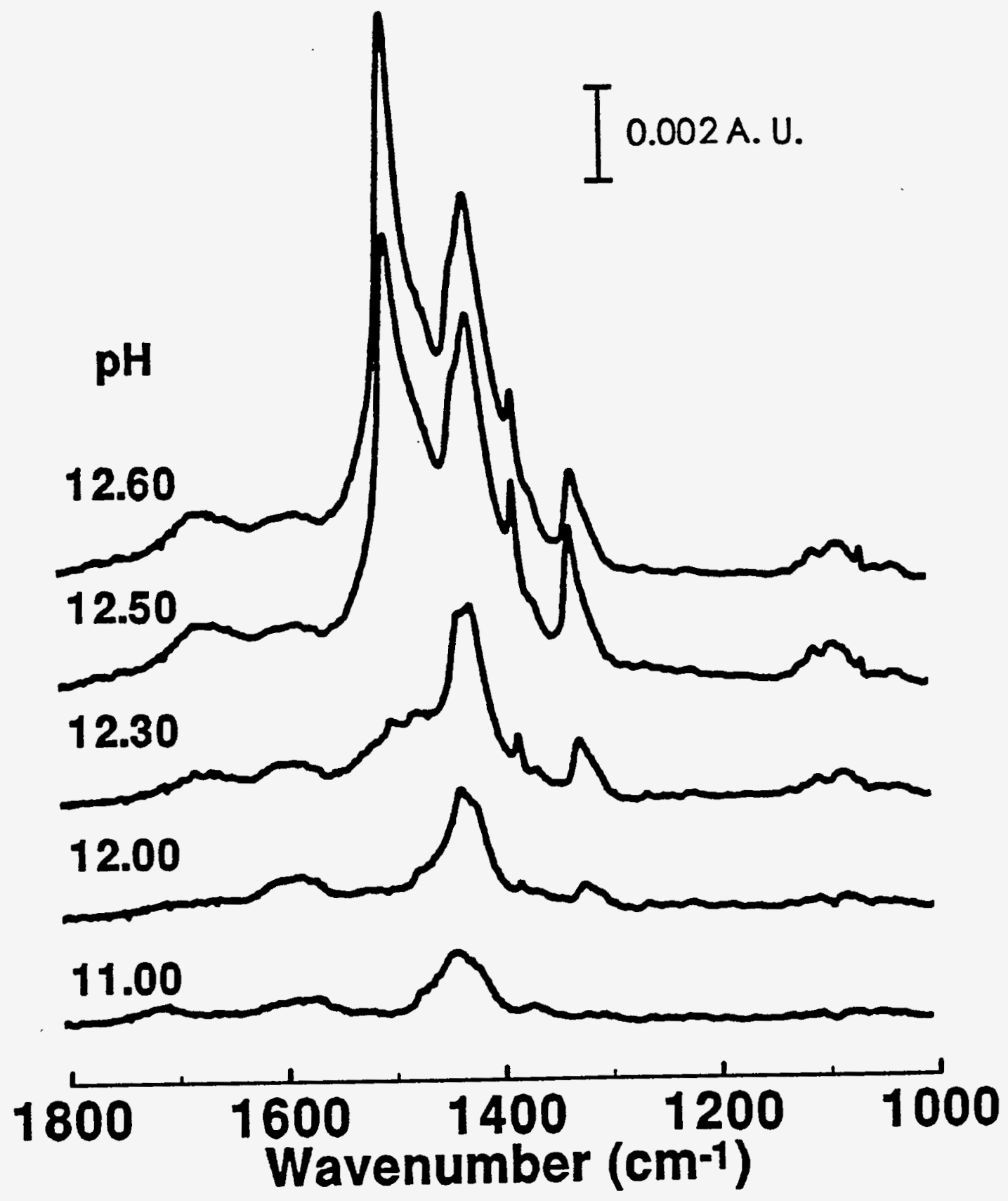

Figure 6. IRRAS spectra in the low energy region for multilayers of $\mathrm{KOH}$ on top of $\mathrm{HO}_{2} \mathrm{C}\left(\mathrm{CH}_{2}\right)_{15} \mathrm{~S} / \mathrm{Au}$ monolayer as a function of solution $\mathrm{pH}$. 
component peaks: the free acid, the hydrogen-bonded dimeric acid, and the hydrogen-bonded polymeric acid. The results of the deconvolution for the initial $\mathrm{pH} 0.6$ emersion sample is shown in Figure 7. Fits to the data assuming three peaks could be varied to a small degree, but the trends in the $\mathrm{pH}$ dependence were consistent. Assuming that the oscillator strengths and orientation of both forms of the acids are comparable yields a layer that is $\sim 10 \%$ of the free acid, $\sim 10 \%$ of the hydrogen-bonded polymeric acid and $\sim 80 \%$ of the hydrogen-bonded dimeric acid. For a monolayer in the same solution for 24 hours, there are $\sim 20 \%$ of free, $\sim 20 \%$ of hydrogen-bonded polymeric acid and $\sim 60 \%$ of the hydrogenbonded dimeric acid. We also note the large width of the hydrogenbonded polymeric acid suggests the possibility of a distribution of chainlengths of the linear network bonding. The results of the deconvolution are summarized as a function of $\mathrm{pH}$ in Figures 8-10. The peak frequency of each peak remains the same with the peak area of each peak decreases quite differently with increasing the solution $\mathrm{pH}$. Figure 11 provides a comparison of the peak area of the free acid, the hydrogen-bonded dimeric acid and the hydrogen-bonded polymeric acid as a function of the solution $\mathrm{pH}$. Assuming the orientation of the each $\mathrm{C}=\mathrm{O}$ dipole does not change, the $\mathrm{pK}_{\mathrm{a}}$ of each acid can be calculated and is equal to the $\mathrm{pH}$ where the decrease in the peak area of each peak is $50 \%$. The calculated $\mathrm{pK}_{\mathrm{a}}$ values for the free acid, the dimeric acid, and the polymeric acid are $6.6,7.0$ and 11.0, respectively, as listed in Table 2. The transition range of the free acid, the dimeric acid, and the polymeric acid are 3,7 and $3 \mathrm{pH}$ units, respectively. Interestingly, the peak area of the free acid is virtually lost by $\mathrm{pH} 8.0$ while that of polymeric acid remains almost intact up to $\mathrm{pH}$ 9.0.

Superimposed is an experimental fit to the data that assumes the reactivity of a simple monobasic acid in a solution of high ionic strength. This data suggest two interesting points. First, the acid has a $\mathrm{pK}_{\mathrm{a}}$ value of 7.1 which is far greater than that, 5.0 , in solution. Second, the transition occurs over an interval of $\sim 6.0 \mathrm{pH}$ units. Together, these results point to 


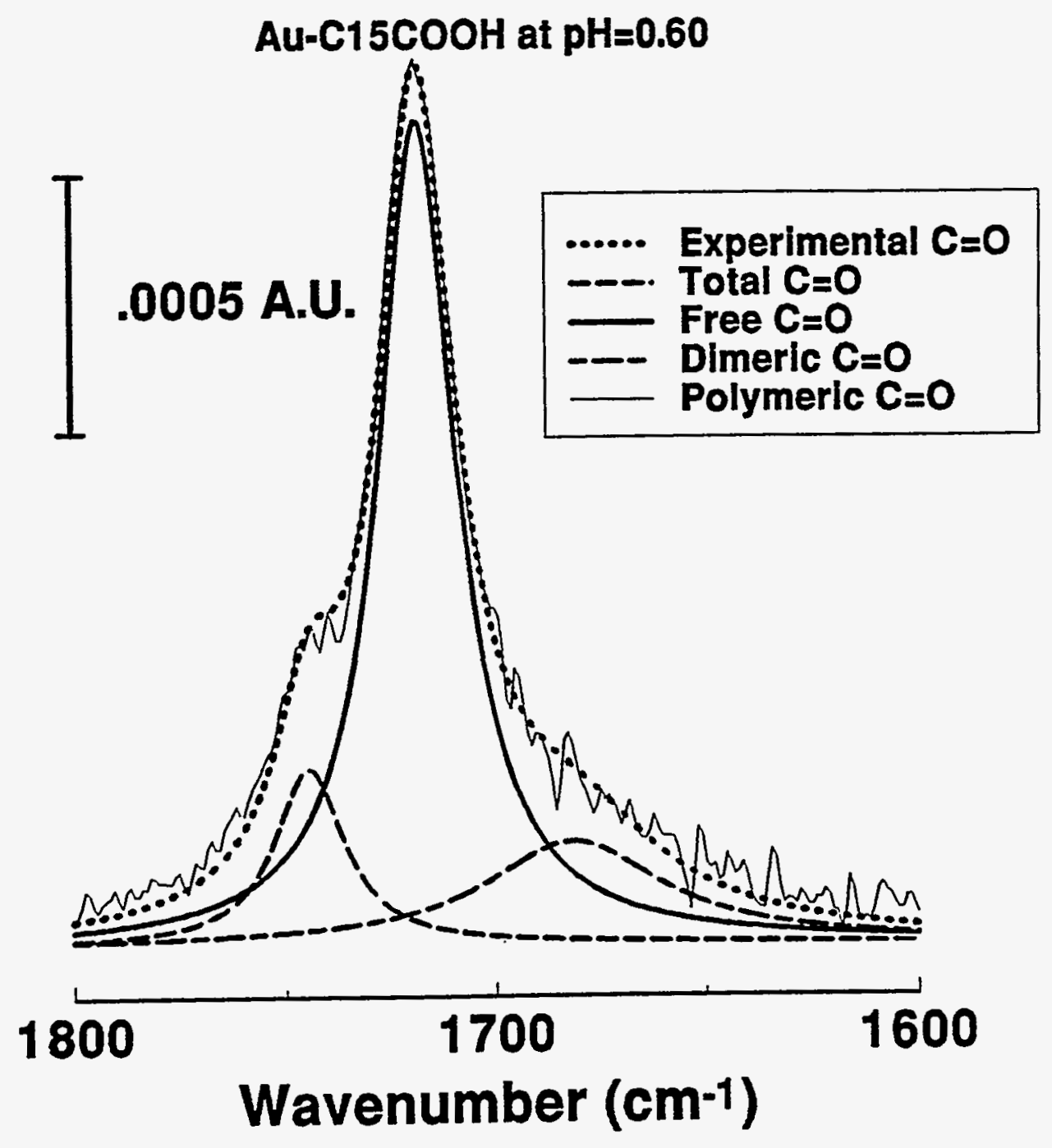

Figure 7. Deconvoluted IRRAS spectra in the $v(C=0)$ region of a $\mathrm{HO}_{2} \mathrm{C}\left(\mathrm{CH}_{2}\right)_{15} \mathrm{~S} / \mathrm{Au}$ monolayer with a fraction coverage of 0.65 emersed in $\mathrm{pH} 0.60 \mathrm{HCl}$ aqueous solution. 

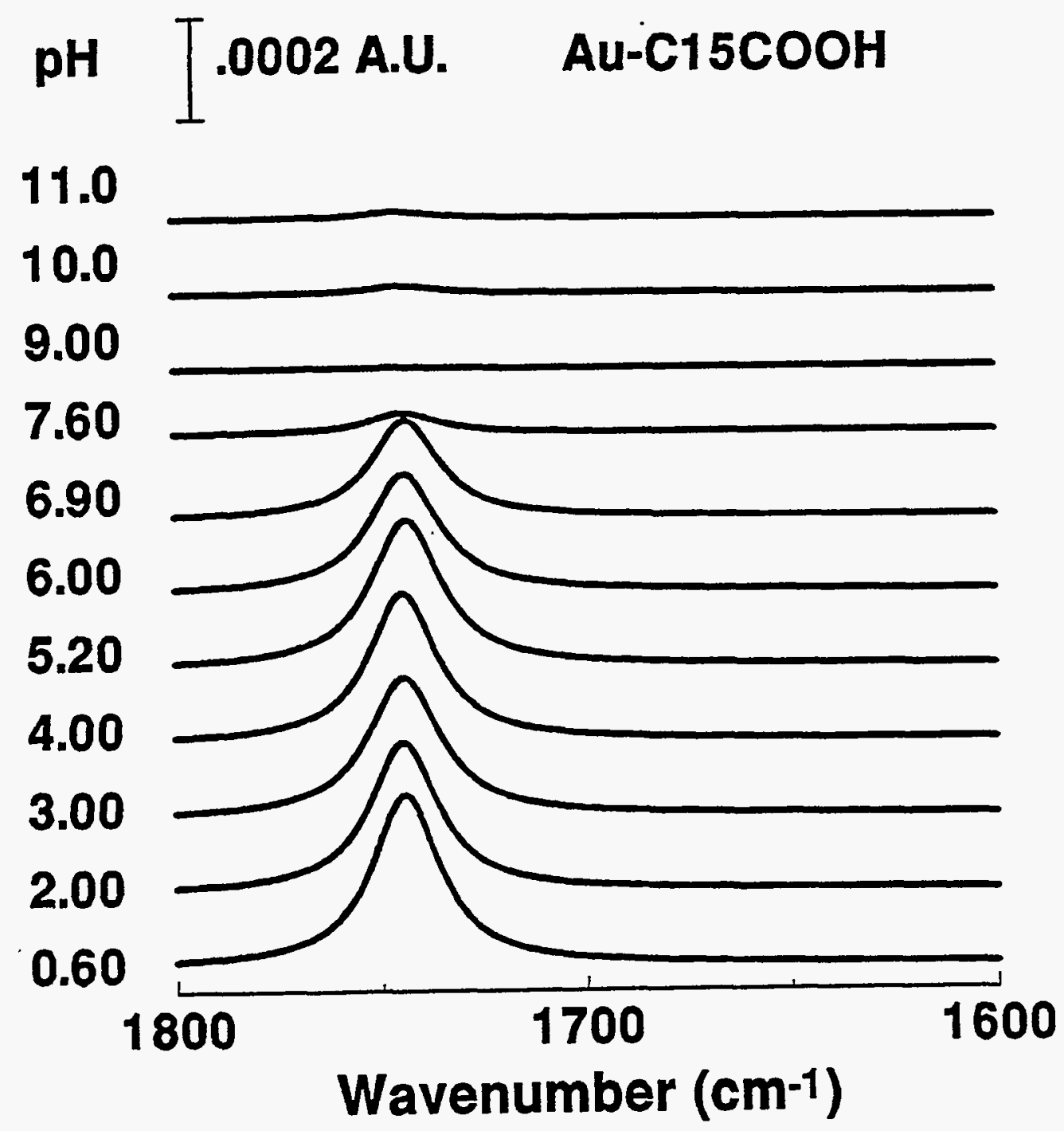

Figure 8. Deconvoluted IRRAS spectra of the free $v(\mathrm{C}=0)$ of a $\mathrm{HO}_{2} \mathrm{C}\left(\mathrm{CH}_{2}\right)_{15} \mathrm{~S} / \mathrm{Au}$ monolayer with a fraction coverage of 0.65 as a function of solution $\mathrm{pH}$. 


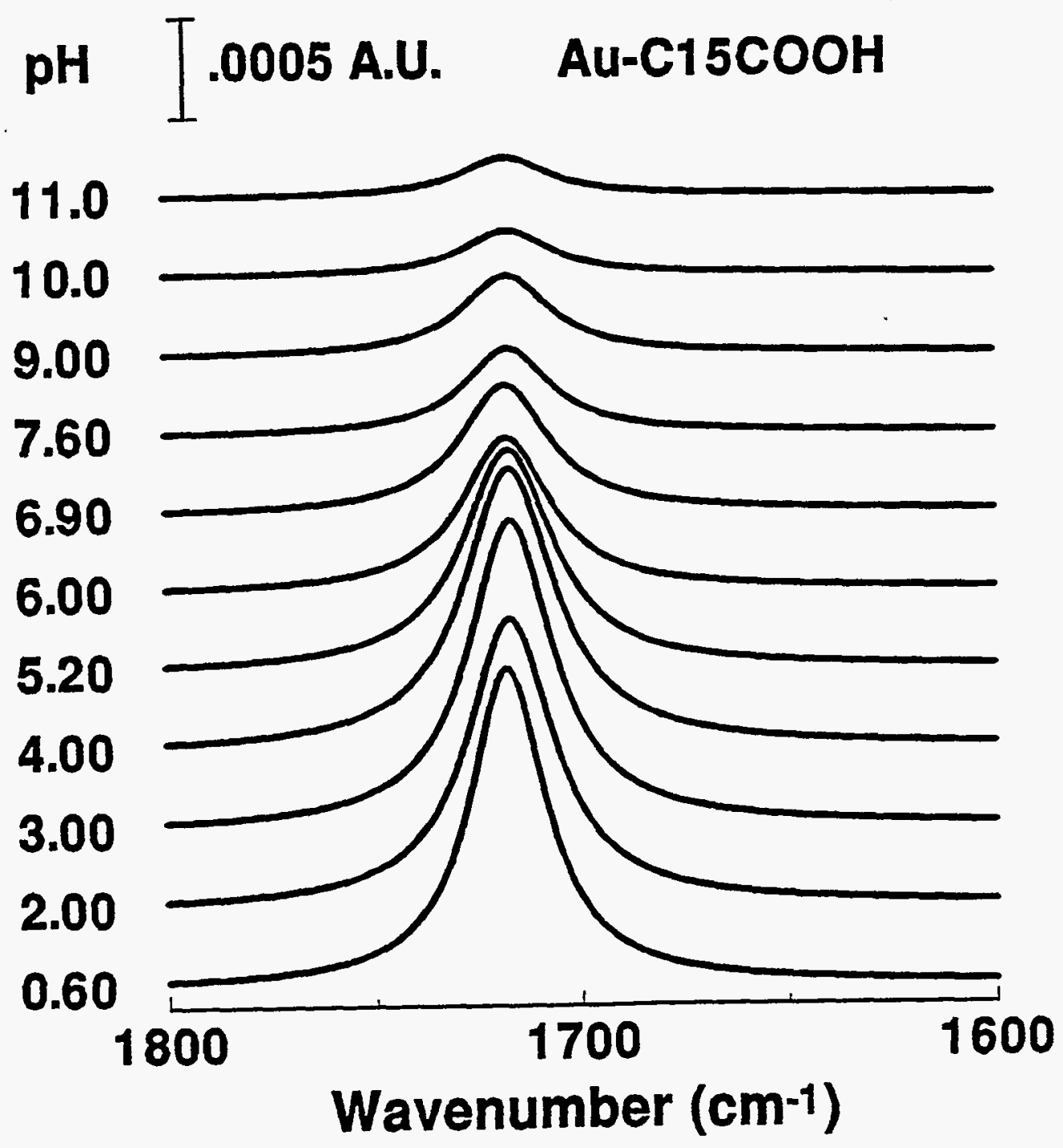

Figure 9. Deconvoluted IRRAS spectra of the hydrogen-bonded dimeric $v(\mathrm{C}=\mathrm{O})$ of a $\mathrm{HO}_{2} \mathrm{C}\left(\mathrm{CH}_{2}\right)_{15} \mathrm{~S} / \mathrm{Au}$ monolayer with a fraction coverage of 0.65 as a function of solution $\mathrm{pH}$. 


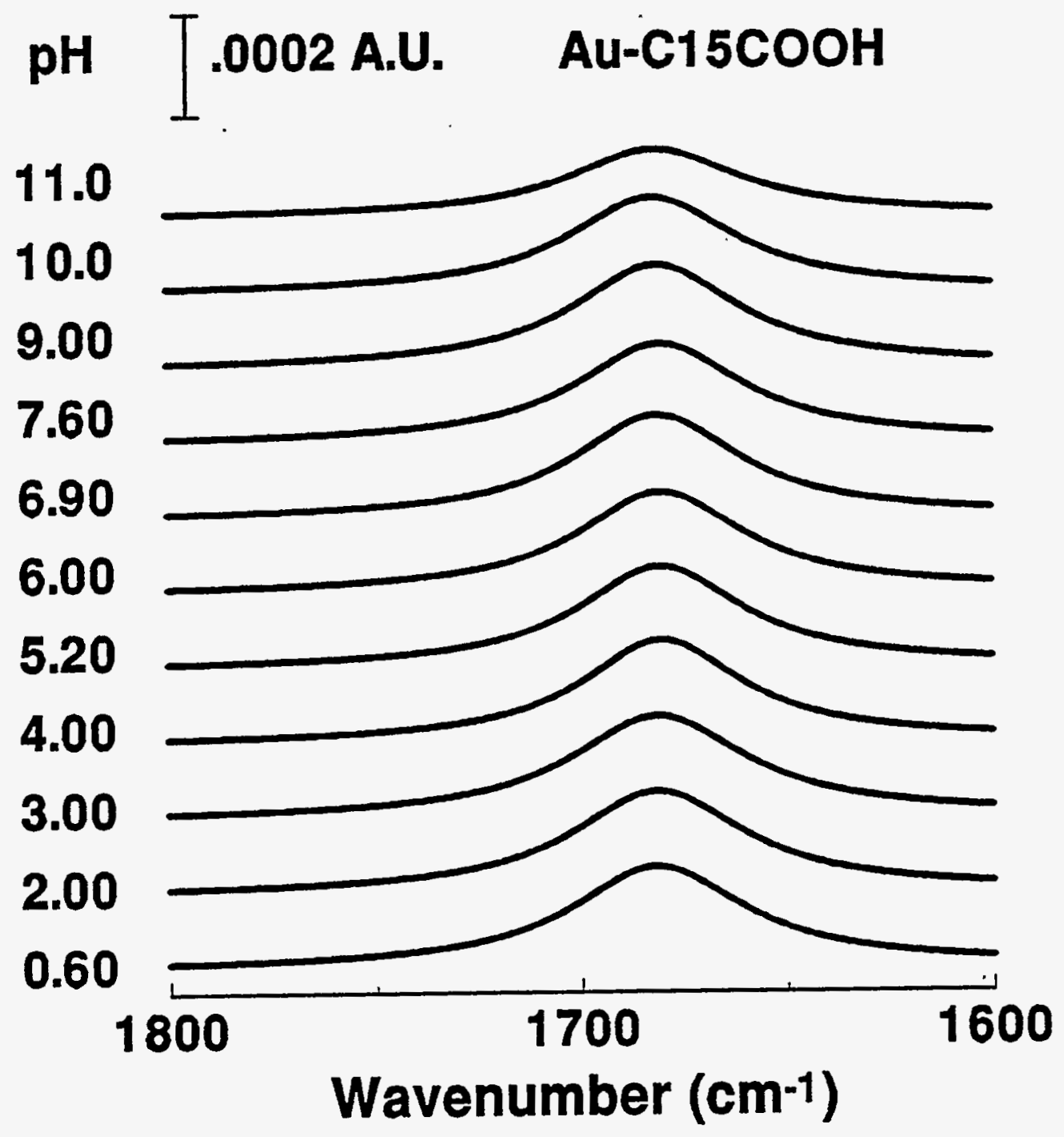

Figure 10. Deconvoluted IRRAS spectra of the hydrogen-bonded polymeric $v(\mathrm{C}=\mathrm{O})$ of a $\mathrm{HO}_{2} \mathrm{C}\left(\mathrm{CH}_{2}\right)_{15} \mathrm{~S} / \mathrm{Au}$ monolayer with a fraction coverage of 0.65 as a function of solution $\mathrm{pH}$. 


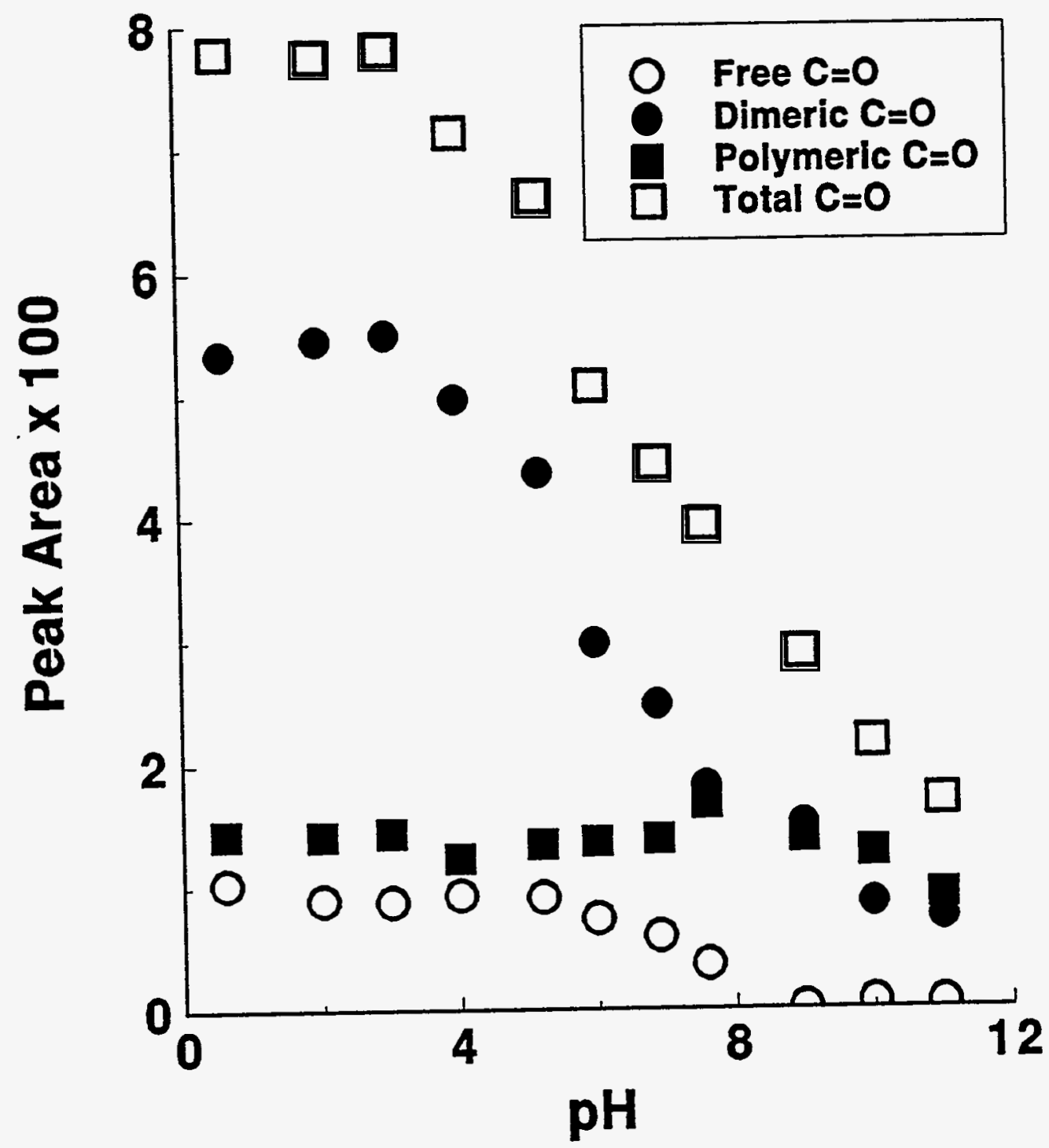

Figure 11. The IRRAS peak area of the $v(\mathrm{C}=0)$ peaks of a $\mathrm{HO}_{2} \mathrm{C}\left(\mathrm{CH}_{2}\right)_{15} \mathrm{~S} / \mathrm{Au}$ monolayer with a fraction coverage of 0.65 as a function of solution $\mathrm{pH}$. 
the free acid acting as a simple monobasic acid which has a strength roughly 10-2 times less than carboxylic acids in solution (consistent with noninteracting site considerations).

The data for the hydrogen-bonded acid is more interesting. It exhibits a continual decrease up to the limiting $\mathrm{pH}$. This behavior is similar to dimers in solution like malonic acid. Actually, there are two different kinds of hydrogen-bonded acids: a dimeric acid and a polymeric acid. The dimeric acid has higher $\mathrm{pH}$ value than that in solution, so it is a weaker acid when it is hydrogen bonded. To the other extremely, polymeric acid has the highest $\mathrm{pK}_{\mathrm{a}}$ of all, because the hydrogen bonding is not just between two molecules but a network of many acid molecules. This strong hydrogen bond makes any acid very difficult to be ionized to give away $\mathrm{H}^{+}$.

The $\mathrm{pK}_{\mathrm{a}}$ and transition rang of the whole monolayer are 7.1 and 9 $\mathrm{pH}$ units. In other words, the acid-terminated monolayers have not only weaker acidity but broader transition $\mathrm{pH}$ units than this acid in aqueous solution.

Effective $\mathrm{pKa}$

To confirm these points, we calculate the degree of ionization, $\alpha_{i}$, of acid and plotted it as a function of the solution $\mathrm{pH}$ as shown in Figure 12. There is only little ionization when solution $\mathrm{pH}$ is less than 4 for all carboxylic acids. The extent of ionization for the free acid starts to rise when the solution $\mathrm{pH}$ is greater than 4 and reaches a value of 1 when the solution $\mathrm{pH}$ is greater than 8 . This trend is very similar to that in aqueous solution. That for the dimeric acid starts to increase after $\mathrm{pH} 4$ and never reaches a value of 1 even at $\mathrm{pH} 12$. That is, there is a transition of at least $8 \mathrm{pH}$ units. This is very possible since these densely-packed molecules are so close together, the electrostatic repulsion among the deprotonated molecules becomes stronger and stronger. As deprotonation continues, there is a greater charge build up in this very small confined space. On the other hand, the value of $\alpha_{i}$ of polymeric acid is virtually 0 until pH 9 . 
Table 2. The $\mathrm{pK}_{\mathrm{a}}$ calculated from IRRAS data for the MHA/Au with a fraction coverage of $0.65(\mathrm{MHA} / \mathrm{Au} / 0.65), \mathrm{MHA} / \mathrm{Au}, \mathrm{MUA} / \mathrm{Au}$, $\mathrm{MPA} / \mathrm{Au}$, and $\mathrm{PUAD} / \mathrm{Au}$.

\begin{tabular}{lcccc}
\multicolumn{1}{c}{$\mathrm{pK}_{\mathrm{a}}$} & free acid & dimeric acid & polymeric acid & overall $\mathrm{pK}_{\mathrm{a}}$ \\
\hline $\mathrm{MHA} / \mathrm{Au} / 0.65$ & 6.6 & 7.0 & 11.0 & 7.1 \\
$\mathrm{MHA} / \mathrm{Au}$ & 6.5 & 7.0 & 11.0 & 7.2 \\
$\mathrm{MUA} / \mathrm{Au}$ & 6.1 & 7.8 & 10.5 & 8.0 \\
$\mathrm{MPA} / \mathrm{Au}$ & 5.3 & 5.7 & 9.5 & 6.0 \\
$\mathrm{PUAD} / \mathrm{Au}$ & $\mathrm{a}$ & $6.5^{\mathrm{a}}$ & $\mathrm{a}$ & $\mathrm{a}$ \\
\hline
\end{tabular}

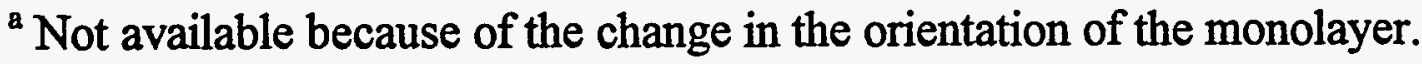


because of the strong hydrogen-bonding.

A plot of the effective $\mathrm{pK}_{\mathrm{a}}$ value for each acid against the degree of ionization, $\alpha_{i}$, of acid will be able to give us a better idea on how theses three different acids behave. The effective $\mathrm{pK}_{\mathrm{\alpha}}$ is calculated using the following equation:

$$
\mathrm{pK}_{\mathrm{a}}^{\mathrm{eff}}=\mathrm{pH}-\log \left(\alpha_{\mathrm{i}} /\left(1-\alpha_{\mathrm{i}}\right)\right)
$$

where $\alpha_{\mathrm{i}}$ is the extent of ionization. This equation relates the extent of ionization at a particular value of $\mathrm{pH}$ to the "effective $\mathrm{pK}_{\mathrm{a}}$ " of monolayers. This "effective $\mathrm{pK}_{\mathrm{a}}$ " is the one that would be attributable to the extent of ionization for a simple monobasic acid. In fact, the $\mathrm{pK}_{\mathrm{a}}$ is independent of $\alpha_{\mathrm{i}}$ for a monobasic acid. For carboxylic acids, the value of $\mathrm{pK}_{\mathrm{a}}$ is reported from 3 to $11^{56-66}$. The effective $\mathrm{pK}_{2}$ of these $\mathrm{C}=\mathrm{O}$ as a function of the extent of ionization are shown in Figure 13. Since both the free acid and the hydrogen-bonded dimeric acid have the effective $\mathrm{pK}_{\mathrm{a}}$ of $\sim 5$, comparable to that in aqueous solution, at the very early step of ionization, it is clear that the low dielectric constant of these monolayer does not affect this ionization very much ${ }^{51}$. As the extent of ionization increases from 0.4 to 1.0 , the effective $\mathrm{pK}_{\mathrm{a}}$ of the hydrogen-bonded dimeric acid increases from 5 to 10 . This increase in the effective $\mathrm{pK}_{\mathrm{a}}$ is comparable to that for polyethylene carboxylic acid studied by Whitesides and co-workers ${ }^{51}$. They believed that the increase in the effective $\mathrm{pK}_{\mathrm{a}}$ might reflect either a change in the ease of ionization of a homogeneous population of carboxylic acid groups as the electrically neutral carboxylic acid groups are converted to anionic carboxylate groups or heterogeneity in the population of carboxylic acid groups being examined. We believe that it is the electrostatic repulsion but not the dielectric constant that plays the most important role for the acid-base chemistry of these acidterminated alkanethiol monolayers.

In these analysis, the error of the values of the effective $\mathrm{pK}_{\mathrm{a}}$ are too 


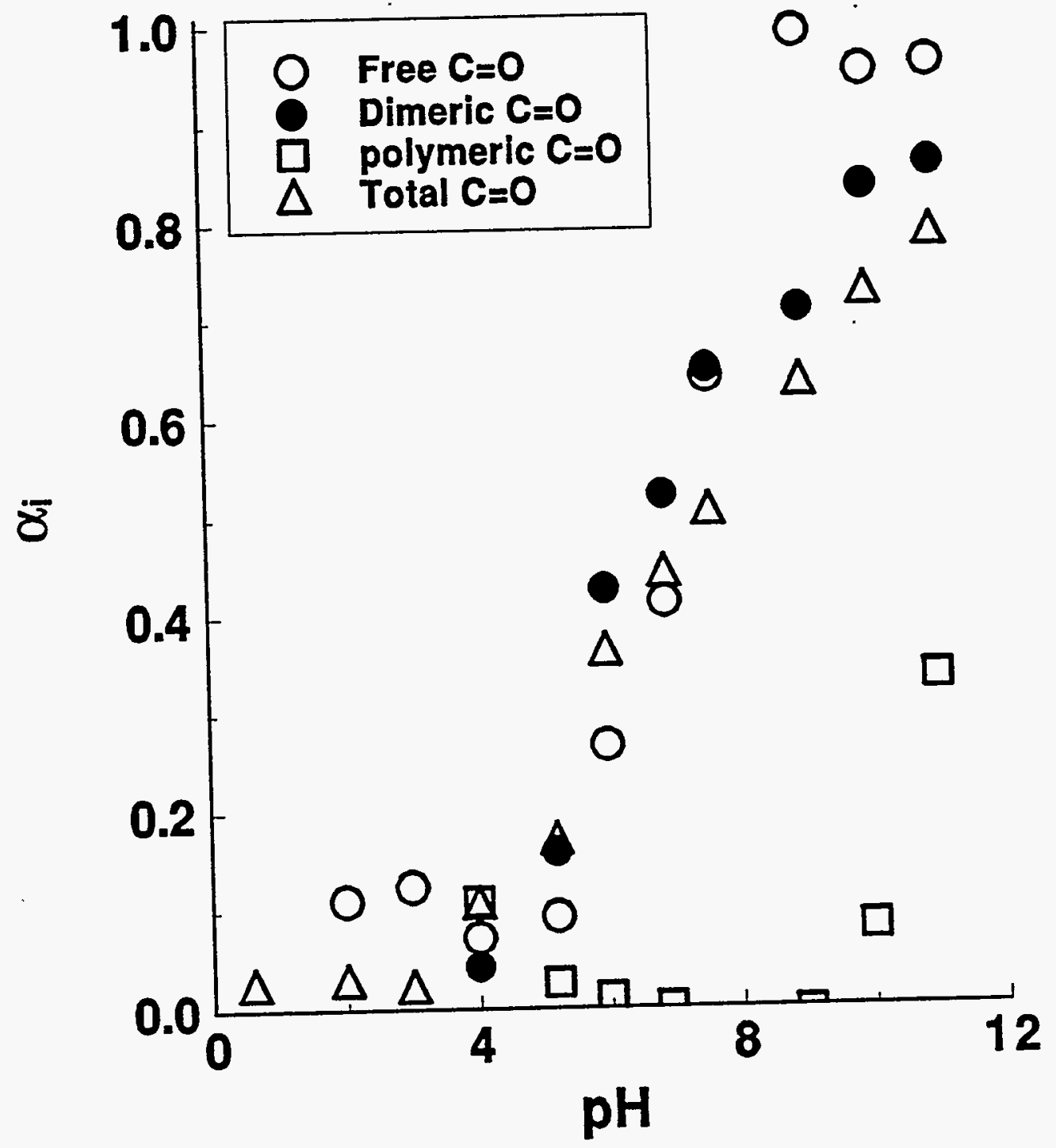

Figure 12. The degree of ionization $\alpha_{\mathrm{i}}$ of a $\mathrm{HO}_{2} \mathrm{C}\left(\mathrm{CH}_{2}\right)_{15} \mathrm{~S} / \mathrm{Au}$ monolayer with a fraction coverage of 0.65 as a function of solution $\mathrm{pH}$. 
large to be meaningful when $\alpha_{i}$ is either smaller than 0.1 or greater than 0.9. The values of effective $\mathrm{pK}_{\mathrm{a}}$ for both the free acid and the dimeric acid are about the same and have a constant value of 5.0 when the extent of ionization is less than 0.4 . It is very clear that the effective $\mathrm{pK}_{\mathrm{a}}$ of hydrogen-bonded polymeric acid is around 11. Interestingly, the effective $\mathrm{pK}_{\mathrm{\alpha}}$ of the total acid is almost the same as that of the hydrogenbonded dimeric acid. We will see the same trend for different monolayers as well. The effective $\mathrm{pK}$, values at $50 \%$ of ionization for the free acid, the hydrogen-bonded dimeric acid, the hydrogen-bonded polymeric acid, and the overall are $6.9,7.0,11$, and 7.2 , respectively.

\section{MHA monolayers with a full coverage}

No noticeable change in the IRRAS spectra of $v\left(\mathrm{CH}_{2}\right)$ modes in Figures 14 for a MHA/Au prepared in $0.10 \mathrm{mM}$ solution for 24 hours as a function of solution $\mathrm{pH}$ indicates that the orientation of the polymethylene chain remains the same through out this $\mathrm{pH}$ windows studied. The IRRAS spectra of $v(\mathrm{C}=0)$ modes of the same monolayer as a function of solution $\mathrm{pH}$ are shown in Figure 15. As you can see, the $v(C=0)$ peaks of this monolayer are quite different from the MHA/Au monolayer with a fractional coverage of 0.65 . These $v(C=0)$ peaks have $\sim 20 \%$ of the free $v(\mathrm{C}=0), \sim 20 \%$ of the hydrogen-bonded polymeric $v(\mathrm{C}=0)$, and only 60 $\%$ of the hydrogen-bonded dimeric $v(\mathrm{C}=\mathrm{O})$. All the amount of these acids decrease with increasing the solution $\mathrm{pH}$ and disappear when the solution $\mathrm{pH}$ is greater than 11 . The recovery of these acids after re-immersion of these monolayers back into $\mathrm{pH} 1$ solution suggests that not only there is virtually no change in the orientation of the film but also there is almost no loss of these acid monolayers during the ionization process. There is a slight increase in the amount of the hydrogen-bonded dimeric acid after it is re-immersed.

This monolayer has three different acid groups behavior similar to that of the monolayer with a fractional coverge of 0.65 although there is a difference in the peak area as shown in Figure 16. The free acid starts to 


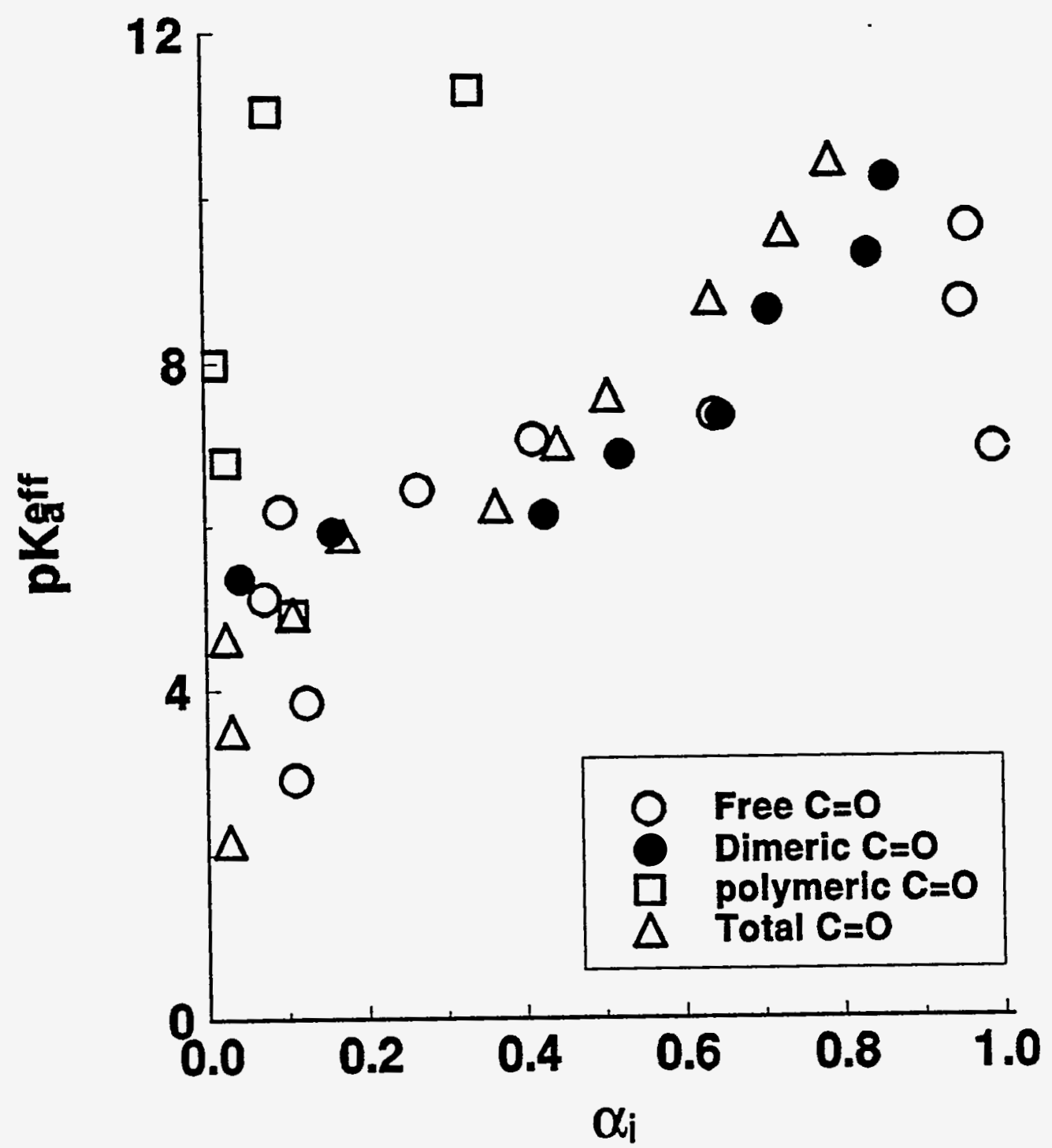

Figure 13. The effective $\mathrm{pK}_{\mathrm{a}}$ of a $\mathrm{HO}_{2} \mathrm{C}\left(\mathrm{CH}_{2}\right)_{15} \mathrm{~S} / \mathrm{Au}$ monolayer with a fraction coverage of 0.65 as a function of the extent of ionization $\alpha_{\mathrm{i}}$. 
ionize at $\mathrm{pH} 4$ and almost completes the ionization process at $\mathrm{pH} 8$. The hydrogen-bonded dimeric acid starts to ionize at $\mathrm{pH} 4$ and has finished by $\mathrm{pH}$ 11. The hydrogen-bonded polymeric acid remains intact until $\mathrm{pH} 10$. The $\mathrm{pK}_{\mathrm{a}}$ for the non-hydrogen-bonded acid, the hydrogen-bonded dimeric acid, the hydrogen-bonded polymeric acid, and the overall $\mathrm{pK}_{\mathrm{a}}$ are 6.5, $7.0,11.0$, and 7.2, respectively.

The calculated values of the extent of ionization for all these acid groups are shown in Figure 17. It is clear that the free acid has the highest value at all $\mathrm{pH}$ and virtually reaches a value of 1 when the solution $\mathrm{pH}$ is greater than 8 . The extent of ionization of the dimeric acid has a value higher than that of polymeric acid but smaller than that of free acid. The extent of ionization of the hydrogen-bonded polymeric acid not only has the smallest value and very little till $\mathrm{pH} 8$. This clearly demonstrates that these three different acid groups have remarkably different properties as far as the extent of ionization is concerned. That is, in terms of acidity: the free acid $>$ the hydrogen-bonded dimeric acid $>$ the hydrogen-bonded polymeric acid.

Obviously, the effective $\mathrm{pK}_{\mathrm{a}}$ of these three different acid groups behave very differently as shown in Figure 18 . The effective $\mathrm{pK}_{\mathrm{a}}$ of the free acid has a value of 5 at the beginning of ionization and increases to $\sim 7$ when the free acid is almost fully ionized. The effective $\mathrm{pK}_{\mathrm{a}}$ of the hydrogen-bonded dimeric acid has a initial value of $\sim 6$ and increases to $\sim 8$. The effective $\mathrm{pK}_{\mathrm{a}}$ of hydrogen-bonded polymeric acid is $\sim 10$. Surprisingly, the effective $\mathrm{pK}_{\mathrm{z}}$ of the overall acid group has almost the same value as that of the hydrogen-bonded dimeric acid again. At $50 \%$ of ionization, the effective $\mathrm{pK}_{2}$ values of the free acid, the hydrogenbonded dimeric acid, the hydrogen-bonded polymeric acid, and the overall value are $6.5,7.8,11.0$ and 7.8 , respectively. So, it is clear that these three acids have significant differences in their acid properties. Interestingly, the overall properties of these three acids are virtually the same as that of the hydrogen-bonded dimeric acid. 


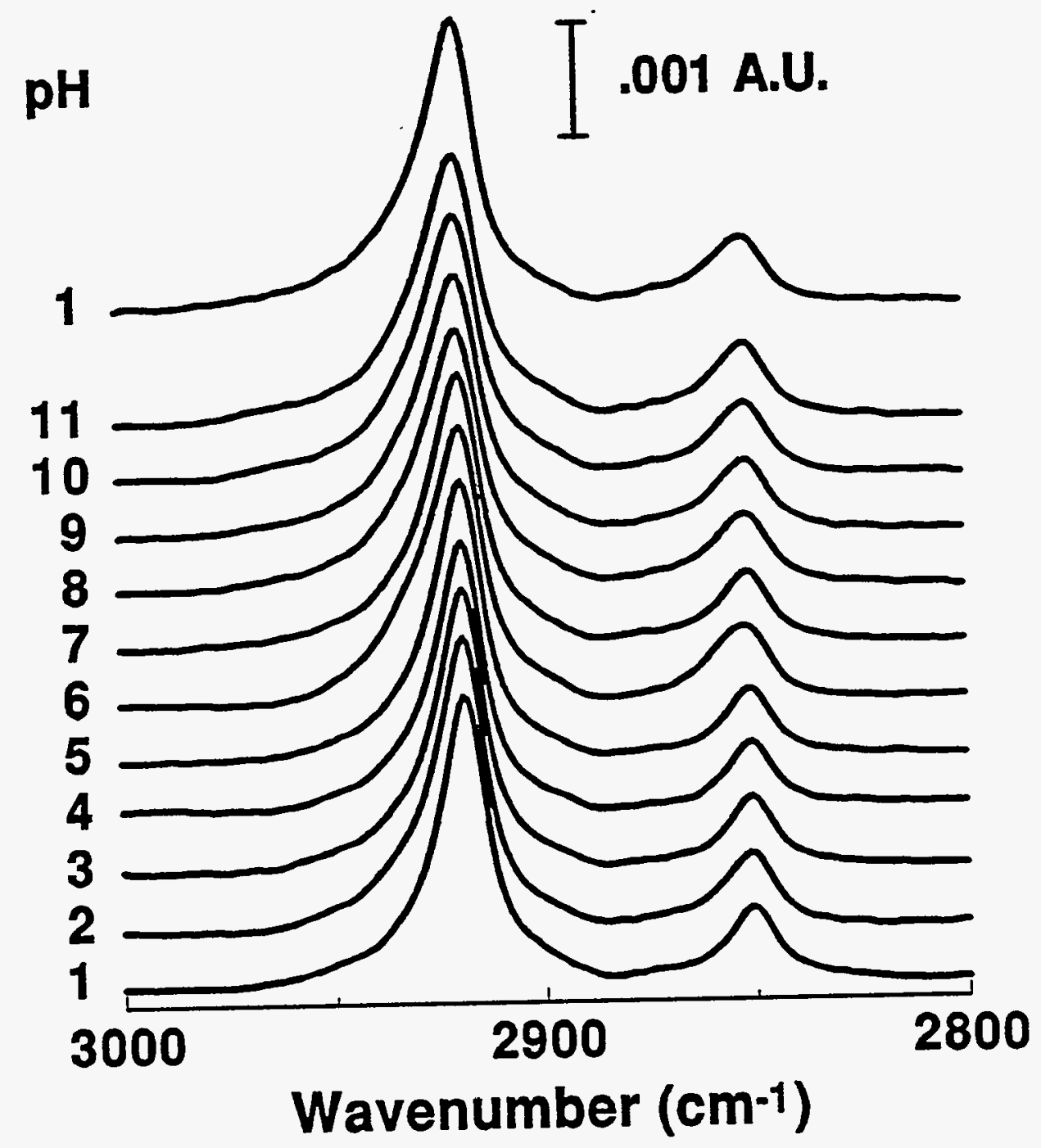

Figure 14. IRRAS spectra in high energy region for a $\mathrm{HO}_{2} \mathrm{C}\left(\mathrm{CH}_{2}\right)_{15} \mathrm{~S} / \mathrm{Au}$ monolayer as a function of solution $\mathrm{pH}$. 


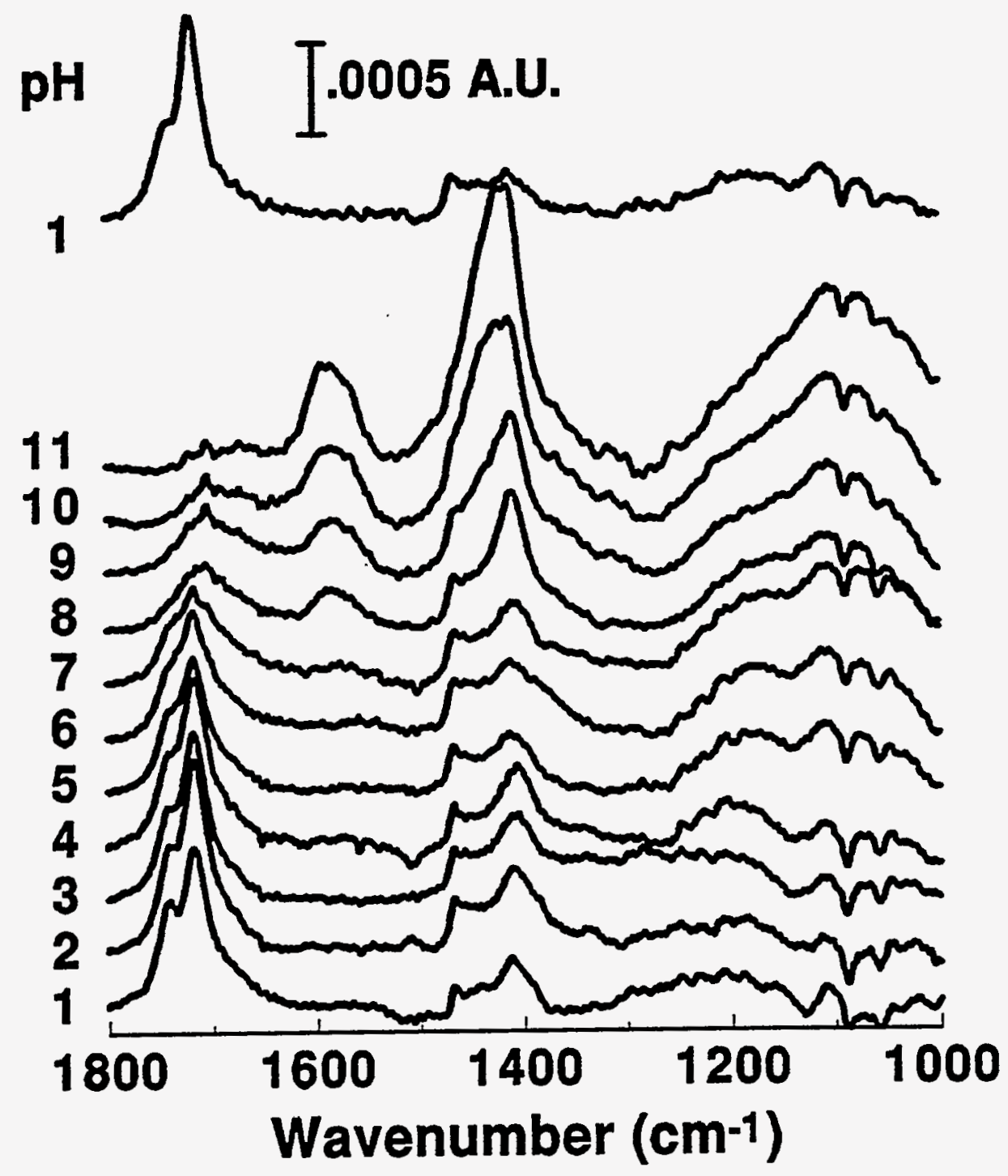

Figure 15. IRRAS spectra in low energy region for a $\mathrm{HO}_{2} \mathrm{C}\left(\mathrm{CH}_{2}\right)_{15} \mathrm{~S} / \mathrm{Au}$ monolayer hours as a function of solution $\mathrm{pH}$. 


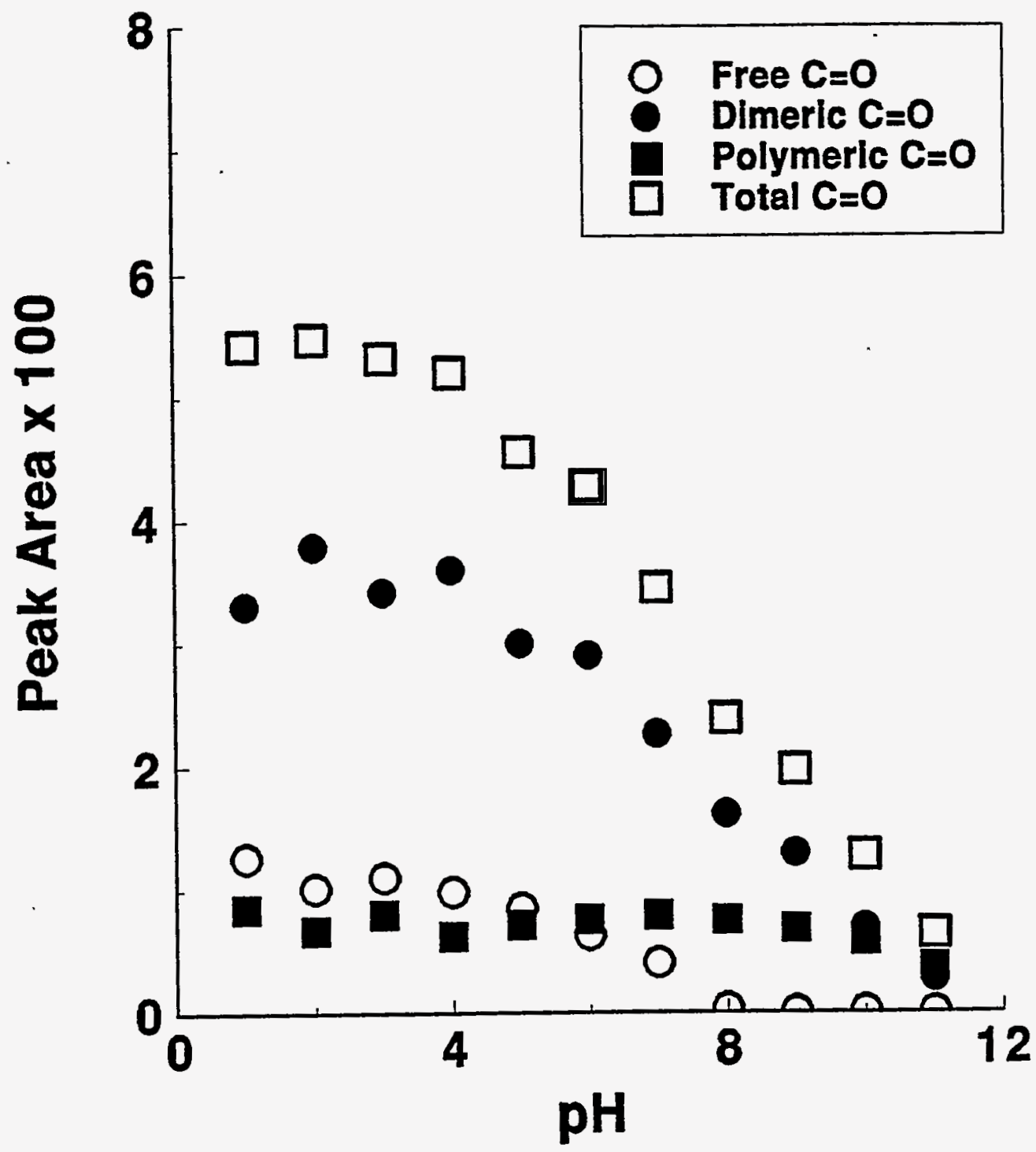

Figure 16. The IRRAS peak area of the $C=O$ peaks of a $\mathrm{HO}_{2} \mathrm{C}\left(\mathrm{CH}_{2}\right)_{15} \mathrm{~S} / \mathrm{Au}$ monolayer as a function of solution $\mathrm{pH}$. 


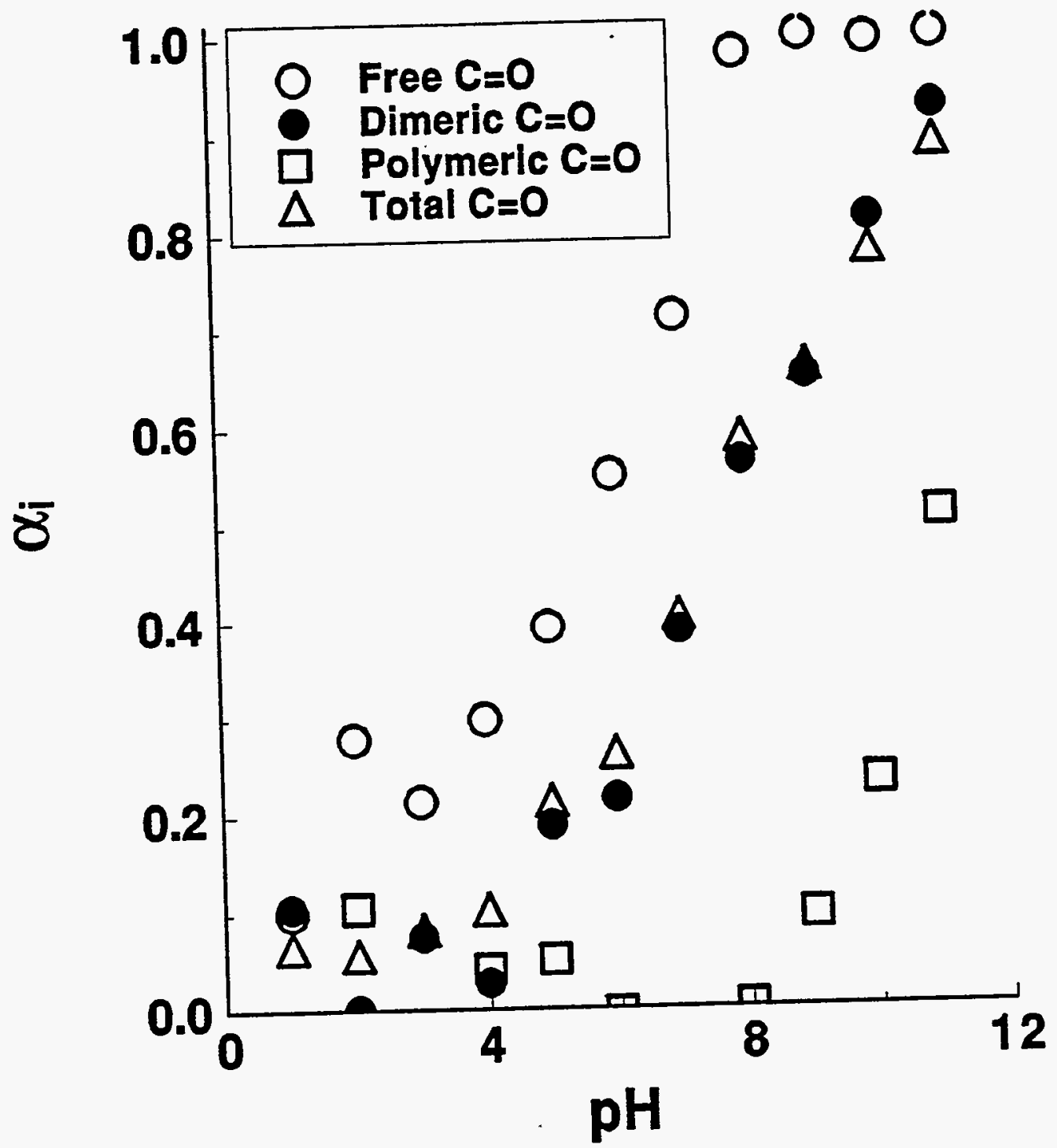

Figure 17. The degree of ionization $\alpha_{\mathrm{i}}$ of a $\mathrm{HO}_{2} \mathrm{C}\left(\mathrm{CH}_{2}\right)_{15} \mathrm{~S} / \mathrm{Au}$ monolayer as a function of solution $\mathrm{pH}$. 
The comparison between the effective $\mathrm{pK}_{\mathrm{a}}$ for the $\mathrm{MHA} / \mathrm{Au}$ monolayer with a fractional coverge of 0.65 and 0.98 shows not much difference between these two monolayers although there is a slightly higher value for free acid of the monolayer with a fraction coverage of 0.65 . The slight difference most likely is due to the detection limit of the very small amount of the free acid in the monolayer.

\section{MUA monolavers}

No change in the $v\left(\mathrm{CH}_{2}\right)$ modes in Figure 19 for a 24-hourimmersed MUA/Au monolayer indicates the conservation of the orientation of the polymethylene chain of this monolayer. The recovery of all these three $v(\mathrm{C}=0)$ modes in Figure 20 after re-immersion back in $\mathrm{pH}$ 1 solution confirms this point. It is apparent that there is a slight increase in the amount of the hydrogen-bonded dimeric acid after it is re-immersed.

In this film, the relative amount of the free acid, the hydrogenbonded polymeric acid, and the hydrogen-bonded dimeric acid are $25 \%$, $25 \%$, and $50 \%$, respectively, as shown in Figure 21. This indicates that in this monolayer, more hydrogen-bonded dimeric acids are converted to both the free and hydrogen-bonded polymeric acids than a MHA/Au monolayer after 24 hours immersion time in $0.1 \mathrm{mM}$ solution. The peak area of the free $\mathrm{v}(\mathrm{C}=\mathrm{O})$ mode starts decreasing at $\mathrm{pH} 5$ and disappears at $\mathrm{pH} 8$. The peak area of the hydrogen-bonded dimeric $v(\mathrm{C}=0)$ mode starts decreasing at $\mathrm{pH} 4$ and keeps decreasing until the solution $\mathrm{pH}$ is greater than 11. Clearly, the peak area of the hydrogen-bonded polymeric $v(\mathrm{C}=\mathrm{O})$ mode is constant until the solution $\mathrm{pH}$ is greater 9 . The calculated $\mathrm{pK}_{\mathrm{a}}$ values for the free acid, the dimeric acid, the polymeric acid, and the overal $\mathrm{pK}_{\mathrm{a}}$ are $6.1,7.8,10.5$ and 8.0, respectively. The transition range of the free acid, the dimeric acid, and the polymeric acid are 3, 7 and $3 \mathrm{pH}$ units, respectively. $\mathrm{By} \mathrm{pH} 8$, the peak area of the free acid disappears completely while that of polymeric acid remains almost intact. 


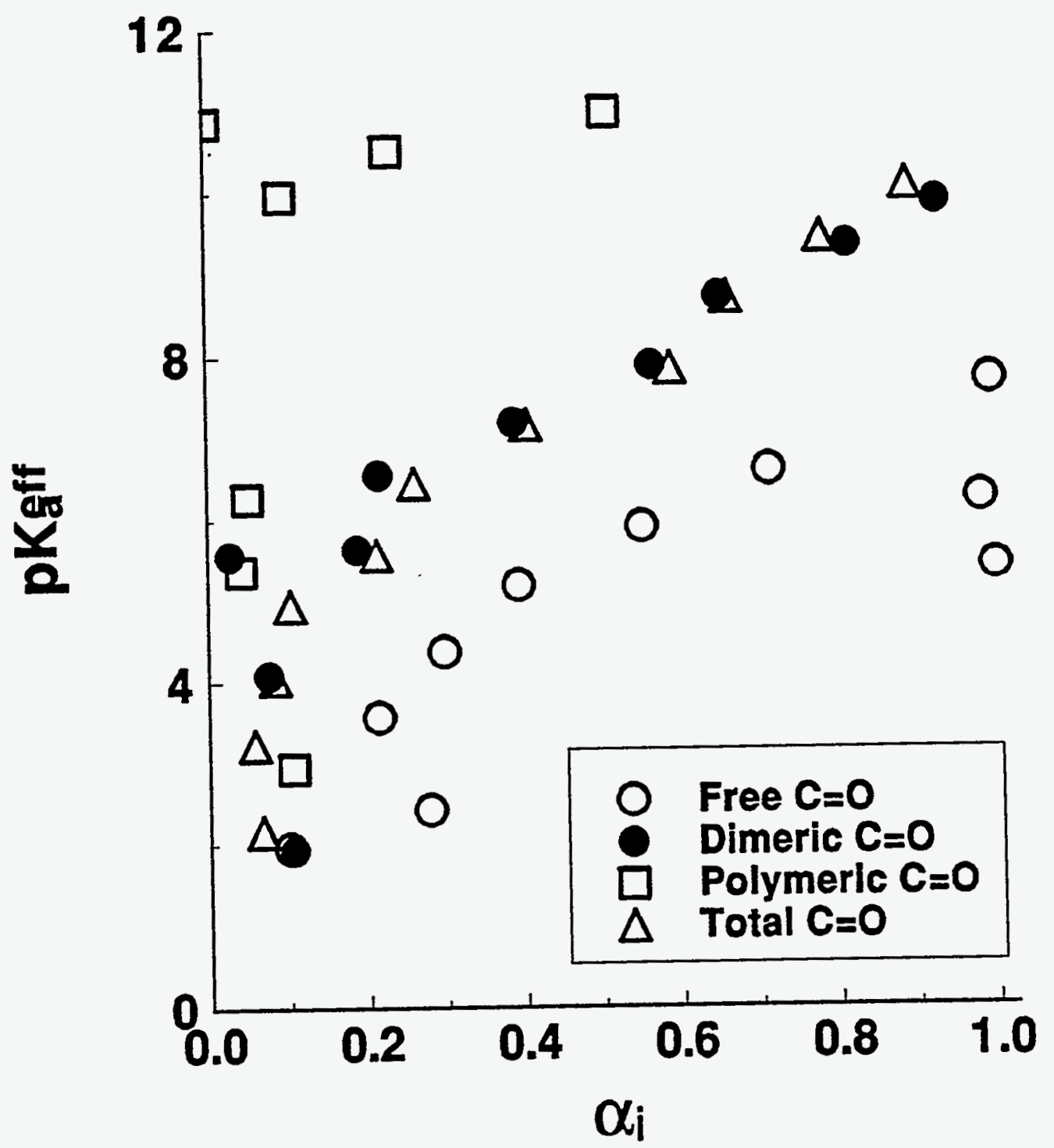

Figure 18. The effective $\mathrm{pK}_{\mathrm{a}}$ of a $\mathrm{HO}_{2} \mathrm{C}\left(\mathrm{CH}_{2}\right)_{15} \mathrm{~S} / \mathrm{Au}$ monolayer as a function of the extent of ionization $\alpha_{i}$. 
The extent of ionization as a function of solution $\mathrm{pH}$ is shown in Figure 22 which shows almost the same trend as that for the MHA/Au monolayer in Figures 12 and 17. Once again, the free acid has the highest values at almost any $\mathrm{pH}$ and the hydrogen-bonded polymeric acid has the lowest values with the hydrogen-bonded dimeric acid in between. The free acid starts to ionize at $\mathrm{pH} 5$ and almost completes at $\mathrm{pH} 8$. The hydrogen-bonded dimeric acid starts to ionize at $\mathrm{pH} 4$ and barely finishes at $\mathrm{pH} 11$. The hydrogen-bonded polymeric acid remains almost intact until pH 9. The overall performance is basically the same as that of the hydrogen-bonded dimeric acid.

The values of the effective $\mathrm{pK}$, for all these acid groups as a function of the extent of ionization are shown in Figure 23. The free acid has an initial value of $\sim 5$ and increases up to $\sim 8$. The hydrogen-bonded dimeric acid with a initial value of $\sim 5$ increases with increasing the extent of ionization and levels off with a finial value of $\sim 10$. The value for the hydrogen-bonded polymeric acid is $\sim 10$ though there is only one data point. Surprisingly, the overall values are very close to that of the hydrogen-bonded dimeric acid again. The effective $\mathrm{pK}_{\mathrm{z}}$ values at $50 \%$ of ionization for free acid, the hydrogen-bonded dimeric acid, the hydrogenbonded polymeric acid, and the overall are $6.3,8.3,11.0$, and 8.3, respectively.

The comparison between the effective $\mathrm{pK}_{\mathrm{z}}$ values of $\mathrm{MUA} / \mathrm{Au}$ with that of MHA/Au monolayer shows a slightly higher values for MUA/Au monolayer by $\sim 10 \%$ in the overall effective $\mathrm{pK}$, value although there is not much difference in the beginning of ionization. Both monolayers have almost the same effective $\mathrm{pK}$, values for both the free acid and the polymeric acid, but the MUA/Au monolayer has a higher value for the dimeric acid particularly at high extent of ionization. These higher effective $\mathrm{pK}_{\mathrm{a}}$ values in MUA/Au monolayers most likely is because the MUA/Au monolayer has higher packing density than MHA/Au monolayer. If this is true, then this further confirms that the electrostatic repulsion is the dominant factor in this ionization process. 


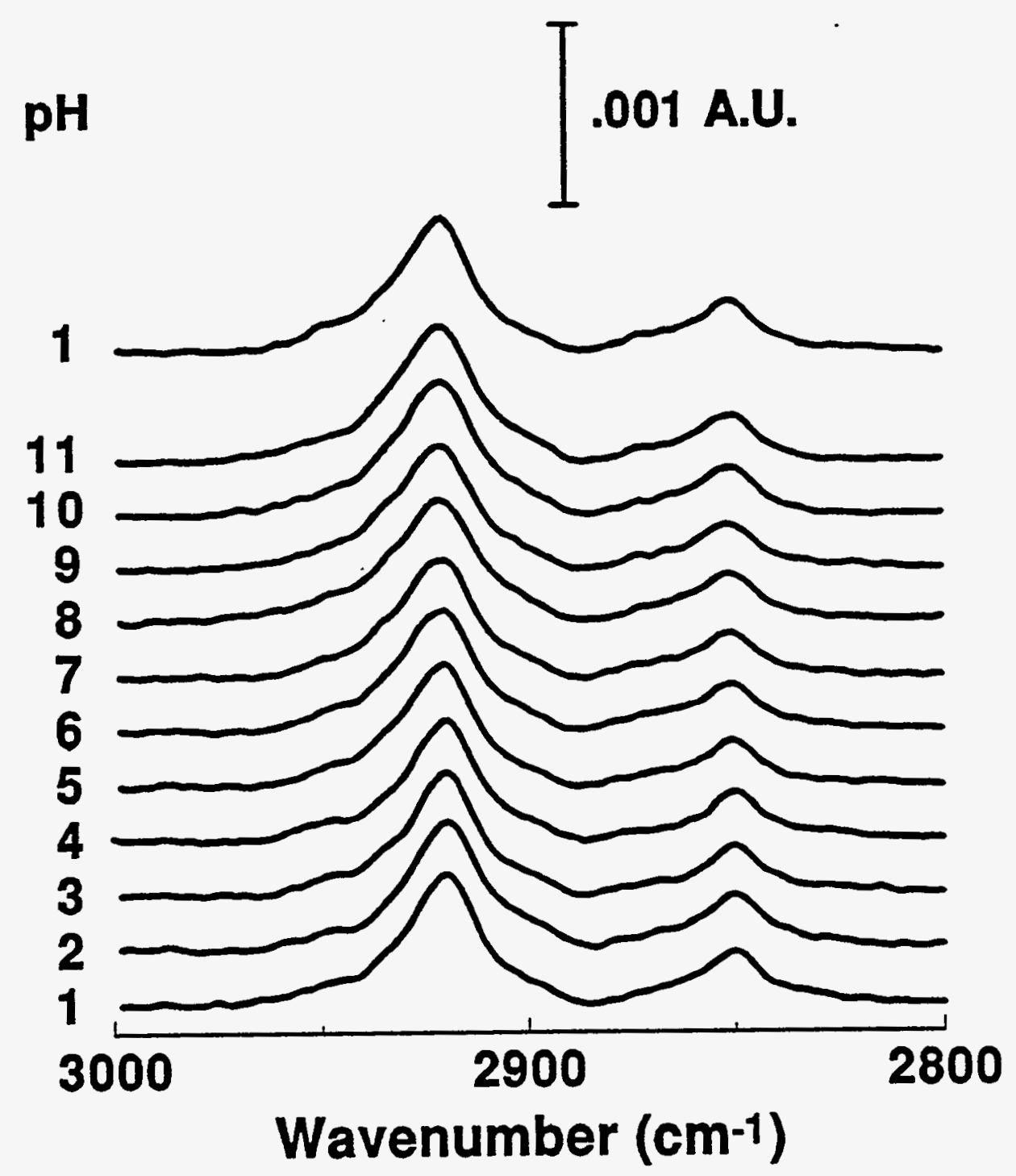

Figure 19. IRRAS spectra in high energy region of a $\mathrm{HO}_{2} \mathrm{C}\left(\mathrm{CH}_{2}\right)_{10} \mathrm{~S} / \mathrm{Au}$ monolayer as a function of solution $\mathrm{pH}$. 


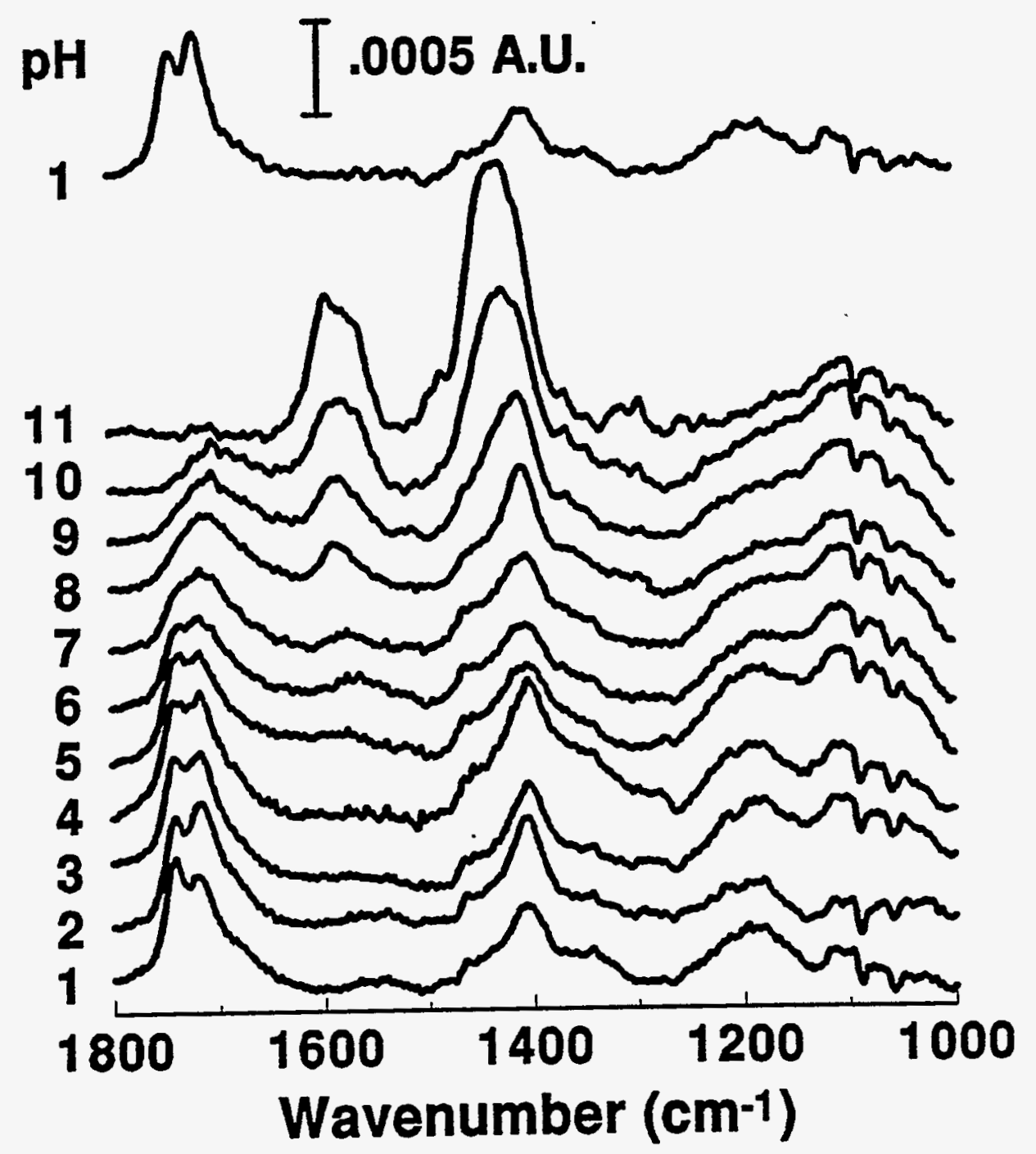

Figure 20. IRRAS spectra in low energy region for a $\mathrm{HO}_{2} \mathrm{C}\left(\mathrm{CH}_{2}\right)_{10} \mathrm{~S} / \mathrm{Au}$ monolayer as a function of solution $\mathrm{pH}$. 


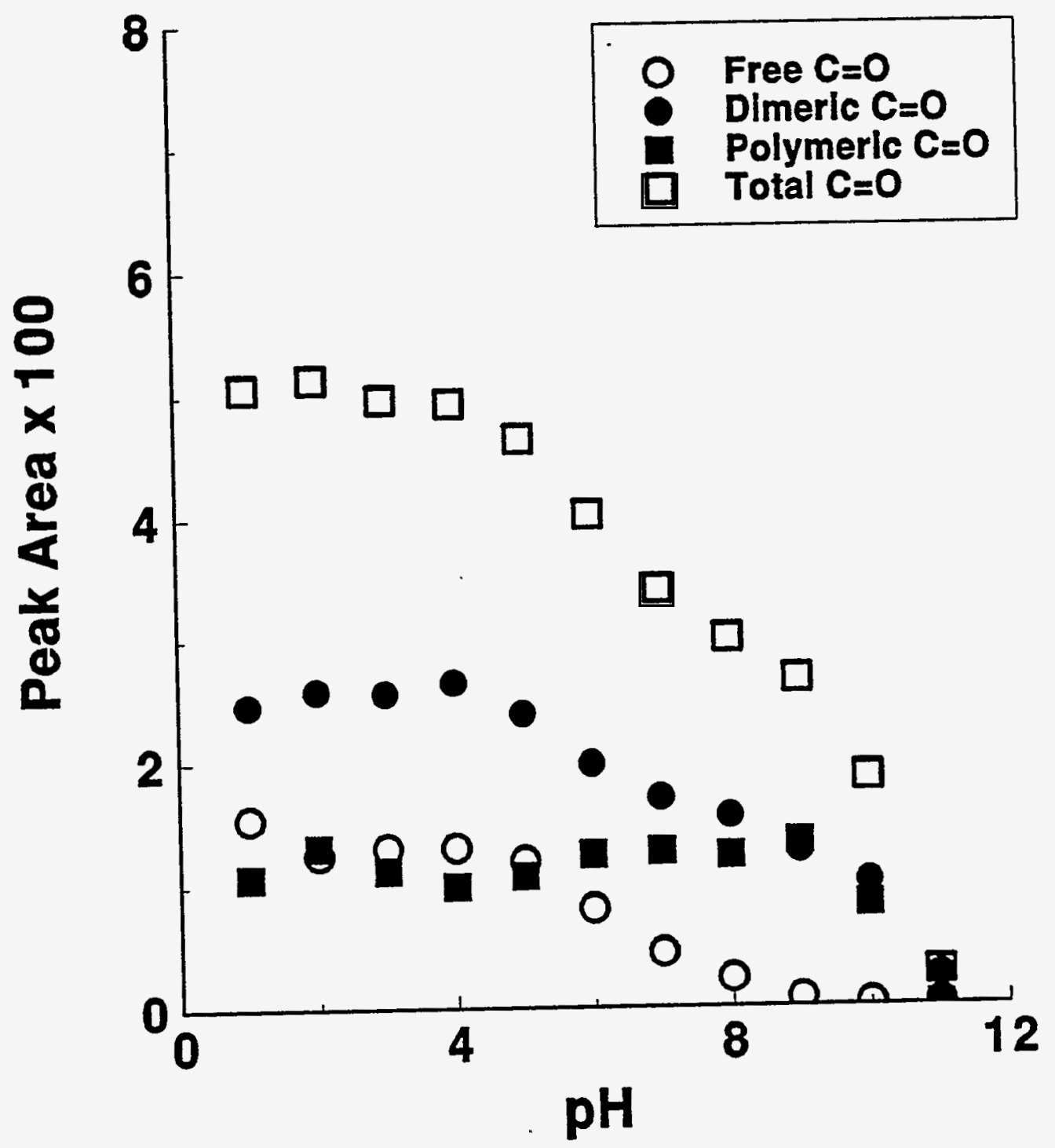

Figure 21. The IRRAS peak area of the $\mathrm{C}=\mathrm{O}$ peaks of a $\mathrm{HO}_{2} \mathrm{C}\left(\mathrm{CH}_{2}\right)_{10} \mathrm{~S} / \mathrm{Au}$ monolayer as a function of solution $\mathrm{pH}$. 


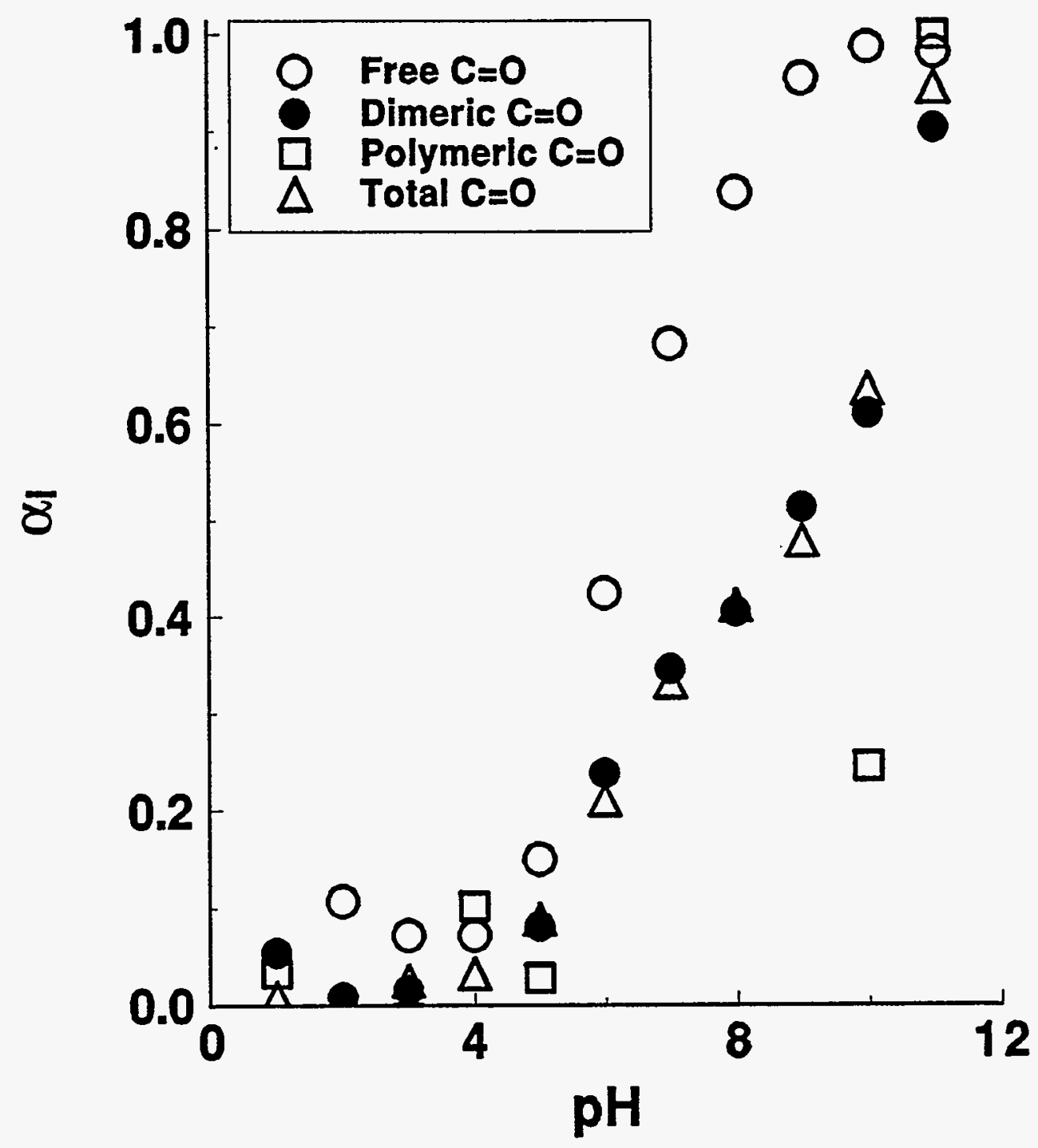

Figure 22. The degree of ionization $\alpha_{\mathrm{i}}$ of a $\mathrm{HO}_{2} \mathrm{C}\left(\mathrm{CH}_{2}\right)_{10} \mathrm{~S} / \mathrm{Au}$ monolayer as a function of solution $\mathrm{pH}$. 


\section{MPA monolayers}

There is virtually no $v\left(\mathrm{CH}_{2}\right)$ peaks for the MPA/Au monolayer as shown in Figure 24. This indicate that axis of the two $\mathrm{CH}_{2}$ groups are almost parallel to the surface normal so that the plane of each $\mathrm{CH}_{2}$ is parallel to the surface. No change in this IRRAS region during the ionization process implies this chain axis remains the same all the time. This is further supported by the full recovery of the all the IRRAS peaks by re-immersion back to $\mathrm{pH} 0.50$ solution as shown in Figure 25.

Both the $v_{\mathrm{a}}\left(\mathrm{COO}^{-}\right)$and $v_{\mathrm{s}}\left(\mathrm{COO}^{-}\right)$peaks do not appear until $\mathrm{pH} \sim 6$ where the $v(C=O)$ peaks almost disappear as shown in Figures 25 and 26.

The peak area of the three $v(C=0)$ peaks as a function of solution $\mathrm{pH}$ are shown in Figure 27. Apparently, the free $v(\mathrm{C}=0)$ starts to be ionized after $\mathrm{pH} 3.5$ and finishes the ionization process by $\mathrm{pH} 7.5$. The hydrogen-bonded dimeric $v(\mathrm{C}=\mathrm{O})$ starts ionization around $\mathrm{pH} 2$ all the way up to $\mathrm{pH} 11$. This transition of $9 \mathrm{pH}$ units is very broad. The hydrogen-bonded polymeric $v(\mathrm{C}=\mathrm{O})$ dose not have any significant ionization until $\mathrm{pH} 7$.

With an initial value of about 0.05 at $\mathrm{pH} 3$, the extent of ionization for both the free $\mathrm{C}=\mathrm{O}$ and the hydrogen-bonded dimeric $\mathrm{C}=\mathrm{O}$ starts to increase all the way to a finial value of 1 by $\mathrm{pH} 8$ and $\mathrm{pH} 11$, respectively, as shown in Figure 28. Meanwhile, the value for the hydrogen-bonded polymeric $\mathrm{C}=\mathrm{O}$ is virtually 0 until around $\mathrm{pH} 7$. The values of the effective $\mathrm{pK}_{\mathrm{a}}$ for all three acid groups as a function of the extent of ionization are shown in Figure 29. The effective $\mathrm{pK}_{\mathrm{a}}$ of the free acid always has the lowest value, although it is not very clear due to the too small amount of this free acid present in this monolayer. The effective $\mathrm{pK}_{\mathrm{\alpha}}$ of the free acid has an initial value of $\sim 4.5$ and remains almost the same value till $50 \%$ of ionization and starts to increase to a finial value of $\sim 8$. The effective $\mathrm{pK}$, of the hydrogen-bonded dimeric acid has an initial value of 4.5 and increases up to $\sim 10$. It is clear that the has a initial value of about 8 and increase up to 11 , though the ionization is only $66 \%$ 


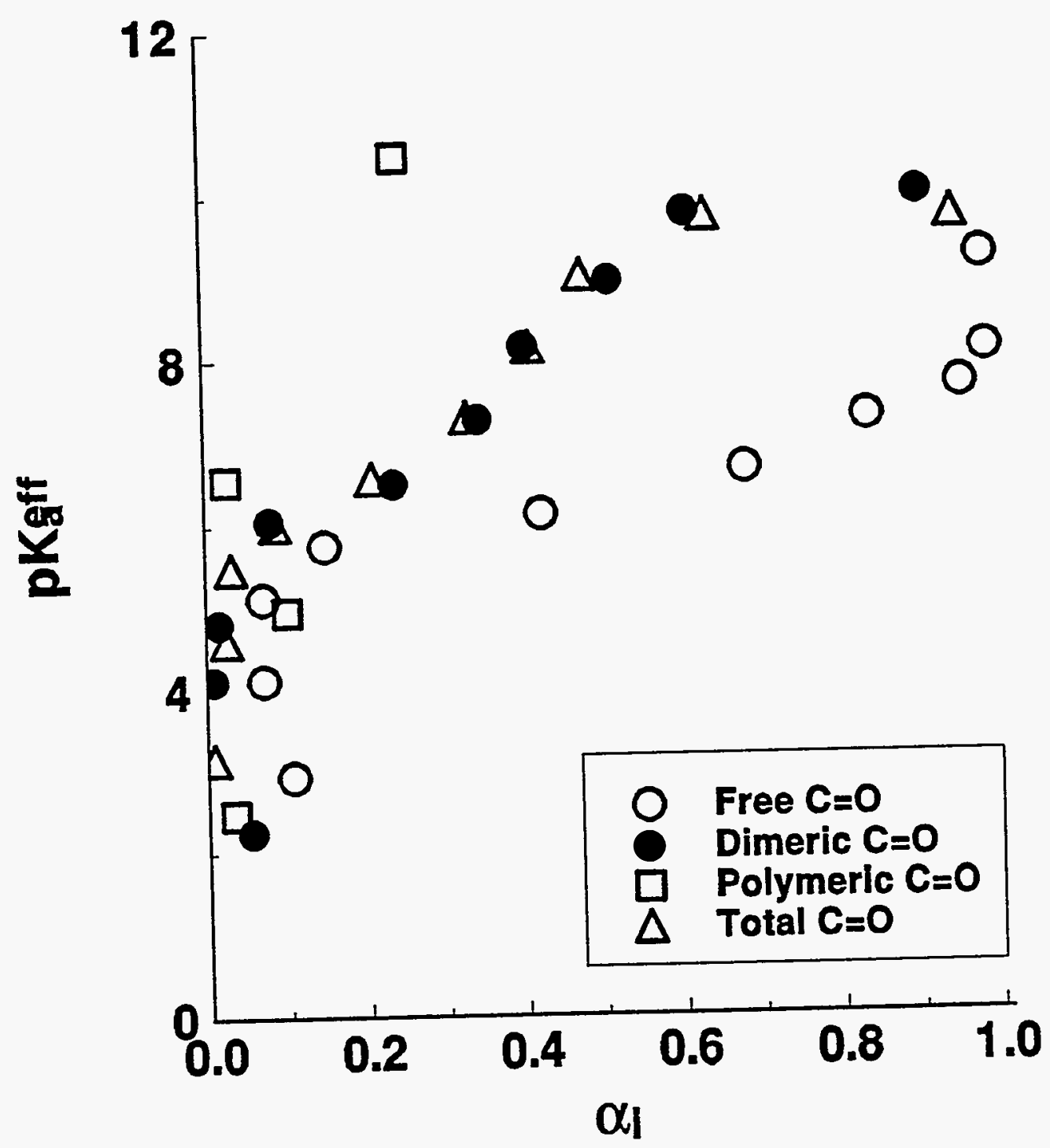

Figure 23. The effective $\mathrm{pK}_{\mathrm{a}}$ of $\mathrm{HO}_{2} \mathrm{C}\left(\mathrm{CH}_{2}\right)_{10} \mathrm{~S} / \mathrm{Au}$ monolayer as a function of the extent of ionization $\alpha_{\mathrm{i}}$. 
remain the same or decrease but not to increase as increasing solution $\mathrm{pH}$ as seen for all the monolayers mentioned previously. In this disulfide monolayer, there is a free space between two neighbor $\mathrm{C}=0$ groups since the alkane chain is shorter by $6 \mathrm{CH}_{2}$ in length. This provides the room for those carboxylate group which has one negative charge to eliminate the strong electrostatics repulsion. This suggests that the carboxylate group like to turn around to minimize the electrostatic repulsion if possible. Therefore, the two peaks cannot be used for the calculation of the extent complete. The large deviation for hydrogen-bonded polymeric acid is due to the very weak signal for it. The overall effective $\mathrm{pK}_{\mathrm{a}}$ is almost has the same values as that for the hydrogen-bonded dimeric acid. The effective $\mathrm{pK}_{\mathrm{z}}$ values at $50 \%$ of ionization for the free acid, the hydrogen-bonded dimeric acid, the hydrogen-bonded polymeric acid, and the overall are $5.3,5.7,9.5$, and 6.0 , respectively.

\section{PUAD monolayers}

Unlike carboxylic acid-terminated thiolate monolayers, the PUAD/Au monolayer has a change in the $v(\mathrm{C}-\mathrm{H})$ peaks intensity of the IRRAS spectra during the ionization process as shown in Figure 30. The peak intensity at $2926 \mathrm{~cm}^{-1}$ almost has a same value of $0.0010 \mathrm{~A}$.U. when $\mathrm{pH}$ is less than 4 and starts increasing and levels off with a limiting value of 0.0018 A.U. by pH 6 as shown in Figure 31. Upon ionization, the peak intensity of the $v(\mathrm{C}-\mathrm{H})$ mode at $2926 \mathrm{~cm}^{-1}$ jumps from 0.0010 to 0.0018 A.U. as shown in Figure 32. Apparently, the monolayer structure has been disturbed by the electrolyte and the buffer after it is ionized. This is further supported by the change in the $v(\mathrm{C}=0)$ peaks after reimmersion back to $\mathrm{pH} 1$ solution and the presence of the extra phosphate (as a buffer) peaks around $1110 \mathrm{~cm}^{-1}, 1265 \mathrm{~cm}^{-1}$, and $1410 \mathrm{~cm}^{-1}$ as shown in Figure 33. The incorporation of the electrolyte and the buffer into this monolayer after ionization can be confirmed further from the increase in the peak area of the free $v(\mathrm{C}=\mathrm{O})$ mode and the hydrogenbonded polymeric $v(\mathrm{C}=\mathrm{O})$ mode with increasing the extent of ionization as shown in Figure 34. These two peak areas are supposed to either 


\section{$\mathrm{Au}-\mathrm{S}(\mathrm{CH} 2) 2 \mathrm{COOH}$ at $0.60 \mathrm{pH}$}
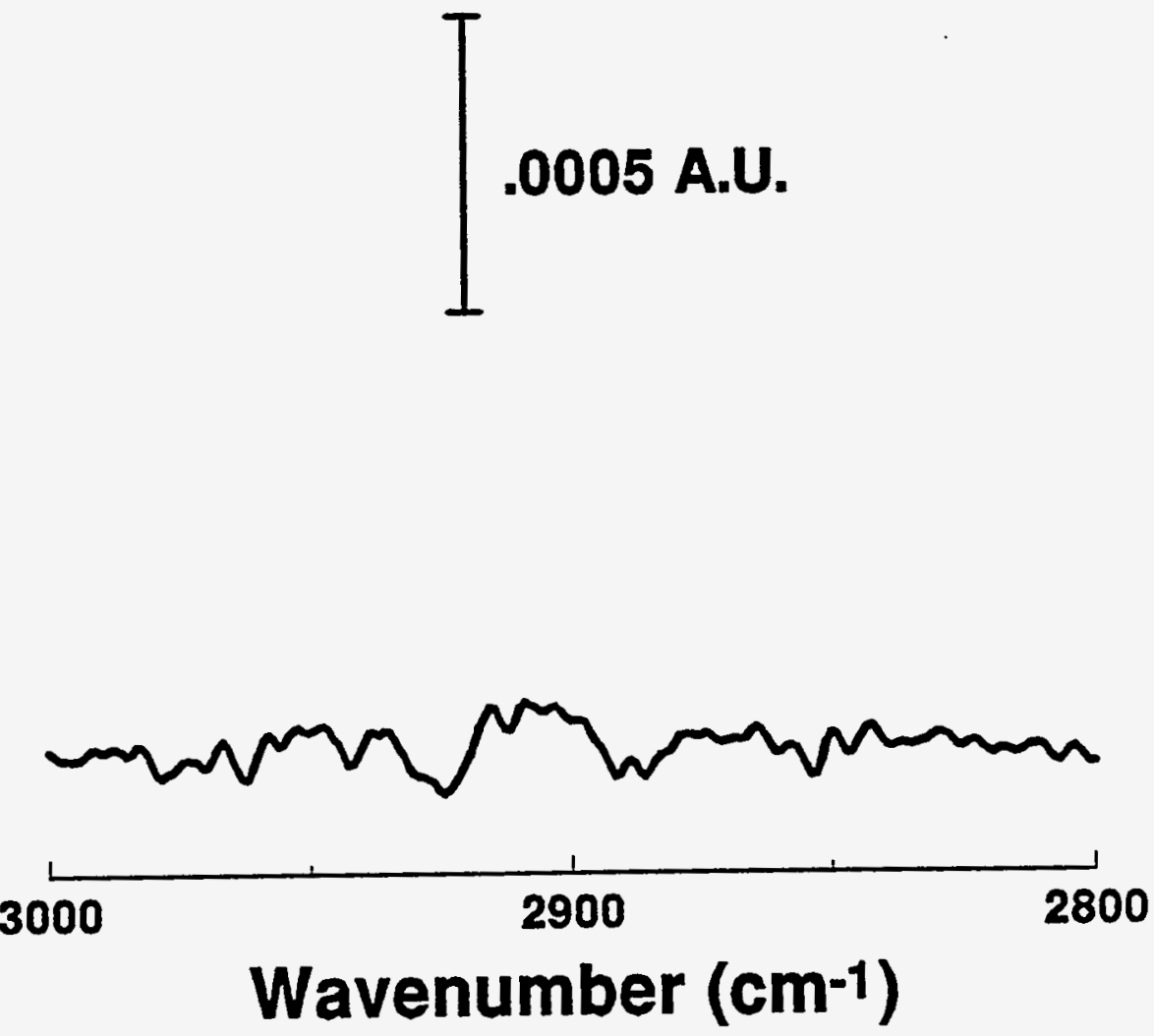

Figure 24. IRRAS spectra in the high energy region of a $\mathrm{HO}_{2} \mathrm{C}\left(\mathrm{CH}_{2}\right)_{2} \mathrm{~S} / \mathrm{Au}$ monolayer as a function of solution $\mathrm{pH}$. 


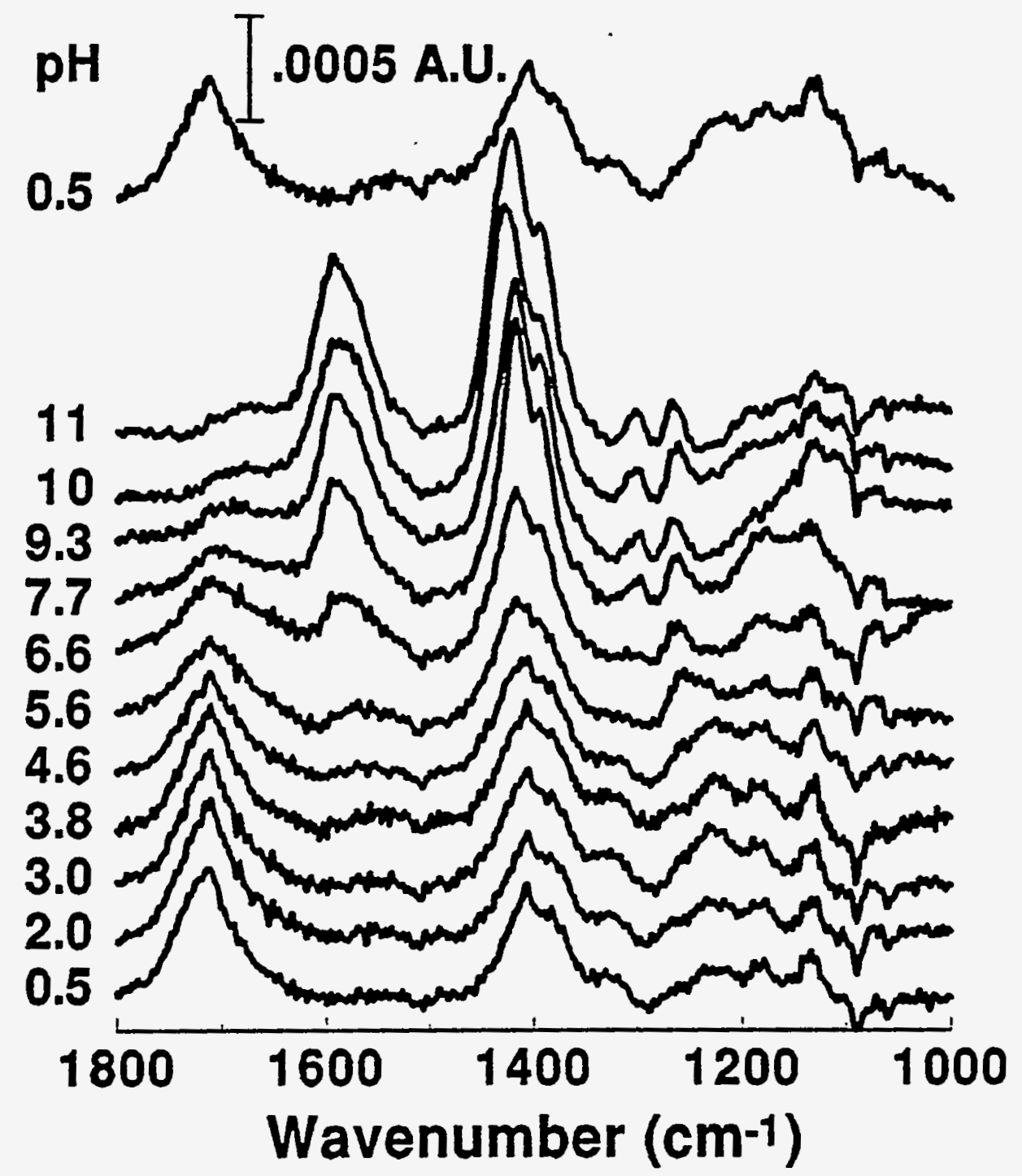

Figure 25. IRRAS spectra in the low energy region for a $\mathrm{HO}_{2} \mathrm{C}\left(\mathrm{CH}_{2}\right)_{2} \mathrm{~S} / \mathrm{Au}$ monolayer as a function of solution $\mathrm{pH}$. 

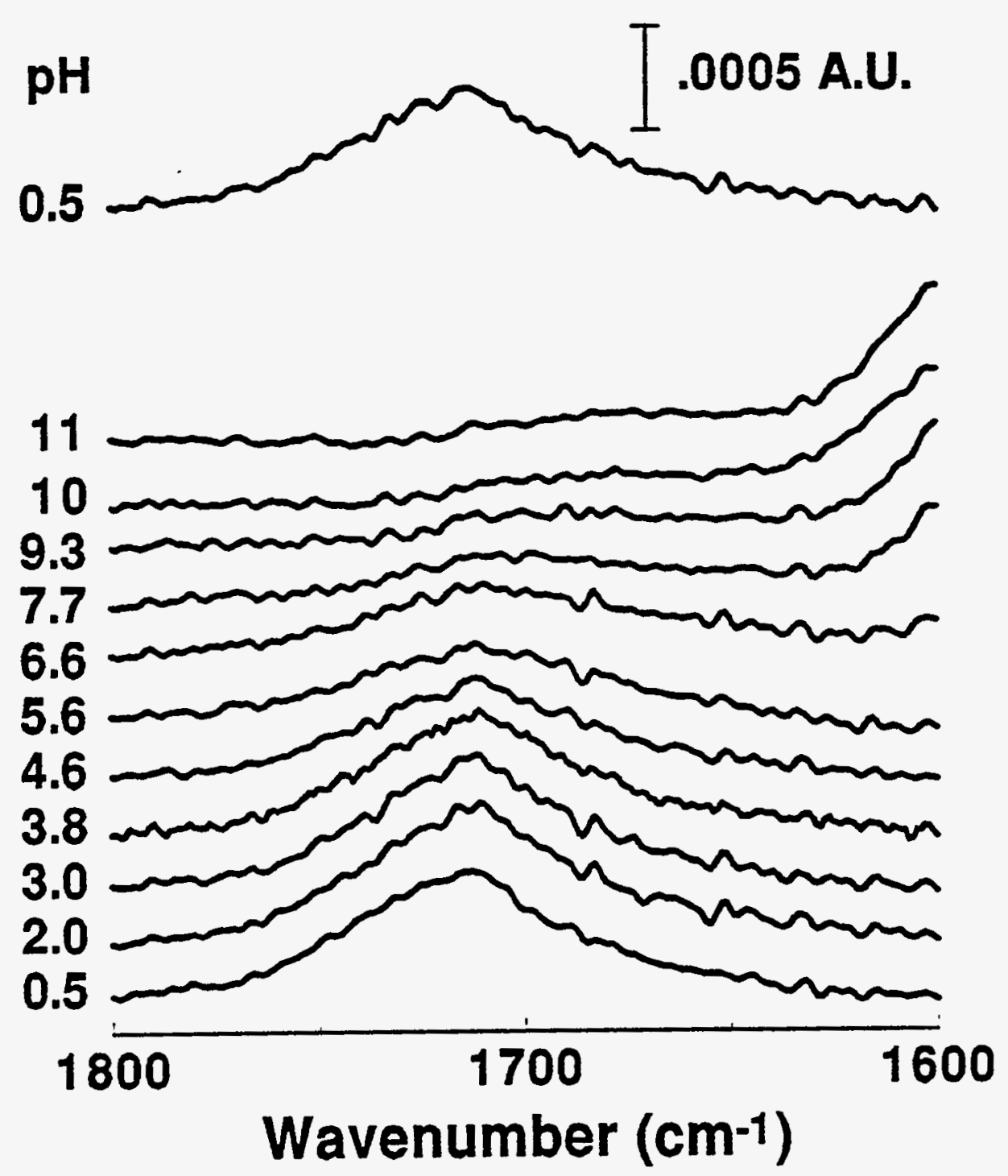

Figure 26. IRRAS spectra in the $\mathrm{C}=\mathrm{O}$ stretching region for a $\mathrm{HO}_{2} \mathrm{C}\left(\mathrm{CH}_{2}\right)_{2} \mathrm{~S} / \mathrm{Au}$ as a function of solution $\mathrm{pH}$. 


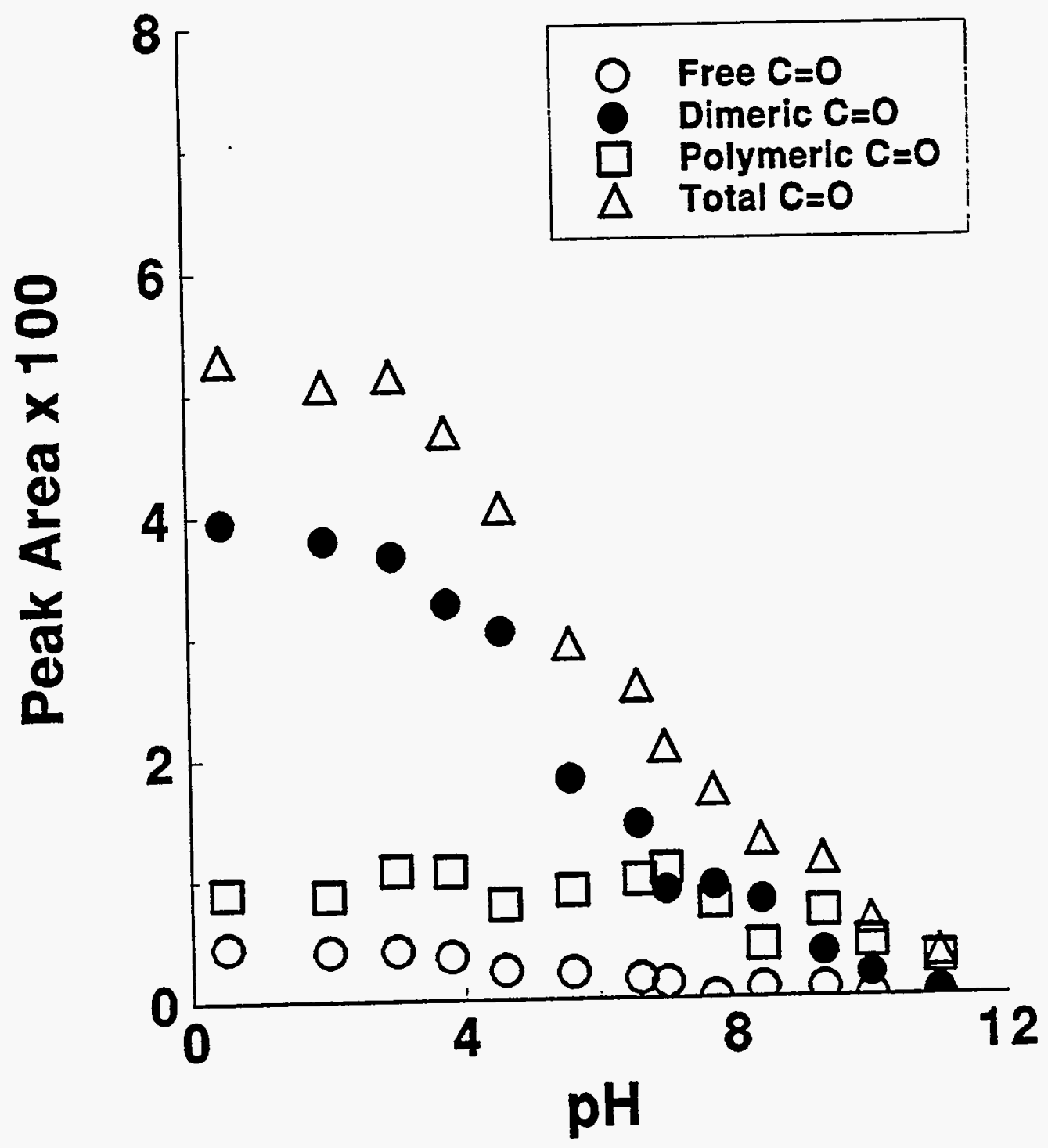

Figure 27. The IRRAS peak area of the $\mathrm{C}=\mathrm{O}$ peaks of the IRRAS spectra for a $\mathrm{HO}_{2} \mathrm{C}\left(\mathrm{CH}_{2}\right)_{2} \mathrm{~S} / \mathrm{Au}$ monolayer as a function of solution $\mathrm{pH}$. 


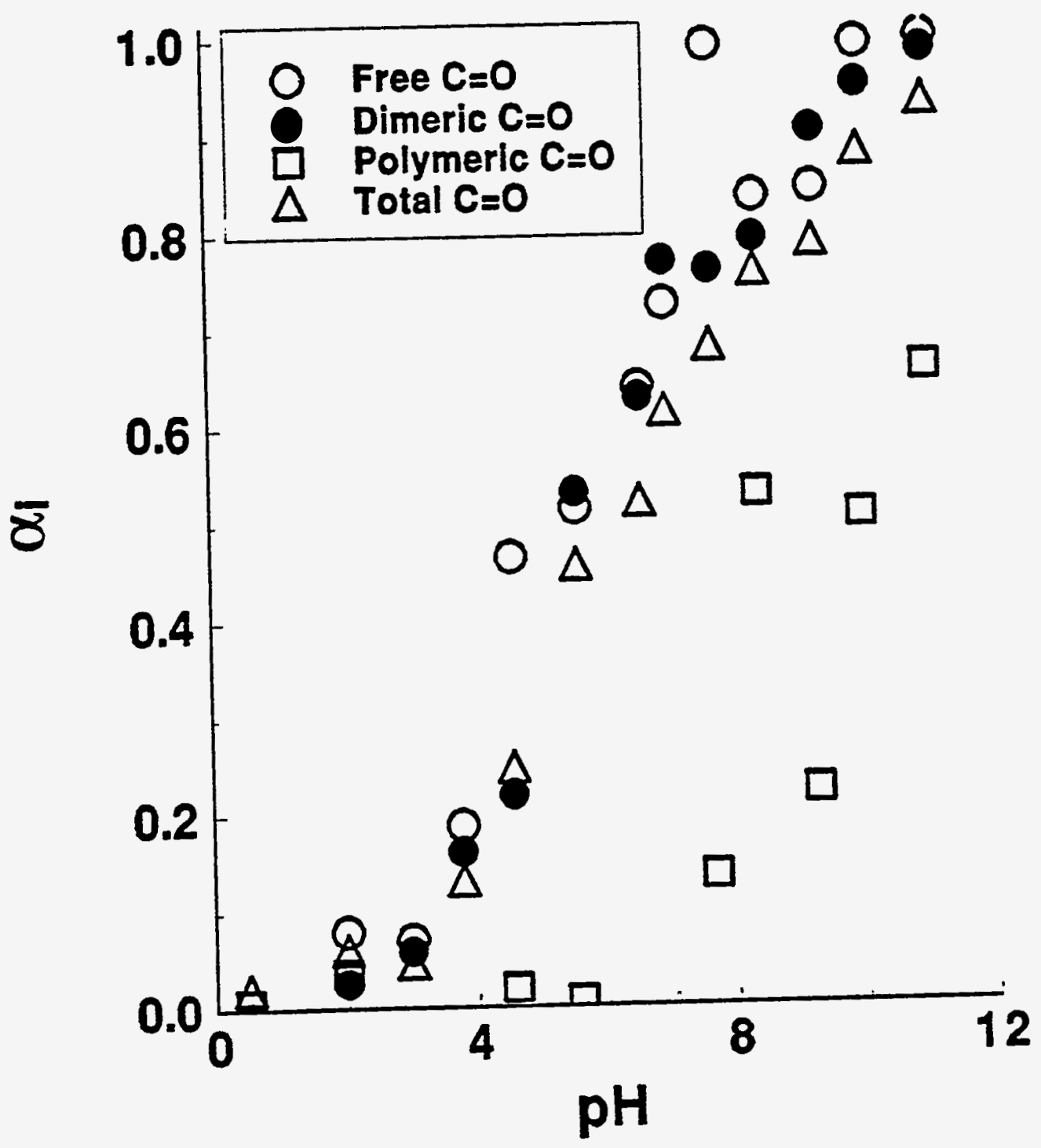

Figure 28. The degree of ionization $\alpha_{\mathrm{i}}$ of $\mathrm{H} \mathrm{HO}_{2} \mathrm{C}\left(\mathrm{CH}_{2}\right)_{2} \mathrm{~S} / \mathrm{Au}$ monolayer as a function of solution $\mathrm{pH}$. 


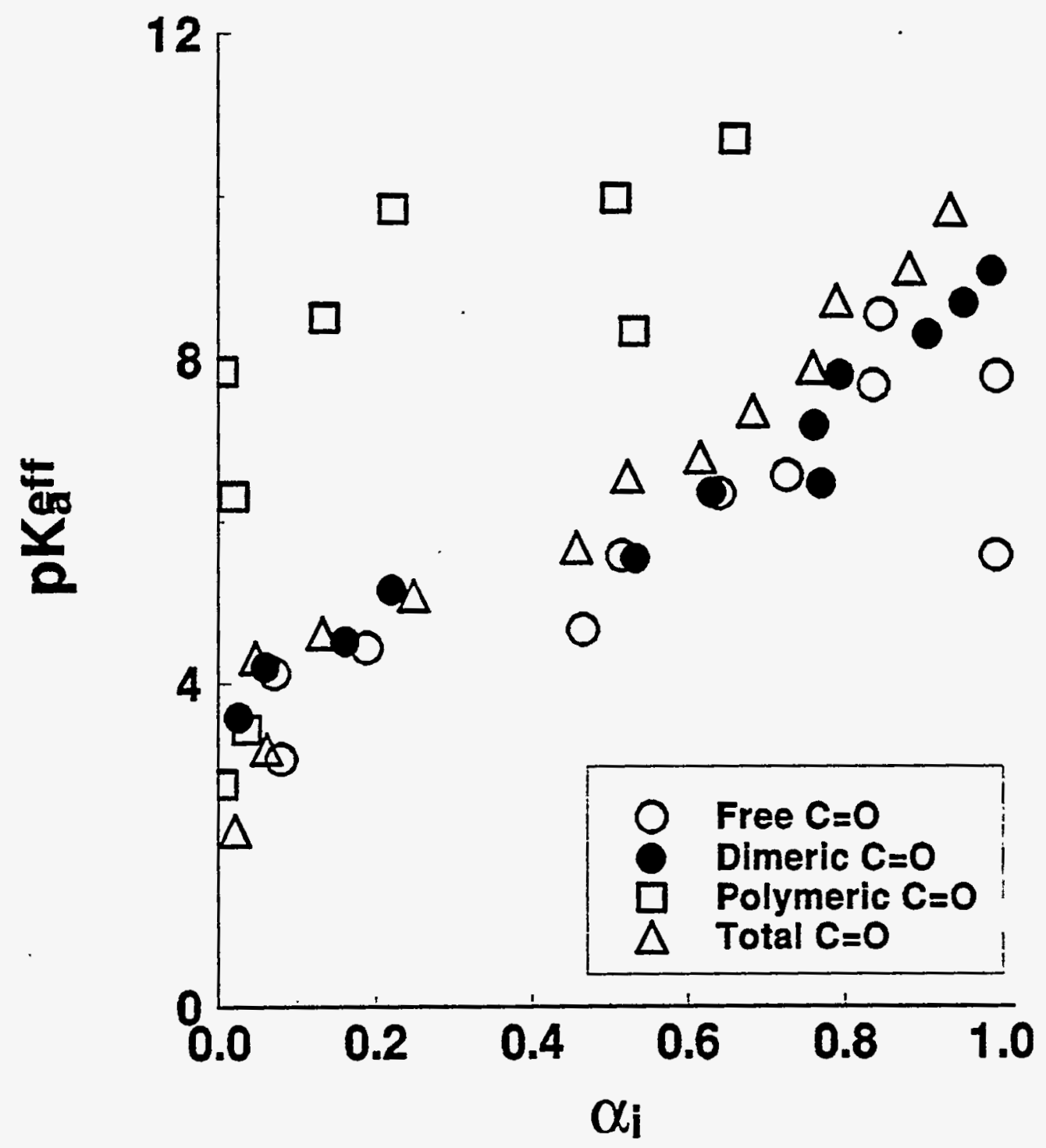

Figure 29. The effective $\mathrm{pK}_{\mathrm{a}}$ of a $\mathrm{HO}_{2} \mathrm{C}\left(\mathrm{CH}_{2}\right)_{2} \mathrm{~S} / \mathrm{Au}$ monolayer as a function of the extent of ionization $\alpha_{i}$. 
of ionization or the effective $\mathrm{pK}_{\mathrm{a}}$. Fortunately, the free $\mathrm{v}(\mathrm{C}=\mathrm{O})$ and the hydrogen-bonded polymeric $v(C=0)$ peaks are relatively small, the hydrogen-bonded dimeric $v(\mathrm{C}=\mathrm{O})$ peak is still can be used to estimate the overall acid property assuming the value of the overall effective $\mathrm{pK}_{\mathrm{a}}$ is virtually the same as that of the dimeric acid. The extent of ionization is very small till $\mathrm{pH} 5$ and starts to increase rapidly with a transition of at least $8 \mathrm{pH}$ units as shown in Figure 35. The $\mathrm{pK}_{\mathrm{a}}$ from IRRAS measurement for the dimeric acid is 6.5.

The value of the effective $\mathrm{pK}_{\mathrm{a}}$ is $\sim 7$ in the beginning of ionization and starts to increase after $40 \%$ of ionization to a value of 11 at $80 \%$ of ionization as shown in Figure 36. The effective $\mathrm{pK}_{2}$ value at $50 \%$ of ionization is 6.6. Since the orientation of this monolayer has changed upon ionization, there is no guarantee that the $v(C=0)$ dipole remains the same. The above analysis will not be valuable unless the $v(C=0)$ dipole remains the same orientation during the titration process. There is no $v\left(\mathrm{COO}^{-}\right)$peak observed for the PUAD/Au monolayer. This does not mean there is no $\mathrm{COO}^{-}$groups at all, but instead the $\mathrm{COO}^{-}$groups is in the monolayer with both the $v_{a}\left(\mathrm{COO}^{-}\right)$and $v_{s}\left(\mathrm{COO}^{-}\right)$dipole parallel to the surface so that there is no IRRAS signal can be seen. 


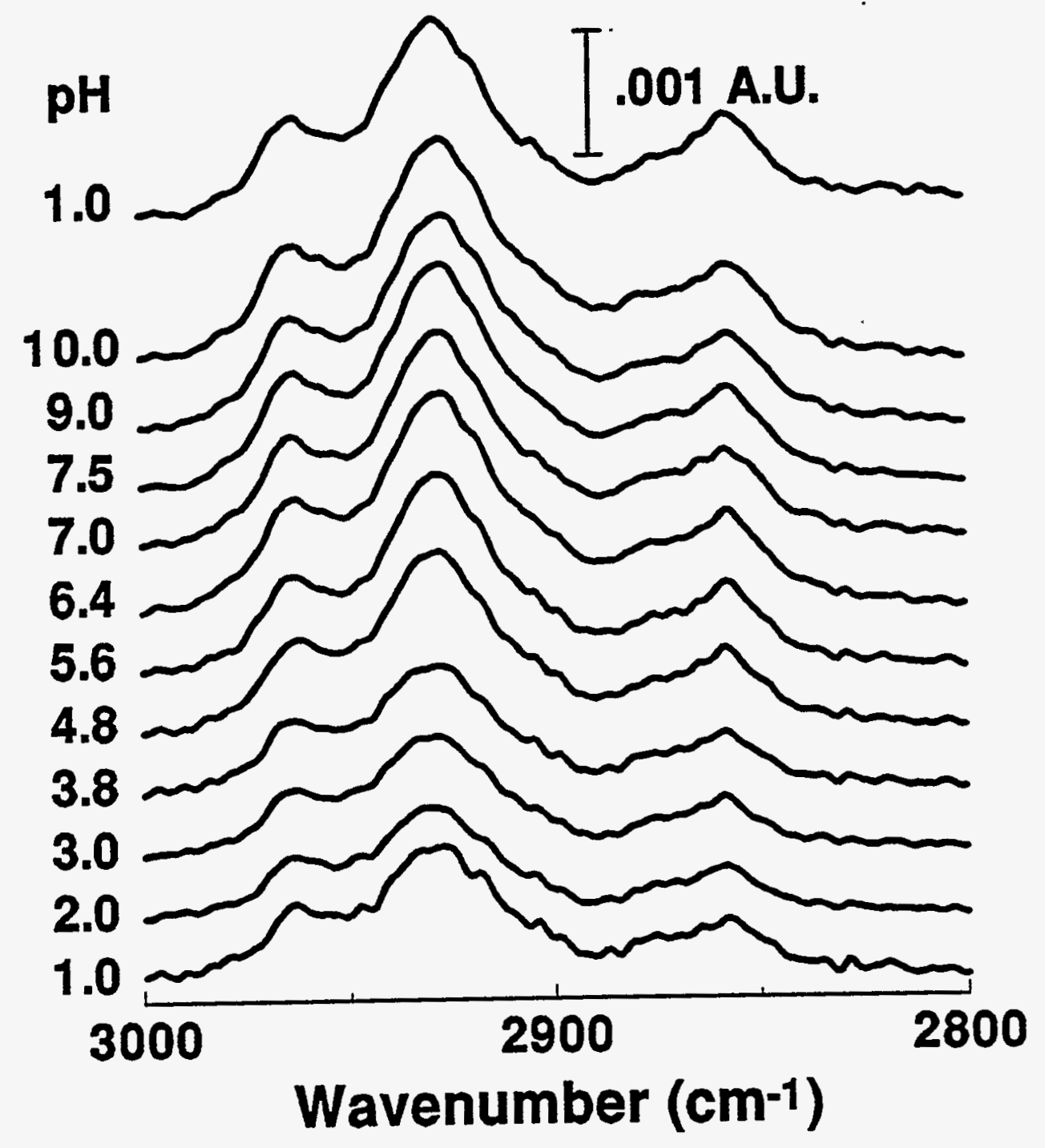

Figure 30. IRRAS spectra in the high energy region for a monolayer of $\mathrm{HO}_{2} \mathrm{C}\left(\mathrm{CH}_{2}\right)_{10} \mathrm{SS}\left(\mathrm{CH}_{2}\right)_{4} \mathrm{CH}_{3}$ at gold as a function of solution $\mathrm{pH}$. 


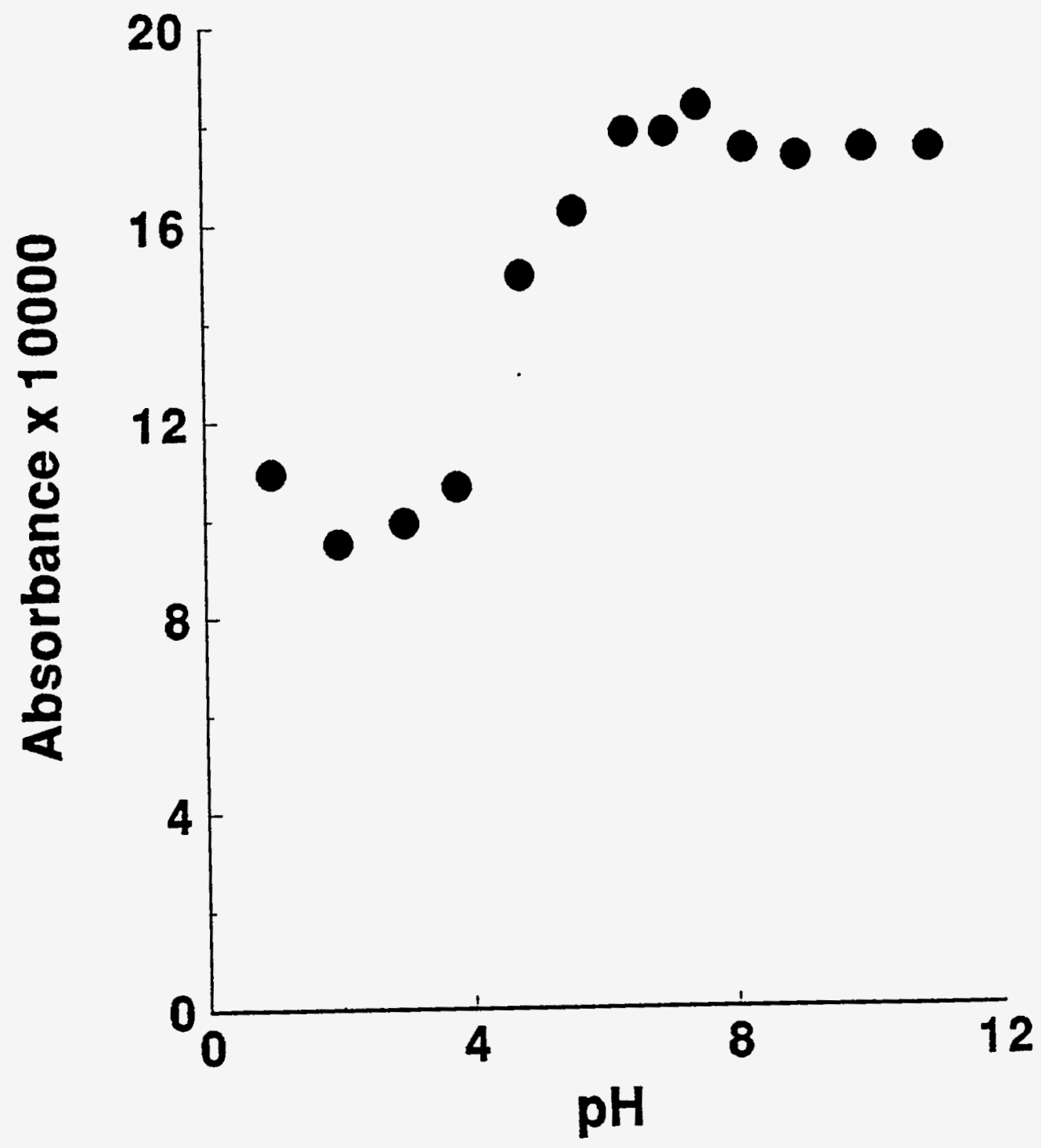

Figure 31. The IRRAS peak intensity at $2926 \mathrm{~cm}^{-1}$ for a monolayer of $\mathrm{HO}_{2} \mathrm{C}\left(\mathrm{CH}_{2}\right)_{10} \mathrm{SS}\left(\mathrm{CH}_{2}\right)_{4} \mathrm{CH}_{3}$ at gold as a function of solution $\mathrm{pH}$. 


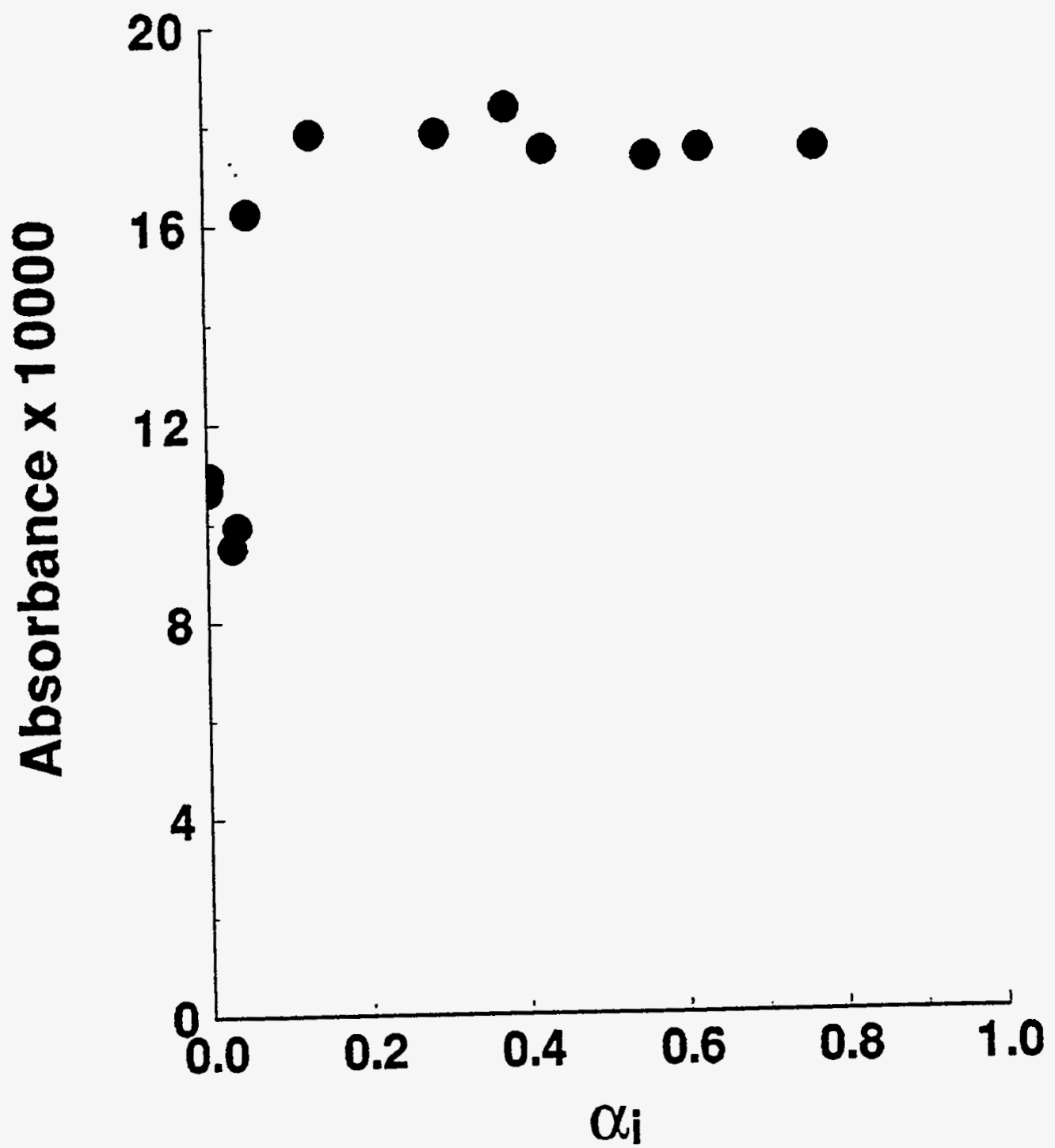

Figure 32. The IRRAS peak intensity at $2926 \mathrm{~cm}^{-1}$ for a monolayer of $\mathrm{HO}_{2} \mathrm{C}\left(\mathrm{CH}_{2}\right)_{10} \mathrm{SS}\left(\mathrm{CH}_{2}\right)_{4} \mathrm{CH}_{3}$ at gold as a function of the extent of ionization $\alpha_{\mathrm{i}}$. 


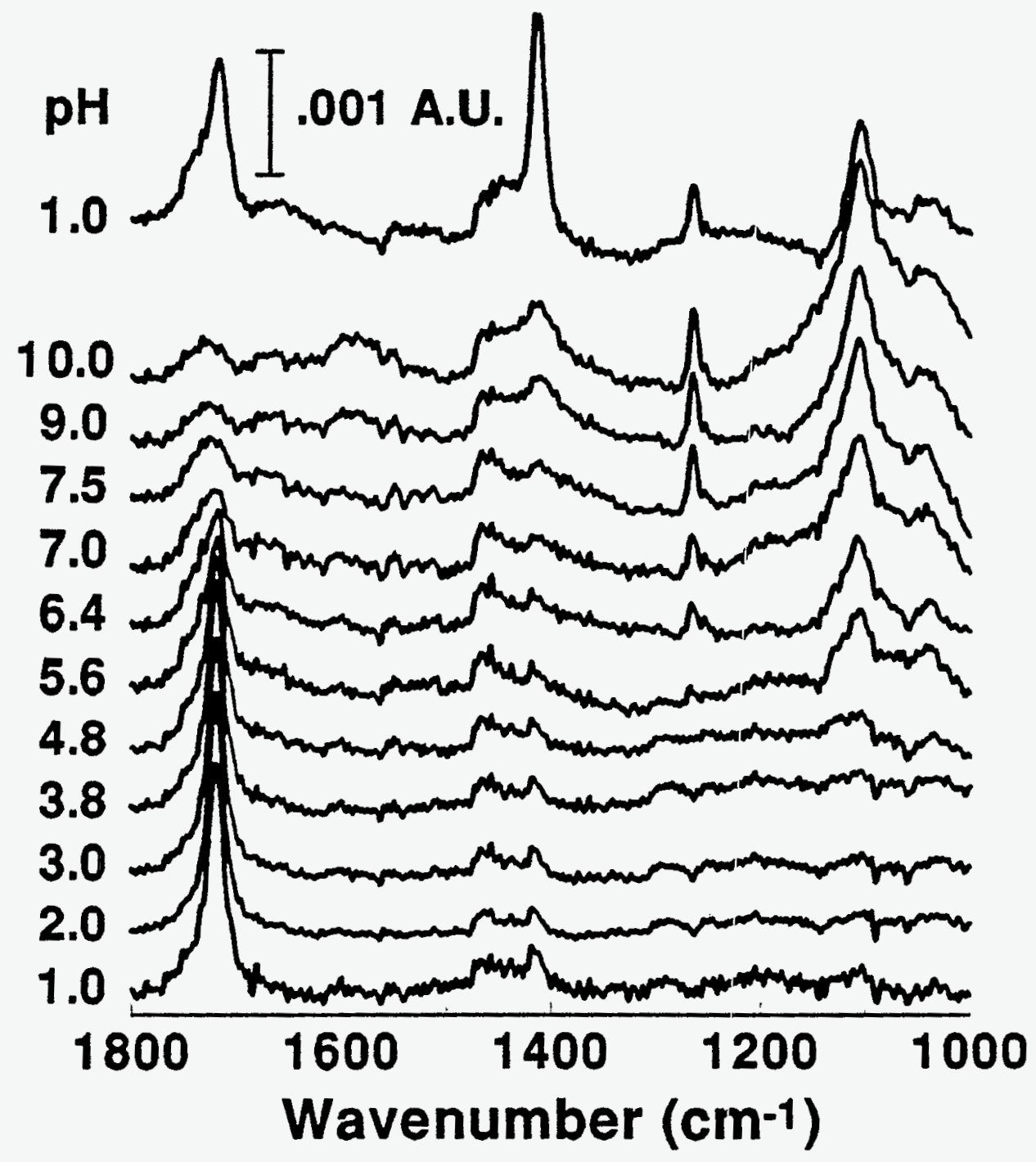

Figure 33. IRRAS spectra in the low energy region for a monolayer of $\mathrm{HO}_{2} \mathrm{C}\left(\mathrm{CH}_{2}\right)_{10} \mathrm{SS}\left(\mathrm{CH}_{2}\right)_{4} \mathrm{CH}_{3}$ at gold as a function of solution $\mathrm{pH}$. 


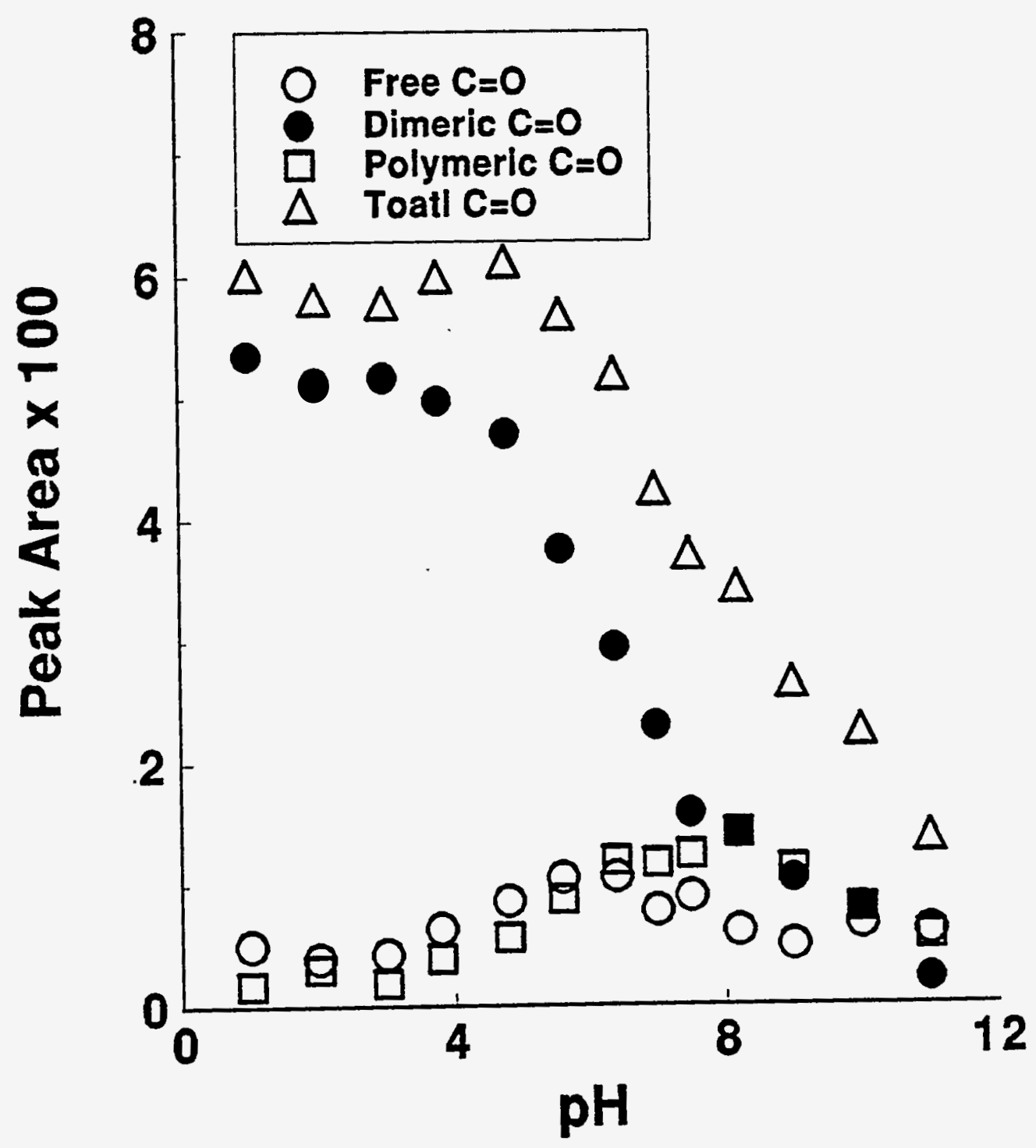

Figure 34. The IRRAS peak area of the $\mathrm{r}(\mathrm{C}=\mathrm{O})$ peaks of a monolayer of $\mathrm{HO}_{2} \mathrm{C}\left(\mathrm{CH}_{2}\right)_{10} \mathrm{SS}\left(\mathrm{CH}_{2}\right)_{4} \mathrm{CH}_{3}$ at gold as a function of solution $\mathrm{pH}$. 


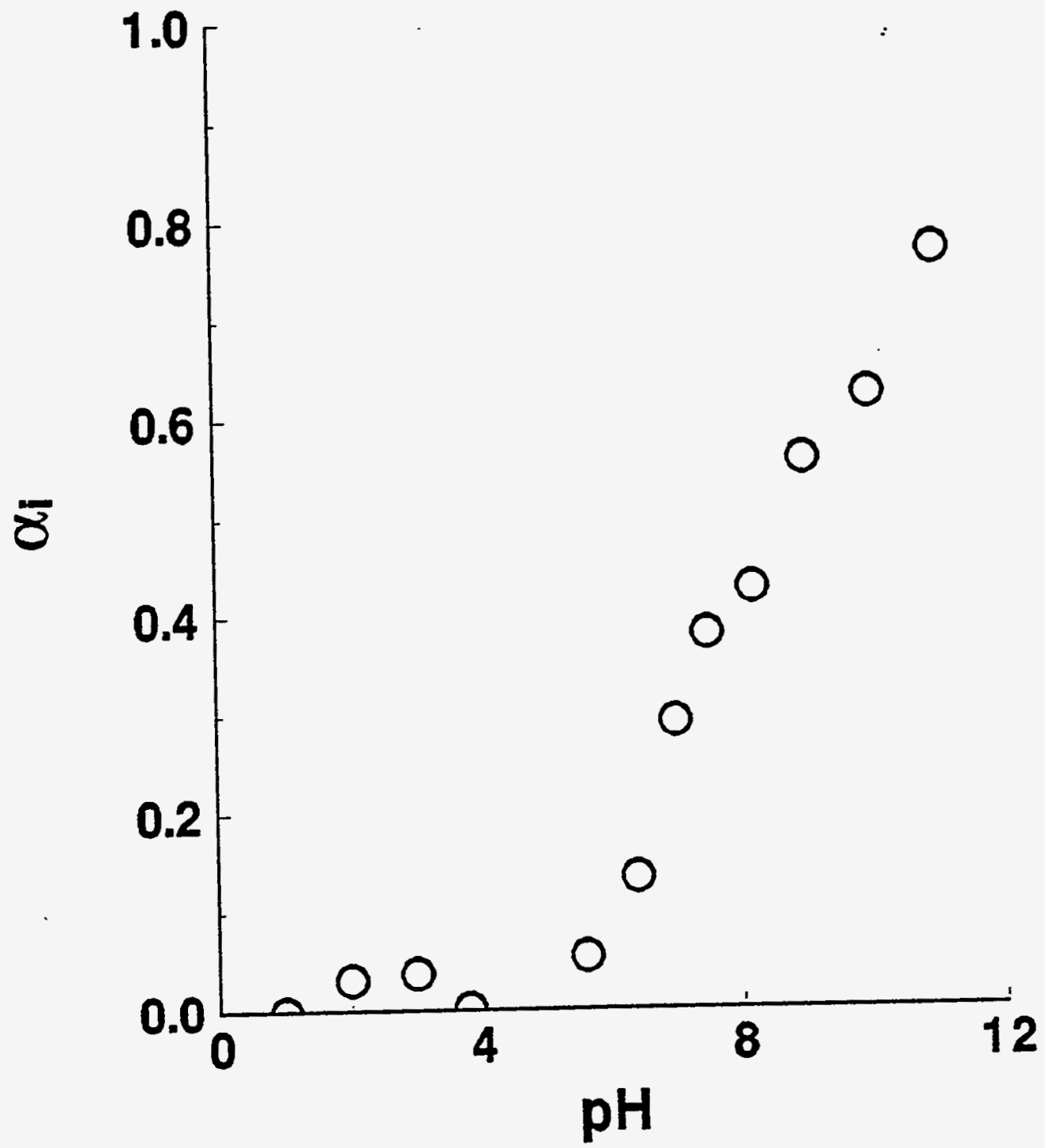

Figure 35. The degree of ionization $\alpha_{\mathrm{i}}$ of a monolayer of $\mathrm{HO}_{2} \mathrm{C}\left(\mathrm{CH}_{2}\right)_{10} \mathrm{SS}\left(\mathrm{CH}_{2}\right)_{4} \mathrm{CH}_{3}$ at gold as a function of solution $\mathrm{pH}$. 


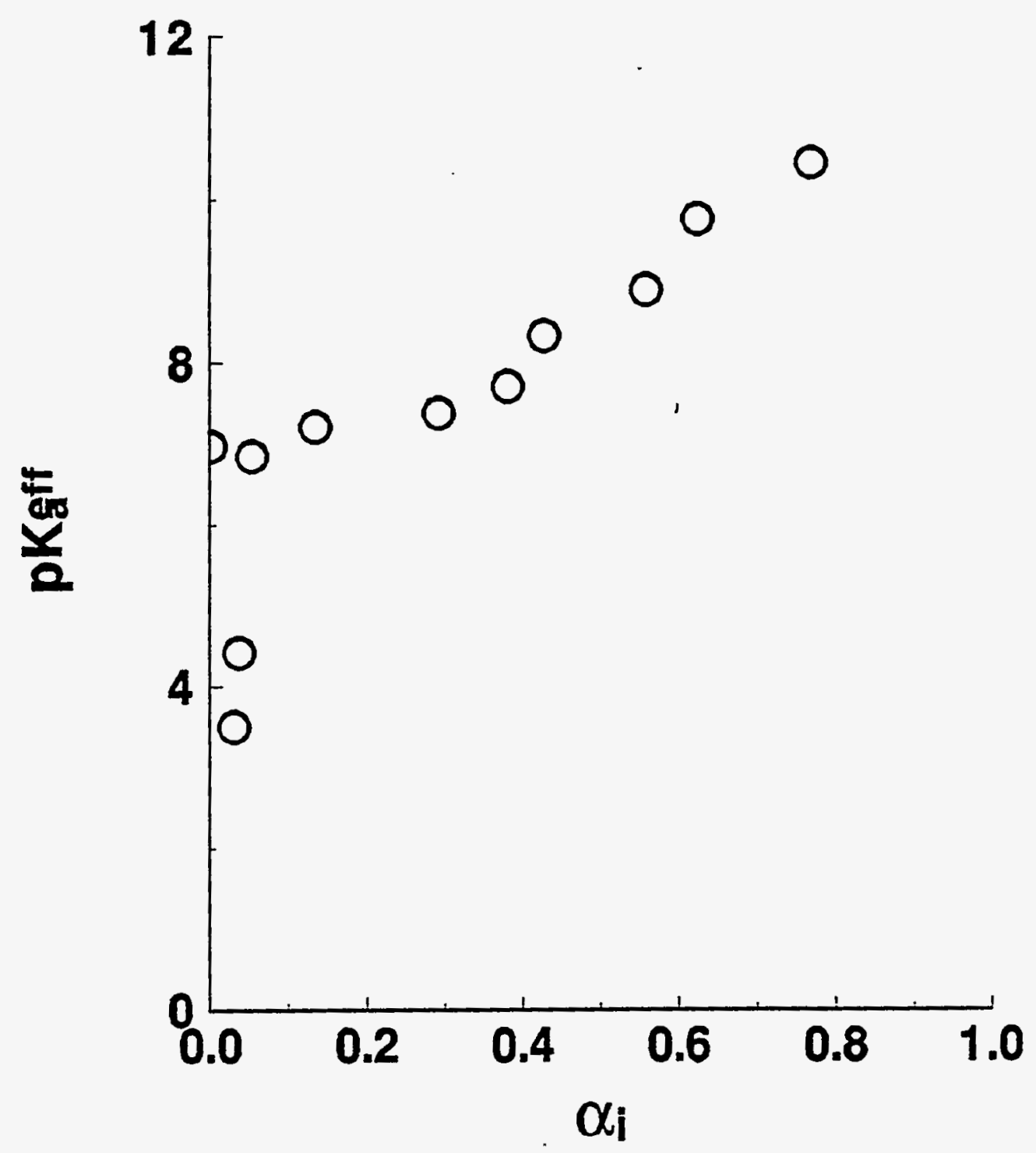

Figure 36. The effective $\mathrm{pK}_{\mathrm{a}}$ of a monolayer of $\mathrm{HO}_{2} \mathrm{C}\left(\mathrm{CH}_{2}\right)_{10} \mathrm{SS}\left(\mathrm{CH}_{2}\right)_{4} \mathrm{CH}_{3}$ at gold as a function of the extent of onization $\alpha_{\mathrm{i}}$. 


\section{CONCLUSIONS}

The spatial structure of densely-packed carboxylic acid-terminated monolayers remains intact, but that of a loosely-packed monolayer is disturbed by the incorporation of the electrolyte and buffer upon ionization. The carboxylate plane of the latter is oriented parallel to surface so that there is no IRRAS signal observed, though monolayers have been ionized.

For all acid studied, the effective $\mathrm{pK}_{\mathrm{z}}$ increases as the extent of ionization $\alpha_{\mathrm{i}}$, and the effective $\mathrm{pK}_{\mathrm{a}}$ values at $50 \%$ of ionization for the free acid, the hydrogen-bonded dimeric acid, and the hydrogen-bonded polymeric acid are around 6,8 , and 10 , respectively. The difference in $\mathrm{pK}_{\mathrm{a}}$ values is mainly due to the difference in the strength of the hydorgen bond.

All three acid groups are weaker acids than the corresponding adsorbate precursor in aqueous solution. The values of the effective $\mathrm{pK}_{2}$ at $50 \%$ of ionization for MHA, MUA, PUAD, and MPA are 7.2, 8.0, 6.5 and 6.0 , respectively. 


\section{REFERENCES}

(1) Bain, C. D.; Whitesides; G. M. Angew. Chem., Int. Ed. Engl. 1989, 28, 506 , and references therein.

(2) Whitesides, G. M.; Laibinis, P. E. Langmuir 1990, 6, 87, and references therein.

(3) Ulman, A. An Introduction to Ultra-Thin Organic Films From LangmuirBlodgett to Self-Assembly; Academic Press: San Diego, 1991.

(4) Widrig, C. A.; Chung, C.; Porter, M. D. J. Electroanal. Chem. 1991, 310, 335.

(5) Bryant, M. A.; Pemberton, J. E. J. Am. Chem. Soc. 1991, 113, 8284.

(6) Bain, C. D.; Biebuyck, H. A.; Whitesides, G. M. Langmuir 1989, 5, 723.

(7) Whitesides, G. M.; Laibinis, F. E. Langmuir, 1990, 6, 87.

(8) Nuzzo, R. G.; Zegarski, B. R.; Dubois, L. H. J. Am. Chem. Soc. 1987, 109, 733.

(9) Fenter, P.; Eisenberger, P.; Li, J.; Camillone, N. III; Bernasek, S.; Scoles, G.; Ramanarayanan, T. A.; Liang, K. S. Langmuir 1991, 7, 2013.

(10) Chidsey, C. E. D.; Liu, G.-Y.; Rowntree, P.; Scoles, G. J. Chem. Phys. 1989, 91, 4421.

(11) Widrig, C. A.; Alves, C. A.; Porter, M. D. J. Am. Chem. Soc. 1991, I13, 2805.

(12) Alves, C. A.; Smith, E. L.; Porter, M. D. J. Am. Chem. Soc. 1992, 114, 1222.

(13) Widrig, C. A.; Chung, C.; Porter, M. D. J. Electroanal. Chem. 1991, $310,335$.

(14) Bain, C. D.; Troughton, E. B.; Tao, Y-T.;Evall, J.;Wihitesides, G. M.; Nuzzo, R. G. J. Am. Chem. Soc. 1989, 111, 321.

(15) Nuzzo, R. G.; Zegarski, B. R.; Dubois, L. H. J. Am. Chem. Soc. 1987, 109, 733.

(16) Dubios, L. H.; Zegarski, B. R.; Nuzzo, R. G. J. Chem. Phys. 1993, 98(1), 678 . 
(17) Hallmark, V. M.; Chiang, S.; Rabolt, J. F.; Swalen, J. D.; Wilson. R. J. Phys. Rev. Lett. 1987, 59, 2879.

(18) Alves, C. A.; Porter, M. D. Langmuir 1993, 9, 3507.

(19) Porter, M. D.; Bright, T. B.; Allara, D. L.;Chidsey, C. E. D. J. Am. Chem. Soc. 1987, 109, 3559.

(20) Tarlvo, M. J.; Bowden, E. F. J. Am. Chem. Soc. 1991, 113, 1847.

(21) Li, T. T.-T.; Weaver, M. J. J. Am. Chem. Soc. 1984,106, 6107.

(22) De Long, H. C.; Donohue, J. J.; Buttry, D. A. Langmuir 1991, 7, 2196.

(23) De Long, H. C.; Buttry, D. A. Langmuir 1990, 6, 1319.

(24) Nuzzo, R. G.; Allara, D. L. J. Am. Chem. Soc. 1983, 105, 4481.

(25) Nuzzo, R. G.; Dubois, L. H.; Allara, D. L. J. Am. Chem. Soc. 1990, $112,558$.

(26) Czanderna, A. W.; King, D. E.; Spaulding, D. J. Vac. Sci. Technol. 1991, A9, 2607.

(27) Pale-Grosdemange, C.; Simmon, E. S.; Prime, K. L.; Whitesides, G. M. J. Am. Chem. Soc. 1991, 113, 12.

(28) Bain, C. D.; Whitesides, G. M. J. Am. Chem. Soc. 1988, 110, 5897.

(29) Popenoe, D. D.; Deinhammer, R. S.; Porter, M. D. Langmuir, 1992, 8, 2521.

(30) Finklea, H. O.; Avery, S.; Lynch, M.; Furtsh, T. Langmuir 1987, 3, 409.

(31) Foley, J. K.; Pons, B. S. Anal. Chem. 1985, 57, 945A.

(32) Pons, S.; Foley, J. K.; Russell, J.; Seversion, M. Mordern Aspects of Electrochemistry Eds. Bochris,.J, O'M.; Conway, B. E.; White, R. E. 1986, 17, 223.

(33) Bewick, A.; Kunimatsu, K.; Pons, B. S.; Russell, J. W. J. Electroanal. Chem. 1984, 160, 47.

(34) Goldern, W. G. Fourier Transform Infrared Spectroscopy, Ed. Ferraro, J. R.; Basile, L. J. Vol. 4; New York: Academic Press, 1985.

(35) Kemp, J. C. Polarized Light and Its Interaction with Modulating Devices, a Methology Review; Hillsboro, OR: HINDS International, Inc. 1987.

(36) Popenoe, D. D.; Stole, S. M.; Porter, M. D. Appl. Spectroscopy 1992, 46,79 . 
(37) Wu, C.-C.; Porter, M. D. unpublished data.

(38) Stole, S. M.; Porter, M. D. Langmuir 1990, 6, 1199.

(39) Hansen, W. N.; Wang, C. L.; Humpherys, T. W. J. Electroanal. Chem. 1978, 90, 137.

(40) Hansen, W. N.; Wang, C. L.; Humpherys, T. W. J. Electroanal. Chem. 1978, 93, 87.

(41) Lolb, D. M.; Hansen, W. N. Surface Sci. 1979, 79, 205.

(42) Hansen, W. N. Surface Sci. 1980, 101, 109.

(43) Fujihira, M.; Kuwana, T. Electrochimica Acta 1975, 20, 565, and references therein.

(44) D'Agostino, A. T.; Hansen, W. N. Surface Sci. 1986, 165, 268.

(45) Sobocinski, R. L.; Bryant, M. A.; Pemberton, J. E. J. Am. Chem. Soc. 1990, 112, 6177 .

(46) Widrig, C. A.; Alves, C. A.; Porter, M. D. J. Am. Chem. Soc. 1991, $113,2805$.

(47) Stole, S. M.; Porter, M. D. Appl. Spectrosc. 1990, 44, 1418.

(48) Walczak, M. M.; Chung, C.; Stole, S. M.; Widrig, C. A.; Porter, M. D. J. Am. Chem. Soc. 1991, 113, 2370.

(49) Wilson, E. B., Jr.; Decius, J. C.; Cross, P. C. Molecular Vibrations; McGraw-Hill: New York, 1955; pp 285-6.

(50) Paper III of this dissertation.

(51) Laibinis, P.; Whitesides, G. M.; Allara, D. L.; Tao, Y.-T.; Parik, A. N.; Nuzzon, R. G. J. Chem. Phys. 1991, 113, 7152.

(52) Allara, D. L.; Nuzzo, R. G. Langmuir 1985, 1, 45.

(53) Allara, D. L.; Nuzzo, R. G. Langmuir 1985, 1, 52.

(54) Scowen, R. V.; Leja, J. Can. J. Chem. 1967, 45, 2829.

(55) Nakamoto, K. Infrared and Raman Spectra of Inorganic and Coordination Compounds; Third ed.; Wiley: New YorK, NY, 1978, 269.

(56) Peter, R. A. Proc. R. Soc. London, Ser. A 1931, 133, 140.

(57) Danielli, J. F. Proc. R. Soc. London, Ser. B. 1937, 122, 155.

(58) Shulman, J. H.; Hughes, A. H. Proc. R. Soc. London, Ser. A. 1932, 138, 430.

(59) Glazer, J.; Dogan, M. Z. Trans. Fraday Soc. 1953, 49, 448. 
(60) Sander, J. V.; Spink, J. A. Nature (London) 1955, 175, 644.

(61) Betts, J. J.; Pethica, B. A. Trans. Fraday Soc. 1956, 52, 1581.

(62) Mille, M. J. Colloid Interface Sci. 1981, 81, 168.

(63) Laskowaski, M.; Scheraga, H. A. J. Am. Chem. Soc. 1954, 76, 6305.

(64) Mille, M.; Van der Kooi, G. J. Colloid Interface Sci. 1977, 61, 475.

(65) Lukac, S. J. Phys. Chem. 1983, 87, 5045.

(66) Holmes-Farley, S. R.; Reamey, R. H.; McCarthy, T. J.; Deutch, J.; Whitesides, G. M. Langmuir 1985, 1, 725. 


\section{PAPER V}

STUDY OF FORMATION AND ESTERIFICATION OF 11MERCAPTOUNDECANOIC ACID MONOLAYERS AT GOLD USING IRRAS AND CONTACT ANGLE MEASUREMENT 


\section{INTRODUCTION}

The self-assembly thiol monolayers on gold has attracted enormous world-wide attention for the last ten years due to the good stability, wellcharacterized systems, easy preparation, and excellent modification properties for applications of these monolayers ${ }^{1-3}$.

The 16-mercaptohexadecanoic acid (MHA) monolayer has been characterized by Nuzzo et al. ${ }^{4}$ They concluded that the structure of a MHA/Au monolayer is almost a perfect zigzag (all trans) conformation. These bands are assigned to the progression of $\mathrm{CH}_{2}$ twisting and wagging modes 5 . From the IRRAS $v\left(\mathrm{CH}_{2}\right)$ modes, the tilt angle and twist angle were calculated to be $32^{\circ}$ and $55^{\circ}$, respectively, with the axis of $\mathrm{C}=\mathrm{O}$ tilted from the surface normal by $66^{\circ} .4$ The thickness of the MHA/Au monolayer was measured with ellisometry method to be $16 \AA^{4}$.

For application purposes, it is very likely to modify a monolayer to construct a new one with the right properties. Therefore, it is essential to understand the surface reaction fully in order to facilitate these modification processes. Although esterification at a polymer surface has been studied by Whitesides et al, the complicated polymer system can not serve as a good model for surface reaction study because of the limited understanding regarding the coverage and the orientation of carboxylic acids at these polymer surface $^{6}$.

In this study, two 11-mercaptoundecanoic acid monolayers on Au with different fractional coverages of 0.50 and 1.00 were used as models for a surface reaction study that probed the relationship between the coverage and extent of reaction using IRRAS and contact angle measurements. 


\section{EXPERIMENTAL SECTION}

\section{Substrate preparation}

Electrodes were prepared by the resistive evaporation of $15-20 \mathrm{~nm}$ of chromium, followed by $200-300 \mathrm{~nm}$ of gold, onto 3 inch by 1 inch glass substrates. The temperature during the evaporation, measured at the plate supporting the substrates, increased to $\sim 50^{\circ} \mathrm{C}$ as a result of radiative heating by the evaporation source. The evaporation rates were 0.1 and $0.5 \mathrm{~nm} / \mathrm{s}$ for chromium and gold, respectively. The pressure in a cryopumped E360A Edwards Coating System during evaporation was $<9 \times 10^{-5} \mathrm{~Pa}$. After $\sim 45 \mathrm{~min}$ cooling time, the evaporator was back-filled with purified $\mathrm{N}_{2}$. The substrates were then removed, analyzed by optical ellipsometry for the determination of the optical functions of the uncoated substrates, and immersed in the thiolcontaining solution.

The surfaces of the uncoated gold electrodes have been characterized previously at both a macroscopic and microscopic level using scanning tunneling microscopy ${ }^{7}$ and electrochemical measurements of surface texture and surface area ${ }^{8}$. Macroscopically, the STM images show that the electrode surface is composed of atomically-terraced crystallites $\sim 25 \mathrm{~nm}$ in diameter. The electrodes have a roughness factor of $\sim 1.3$, which is given as the surface area determined from the oxidative desorption of iodine ${ }^{8}$ divided by the geometric electrode area.

\section{Monolaver preparation}

The MUA monolayers were prepared by their spontaneous adsorption onto the evaporated gold from $0.100 \mathrm{mM}$ solutions in absolute ethanol for 10 sec and 24 hours in order to obtain two film thickness of $8.0 \AA$ and $16.0 \AA$, emersed, rinsed successively with absolute ethanol and hexane, and dried under a stream of prepurified $\mathrm{N}_{2}$.

\section{Esterification}

Samples were left in solution for different lengths of time in $20 \%(\mathrm{v} / \mathrm{v})$ of conc. $\mathrm{HCl}$ ethanol solution. Upon removal from solution, the samples were rinsed with $\mathrm{pH} 1.0 \mathrm{HCl}$ aqueous solution and then placed on a spin-coater and 
spun briefly at $2000 \mathrm{rpm}$ to remove excess solution. The samples were then placed in the $\mathrm{N}_{2}$-purged sample chamber of the IR spectrometer for characterization. Spectra were usually acquired within a few minutes of loading in the chamber, with the rotational vibrational bands of vapor phase water subtracted by using a reference vapor spectrum.

\section{Ellipsometric measurements of film thickness}

The thickness of the monolayers were determined by optical ellipsometry at $632.8 \mathrm{~nm}$ in two steps with a computer-interfaced Gaertner Model L-116B ellipsometer. The angle of incidence was $70^{\circ}$ from the surface normal. Upon removal from the evaporation chamber, the analyzer and polarizer angles for a reflected light beam from each uncoated substrate were measured on at least three different spots. The average complex refractive index for each substrate was then calculated with a two phase parallel layer model from classical electromagnetic theory ${ }^{9}$. After monolayer formation, each sample was again analyzed and the film thickness calculated from a threephase parallel layer model, using the average complex refractive index of the individual sample and a real refractive index of 1.46 for the film. A value of 1.46 , which is representative of the adsorbate precursors ${ }^{10}$, facilitates comparison with thickness data that has been reported for a variety of monolayers ${ }^{2,11-15}$. The influence of several refinements on this treatment of ellipsometric data have been recently discussed ${ }^{11,12,16}$.

\section{Contact angle measurements}

Advancing contact angles $\left(\theta_{\mathrm{a}}\right)$ were measured in air with $\mathrm{pH}=1.0 \mathrm{HCl}$ aqueous solution as probe liquids by using a Rame-Hart Model 100-00 115 goniometer. For these measurements, a $2.0 \mathrm{~mL}$ droplet was formed on the substrate with the needle of the syringe in the droplet. The value of $\theta_{a}$ was determined as the volume of the droplet was slowly increased.

\section{Infrared spectroscopy}

Infrared spectra were acquired with either a Nicolet 740 or a 750 FT-IR spectrometer. Spectra of the adsorbate precursors were obtained by their dispersion in $\mathrm{KBr}$. Spectra of the monolayers were obtained in a single 
reflection mode by using $p$-polarized light incident at $82^{\circ}$ from the surface normal and a liquid nitrogen cooled $\mathrm{HgCdTe}$ detector. A home-built sample holder was used to position reproducibly the substrates in the sample chamber of the spectrometer ${ }^{17}$. The spectrometer was purged with boil-off from liquid $\mathrm{N}_{2}$. Spectra were obtained by referencing 1024 sample scans to 1024 background scans at $2 \mathrm{~cm}^{-1}$ resolution (zero filled) with Happ-Genzel apodization. All spectra are reported as $-\log \left(R / R_{0}\right)$, where $R$ is the reflectance of the coated substrate and $R_{0}$ is that of a reference $A u$ substrate. The reference substrates were prepared by immersion of an uncoated gold substrate into $\sim 1 \mathrm{mM}$ ethanolic solution of octadecanethiol- $\mathrm{d}_{37}$. These films provide a substrate free from detectable IR bands in the regions relevant to these studies as determined when referenced to an uncoated $\mathrm{Au}$ substrate that was cleaned in a X 1:3 $\mathrm{H}_{2} \mathrm{O}_{2}(30 \%): \mathrm{H}_{2} \mathrm{SO}_{4}$ (con.) solution. Further details of the preparation of the reference substrates are given elsewhere ${ }^{18}$. Caution: The $\mathrm{H}_{2} \mathrm{O}_{2} / \mathrm{H}_{2} \mathrm{SO}_{4}$ solution reacts violently with organic compounds and should be handled with extreme care.

The orientation analysis, which compares the observed spectrum to that calculated for an isotropic array of oscillators of comparable packing density, was accomplished by using the frequency-dependent complex optical constants of the adsorbate precursor or a suitable substitute. The optical constants for 11-mercaptoundecanoic acid was determined from a transmission spectrum after its dispersion as a crystalline material in $\mathrm{KBr}$. From the absorbance values, a Kramers-Kronig transformation ${ }^{19}$ yielded the frequency-dependent complex optical constant.

\section{Chemical and reagents}

The 11-mercaptoundecanoic acid $\left(\mathrm{HO}_{2} \mathrm{C}\left(\mathrm{CH}_{2}\right)_{10} \mathrm{SH}\right)$ (MUA) was synthesized in this group and characterized with IR and NMR. Ethyl alcohol was from Quantum Chemical Corp. (II). The rest of chemicals was obtained from Aldrich. All other reagents were used as received. Aqueous solutions were freshly prepared from water purified by passage through a Milli-Q purification system (Millipore Corp., Bedford, MA). 


\section{RESULTS AND DISCUSSION}

\section{Monolayer formation}

\section{Ellipsometric thickness as a function of immersion time}

The thickness of the MUA monolayer on gold (MUA/Au) was measured as a function of immersion time using optical ellipsometry. The results are shown in Figure 1. In 0.1 mM MUA solution, the thickness of MUA/Au increases rapidly at short immersion times, reaching a value of $\sim 6 \AA$ within 0.1 $\mathrm{min}$. The rate of the process slow thereafter with the thickness doubling within $\sim 4 \mathrm{~min}$ and approaching a limiting value of $16.0 \AA$.

This formation process is not a simple one step Langmuir isotherm surface process. Actually, this result is consistent with a two-step formation process proposed by Whitesides et al. ${ }^{20}$ and confirmed by Shimazu et al. ${ }^{21}$ and Grunze et al. ${ }^{22-24}$ The first step is a Langmuir adsorption process, and the second step is believed to be a result of the combination of replacement of the incorporated solvent and adsorbed impurities and lateral diffusion on the surface to reduce defects and enhance packing ${ }^{20}$. This second process has a much slower rate than that of the first process.

\section{Wetting as a function of immersion time}

The wetting property of the surface as a function of immersion time was characterized with contact angle measurements. The probe liquid of a pH 1.0 $\mathrm{HCl}$ aqueous solution was used to keep the acid monolayers in acidic form and the ionic strength the same. The contact angle of water $\left(\theta_{\mathrm{a}}\right)$ and $\cos \theta_{\mathrm{a}}$ as a function of immersion time are shown in Figure 2. The contact angle decreases very dramatically from $47.0^{\circ}$ to $10.0^{\circ}$ in less than $4 \mathrm{~min}$ and decreases only slightly thereafter.

The observation clearly demonstrates that surface properties of the film are determined by the structure of the monolayer. Therefore, it is possible to manipulate the coverage and the structure of a monolayer to control the surface properties of this film by varying immersion time. In this study, two MUA/Au monolayers with different ellipsometric thicknesses of $8.0 \AA$ and $16.0 \AA$ were prepared in $0.1 \mathrm{mM}$ solution for $10 \mathrm{sec}$ and 24 hours, respectively. Since the 


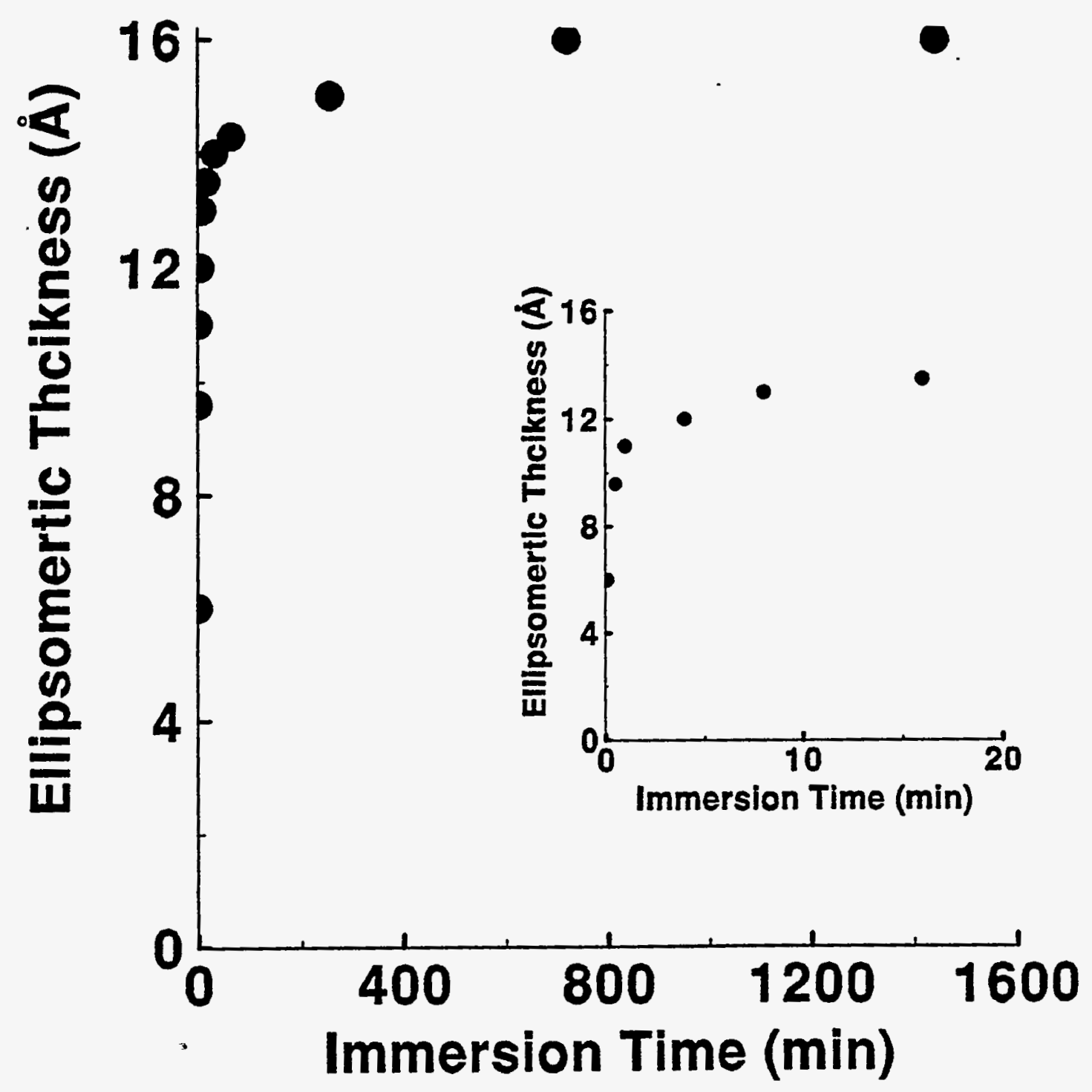

Figure 1. The ellipsometric thickness of a MUA/AU in 0.1mM MUA ethanol solution as a function of immersion time. 


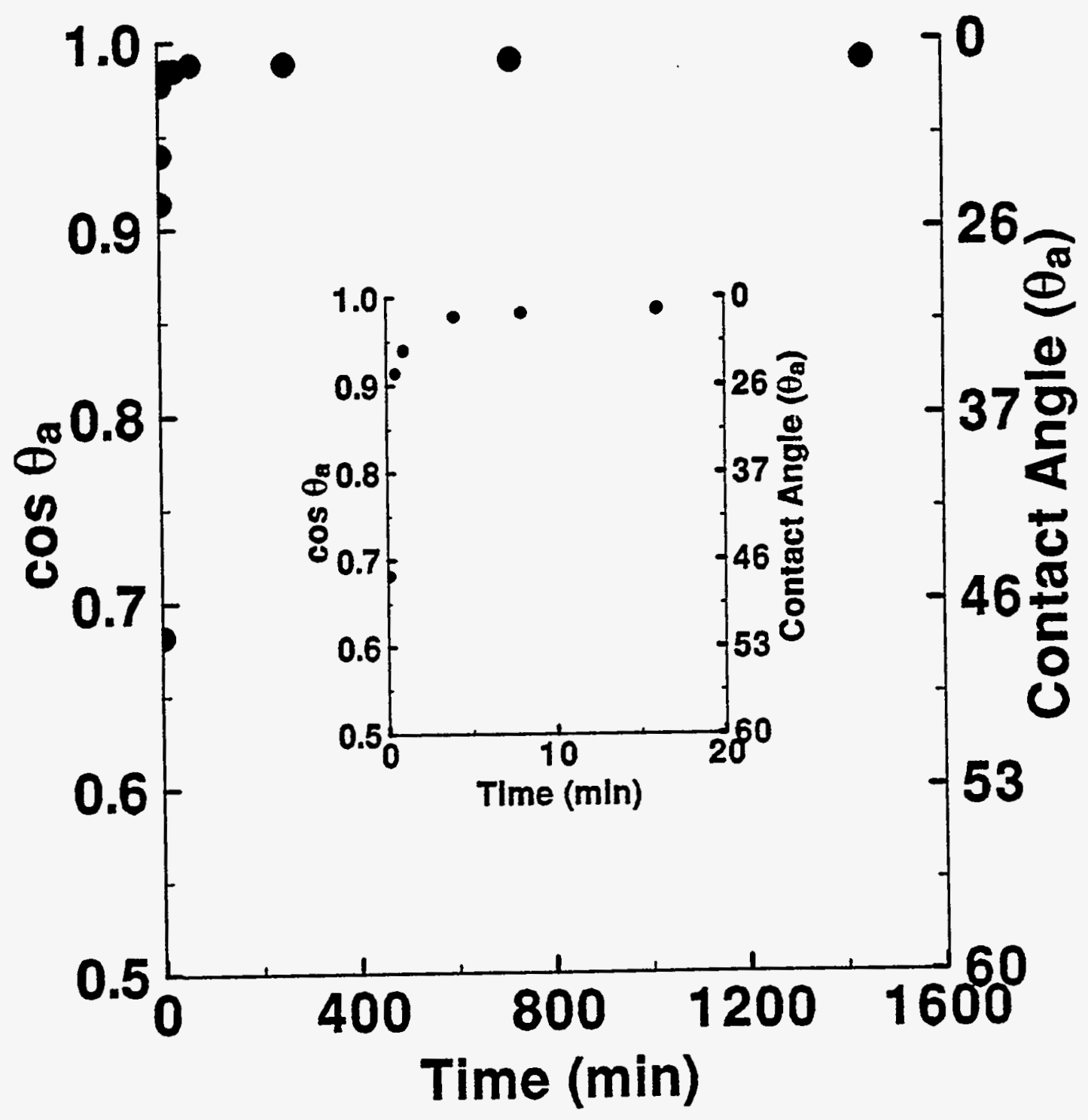

Figure 2. $\cos \theta_{\mathrm{a}}$ of $\mathrm{pH} 1.0 \mathrm{HCl}$ probe solution at a MUA/Au prepared in $0.1 \mathrm{mM}$ MUA ethanol solution as a function of immersion time. 
limiting thickness of the MUA/Au monolayer is $16.0 \AA$, the fractional coverage of the MUA/Au monolayers with the ellipsometric thicknesses of $8.0 \AA$ and $16.0 \AA$ are 0.50 and 1.0 , respectively. The contact angle of water at the halfcoverage MUA/Au monolayer and the full-coverage MUA/Au monolayer are $25.0^{\circ}$ and $8.0^{\circ}$, respectively.

\section{IRRAS spectra as a function of immersion time}

Figure 3 is a series of IRRAS spectra in the high energy region of a MUA/Au monolayer as a function of immersion time. The peak at $2920 \mathrm{~cm}^{-1}$ is assigned to the $v_{\mathrm{a}}\left(\mathrm{CH}_{2}\right)$ mode, and the other peak at $2850 \mathrm{~cm}^{-1}$ is the $v_{\mathrm{s}}\left(\mathrm{CH}_{2}\right)$ mode $\mathrm{m}^{25}$. The shift to low energy of the peak frequencies of both modes with increasing immersion time indicates that the MUA/Au monolayer becomes more crystalline-like ${ }^{25}$ with increasing the coverage. The decrease in the peak area of both modes with increasing immersion time suggests that the average tilt angle of the polymethylene chain decreases with increasing the coverage.

IRRAS spectra in the low region as a function of immersion time is shown in Figure 4. The weak peaks between $1150 \mathrm{~cm}^{-1}$ and $1300 \mathrm{~cm}^{-1}$ are the progression peaks resulting from an all-trans conformation ${ }^{4}$. Peaks between $1400 \mathrm{~cm}^{-1}$ and $1500 \mathrm{~cm}^{-1}$ are due to the $\mathrm{CH}_{2}$ bending mode. In the carbonyl stretching region $\left(1600 \mathrm{~cm}^{-1}-1750 \mathrm{~cm}^{-1}\right)$, the spectrum transfers clearly from a single band at $1717 \mathrm{~cm}^{-1}$ to a doublet. The low energy band at $1717 \mathrm{~cm}^{-1}$, assigned to a hydrogen-bonded dimeric $v(\mathrm{C}=\mathrm{O})$ mode of a carboxylic acid (see below), decreases with increasing immersion time. The band at $1745 \mathrm{~cm}^{-1}$, assigned to a non-hydrogen-bonded $v(\mathrm{C}=\mathrm{O})$ mode of a carboxylic acid, i.e. a free acid, increases with immersion time. In fact, there is another peak at $1680 \mathrm{~cm}^{-1}$ which will be discussed later.

\section{IRRAS characterization}

The average tilt angle of the polymethylene chain

The coverage, the packing density, and the tilt angle can be calculated from the IRRAS data assuming the experimental spectrum has all the peaks corresponding to that in the $\mathrm{KBr}$ spectrum. The experimental and the 


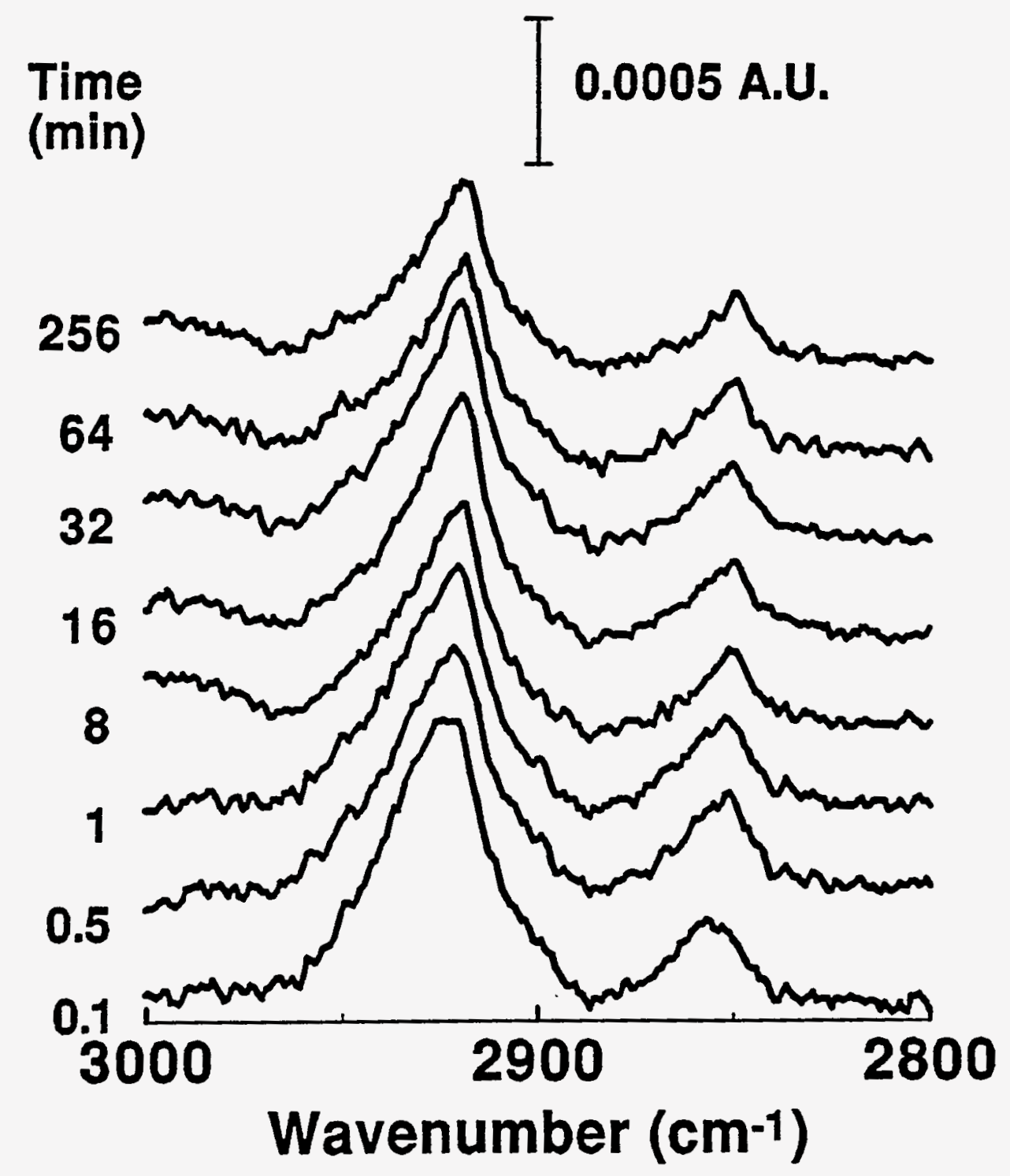

Figure 3. The IRRAS spectra in the high energy region of a MUA/Au prepared in 0.1mM MUA ethanol solution as a function of immersion time. 


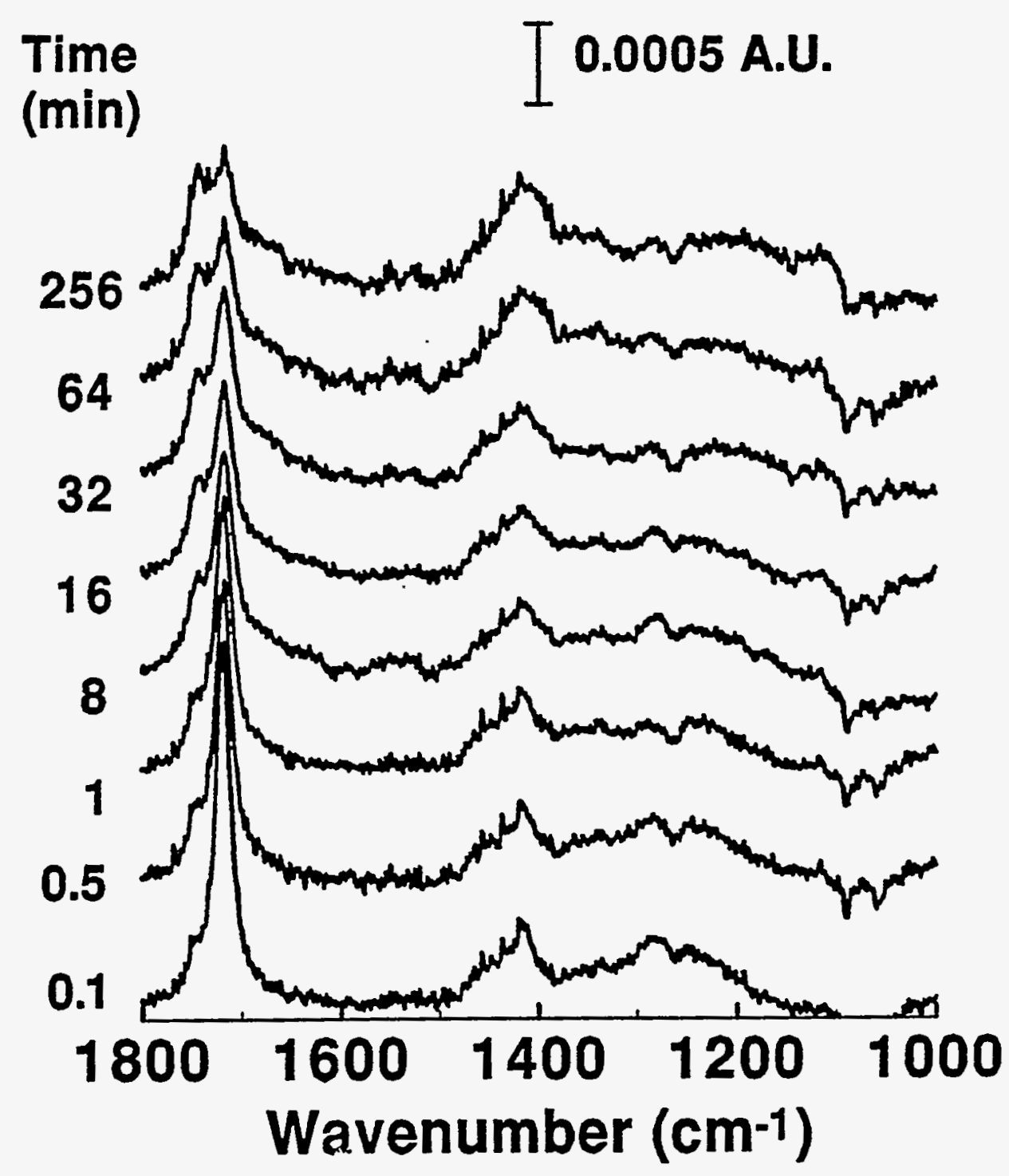

Figure 4. The IRRAS spectra in the low energy region of a MUA/Au prepared in $0.1 \mathrm{mM}$ MUA ethanol solution as a function of immersion time. 
calculated IRRAS spectra of MUAAu obtained as a function of immersion time in the high energy region and the low energy region are shown in Figures 5 and 6, respectively. The vibrational mode assignments are summarized in Table 1. The $v(C=O)$ peak at $1713 \mathrm{~cm}^{-1}$ in $\mathrm{KBr}$ spectrum is a hydrogenbonded dimeric $v(C=0)$ mode which is transformed to three different $v(C=0)$ peaks when MUA is on the Au surface as shown in Figures 6 and 7.

The average tilt angle of the axis of this monolayer can be calculated from the following equation

$$
\cos \theta_{m z}=\left(A_{o b s} / 3 A_{c a l c}\right)
$$

where $A_{o b s}$ and $A_{c a l c}$ are the observed and the calculated peak area, $\theta_{m z}$ is the average tilt angle between the transition dipole moments $(m)$ and the surface normal $(z)^{13}$.

The calculated average tilt angle of the polymethylene chain of the MUA/Au monolayer as a function of immersion time is shown in Figure 8. At the early stage of the adsorption process, the tilt angle of the polymethylene chain is large, suggesting a low packing density. This is consistent with the results from the ellipsometric thickness measurement data. The tilt angle decreases from $57^{\circ}$ to $25^{\circ}$ in the first $4 \mathrm{~min}$ and there is not much decrease thereafter. After $1440 \mathrm{~min}$ of immersion time, this tilt angle of the polymethylene chain of the MUA/Au is $24^{\circ}$ which is less than the value of $32^{\circ}$ determined by Nuzzo et al. for the MHA/Au monolayer ${ }^{4}$. The less tilt angle for short chain monolayers is a result of the less chain-chain interaction for these monolayers than long chain monolayers.

At this tilt angle, this monolayer should have a packing density of $8.0 \mathrm{x}$ $10^{-10} \mathrm{~mol} / \mathrm{cm}^{2}$, i.e. the distance between the nearest neighbor is $5.0 \AA$ which is the same as that for $\mathrm{n}$-alkane thiolate monolayers on $\mathrm{Au}^{7,8}$.

The average tilt angle of carbonyl dipole

The experimental IRRAS spectrum has all the peaks corresponding to the calculated spectrum. Interestingly, the experimental spectrum has two extra peaks: one at $1743 \mathrm{~cm}^{-1}$ which is the non-hydrogen-bonded $v(\mathrm{C}=\mathrm{O})$ 


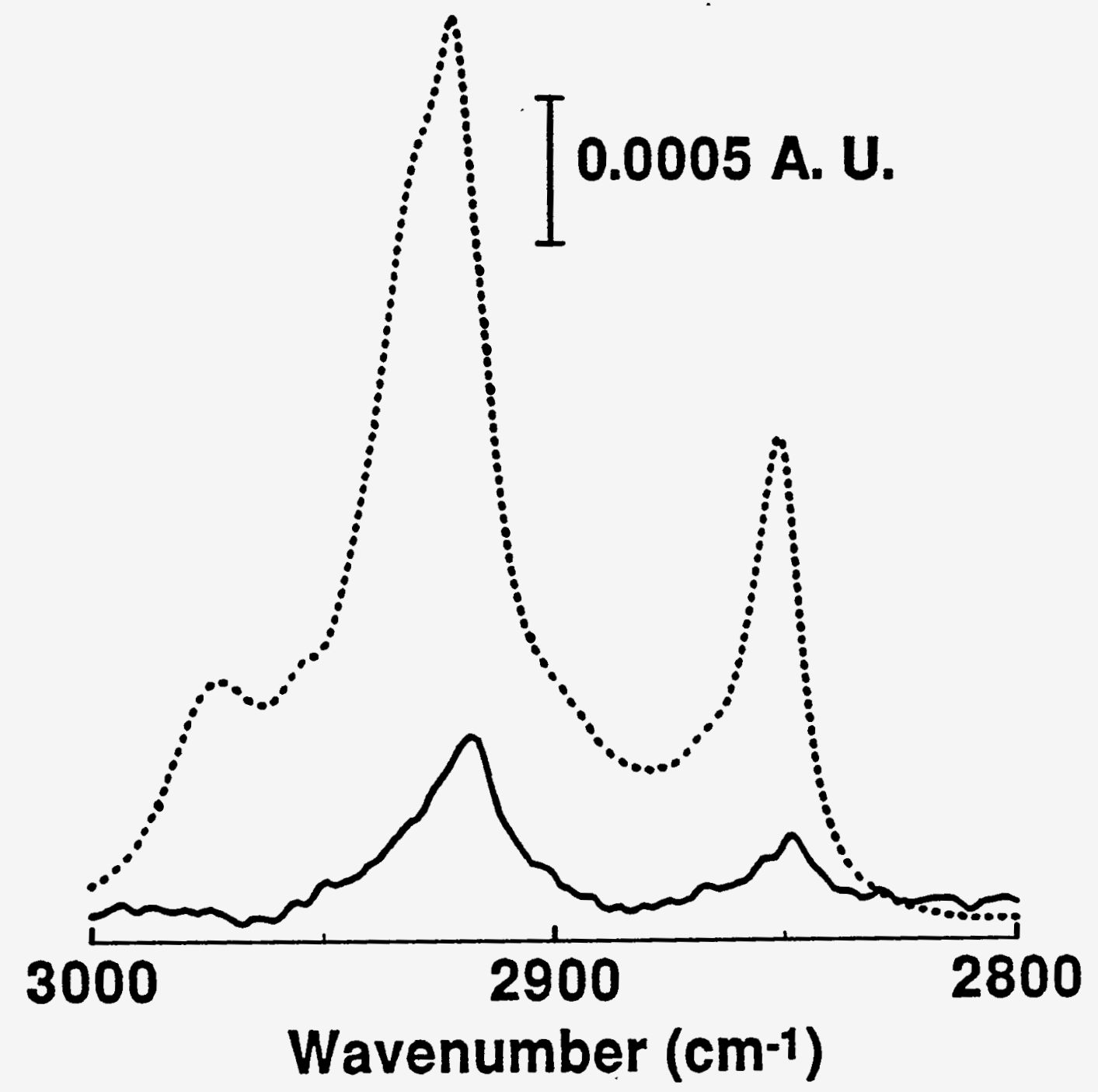

Figure 5. The experimental (solid line) and calculated (dashed line) IRRAS spectra in the high energy region for a full-coverage MUA/Au monolayer. 


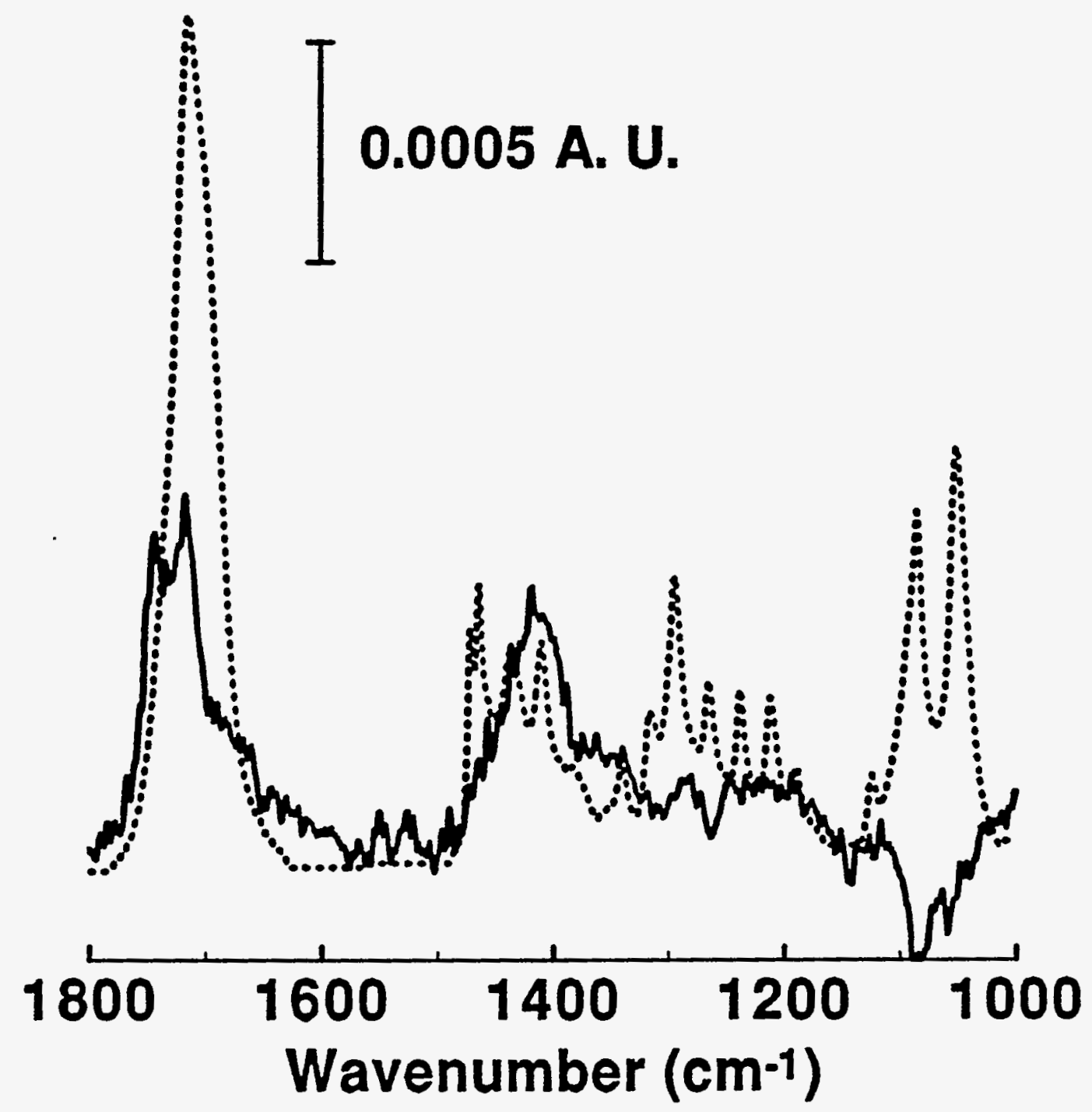

Figure 6. The experimental (solid line) and calculated (dashed line) IRRAS spectra in the low energy region for a full-coverage MUA/Au monolayer. 
Table 1. Peak positions, mode assignments, and transition dipole moment directions of carboxylic acid- and ester-terminated alkanethiolate monolayers at gold.

\section{$\mathrm{HO}_{2} \mathrm{C}\left(\mathrm{CH}_{2}\right)_{10} \mathrm{~S} / \mathrm{Au} \quad \mathrm{C}_{2} \mathrm{H}_{5} \mathrm{O}_{2} \mathrm{C}\left(\mathrm{CH}_{2}\right)_{10} \mathrm{~S} / \mathrm{Au}$}

Mode

direction of

Assignment obs. calc. obs. calc. transition dipole ${ }^{\mathrm{a}}$

\begin{tabular}{|c|c|c|c|c|c|}
\hline \multirow{2}{*}{$\begin{array}{l}v_{\mathrm{a}}\left(\mathrm{CH}_{3, \mathrm{ip}}\right) \\
v_{\mathrm{a}}\left(\mathrm{CH}_{3, \mathrm{op}}\right)\end{array}$} & & \multicolumn{3}{|c|}{$2964^{w}$} & \multirow{2}{*}{$\begin{array}{l}\perp \text { to } \mathrm{C}-\mathrm{CH}_{3} \text { bond in } \\
\mathrm{C}-\mathrm{C}-\mathrm{C} \text { chain plane } \\
\perp \text { to } \mathrm{C}-\mathrm{CH}_{3} \text { bond } \perp \\
\mathrm{C}-\mathrm{C}-\mathrm{C} \text { chain plane }\end{array}$} \\
\hline & & & b & 2954 & \\
\hline$v_{\mathrm{s}}\left(\mathrm{CH}_{3}, \mathrm{FR}_{1}\right)^{\mathrm{c}}$ & & & 2936 & & $\|$ to $\mathrm{C}-\mathrm{CH}_{3}$ bond \\
\hline$v_{a}\left(\mathrm{CH}_{2}\right)$ & 2918 & 2921 & 2918 & 2920 & $\begin{array}{l}\perp \text { to } C-C-C \text { chain } \\
\text { plane }\end{array}$ \\
\hline$v_{\mathrm{s}}\left(\mathrm{CH}_{3}, \mathrm{FR}_{2}\right)^{\mathrm{c}}$ & & & $2878^{w}$ & & $\|$ to $\mathrm{C}-\mathrm{CH}_{3}$ bond \\
\hline$v_{\mathrm{s}}\left(\mathrm{CH}_{2}\right)$ & 2850 & 2851 & 2850 & 2851 & $\begin{array}{l}\| \text { to } \mathrm{C}-\mathrm{C}-\mathrm{C} \text { plane } \\
\text { bisceting } \mathrm{H}-\mathrm{C}-\mathrm{H}\end{array}$ \\
\hline$v(C=0)$ & 1743 & & 1743 & 1740 & \|l to $\mathrm{C}=\mathrm{O}$ bond \\
\hline & $\begin{array}{l}1717 \\
1680\end{array}$ & 1713 & & & \\
\hline$\delta\left(\mathrm{CH}_{2}\right)$ & w & 1471 & 1465 & 1467 & $\begin{array}{l}\text { in } \mathrm{HCH} \text { plane, } \\
\text { bisecting } \mathrm{HCH}\end{array}$ \\
\hline$r(C-0)+$ & & 1437 & & & \\
\hline$\delta(\mathrm{C}-\mathrm{O}-\mathrm{H})^{\mathrm{d}}$ & & 1441 & & 1438 & \\
\hline$\delta\left(\mathrm{a}-\mathrm{CH}_{2}\right)$ & & 1410 & & & \\
\hline$\delta\left(\mathrm{CH}_{3}\right)$ & & & 1382 & 1372 & $\|$ to $\mathrm{C}-\mathrm{CH}_{3}$ bond \\
\hline $\begin{array}{l}\mathrm{CH}_{2} \text { wags } \\
\text { and twists }\end{array}$ & $1200-1350$ & & $1200-135$ & & $\|$ to axis of chain \\
\hline (C-O) & e & 1086 & 1208 & 1212 & \\
\hline (C-O) & e & 1051 & 1179 & 1176 & \\
\hline
\end{tabular}


Table 1. (continued)

aOrientation of transition dipoles for $\mathrm{CH}$ modes assuming all-trans conformation (R. G. Snyder, J. Chem. Phys. 1965, 42, 1744). 'bThe $v_{\mathrm{a}}\left(\mathrm{CH}_{3, \mathrm{op}}\right)$ mode was not observed in the monolayer spectrum. 'The fermiresonance couplet, $v_{\mathrm{s}}\left(\mathrm{CH}_{3}, \mathrm{FR}_{1}\right)$ and $v_{\mathrm{s}}\left(\mathrm{CH}_{3}, \mathrm{FR}_{2}\right)$, is designated by the subscripts 1 and 2, which refer to the higher and lower energy components, respectively. ${ }^{d}$ This band involves both the stretching of the $\mathrm{C}-\mathrm{O}$ bond and the deformation of the C-O-H angle (D. Hadzi and M. Pintar, Spectrochim. Acta, 1958, 12, 162). ${ }^{\circ}$ The peak is canceled by the reference peaks. "Peak intensity is weak. 


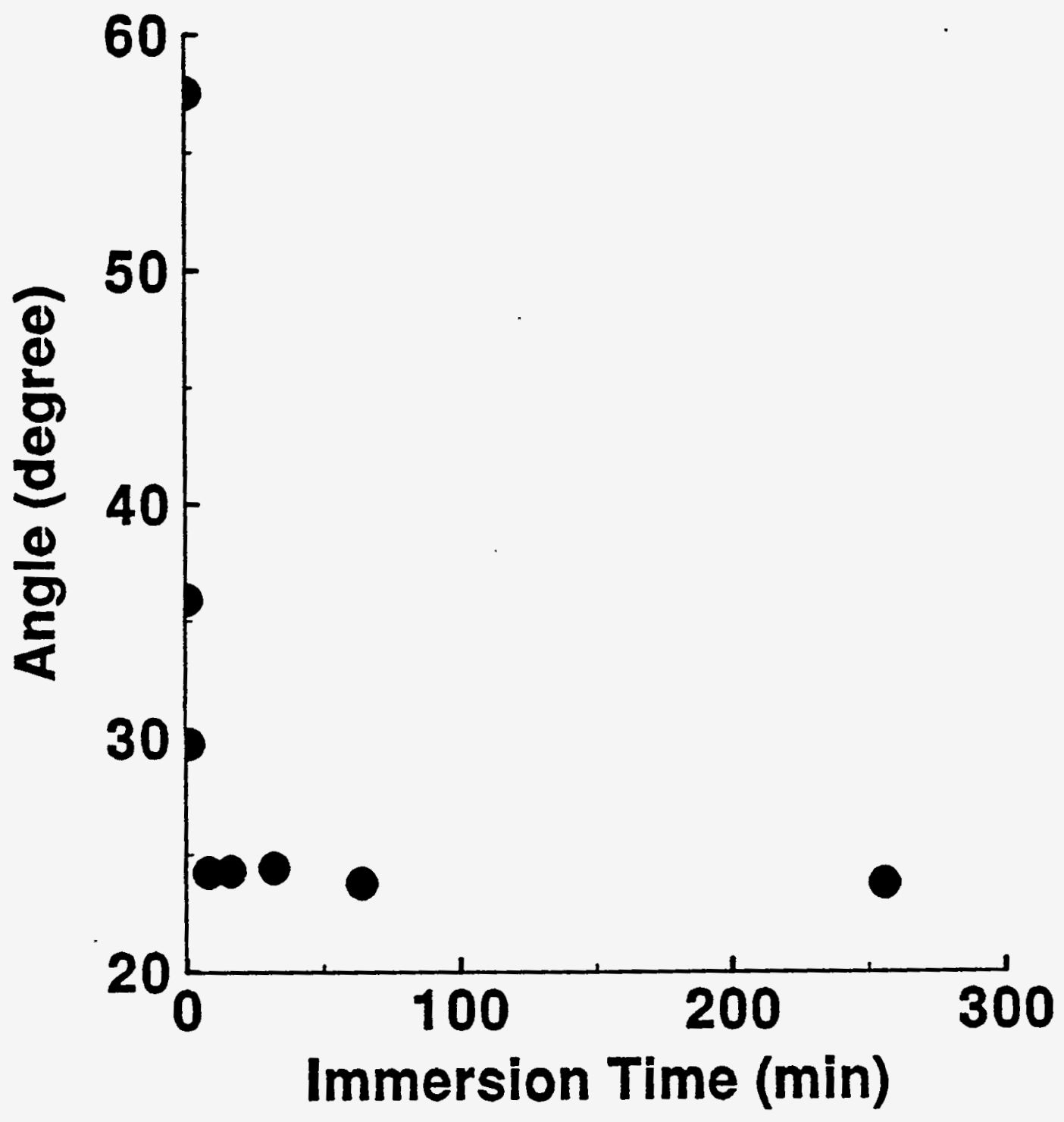

Figure 7. The experimental $v(\mathrm{C}=0)$ mode and deconvoluted $v(\mathrm{C}=0)$ modes for a half-coverage MUA/Au in $20 \%(\mathrm{v} / \mathrm{v}) \mathrm{HCl}$ ethanol solution for $48 \mathrm{~min}$. 

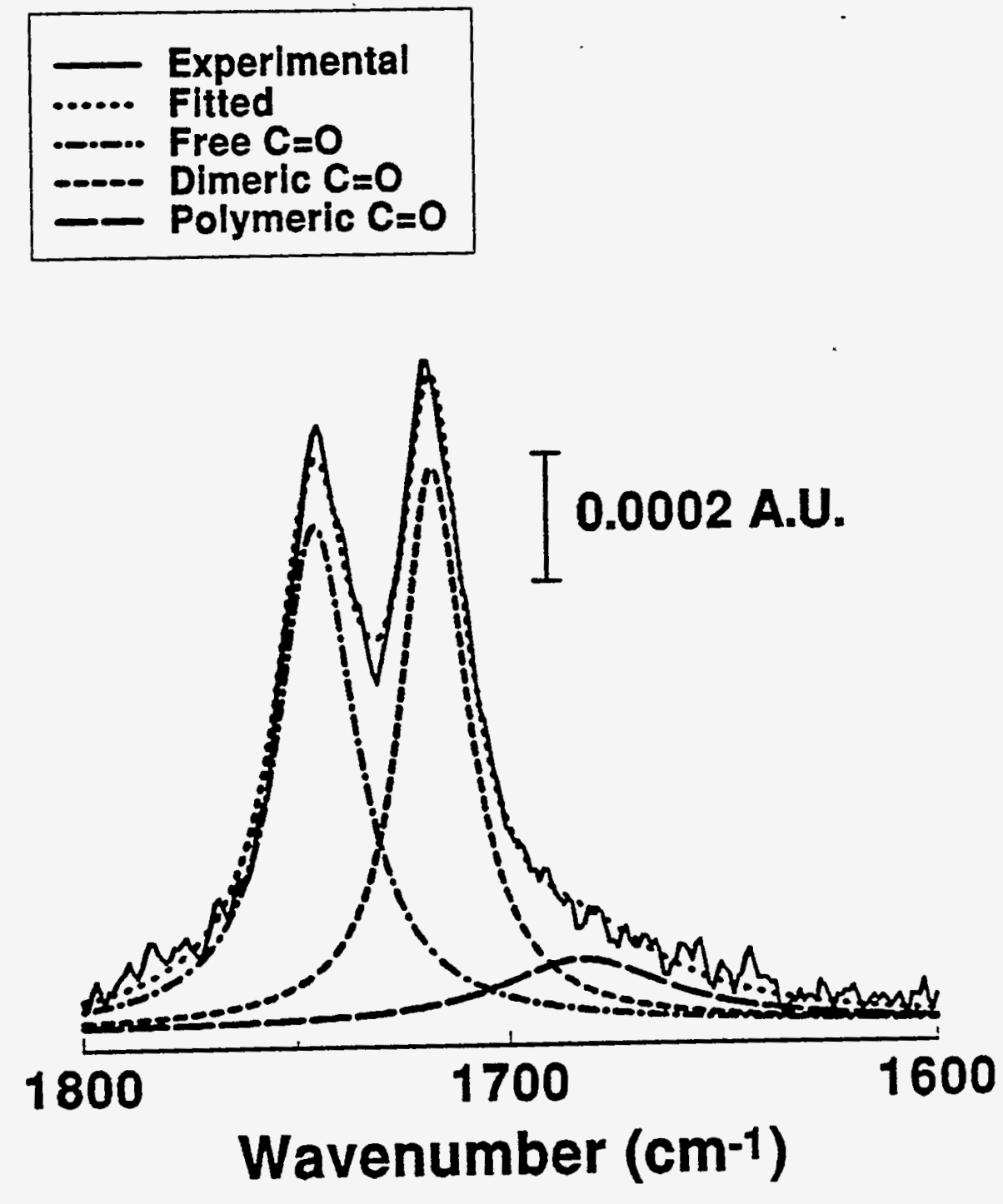

Figure 8. The calculated average tilt angle of a MUA/Au monolayer as a function of immersion time in $20 \%(\mathrm{v} / \mathrm{v}) \mathrm{HCl}$ ethanol solution. 
mode and the other one at $1680 \mathrm{~cm}^{-1}$.

The $v(C=0)$ peaks as a function of immersion time are shown in Figure 4. As one can see, the peak intensity of the hydrogen-bonded dimeric $v(C=0)$ peak decreases with increasing immersion time, while that of the nonhydrogen-bonded $v(\mathrm{C}=\mathrm{O})$ increases with time. The average tilt angle for the $v(\mathrm{C}=0)$ dipole was calculated using equation 1 with a calculated IRRAS spectrum.

The average tilt angle of the $v(C=0)$ dipole starts at $52^{\circ}$ at $0.10 \mathrm{~min}$. and increase rapidly up to $60^{\circ}$ in $4 \mathrm{~min}$. There is only little increase in the tilt angle thereafter.

\section{Three carbonyl modes}

The $v(\mathrm{C}=0)$ curve cannot be fitted unless a third $\mathrm{C}=\mathrm{O}$ peak is introduced to match the low energy region of the $v(C=0)$ envelope. The fitted curves match the experimental curves very well using three $v(C=0)$ modes as shown in Figure 7. It is well known that the hydrogen bonding strengths the $v(\mathrm{C}=\mathrm{O})$ bond of the carboxylic acids and shifts the peak frequency of the $v(\mathrm{C}=\mathrm{O})$ mode to lower energy by $20 \mathrm{~cm}^{-1}$ to $80 \mathrm{~cm}^{-1}$ depending upon the strength of the hydrogen bonding, i.e. the stronger the bonding the larger the shift ${ }^{26-31}$.

There is a $v(C=0)$ mode at $1680 \mathrm{~cm}^{-1}$ in addition to the non-hydrogenbond $v(C=0)$ mode and the hydrogen-bond dimeric $v(\mathrm{C}=0)$ mode. The $v(\mathrm{C}=\mathrm{O})$ mode at $1680 \mathrm{~cm}^{-1}$ is assigned to the hydrogen-bonded polymeric $v(C=O)$ mode which has been discussed in detail elsewhere ${ }^{32}$. It is believe that the three structures are a function of the distance between the two most adjacent carboxylic acid neighbors, $d$. When $d>7.0 \AA, 7.0 \AA>d>5.0 \AA$, and $\mathrm{d}<5.0 \AA$, the majority carboxyl groups are the hydrogen-bonded dimeric $\mathrm{C}=\mathrm{O}$, the hydrogen-bonded polymeric $\mathrm{C}=\mathrm{O}$, and the non-hydrogen-bonded $\mathrm{C}=\mathrm{O}$, respectively.

For a MUA $/ \mathrm{Au}$, the peak frequencies of the non-hydrogen-bonded $v(\mathrm{C}=\mathrm{O})$ mode, the hydrogen-bonded dimeric $v(\mathrm{C}=\mathrm{O})$ mode and the hydrogenbonded polymeric $v(C=0)$ mode are at $1743 \mathrm{~cm}^{-1}, 1717 \mathrm{~cm}^{-1}, 1680 \mathrm{~cm}^{-1}$, respectively. The hydrogen-bonded dimeric $\mathrm{C}=\mathrm{O}$ is believed to have the sideto-side hydrogen bonding instead of a head-to-head hydrogen bonding ${ }^{4}$. 


\section{Esterification of MUA/Au monolayers}

\section{IRRAS}

Figures 9 and 10 show the IRRAS spectra in the high energy and the low energy region for a MUA/Au with fractional coverage of 0.50 prior to and after esterification, respectively. In Figure 9, there are very weak $\mathrm{CH}_{3}$ modes at $2965 \mathrm{~cm}^{-1}$ and $2875 \mathrm{~cm}^{-1}$ after $330 \mathrm{~min}$ of reaction time. This weak intensity is mainly a result of the small cross section found for related ester-terminated monolayers and their adsorbate precursors of $\mathrm{MUA}^{4}$. After $330 \mathrm{~min}$ of reaction time, the peak energies for asymmetric $v\left(\mathrm{CH}_{2}\right)$ mode and symmetric $v\left(\mathrm{CH}_{2}\right)$ mode shift to lower energy by $8 \mathrm{~cm}^{-1}$ and $6 \mathrm{~cm}^{-1}$, respectively. This indicates that the esterification process strengthen the polymethylene chainchain interaction from a liquid-like phase to a crystalline-like phase ${ }^{25}$. At the same time, the decrease in the peak area of these two modes after esterification suggests that this film has better ordering than the one before esterification.

Before esterification, MUA/Au has only one strong $v(C=0)$ peak at $1717 \mathrm{~cm}^{-1}$ which is the hydrogen-bonded dimeric $v(\mathrm{C}=0)$ mode of the acidterminated thiolate monolayer as shown in Figure 10. After esterification, MUA/Au has several strong peaks between $1000 \mathrm{~cm}^{-1}$ and $1400 \mathrm{~cm}^{-1}$ which are the fingerprint of the ester ${ }^{4}$ in addition to a different $v(C=0)$ mode. The peak at $1180 \mathrm{~cm}^{-1}$ and $1208 \mathrm{~cm}^{-1}$ are associated with the two newly formed $v(\mathrm{C}-\mathrm{O})$ modes $^{4}$. The hydrogen-bonded $v(\mathrm{C}=\mathrm{O})$ peak at $1717 \mathrm{~cm}^{-1}$ is converted to the non-hydrogen-bonded $v(\mathrm{C}=\mathrm{O})$ peak at $1743 \mathrm{~cm}^{-1}$ after esterification.

The conversion of the $v(\mathrm{C}=\mathrm{O})$ from a hydrogen-bonded mode to a nonhydrogen-bonded mode allows the extent of esterification to be monitored and calculated as shown in Figure 11.(see below) In fact, there is a relatively small hydrogen-bonded polymeric $v(C=0)$ peak at $1680 \mathrm{~cm}^{-1}$ in addition to these two strong $v(\mathrm{C}=\mathrm{O})$ peaks as shown in Figure 7. No obvious change in the hydrogen-bonded polymeric $v(\mathrm{C}=0)$ peak as a function of reaction time, as shown in Figure 12, indicates that the hydrogen-bonded polymeric $v(C=0)$ group behaves totally differently from the hydrogen-bonded dimeric $v(C=O)$ group due to the strong network-type hydrogen bond in terms of its reactivity. 

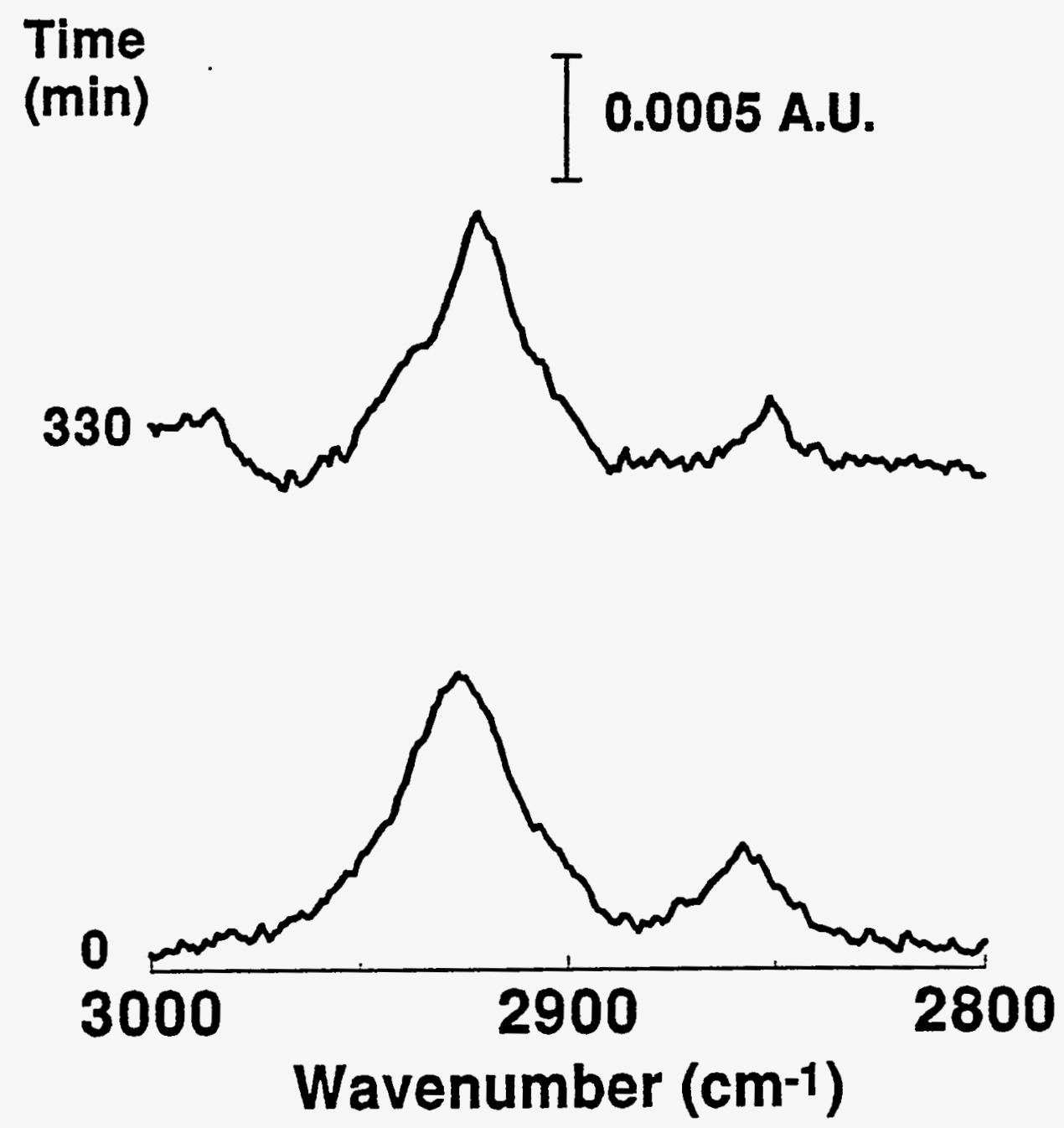

Figure 9. The IRRAS spectra in the high energy region of a half-coverage MUA/Au monolayer in $20 \%(\mathrm{v} / \mathrm{v}) \mathrm{HCl}$ ethanol solution for 0 and $330 \mathrm{~min}$. 


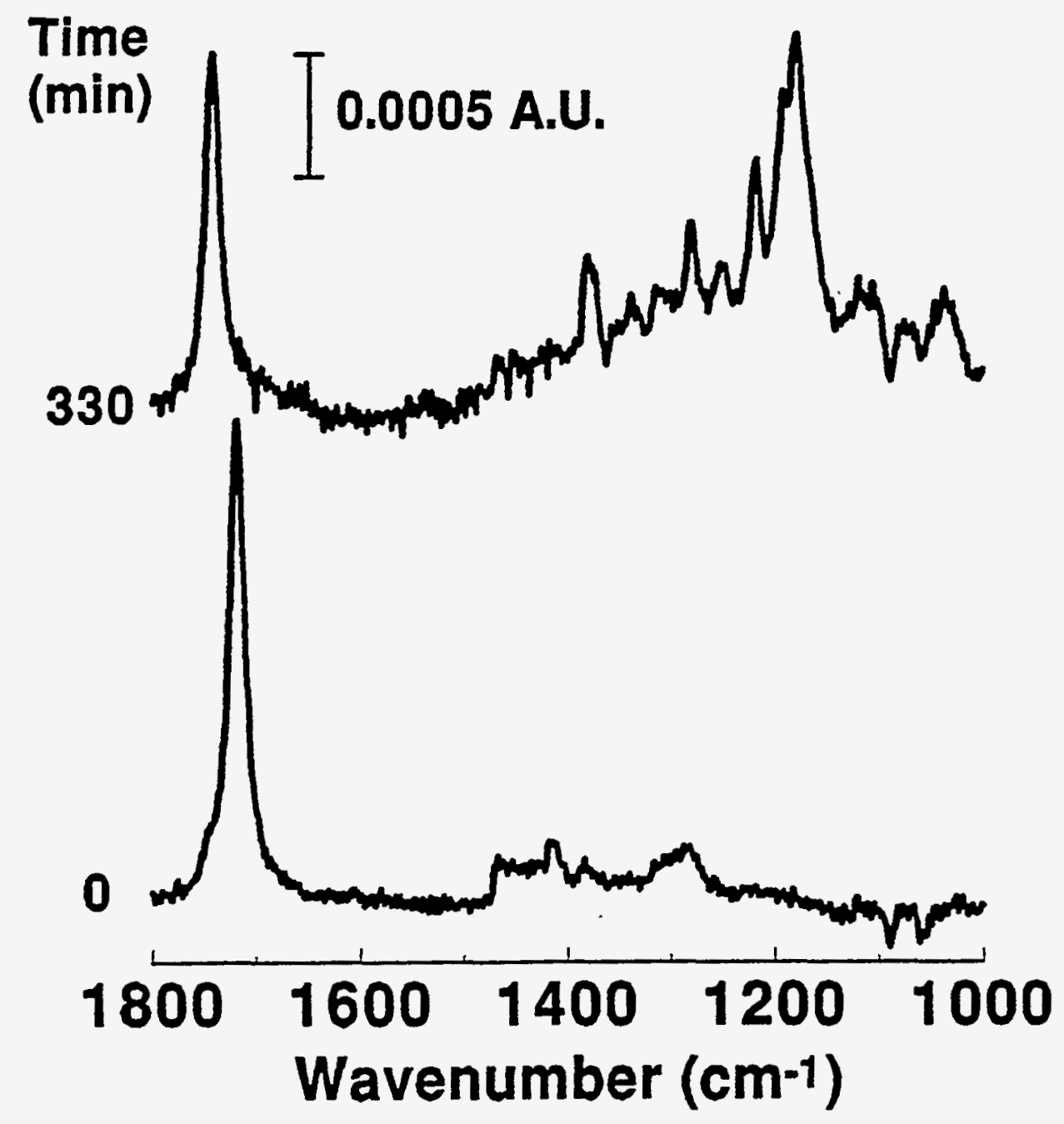

Figure 10. The IRRAS spectra in the low energy region of a half-coverage MUA/Au in $20 \%(\mathrm{v} / \mathrm{v}) \mathrm{HCl}$ ethanol solution for 0 and $330 \mathrm{~min}$. 


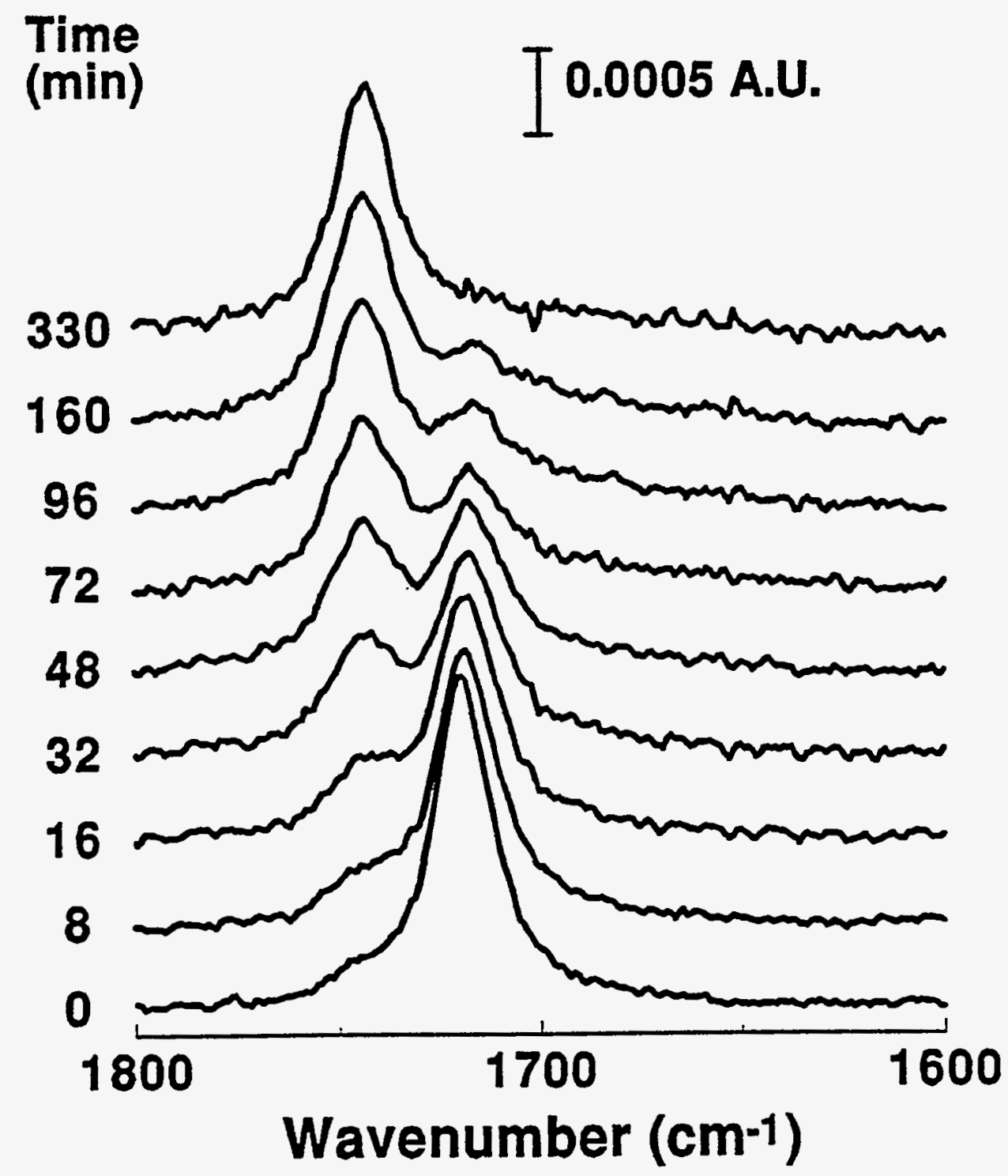

Figure 11. The IRRAS spectra in the $v(\mathrm{C}=\mathrm{O})$ region of a half-coverage MUA/Au monolayer as a function of immersion time in $20 \%$ (v/v) $\mathrm{HCl}$ ethanol solution. 


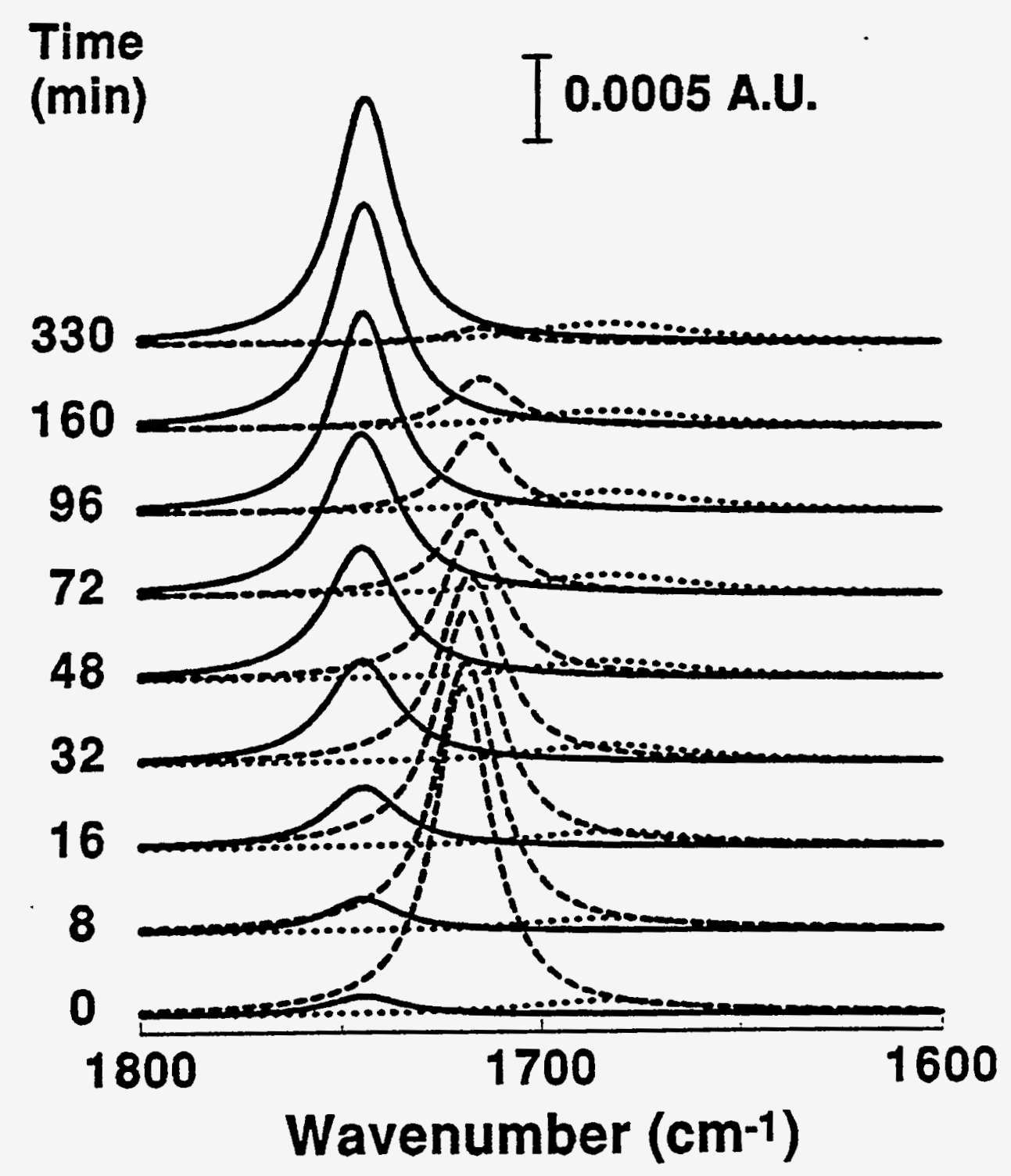

Figure 12. The deconvoluted $v(\mathrm{C}=\mathrm{O})$ modes of a half-coverage MUA/Au in $20 \%(\mathrm{v} / \mathrm{v}) \mathrm{HCl}$ ethanol solution as a function of immersion time. 
This result is consistent with the finding from the surface titration experiment discussed in Paper III of this dissertation. The kinetic data can be obtained from the deconvoluted peaks for the half-coverage monolayer as a function of reaction time, as shown in Figures 11-13, but not for the full-coverage monolayer because the IRRAS peak intensities of both the hydrogen-bonded dimeric $v(C=0)$ at $1717 \mathrm{~cm}^{-1}$ and the non-hydrogen-bonded $v(C=0)$ at 1743 $\mathrm{cm}^{-1}$ are weak for the full-coverage MUA/Au monolayer.

\section{Contact angle measurements}

As expect, the conversion of the hydrogen-bonded dimeric $v(\mathrm{C}=0)$ to the non-hydrogen-bonded $v(\mathrm{C}=\mathrm{O})$ due to esterification dramatically changes the wetting properties of the surface. This is shown in Figure 14. The contact angle, $\theta_{\mathrm{a}}$, increases from $25.0^{\circ}$ to $66.0^{\circ}$ and from $8.0^{\circ}$ to $66.0^{\circ}$ at the halfcoverage and full-coverage acid monolayers due to esterification, respectively. This strongly confirms that the higher surface energy acid monolayers are indeed transferred to a lower surface energy ester monolayer ${ }^{6}$ due to esterification. Like the IRRAS data, these data also provide an opportunity to evaluate the rate of esterification as a function of reaction time and the kinetic information. The next section examines the kinetics.

Kinetic analysis

IRRAS

The extent of esterification, $\theta$, of the half-coverage MUA/Au monolayer can be calculated from the IRRAS spectra with the following equations:

$$
\theta=\left(A_{a c i d}-A_{t a}\right) / A_{a c i d}
$$

where $A_{t a}$ is the peak area of the hydrogen-bonded dimeric $v(C=0)$ at 1717 $\mathrm{cm}^{-1}$ at a given time in the course of the reaction and $\mathrm{A}_{\mathrm{acid}}$ is the peak area before the onset of esterification, or

$$
\theta=A_{t e} / A_{\text {ester }}
$$




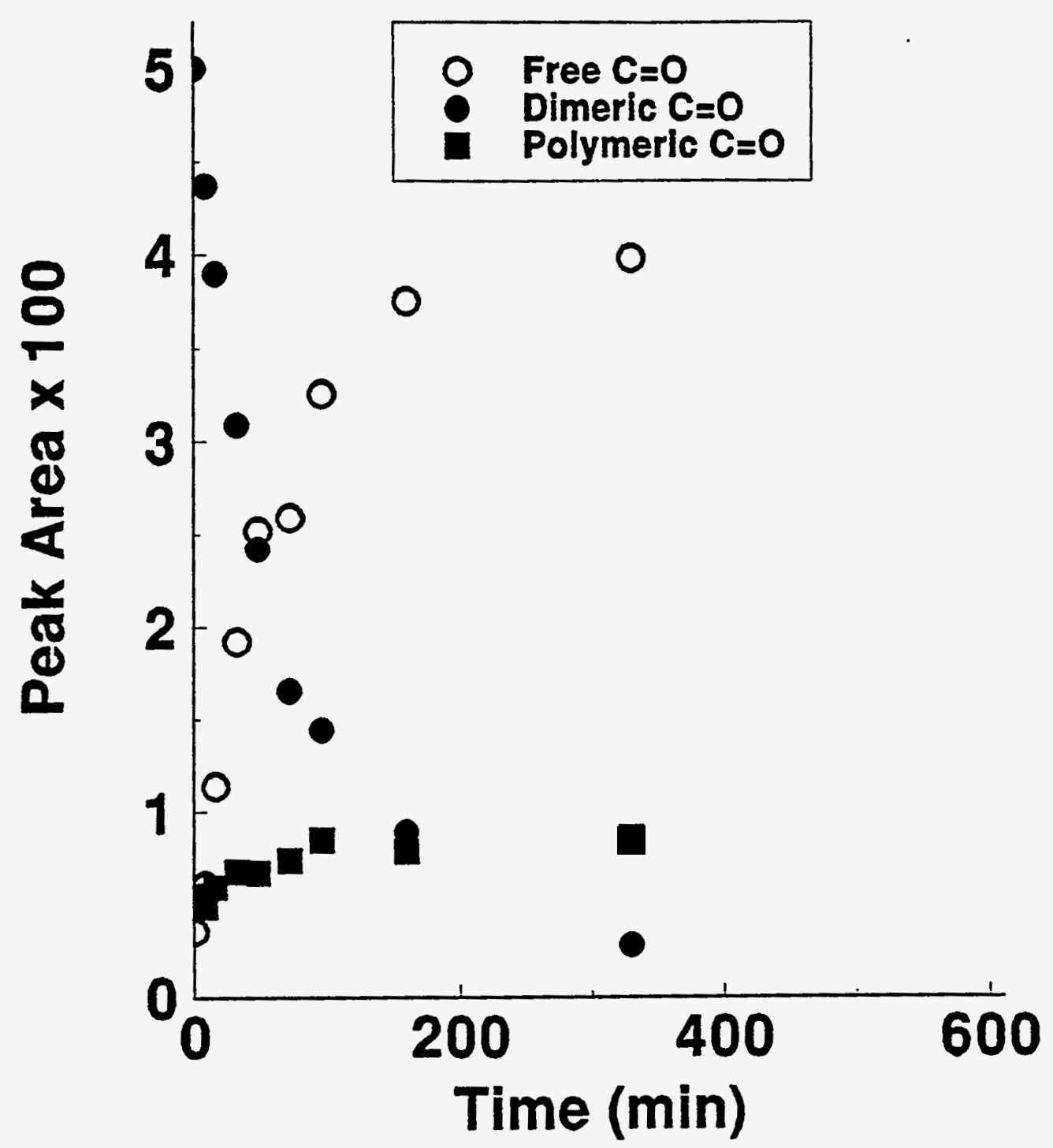

Figure 13. The peak area of the deconvoluted $v(C=0)$ modes of a halfcoverage MUA/Au $\AA$ as a function of immersion time in $20 \%$ ( $v / v) \mathrm{HCl}$ ethanol solution. 


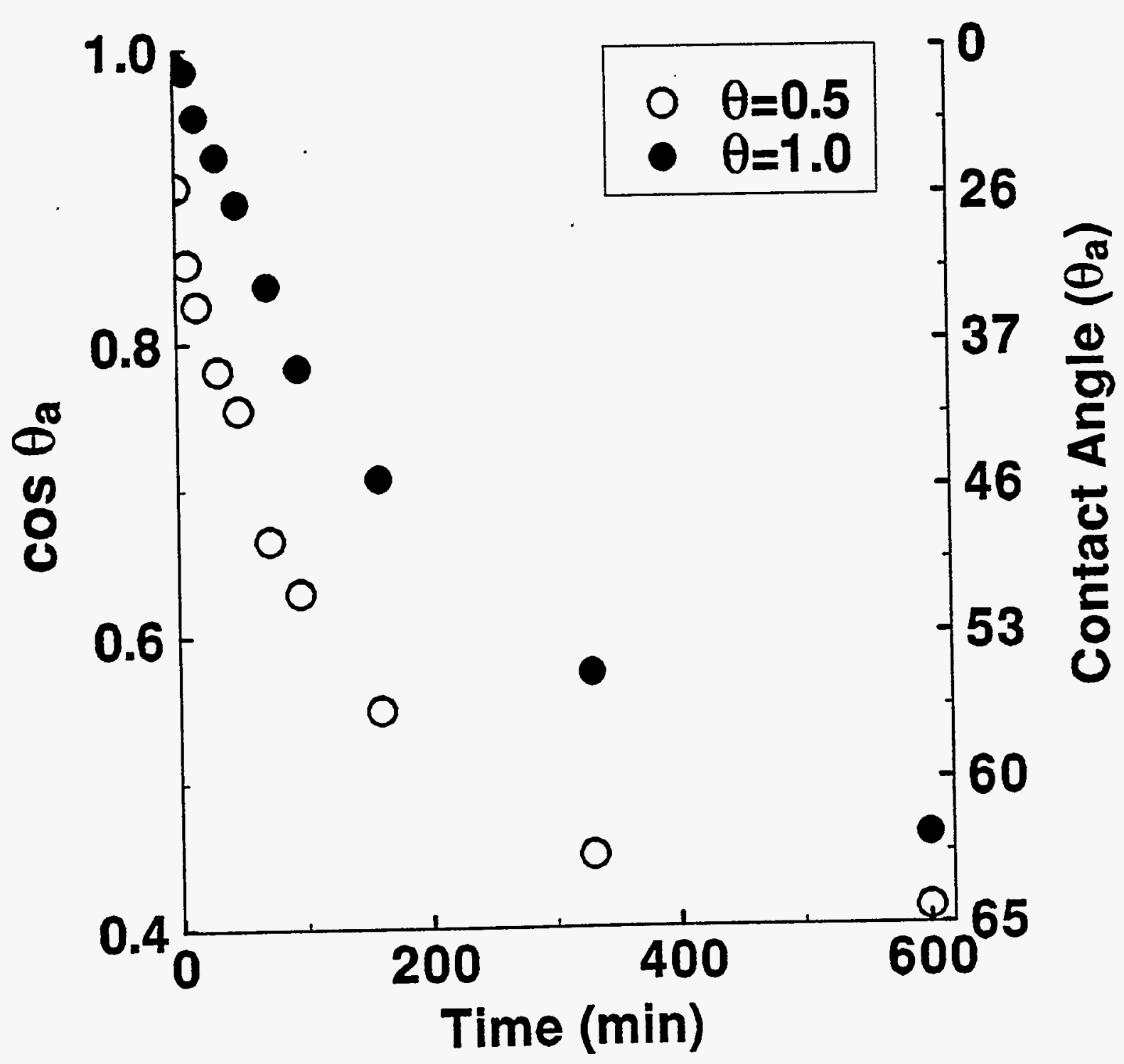

Figure 14. $\cos \theta_{\mathrm{a}}$ of $\mathrm{pH} 1.0 \mathrm{HCl}$ aqueous solution at a half-coverage MUA/Au monolayer and a full-coverage MUA/Au monolayer as a function of immersion time in $20 \%(\mathrm{v} / \mathrm{v}) \mathrm{HCl}$ ethanol. solution. 
where $A_{t e}$ is the peak area of the non-hydrogen-bonded $v(C=0)$ at 1743 $\mathrm{cm}^{-1}$ at a given time in the course of the reaction and $A_{\text {ester }}$ is the peak area after esterification is complete.

Equation 2 follows the process via reactant loss, and Equation 3 via product formation. Consistent results from both equations indicates that the conservation of the orientation of the monolayer is valid.

The linear relationship between the value of $\ln (1-\theta)$ and reaction time suggests that the surface esterification reaction is a first-order reaction as shown in Figure 15.

Unfortunately, these two equations were not applied for full-coverage MUAVAu monolayers because the IRRAS peak intensities of both the hydrogen-bonded dimeric $v(C=0)$ peak at $1717 \mathrm{~cm}^{-1}$ and the non-hydrogenbonded $v(C=O)$ peak at $1743 \mathrm{~cm}^{-1}$ are weak for full-coverage MUA/Au monolayers.

Contact angle measurements

The extent of esterification, $\theta$, at MUA/Au monolayers were calculated from contact angle measurement data by using the Cassie equation as follow:

$$
\cos \theta_{\mathrm{a}}=\theta \cos \theta_{\mathrm{a}, \mathrm{ester}}+(1-\theta) \cos \theta_{\mathrm{a}, \mathrm{acid}}
$$

where $\theta_{\mathrm{a}, \text { ester }}$ and $\theta_{\mathrm{a}, \text { acid }}$ are the contact angles at the $100 \%$ ester monolayer and the $100 \%$ acid monolayer, respectively.

The linear relationship between the $\ln (1-\theta)$ and reaction time from both IRRAS and contact angle measurement data concludes this surface esterification is a first-order reaction as shown in Figure 15. The contact angle measurement data have better reproducibility than the IRRAS data simply because the contact angle measurement data are the average of four repeat measurements while the IRRAS data are from a single measurement. The values of the error bar for the contact angle measurements and IRRAS are $1^{\circ}$ and $5 \times 10^{-5}$ A.U., respective. Since both techniques give the same result for the half-coverage MUA/Au, both can be used to measured the rate for the fullcoverage MUA/Au monolayer as well. Only the contact angle 


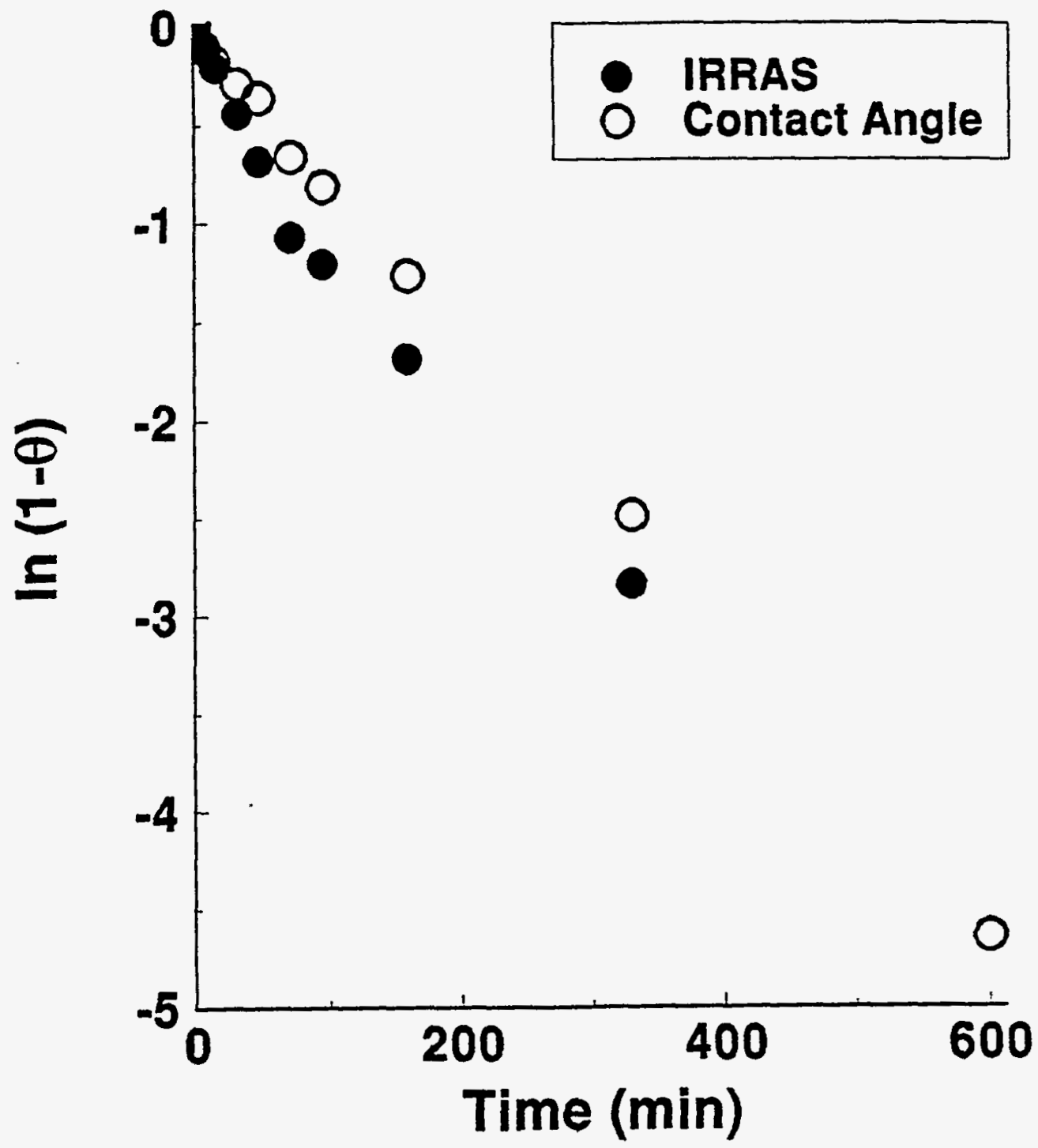

Figure 15. $\ln (1-\theta)$ from IRRAS and contact angle measurement data for a half-coverage MUA/Au monolayers in $20 \%(\mathrm{v} / \mathrm{v}) \mathrm{HCl}$ ethanol solution as a function of immersion time. 
measurements technique was used rather the IRRAS because the IRRAS peak intensities of both the hydrogen-bonded dimeric $v(C=0)$ peak and the nonhydrogen-bonded $v(C=O)$ peak are weak for full-coverage MUA/Au monolayers.

Since ethanol was used as the solvent, this first-order reaction most likely is a pseudo first-order reaction.

For a first-order reaction, the rate constants are equal to the slope of the $\ln (1-$ . $\theta$ ) vs, reaction time as shown in Figure 16 and were calculated to be $7.62 \mathrm{x}$ $10^{-3} \mathrm{sec}^{-1}$ and $3.87 \times 10^{-3} \mathrm{sec}^{-1}$ for a packing density of $4.0 \times 10^{-10} \mathrm{~mol} / \mathrm{cm}^{2}$ and $8.0 \times 10^{-10} \mathrm{~mol} / \mathrm{cm}^{2} \mathrm{MUA} / \mathrm{Au}$ monolayers, respectively. This indicates that the rate constant is a reciprocal function of the packing density of the thiolate monolayer. 


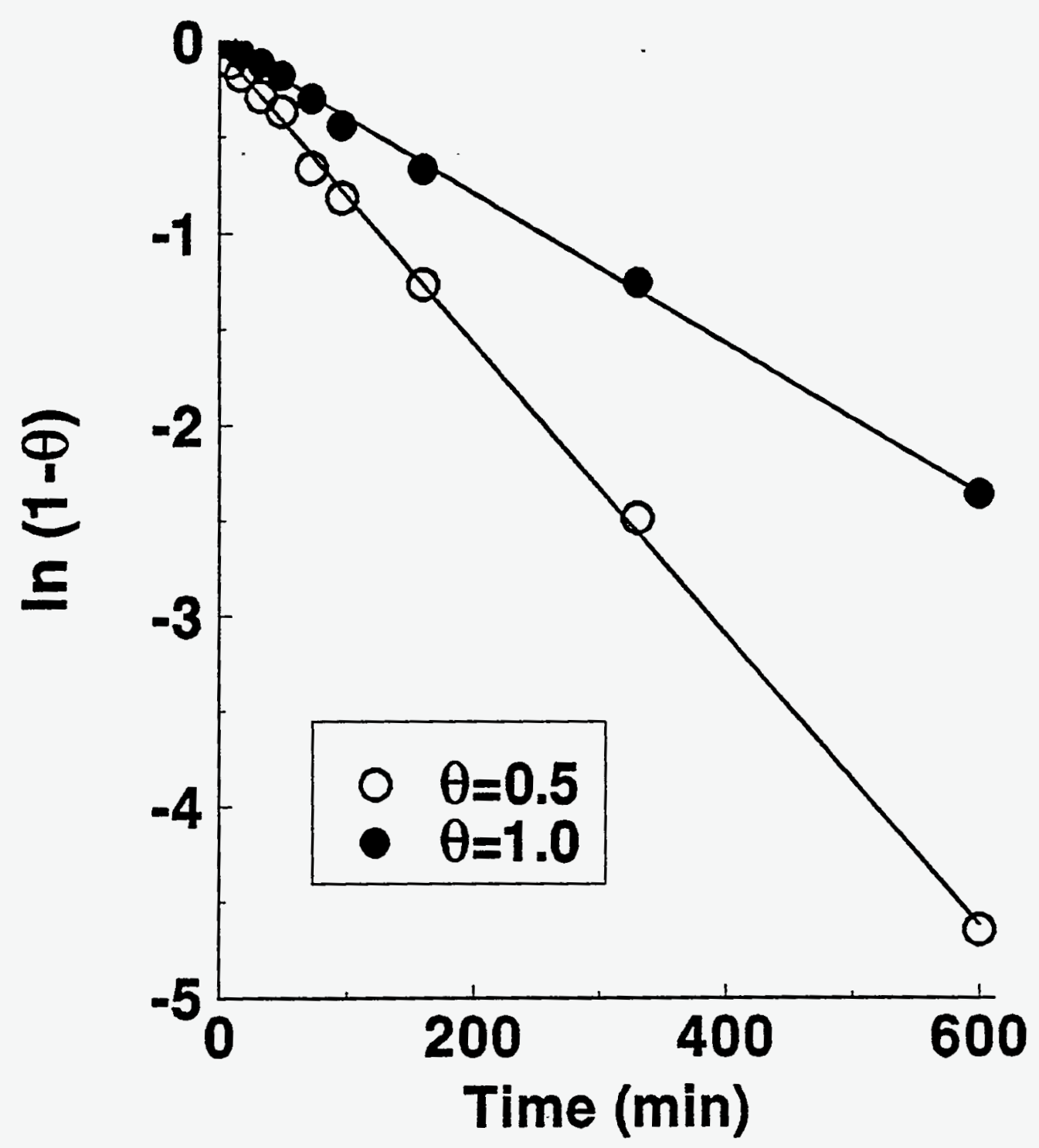

Figure 16. $\ln (1-\theta)$ of a half-coverage MUA/Au monolayer and a fullcoverage MUAAu monolayer as a function of immersion time in $20 \%(\mathrm{v} / \mathrm{v}) \mathrm{HCl}$ ethanol solution. 


\section{8}

\section{CONCLUSIONS}

The adsorption process of the MUA on $\mathrm{Au}$ is at least a two-step process with a very fast reaction rate for the first step followed by a very slow reaction rate for the second step.

Esterification transforms the hydrogen-bonded dimeric $v(C=0)$ peak at $1717 \mathrm{~cm}^{-1}$ completely to the non-hydrogen-bonded $v(C=0)$ peak at $1743 \mathrm{~cm}^{-1}$ without change the hydrogen-bonded polymeric $v(C=O)$ group due to the strong network-type hydrogen bond of the hydrogen-bonded polymeric $v(C=0)$ group. Esterification process stabilizes the polymethylene chain from liquid-like phase to crystalline-like phase.

Surface reaction can be monitored with the IRRAS or the contact angle measurement. This surface esterification is a pseudo-first-order reaction with a rate constant of $7.62 \times 10^{-3} \mathrm{sec}^{-1}$ and $3.87 \times 10^{-3} \mathrm{sec}^{-1}$ for a packing density of $4.0 \times 10^{-10} \mathrm{~mol} / \mathrm{cm}^{2}$ and $8.0 \times 10^{-10} \mathrm{~mol} / \mathrm{cm}^{2}$ MUA $/$ Au monolayers, respectively. The rate constant of surface esterification is a reciprocal function of the packing density of the thiolate monolayer. 


\section{REFERENCES}

(1) Bain, C. D.; Whitesides; G. M. Angew. Chem., Int. Ed. Engl. 1989, 28, 506 and references therein.

(2) Whitesides, G. M.; Laibinis, P. E. Langmuir 1990, 6, 87 and references therein.

(3) Ulman, A An Introduction to Ultra-Thin Organic Films From Langmuir-Blodgett to Self-Assembly; Academic Press: San Diego, 1991.

(4) Nuzzo, R. G.; Dubois, L. H.; Allara, D. L. J. Am. Chem. Soc. 1990, $112,558$.

(5) Snyder, R. G.; Schachtschneider, J. H. Spectrochim. Acta 1963, $19,85$.

(6) Holmes-Farley, S. R.; Whitesides, G. M. Langmuir 1987, 3, 60.

(7) Widrig, C. A.; Alves, C. A.; Porter, M. D. J. Am. Chem. Soc. 1991, $113,2805$.

(8) Widrig, C. A.; Chung, C.; Porter, M. D. J. Electroanal. Chem. 1991, 310,335 .

(9) Azzam, G. A.; Bashara, N. M. Ellipsometry and Polarized Light; NorthHolland Publishing: Amsterdam, 1977.

(10) Handbook of Chemistry and Physics; Weast, R. C., Ed.; Chemical Rubber Cor.: Boca Raton, FL, 1981.

(11) Porter, M. D.; Bright, T. B.; Allara, D. L.; Chidsey, C. E. D. J. Am. Chem. Soc. 1987, 109, 3559.

(12) Finklea, H. O.; Avery, S.; Lynch, M.; Furtsch, T. Langmuir 1987, 3, 409.

(13) Troughton, E. B.; Bain, C. D.; Whitesides, G. M.; Nuzzo, R. G.; Allara, D. L.; Porter, M. D. Langmuir 1988, 4, 365.

(14) Tao, Y.-T.; Lee, M.-T.; Chang, S.-C. J. Am. Chem. Soc. 1993, 115, 9547.

(15) Bain, C. D.; Whitesides, G. M. J. Am. Chem. Soc. 1989, 111, 7164.

(16) Ulman, A. J. Mater, Educ. 1989, 11, 205.

(17) Stole, S. M.; Porter, M. D. Appl. Spectrosc. 1990, 44, 1418.

(18) Walczak, M. M.; Chung, C.; Stole, S. M.; Widrig, C. A.; Porter, M. D. J. Am. Chem. Soc. 1991, 113, 2370.

(19) Wilson, E. B., Jr.; Decius, J. C.; Cross, P. C. Molecular Vibrations; McGraw-Hill: New York, 1955, pp 285. 
(20) Bain, C. D.; Troughton, E. B.; Tao, Y-T.; Evall, J.; Wihitesides, G. M.; Nuzzo, R. G. J. Am. Chem. Soc. 1989, 111, 321.

(21) Shimazu, K.; Yagi, I.; Uosaki, K. Langmuir 1992, 8, 1385.

(22) Buck, M.; Eisert, F.; Fischer, J. Grunze, M.; Träger, F. Appl. Phys. 1991, A53, 552.

(23) Buck, M.; Eisert, F.; Fischer, J. Grunze, M.; Träger, F. J. Vac. Sci. Technol. 1992, A10, 926.

(24) Thomas, R. C.; Sun, L.; Crooks, R. M.; Ricco, A. J. Langmuir 1991, 7, 620.

(25) Snyder, R. G.; Strauss, H. L.; Elliger, C. A. J. Phys. Chem. 1982, 86, 5145.

(26) Gramstad, T.; Fuglevik, J. Spectrochim. Acta 1965, 21, 343.

(27) Pullin, J. A.; Werner, J. Spectrochim. Acta 1965, 21, 1257.

(28) Figeys, H. P.; Nasielski, J. Spectrochim. Acta 1967, 23A, 465.

(29) Bellamy, L. J.; Rogash, P. E. Spectrochim. Acta 1960, 16, 30.

(30) Collins, A. J.; Morgan, K. J. J. Chem. Soc. 1963, 3437.

(31) Brooks, C. J. W. J. Chem. Soc. 1960, 661.

(32) Paper III of this dissertation. 


\section{GENERAL SUMMARY AND PROSPECTS}

Deprotonation causes the heterogeneous electron transfer rate of ferricyanide at phenylcarboxylic acids and pyridylcarboxylic acids coated Pt electrodes to decrease by $0 \%$ to $40 \%$ and $85 \%$, respectively, and decreases the potential at reaction plane of the phenylcarboxylic acid and the pyridylcarboxylic acid monolayers on platinum by 0 to $5 \mathrm{mV}$ and $\sim 20$ $\mathrm{mV}$, respectively. The observed $\Delta \phi_{\mathrm{r}}$ values due to deprotonation for phenylpropanoic acid/Pt and phenylbutanoic acid/Pt are only $0.7 \%$ of the theoretical values and that for isonicotinic acid/Pt and nicotinic acid/Pt are only $1.2 \%$. No change in $\phi_{\mathrm{r}}$ was observed for benzoic acid/Pt and phenylacetic acid/Pt electrodes. Pyridylcarboxylic acids becomes weaker acids upon immobilization onto a Pt surface primarily due to hydrogen bonding.

At very low coverage, all the polymethylene chains of thiolate monolayers lay on gold and form the $\mathrm{C}-\mathrm{H}$...Au agostic bond at $2903 \mathrm{~cm}^{-1}$ with $v_{\mathrm{a}}\left(\mathrm{CH}_{2}\right)$ and $v_{\mathrm{s}}\left(\mathrm{CH}_{2}\right)$ dipole perpendicular and parallel to the $\mathrm{Au}$ surface, respectively. At increasing coverage, the polymethylene chain must turn the chain axis in order to stand up to accommodate more thiol molecules to be adsorbed on Au surface so that the amount of the agostic $v_{\mathrm{a}}\left(\mathrm{CH}_{2}\right)$ decreases and disappears at high coverage.

The hydrogen-bonded dimeric $\mathrm{C}=\mathrm{O}$ is the dominated $\mathrm{C}=\mathrm{O}$ peak of carboxylic acid-terminate thiloate monolayers on $\mathrm{Au}$ at low coverage. With increasing coverage, the amount of the hydrogen-bonded dimeric $\mathrm{C}=\mathrm{O}$ decreases while the amount of both the non-hydrogen-bonded and the hydrogen-bonded polymeric $C=O$ groups increase, meanwhile the surface wetting property increases remarkably with increasing coverage.

The $\mathrm{pK}_{\mathrm{a}}$ and the titration transition range of the non-hydrogenbonded $\mathrm{C}=\mathrm{O}$, the hydrogen-bonded dimeric $\mathrm{C}=\mathrm{O}$, and the hydrogen- 
bonded polymeric $\mathrm{C}=\mathrm{O}$ groups of carboxylic acid-terminate thiloate monolayers on $\mathrm{Au}$ are determined from IRRAS data to be $\sim 6, \sim 8$, and $\sim 10$, and 3,7 , and $3 \mathrm{pH}$ units, respectively. The effective $\mathrm{pK}_{\mathrm{a}}$ of the nonhydrogen-bonded $\mathrm{C}=\mathrm{O}$ and the hydrogen-bonded dimeric $\mathrm{C}=\mathrm{O}$ groups increases from 5 up to $\sim 10$ with increasing the extent of deprotonation.

Esterification of the 11-mercaptoundecanoic acid (MUA) monolayer on $\mathrm{Au}$ converts the $v(\mathrm{C}=\mathrm{O})$ at $1717 \mathrm{~cm}^{-1}$ of the carboxylic acid to the $v(C=O)$ at $1743 \mathrm{~cm}^{-1}$ of ester without change the hydrogen-bonded polymeric $v(\mathrm{C}=\mathrm{O})$ at $1680 \mathrm{~cm}^{-1}$ at all, meanwhile the contact angle at this monolayer increases from $\sim 10^{\circ}$ to $62^{\circ}$. Both IRRAS and contact angle measurements can be used to measure the reaction rate of this esterification process. The rate constant is proportional reciprocally to the packing density of the monolayer. For example the rate constants are $7.62 \times 10^{-3} \mathrm{sec}^{-1}$ and $3.87 \times 10^{-3} \mathrm{sec}^{-1}$ for a packing density of $4.0 \times 10$ ${ }^{-10} \mathrm{~mol} / \mathrm{cm}^{2}$ and $8.0 \times 10^{-10} \mathrm{~mol} / \mathrm{cm}^{2} \mathrm{MUA} / \mathrm{Au}$ monolayers, respectively.

The use of ion-pair electrolytes might be able to increase the change in the potential at the reaction plane due to less screening effect than conventional electrolytes. The use of different metal surfaces should form agostic bonds also with methylene groups. The reaction rate of different surface reactions can be measured in the same fashion using IRRAS and/or contact angle measurements. 


\section{ACKNOWLEDGMENTS}

It has been a pleasure to work with my major professor Dr. Marc D. Porter for the last eight years. He has been very helpful and very kind in so many things that I can not remember all. Special thanks I would like to give to Dr. Shelley J. Coldiron for setting up and maintaining the Dr. Porter group computer network system which goes crazy once in a while. I would very much like to thank Neal J. Simmsons not only for helping me in many thing in the lab but also teaching American slang and jokes. I particularly like to thank my program of study committee members including Dr. Dennis C. Johnson, Dr. Edward S. Yeung, Dr. Kai-Min Ho and Dr. Robert. A. Jacobson for their help and support. Finally, I would like to thank all the people who have helped me in this study. They are En-Yi Ting, Dr. Chuan-Jian Zhong, Dr. Jerzy Zak, Dr. Mark McDermott, Dr. Christie McDermott, Dr. Scott M. Stole, Dr. Thomas P. Jones, Man Kit Ho, Brain Lamp, Jame W. Anderegg, Dr. Cindra A. Widrig, Dr. Mary M. Walczak, Dr. Lia-Kwan Chau, Bikas Vaidya, Randy S. Deinhammer, Marilyn Forsling, John B. Green, Mohamed I. Omer and those Ames Lab employees who have helped me. I particularly enjoy the annual volleyball game between electrochemistry group I and II. Who will be the winner this summer?

This work was performed at Ames Laboratory under Contract No. W-7405-Eng-82 with the U.S. Department of Energy. The United States government has assigned the DOE Report number IS-T 1671 to this thesis. 